\title{
DUAL-ENERGY CT AND CT PERFUSION FOR IMPROVED CT STROKE IMAGING
}

FASCO VAN OMMEN

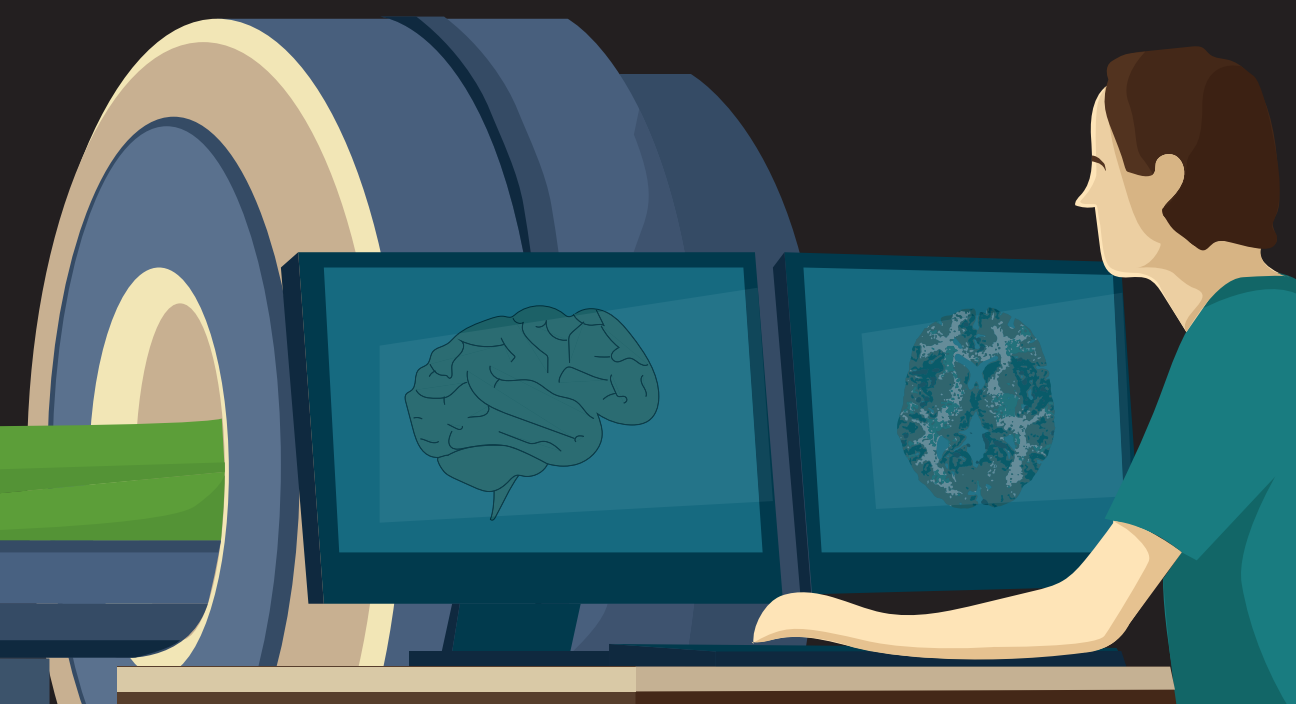





\section{DUAL-ENERGY CT AND CT PERFUSION FOR IMPROVED CT STROKE IMAGING}

FASCO VAN OMMEN 


\section{Dual-energy CT and CT perfusion for improved CT stroke imaging}

Fasco van Ommen

PhD Thesis, University Utrecht, the Netherlands

ISBN 978-90-393-7336-1

Layout and cover design by Elisa Calamita, www.persoonlijkproefschrift.nl Printing: Ridderprint | www.ridderprint.n|

All rights reserved. No part of this thesis may be reproduced, stored or transmitted in any way or by any means without the prior permission of the author. The copyright of the articles that have been published has been transferred to the respective journals.

(c) Fasco van Ommen, 2020 


\title{
Dual-energy CT and CT perfusion for improved CT stroke imaging
}

\author{
Duale-energie CT en CT perfusie voor verbeterde \\ CT beeldvorming bij beroertes \\ (met een samenvatting in het Nederlands)
}

\begin{abstract}
Proefschrift
ter verkrijging van de graad van doctor aan de

Universiteit Utrecht

op gezag van de

rector magnificus, prof.dr. H.R.B.M. Kummeling,

ingevolge het besluit van het college voor promoties

in het openbaar te verdedigen op
\end{abstract}

donderdag 26 november 2020 des ochtends te 9.15 uur

door

\section{Fasco van Ommen}

geboren op 18 juli 1992

te Hilversum 


\section{Promotoren:}

Prof. dr. ir. M.A. Viergever

Prof. dr. ir. H.W.A.M. de Jong

\section{Copromotoren:}

Dr. J.W. Dankbaar

Dr. ir. H.E. Bennink

The research described in this thesis is part of the research programme earlier recognition of cardiovascular diseases, which is financed by the Dutch Research Council (NWO; project number 14732) and the Dutch Heart Foundation (2015B031).

Financial support by the Dutch Research Council (NWO) and Dutch Heart Foundation for the publication of this thesis is gratefully acknowledged. 


\section{Contents}

1. Introduction 7

PART I: TECHNICAL ASPECTS OF DUAL-ENERGY CT 17

2. Radiation dose of a dual-layer CT scanner 19

3. Image quality of conventional CT images on a dual-layer CT scanner 33

4. Image quality of virtual monochromatic images in non-contrast brain CT 57

PART II: DUAL-ENERGY CT IN STROKE IMAGING

5. Virtual monochromatic non-contrast brain CT for detection of stroke 73

6. Virtual ischemia maps for core infarct volume estimation 91

$\begin{array}{ll}\text { 7. Virtual monoenergetic CT perfusion } & 109\end{array}$

PART III: CT PERFUSION IN STROKE IMAGING

8. Effects of acquisition interval on CT perfusion analysis 127

9. Thin-slice CT perfusion for detection of small-volume infarction 141

10. Summary and Discussion 161

11. Bibliography 175

$\begin{array}{lr}\text { APPENDICES } & 189\end{array}$

$\begin{array}{ll}\text { A. Nederlandse samenvatting } & 190\end{array}$

B. Acknowledgments 194

$\begin{array}{ll}\text { C. List of Publications } & 198\end{array}$

D. About the Author 200 



\section{CHAPTER 1}

INTRODUCTION

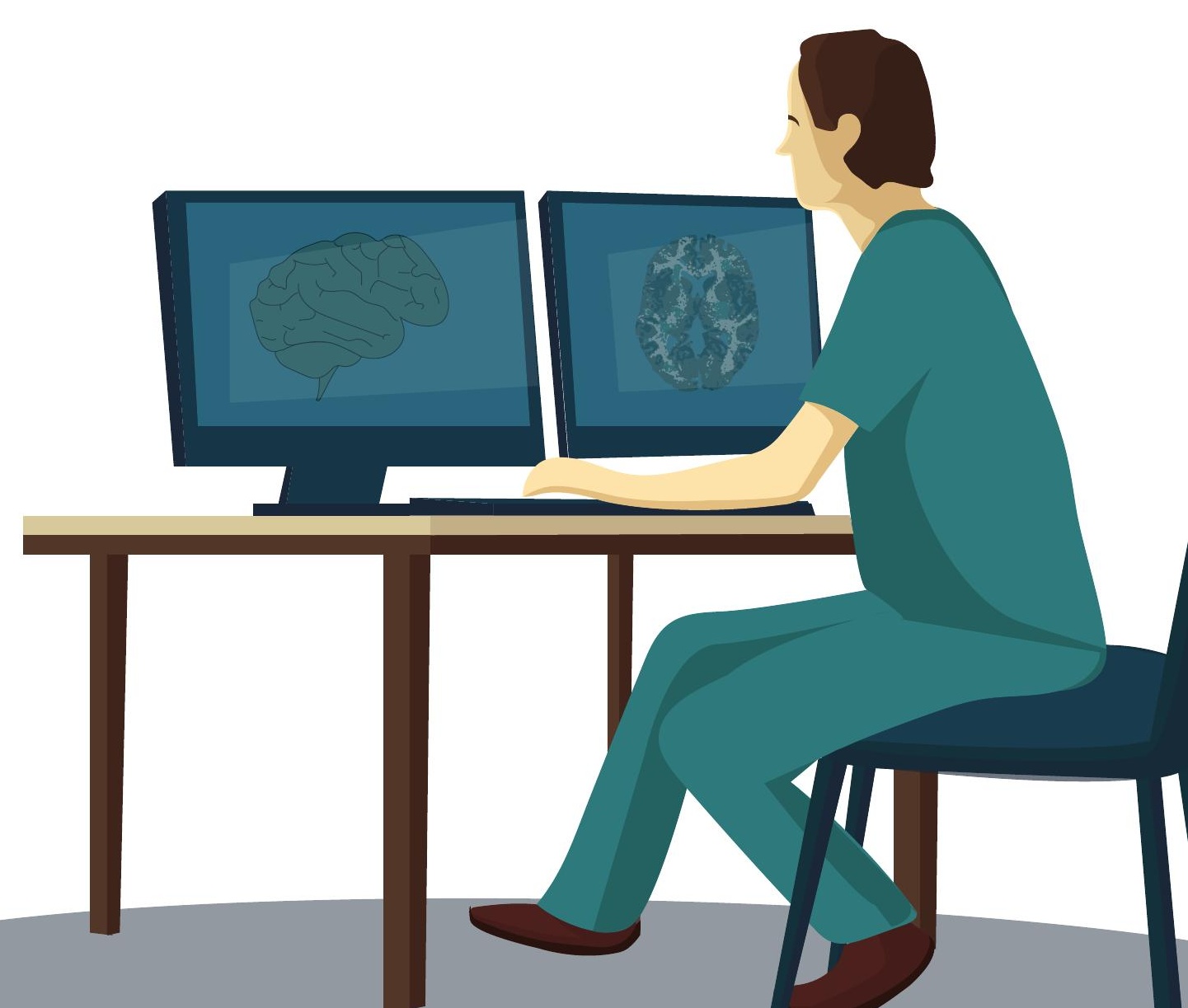




\section{Ischemic stroke}

Cardiovascular diseases (CVDs) are the leading causes of death in the world. An estimated 17.9 million people died from a CVD worldwide in 2016. The majority, 85\% of deaths, is caused by either a heart attack or a stroke[1]. In the Netherlands, 38199 people died of a CVD in 2018, of which 9176 died of a stroke[2]. Not only does stroke significantly contribute to mortality, it is also a leading cause of disability[3]. Because of the aging population, the number of stroke patients is expected to increase[4].

Strokes can be divided into two groups: 1) ischemic strokes caused by an arterial occlusion, for example by a (temporary) clot, and 2) hemorrhagic strokes caused by a rupture of a vessel[5]. In this thesis we will be focusing on ischemic strokes.

Currently, treatment options for ischemic strokes are intravenous administration of tissue plasminogen activator (tPA) for clot dissolution, intra-arterial thrombolysis, and endovascular mechanical clot removal[6]. Therapeutic interventions are most effective when initiated in the early stages after a stroke[7-9]. Guidelines state that intravenous thrombolysis should only be administered to patients within 4.5 hours, and endovascular treatment should only be initiated within 6 hours of ischemic stroke symptom onset[10]. Brain tissue damage may be irreversible, and the loss of brain tissue continues while the arterial occlusion persists. Therefore in stroke care the phrase "time is brain" is often used, meaning that the diagnosis and treatment of a stroke should be as fast as possible. This explains why the preferred imaging modality for acute stroke imaging usually is computed tomography (CT)[11]. CT is fast, relatively cheap and widely available. The acute stroke imaging protocol usually consists of noncontrast CT, CT perfusion and CT angiography scans.

\section{CT imaging in stroke}

\section{Non-contrast CT}

In stroke imaging, the first acquisition is non-contrast CT (NCCT) of the entire brain. The primary goal of NCCT is to differentiate between ischemic and hemorrhagic strokes. Since blood has a higher attenuation than brain tissue, it appears hyperdense on a CT scan and can therefore be easily detected (Figure 1.1A). In addition to differentiating ischemic from hemorrhagic strokes, NCCT also provides some information on the extent of cerebral ischemia. On NCCT, subtle loss of gray-white matter differentiation and evidence of swelling can be first indications of ischemic stroke[12]. The ischemic area appears hypodense on NCCT (Figure 1.1B). 


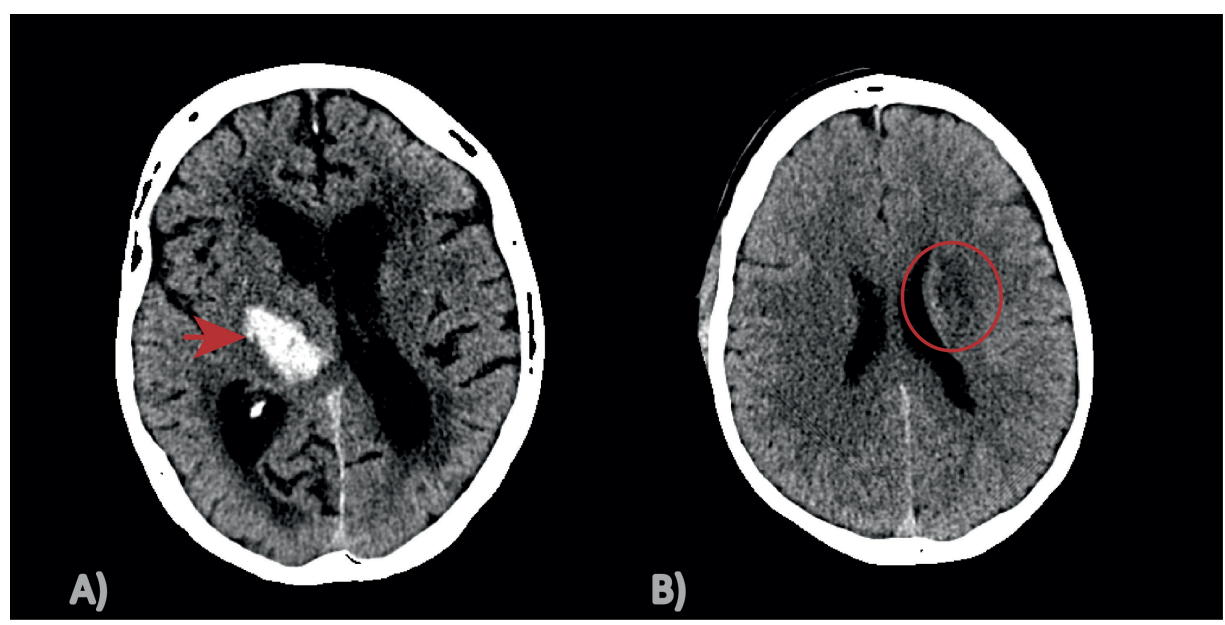

Figure 1.1 | Examples of A) intracranial hemorrhage (hyperdense area) on non-contrast CT and B) cerebral ischemia (hypodense area) on non-contrast CT.

\section{CT perfusion}

A CT perfusion (CTP) scan is a dynamic contrast-enhanced acquisition. A contrast bolus of iodinated contrast agent is injected into a peripheral vein and with a certain time interval the pertinent part of the brain is imaged for typically about a minute. In this way it becomes possible to monitor the wash-in and wash-out of contrast agent in the brain parenchyma (Figure 1.2) and to estimate brain perfusion parameters such as blood volume and flow. These parameters are indicative of the delivery of blood to the brain and thereby show whether brain tissue is already lost (infarct core), is still salvageable (infarct penumbra) or is unaffected (healthy) by the occlusion. Infarct penumbra is tissue that is at risk of becoming infarct core if recanalization of the artery is not achieved, i.e. the occlusion needs to be removed for the tissue at risk (penumbra) to be salvaged. In stroke treatment, the ratio between the size of infarct core and the infarct penumbra (mismatch volume) can be used to determine the eligibility of a patient for thrombolysis[13] or thrombectomy[14].

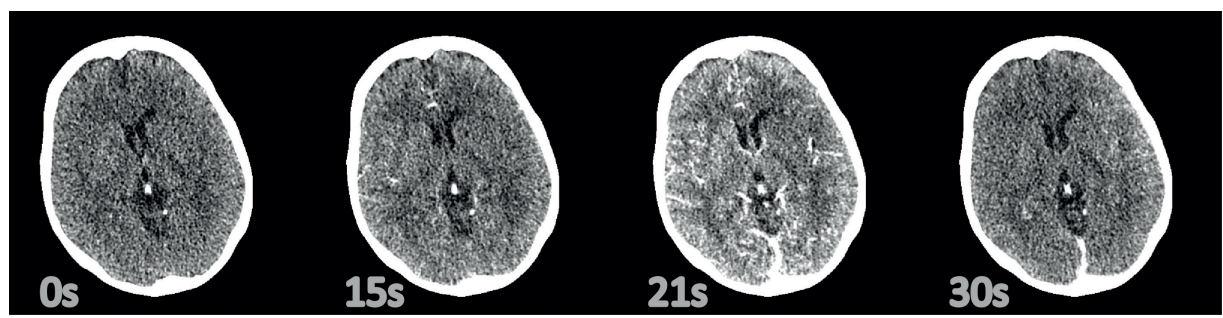

Figure 1.2 | Four acquisitions of a CT perfusion series. At 0 seconds there is no contrast enhancement visible yet. After 15 seconds the contrast enhances the large arteries, and at 21 seconds the enhancement is at its maximum. After 30 seconds the veins are still clearly enhanced. 


\section{CTP analysis}

To quantitatively analyze the CTP acquisition series, a number of pre-processing steps are required. First, head movement of the patient between time frames needs to be corrected by aligning the brain using automatic rigid image registration on the skull. Second, noise in the CTP scan (Figure 1.2) needs to be reduced. Noise reduction is typically achieved by applying an isotropic Gaussian filter or an edge-preserving filter. Following these pre-processing steps, a mathematical approach (perfusion modeling) is used to estimate perfusion parameters.

The passage of the contrast bolus is tracked by measuring the attenuation changes over time in every single voxel, the so called time-attenuation curves (TACS). We define tissue perfusion as a modeling system; a system with an input and a response. In CTP, the input of the system is the TAC of a large artery, which is called the arterial input function (AIF). To correct the input function of the system for partial volume effects, the amplitude of the AIF is scaled with the venous output function (VOF) (Figure 1.3A). The partial volume effect is calculated as the scaling between the area under the curve (AUC) of the AIF and the AUC of the VOF. The response of the system is defined as the TAC of the tissue of interest, e.g. gray or white matter (Figure 1.3B), which is expressed as the convolution of the partial volume corrected AIF with the impulse response function (IRF) of the system:

$$
T A C(t)=I R F(t) * A I F(t)
$$

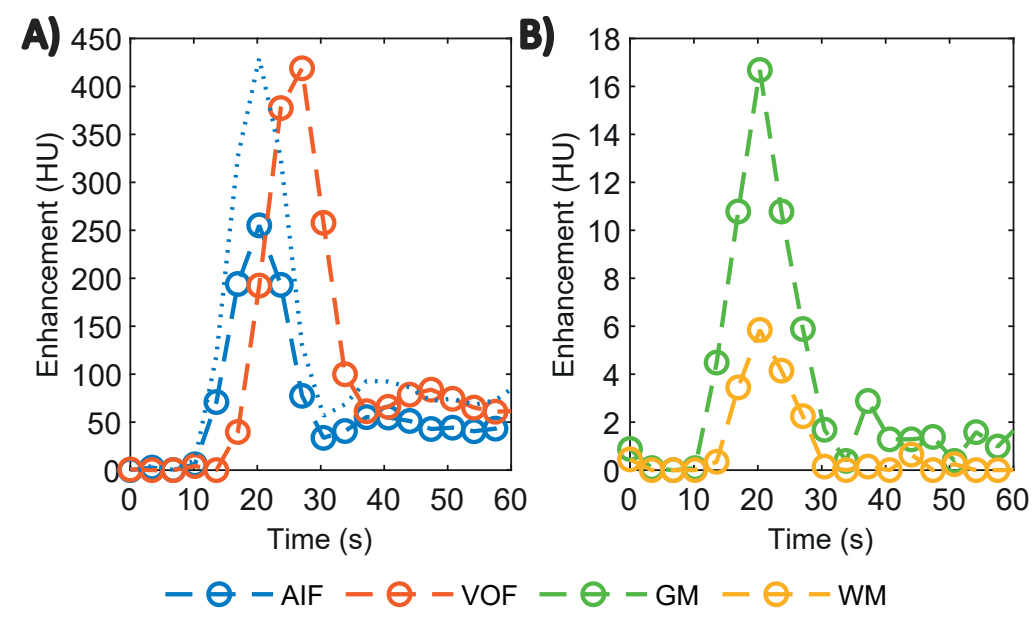

Figure 1.3 | Examples of contrast enhancement curves: A) arterial input function (AIF) and venous output function (VOF), B) gray matter (GM) and white matter (WM). The dashed line shows the partial volume corrected AIF. 
Since the $T A C(t)$ and the $A I F(t)$ are known, obtaining the $\operatorname{IRF}(t)$ is a so-called inverse problem. Multiple methods exist for estimating the IRF, amongst which (block-circulant) singular value decomposition ((b)SVD)[15,16] and non-linear regression[17]. Other methods such as the maximum slope method[18] try to directly estimate perfusion values from $T A C(t)$ and $A I F(t)$ without involving the $\operatorname{IRF}(t)$. These methods, however, often show biased measurements.

Solving the IRF is important, because it allows direct calculation of the tissue perfusion properties of the system. The tissue properties that are of importance to stroke imaging are those of the cerebral blood flow (CBF), cerebral blood volume (CBV) and mean transit time (MTT) (Figure 1.4). The height of the IRF is equal to the blood flow and the area under the curve of the IRF is equal to the blood volume. The MTT is interrelated to the CBF and CBV by the so-called central volume principle $M T T=\frac{C B V}{C B F}$. In perfusion imaging the CBF indicates the volume of blood that flows through a voxel per unit of time, the CBV how much blood is in that voxel, and the MTT the average time it takes the blood to flow through the voxel. In an ischemic stroke, the infarct core shows reduced CBF and CBV. In the infarct penumbra, the CBF is reduced, but the CBV is still normal or even slightly increased[19].

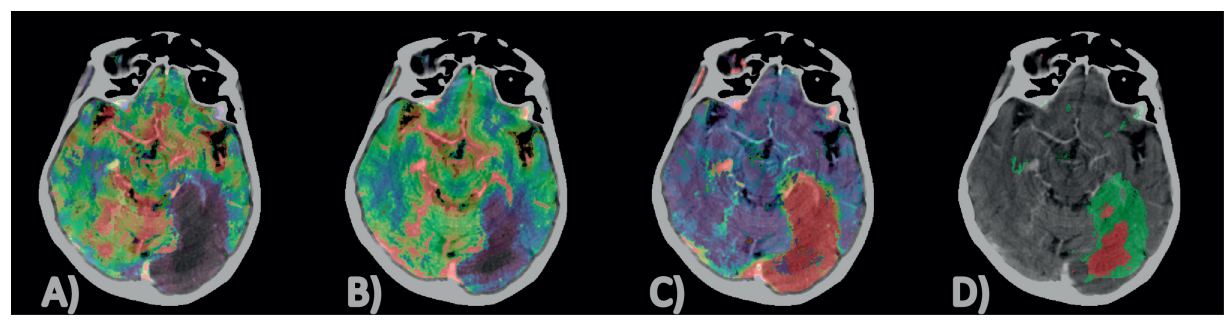

Figure 1.4 | CT perfusion maps. Parameter maps of a patient showing A) cerebral blood flow, B) cerebral blood volume, C) mean transit time and D) a so-called summary map, in which penumbra is highlighted in green and core in red. A clear ischemic area is observed in the left hemisphere (on the right in the image).

\section{CT angiography}

The third acquisition in the acute stroke protocol is CT angiography (CTA). This is also a contrast-enhanced CT scan but taken at a single time point (arterial phase) and with more dose, resulting in a higher signal-to-noise ratio and allowing assessment of the vasculature. The CTA is used to visualize occlusions of arteries, but it may also identify the cause of an occlusion e.g. cardiac thrombus, atherosclerosis or dissection[20]. 


\section{Dual-energy CT}

CT scanners employ X-ray tubes that emit a range or spectrum of photon energies typically between $50 \mathrm{keV}$ and $140 \mathrm{keV}$. Conventional CT imaging differentiates between different materials based on their $\mathrm{X}$-ray attenuation averaged for the photon energies and is usually shown in Hounsfield units (HU). Since the attenuation depends on both material density and material concentration, different tissue components can have similar HU, making differentiation between and classification of tissue types difficult[21].

Recent developments in CT technology offer the possibility to try and overcome these differentiation difficulties. Dual-energy CT (DECT) is a technique in which two independent attenuation measurements with different energy spectra are acquired[21], allowing the differentiation of materials that would otherwise have very similar HU such as iodine and calcium[22].

There are a number of technical approaches to acquire a dual-energy dataset, e.g. rapid $k V$ switching in which the $X$-ray tube switches between two energy $(k V)$ settings, dual sources in which two $X$-ray tubes are utilized that can operate at two different energy ( $\mathrm{kV}$ ) settings, or a dual-layer detector which separates the original X-ray spectrum in two spectra[21] (Figure 1.5). Conventionally a single-layer detector (SLCT) is employed that integrates all photon energies. All dual-energy systems enable adding additional features to conventional CT imaging, such as virtual monoenergetic images at discrete energy levels between 40 and 200 keV[23,24], virtual non-contrast CT[25,26], or material decomposition[27], thereby overcoming some of the differentiation and classifications difficulties.
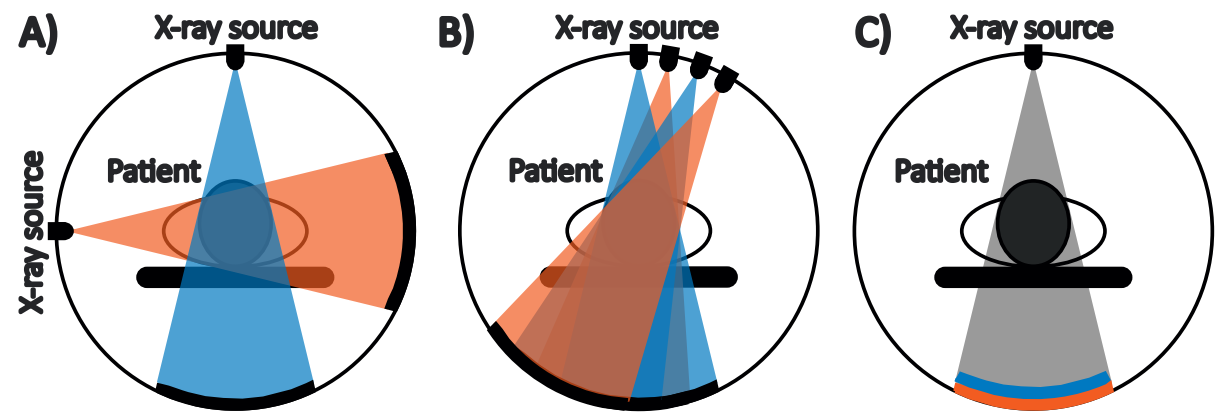

Figure 1.5 | Illustration of three dual-energy CT techniques. A) Dual-source DECT utilizes two $X$-ray tubes and two detectors to generate low- and high-energy spectra, B) rapid kV switching utilizes one X-ray tube and detector and switches rapidly between a low and high tube voltage and C) detector-based spectral CT utilizes one X-ray tube, and separates a single spectrum in a low- and high-energy dataset using a dual-layer detector. 
In stroke imaging, the increased differentiation between tissues by the use of monoenergetic images from dual-energy data[28] can be of interest in the detection of early ischemic changes since hypoattenuation due to edema could be better differentiated from normal brain tissue. In addition, for CTP, virtual monoenergetic images at low keV enhance iodine contrast and may improve CTP analysis. These applications could contribute to improved detection of ischemic stroke.

\section{Drawbacks of CT}

While CT enables anatomical and sometimes functional evaluation of imaged regions in the body, it does have a major drawback. CT imaging requires ionizing radiation, which can induce a minor increase in risk of acquiring cancer[29]. Accordingly, for the acute stroke protocol, we aim to keep X-ray dosage as low as possible. This however results in high amounts of noise in the $C T$ acquisitions. To reduce some of the noise in CTP imaging, the CTP scan is reconstructed to thick (5 to $10 \mathrm{~mm}$ ) slices. This however means that the sensitivity of CTP for small-volume infarcts is limited. To increase the sensitivity for small-volume infarcts, high-resolution thin-slice CTP is required. Because of the associated higher noise levels of thin-slice acquisitions, more robust analysis methods such as model based non-linear regression and advanced filter techniques[17] are needed. For NCCT a similar problem exists, since contrast differentiation between healthy and ischemic tissues is limited owing to the presence of noise and the low image resolution.

\section{ENCLOSE study}

In the context of the problems highlighted above, the "early detection of thromboembolic sources and small volume stroke with spectral CT" (ENCLOSE) study was initiated. ENCLOSE is a prospective multicenter cohort study that was funded by the Dutch Heart Foundation and Technology Foundation STW (project number: 14732), as part of their joint strategic research program: "Earlier recognition of cardiovascular diseases". The ENCLOSE's primary aims were to investigate the possibilities of DECT in 1) stroke imaging and 2) stroke etiology, 3) improve the detection of small-volume infarction with thin-slice CTP and 4) improve risk assessment for recurrent stroke in small-volume stroke and TIA patients. Aim 1 and aim 3 of ENCLOSE are addressed in this thesis. 


\section{Thesis outline}

This thesis will focus on the possibilities of high-resolution CT perfusion and DECT in CT stroke imaging. By aiming to improve the diagnostic performance for ischemia detection using DECT and improve CT perfusion analysis using DECT and high-resolution thinslice CT perfusion.

This thesis consists of three parts. Part I is concerned with comparing the technical aspects of DECT with conventional CT imaging. Chapter $\mathbf{2}$ determines the radiation dose associated with dual-layer detector CT (DLCT) and assesses how this compares with an otherwise technically equivalent single-layer CT (SLCT) over a broad range of clinical protocols. Chapter $\mathbf{3}$ investigates the effects of using DLCT on the image quality of conventional CT images in comparison with SLCT. In Chapter 4, non-contrast DECT virtual monochromatic images (VMI), reconstructed from a dual-source dual-energy CT (DSCT) scanner, are compared with conventional CT using image quality measures such as signal-to-noise ratio and contrast-to-noise ratio.

In part II, the added value of DECT compared with conventional CT in stroke imaging is evaluated using observers. In Chapter 5, an observer study evaluates non-contrast DECT VMI, scanned on a DSCT scanner, for the detection of cerebral ischemia in patients with concern for acute ischemic stroke. Chapter 6 investigates the use of virtual ischemia maps to increase the sensitivity for size and location of infarction on non-contrast DECT using DSCT. In Chapter 7, an observer study evaluates the quality of perfusion maps from VMI CTP using DLCT and is compared with the current clinical standard CTP.

Part III focusses on the technical aspects of CTP in CT stroke imaging. Chapter $\mathbf{8}$ investigates the effect of different acquisition intervals on quantitative perfusion maps and infarct volumes of three CTP analysis methods. In Chapter 9, high-resolution thinslice CT perfusion for detection of small-volume infarcts is evaluated.

Chapter 10 summarizes the presented research, and will provide a general discussion. This chapter will end with future perspectives on (DE)CT and stroke imaging. 
Introduction | Chapter 1 



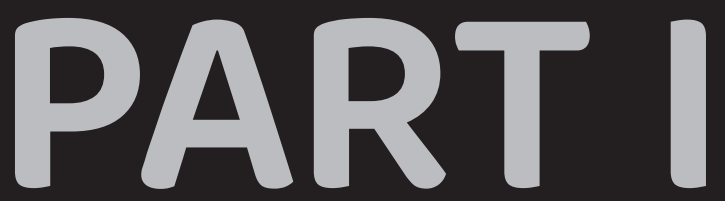

\section{TECHNICAL ASPECTS OF DUAL-ENERGY CT}





\title{
CHAPTER 2
}

\section{RADIATION DOSE OF A DUAL-LAYER CT SCANNER}

\author{
Based on
}

F van Ommen, HWAM de Jong, JW Dankbaar, E Bennink, T Leiner and AMR Schilham (2019). Dose of CT protocols acquired in clinical routine using a dual-layer detector

CT scanner: a preliminary report. European Journal of Radiology, 112:65-71.

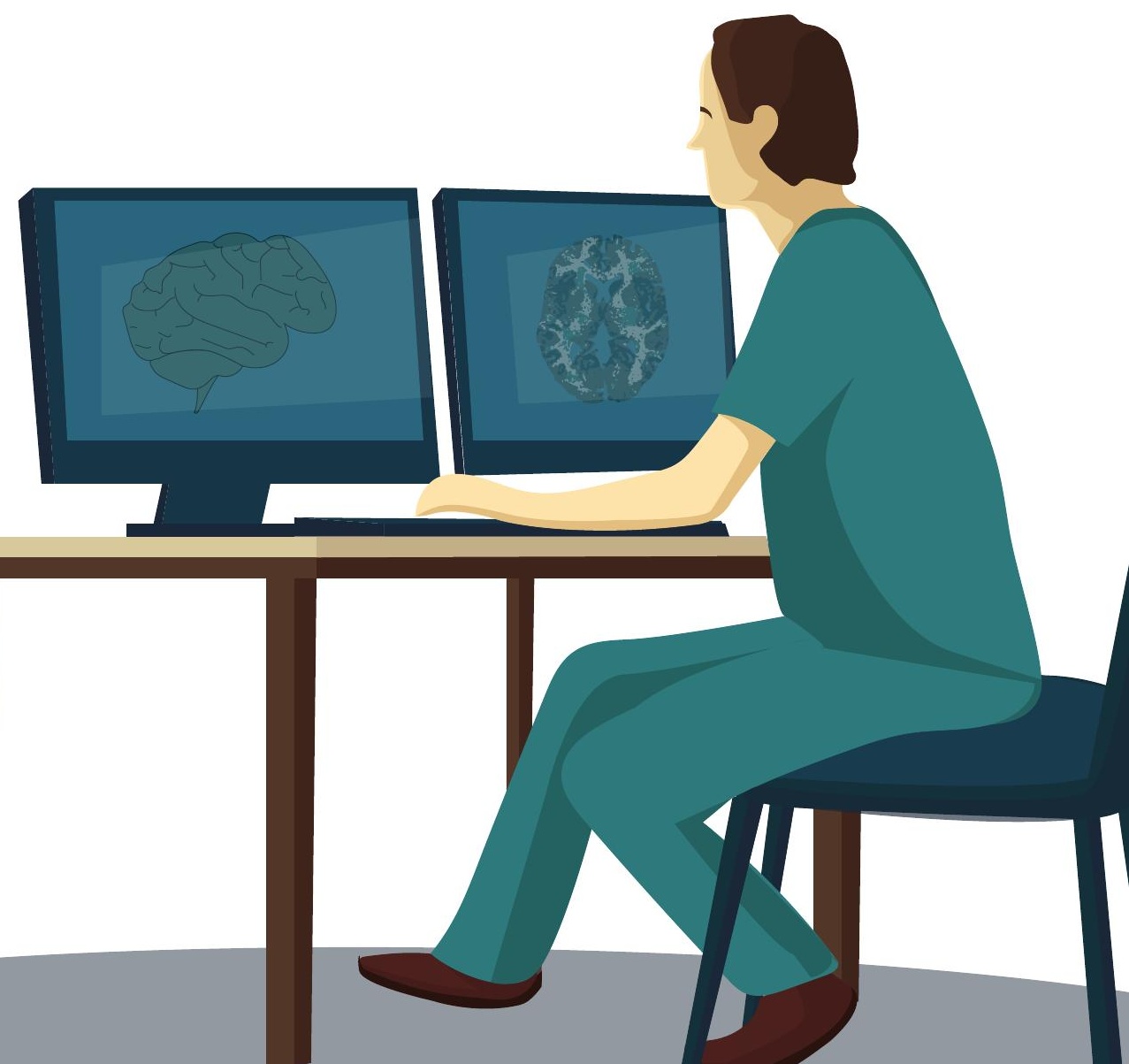


Part I | Technical aspects of dual-energy CT

\section{Abstract \\ Objectives}

To assess the radiation dose associated with always-on dual-energy acquisitions in clinical practice over a broad range of clinical protocols using dual-layer detector CT (DLCT; IQon Spectral CT, Philips Healthcare) as compared to an otherwise technically equivalent single-layer detector CT (SLCT; Brilliance iCT, Philips Healthcare).

\section{Methods}

Dose-length-product data for consecutive examinations over a six-month period acquired with DLCT were retrospectively collected and compared to consecutive examinations from an SLCT. Imaging protocols were optimized for diagnostic image quality for each system prior to data collection. Dose reports of CT protocols that were used at least 50 times on both systems were collected. After exclusion of statistical outliers, protocols were evaluated with regard to reported dose levels.

\section{Results}

In total, 4536 dose reports for DLCT and 5783 reports for SLCT were collected. All DLCT examinations were acquired at $120 \mathrm{kVp}$, enabling dual-energy analysis. With SLCT, $79 \%$ of examinations were acquired at $120 \mathrm{kVp}$, and 21\% at 100/80 kVp. Protocols for 15 indications were used more than 50 times on both scanners. For seven protocols there was no significant difference between the two scanners ( $p>0.07)$, whereas seven protocols were acquired with higher dose levels on SLCT compared to the DLCT $(p<0.001)$. For one protocol, the DLCT dose was significantly higher $(p=0.003)$ compared to the SLCT.

\section{Conclusions}

Dual-layer detector CT enables acquisition of dual-energy information over a broad range of clinical indications without increasing radiation dose when compared to a conventional single-layer detector CT. 


\section{Introduction}

Dual-energy CT (DECT) is a technique in which two attenuation measurements are obtained at two different energies. DECT provides additional information, and allows for the differentiation and quantification of material composition[21] and can be used to improve image quality[30-34]. A number of dedicated DECT techniques have been commercially introduced over the past few years, including rapid $\mathrm{kV}$ switching and dual X-ray sources. These approaches acquire two attenuation datasets by separating energies at the tube level. For most patient exams, however, these dual-energy systems are operated as conventional scanners, because dual-energy scanning has an impact on scanner performance[21,35] or increases radiation dose[36,37].

Recently, a dual-layer detector CT (DLCT) system (IQon Spectral CT, Philips Healthcare, Best, the Netherlands) has become clinically available. The DLCT detector allows differentiation of the two datasets at the detector level. With a DLCT the choice for either dual-energy or conventional acquisitions is no longer necessary, since every acquisition is a dual-energy acquisition from which both conventional and dual-energy CT images can be reconstructed. To acquire the two datasets, however, the acquisition needs to be acquired at high tube voltages (i.e. 120 or $140 \mathrm{kVp}$ ). In addition, since the separation of the energy spectrum occurs at the detector level, an increase in lowenergy photon flux is needed to improve spectral separation, which is achieved by reducing the titanium filtering on the DLCT compared to a single-layer detector CT (SLCT; Brilliance iCT, Philips Healthcare, Best, the Netherlands). These adaptations both entail changes in the energy spectrum of the DLCT compared to the SLCT and may have implications for the radiation dose patients receive. These changes in the energy spectrum raise the question; does the additional information from the DLCT come at the cost of additional dose to the patient compared to a conventional CT acquisition conducted on a SLCT?

The objective of this study was to assess the radiation dose associated with always-on dual-energy acquisitions in daily clinical practice over a broad range of clinical protocols using dual-layer spectral CT (IQon Spectral CT) as compared to an otherwise technically equivalent single-layer detector CT scanner (Brilliance iCT).

\section{Materials and Methods}

In this retrospective study, dose-length-product (DLP) data for consecutive examinations over a six-month period acquired with DLCT were collected and compared to consecutive examinations performed on a SLCT. Our retrospective study was approved by the local ethics committee and waived the need for informed consent (ethical number: 18-162/C). To study dose levels of the DLCT in clinical practice, a comparison was made by comparing dose levels of clinically scanned CT protocols on both scanners in retrospect. 
The SLCT was selected, because the DLCT and SLCT are technically equivalent scanners, with exception of the detector and the difference in X-ray beam filtration.

\section{Clinical data selection}

CT protocols on the DLCT scanner were made by adapting CT protocols of the SLCT. The conversion of clinical protocols from 80 or $100 \mathrm{kVp}$ acquisitions to $120 \mathrm{kVp}$ was based on CTDI matching. Additional modifications to protocol parameters were made to achieve diagnostic image quality, and similar noise levels.

At our institute a dose report of every patient exam is stored in an OpenREM database[38]. In this database patient age, sex, CT protocol description, number of acquired scans and total DLP of the CT exam are stored. Data collected from this database served as input for the present study. For both CT systems, all dose reports for examinations over a continuous six-month period were collected. CT protocols eligible for inclusion in this study were protocols acquired at least 50 times in the selected time period on both CT scanners. For this comparison examinations acquired from 01-08-2017 until 31-01-2018 were included. With the introduction of the DLCT system, a shift of performing certain examinations from the SLCT to the DLCT was observed. To compensate for this shift, the time period for the SLCT system was set to 01-01-2017 to 30-06-2017.

In clinical practice, a number of examinations were terminated before all acquisitions of the protocol were completed. Furthermore, some examinations required additional acquisitions due to e.g. artifacts or additional request from the treating physician. To exclude these non-standard examinations from the analysis, the interquartile range (IQR) rule was applied to the data[39]; values above $Q 3+1.5 * I Q R$ and values below $Q 1-1.5 * I Q R$ were considered outliers and removed from the analysis. $Q 1$ and $Q 3$ are the first and third radiation dose quartile $(Q)$ and $I Q R=Q 3-Q 1$.

\section{Phantom experiments}

To verify doses reported by the CT scanners dose measurements in phantoms were conducted. The DLP was measured with a 10 centimeter pencil ionization chamber (Raysafe, Billdal, Sweden) for body and head scans using a body and head phantom (IBA dosimetry, Bartlett, TN, USA). The measured DLP was subsequently compared to the reported DLP on the scanner. Dose was compared at 80, 100, 120 and $140 \mathrm{kVp}$ with an exposure of 200 mAs. All other acquisition parameters (e.g. collimation, rotation time, scan arc etc.) were kept the same on both systems.

\section{Statistical analysis}

Radiation dose of the CT protocols obtained with both systems were visually compared using boxplots. Statistical analysis was performed by two readers (FvO and AS). For statistical analysis of the data MATLAB (MathWorks, Natick, MA, USA) was used. 
Differences in dose between the two different CT systems were assessed using an unpaired sample t-test. The $p$-value was set at 0.0033 to account for multiple sampling (Bonferroni correction).

\section{Results}

\section{Clinical data selection}

In the selected time period a total of 4536 dose reports were collected from the DLCT. A total of 16 protocols were used more than 50 times (range: 52 - 861). On the SLCT a total of 5783 dose reports were collected, and 24 protocols were scanned more than 50 (range: 55 - 1340) times. After exclusion of outliers, 15 protocols were scanned more than 50 times (range: 53 - 1099) on both systems. In Table 2.1 these protocols are broken down into five anatomical categories.

Table 2.1 | CT protocols scanned at least 50 times on both scanners.

\begin{tabular}{lllll}
\hline Head and Brain & Neck & Cardiac & Chest & Abdomen \\
\hline CT Brain & CT Neck & CT Heart & CT Chest & CT Abdomen \\
CT Brain & CT Cervical spine & CTA Pulmonary & CT non-contrast & CT Intravenous \\
perfusion & & arteries & chest & pyelography \\
CTA Circle Willis & & $\begin{array}{l}\text { CT High-resolution } \\
\text { chest }\end{array}$ & CT Kidney stones \\
CTA Carotids & & & CTA Abdomen \\
\hline
\end{tabular}

All DLCT examinations were acquired at $120 \mathrm{kVp}$. For the SLCT 79\% of examinations were acquired at $120 \mathrm{kVp}$ and $21 \%$ at 100 or $80 \mathrm{kVp}$. Six protocols on the DLCT included acquisitions in which the tube voltage was increased to be able to acquire dual-energy datasets (i.e. CT brain perfusion, CTA of the carotid and cerebral vasculature, CTA of pulmonary arteries, high-resolution CT of the chest, CT intravenous pyelography and $\mathrm{CT}$ of kidney stones). In the remaining 9 protocols, the tube voltages were already at $120 \mathrm{kVp}$ on the SLCT system.

\section{Phantom experiments}

The difference between the measured and reported DLP on the DLCT was within maximally $4.0 \%$ for both body and head scans (Table 2.2). The reported DLP was underestimated at all kVp, except for the $140 \mathrm{kVp}$ body scans. Generally, the difference on the SLCT was slightly higher (Table 2.2) compared to the DLCT. In all cases, however, the measured DLP was still within 7.5\% of the reported DLP. The SLCT underestimated the reported dose compared to the measured dose. 
Part I | Technical aspects of dual-energy CT

Table 2.2 | Reported and measured DLP (mGy/cm) at different tube voltages for body and head scans for the DLCT and SLCT. The difference is given in \%. Dose was measured at $200 \mathrm{mAs}$, with $64 \times 0.625 \mathrm{~mm}$ collimation.

\begin{tabular}{|c|c|c|c|c|c|c|c|c|c|c|c|c|}
\hline & \multicolumn{6}{|c|}{ DLCT } & \multicolumn{6}{|c|}{ SLCT } \\
\hline & \multicolumn{3}{|c|}{ Body } & \multicolumn{3}{|c|}{ Head } & \multicolumn{3}{|c|}{ Body } & \multicolumn{3}{|c|}{ Head } \\
\hline & Meas. & Rep. & Diff. & Meas. & Rep. & Diff. & Meas. & Rep. & Diff. & Meas. & Rep. & Diff. \\
\hline $80 \mathrm{kVp}$ & 24.52 & 24.0 & 2.12 & 47.4 & 47.6 & -0.42 & 18.16 & 16.8 & 7.49 & 36.00 & 34.8 & 3.33 \\
\hline $100 \mathrm{kVp}$ & 47.48 & 45.6 & 3.96 & 89.24 & 88.8 & 0.54 & 37.80 & 35.2 & 6.88 & 70.72 & 70.0 & 1.02 \\
\hline 120 kVp & 75.28 & 72.4 & 3.77 & 136.64 & 137.2 & -0.41 & 60.72 & 58.8 & 3.16 & 116.72 & 114.8 & 1.64 \\
\hline $140 \mathrm{kVp}$ & 103.64 & 104.0 & -0.35 & 193.40 & 194.4 & -0.52 & 88.00 & 86.4 & 1.82 & 172.60 & 167.6 & 2.90 \\
\hline
\end{tabular}

Abbreviations: Meas.; measured DLP, Rep.; reported DLP and Diff.; difference between the measured and reported DLP.

\section{Radiation dose per anatomical region}

Head and Brain

In Figure 2.1, DLP of the head and brain protocols are shown, corrected for outliers. For the CT brain perfusion and CTA of the carotid and cerebral vasculature acquisition in the CT perfusion and CTA Circle Willis protocol the peak kilovoltage was changed from 80 or $100 \mathrm{kVp}$ to $120 \mathrm{kVp}$ on the DLCT to facilitate acquisition of dual-energy datasets. For both these protocols the radiation dose on the SLCT was slightly higher compared to the DLCT $(p<0.001$, Table 2.3). The brain CT and CTA of the carotids protocols showed near identical dose levels for both scanners. The difference between the two was not significant $(p>0.27$, Table 2.3). For CTA of the carotid and cerebral arteries a large spread in observed dose levels was found. To illustrate the CT images typically observed with a brain CT scan, we have provided a CT brain scan from the DLCT and the SLCT in Figure 2.2.

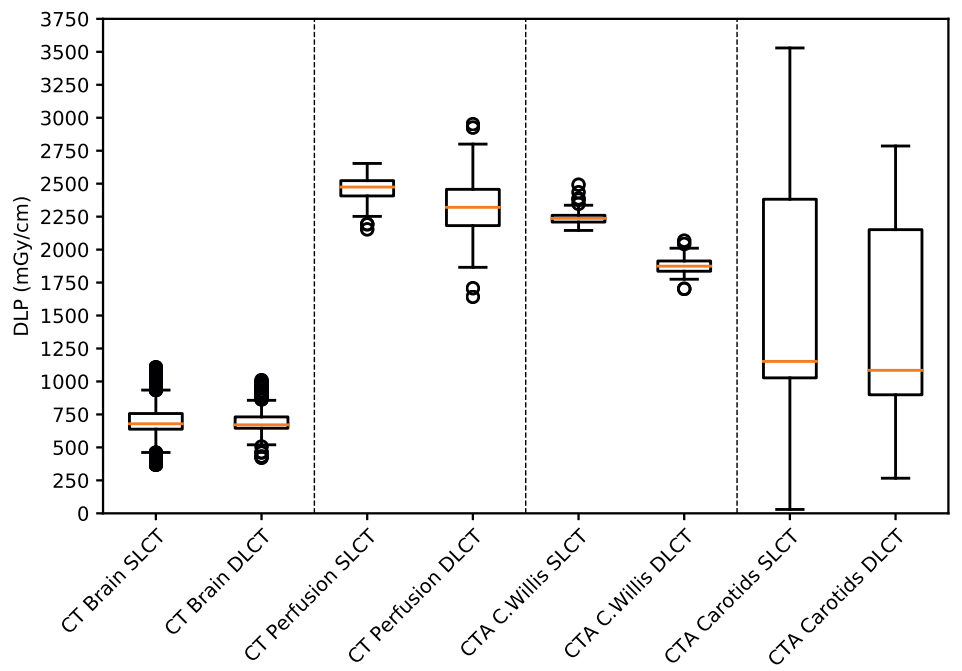

Figure 2.1 | Dose levels (DLP) of head and brain CT protocols on the SLCT and DLCT. CT Brain perfusion is abbreviated with CT perfusion, CTA Circle Willis with CTA C.Willis and CTA of the carotids and cerebral arteries with CTA carotids. Outliers are marked by circles. 
Table 2.3 Mean dose levels (DLP) of indications scanned more than 50 times, with the mean dose difference (\%) and the $p$-value. The $p$-value is marked with an asterisk when a significant difference was found. The highest mean dose was highlighted bold.

\begin{tabular}{|c|c|c|c|c|c|c|}
\hline \multirow[b]{2}{*}{ CT protocol } & \multicolumn{2}{|c|}{ DLCT (IQon) } & \multicolumn{2}{|c|}{ SLCT (iCT) } & \multirow[b]{2}{*}{ Diff. (\%) } & \multirow[b]{2}{*}{ P-value } \\
\hline & Total & Dose (DLP) & Total & Dose (DLP) & & \\
\hline CT Brain & 715 & 696.58 & 1099 & 702.12 & 0.79 & 0.279 \\
\hline СТ Brain perfusion & 168 & 2317.35 & 168 & 2463.59 & 5.94 & $<0.001 *$ \\
\hline CTA Circle Willis & 100 & 1878.66 & 91 & 2241.68 & 16.19 & $<0.001^{*}$ \\
\hline CTA Carotid and cerebral arteries & 66 & 1376.67 & 88 & 1480.10 & 6.99 & 0.418 \\
\hline CT Neck & 92 & 641.30 & 82 & 573.56 & -11.81 & 0.121 \\
\hline CT Cervical spine & 147 & 365.47 & 135 & 292.84 & -24.80 & 0.072 \\
\hline CT Heart & 110 & 226.72 & 53 & 204.78 & -10.71 & $0.003 *$ \\
\hline CTA Pulmonary arteries & 262 & 167.46 & 219 & 226.35 & 26.01 & $<0.001 *$ \\
\hline CT Chest & 702 & 563.03 & 496 & 571.65 & 1.51 & 0.612 \\
\hline CT non-contrast Chest & 184 & 81.06 & 123 & 135.07 & 39.98 & $<0.001^{*}$ \\
\hline CT High-resolution Chest & 147 & 97.11 & 92 & 163.16 & 40.48 & $<0.001 *$ \\
\hline CT Abdomen & 341 & 575.97 & 607 & 641.34 & 10.19 & $<0.001 *$ \\
\hline CT Intravenous pyelography & 75 & 520.73 & 106 & 668.55 & 22.11 & $<0.001^{*}$ \\
\hline CT Kidney stones & 80 & 280.83 & 56 & 301.00 & 6.70 & 0.330 \\
\hline CTA Abdomen & 72 & 1234.22 & 77 & 1387.01 & 11.02 & 0.141 \\
\hline
\end{tabular}

Abbreviations: Diff.; mean dose difference.

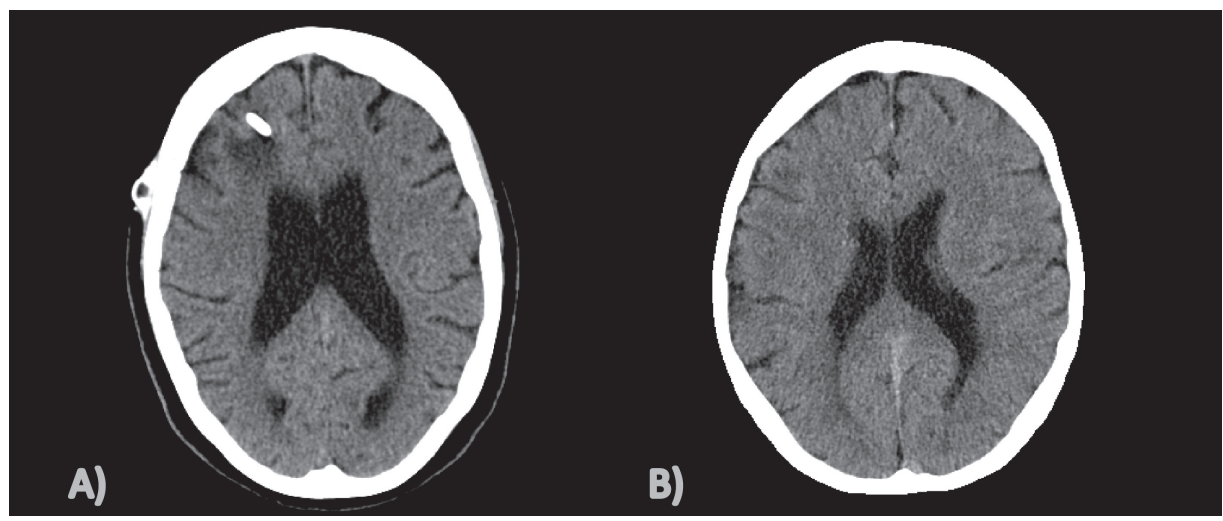

Figure 2.2 $\mid$ Characteristic images of the CT brain protocol from A) the SLCT and B) DLCT. The contrast between the gray and white matter is clear in both images. In the SLCT image, a drain is visible. The window level and width for both images is $40 / 80 \mathrm{HU}$.

Neck

For neck and cervical spine examination the DLP was slightly higher on the DLCT compared to the SLCT (Figure 2.3). The difference, however, was not significant ( $p>0.07$, Table 2.3). For both scanners a large spread in dose levels was observed. 


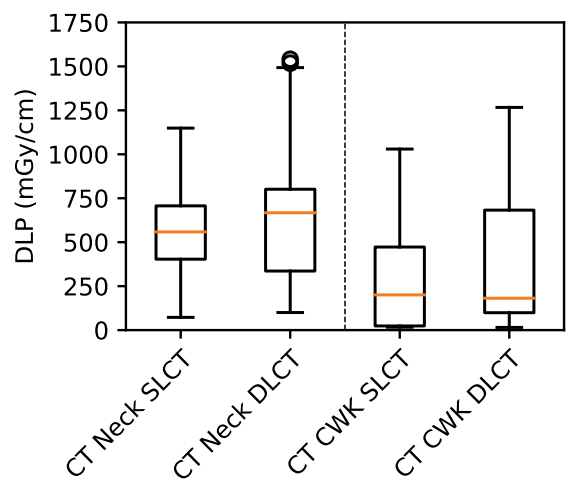

Figure 2.3 | Dose levels (DLP) of neck CT protocols on the SLCT and DLCT. CWK stands for cervical spine CT protocols. Outliers are marked by circles.

\section{Cardiac}

Mean radiation dose levels in the cardiac examinations are shown in Figure 2.4. The pulmonary artery CTA protocol was changed from $80 \mathrm{kVp}$ to $120 \mathrm{kVp}$ to enable routine acquisition of dual-energy datasets. Mean dose levels on the SLCT were slightly lower compared to the DLCT for the Heart CT protocol. This difference was significant $(p<0.005$, Table 2.3). The CTA Pulmonary exams illustrated the opposite of this, in which the DLCT was slightly lower compared to the SLCT ( $p<0.001$, Table 2.3).

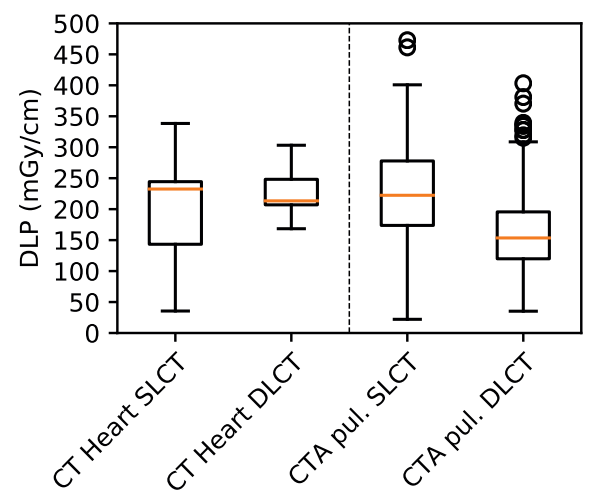

Figure 2.4 | Dose levels (DLP) of neck CT protocols on the SLCT and DLCT. CWK stands for cervical spine CT protocols. Outliers are marked by circles.

\section{Chest}

In Figure 2.5, radiation dose levels of the chest CT protocols are shown. The highresolution (HR) chest CT protocol was changed from $100 \mathrm{kVp}$ to $120 \mathrm{kVp}$ to be able to acquire the dual-energy datasets. 
For both the non-contrast chest CT and the HR chest CT a small, but significant difference was observed between the SLCT and DLCT $(p<0.001$, Table 2.3), with slightly lower mean radiation dose levels observed on DLCT versus SLCT. The contrast enhanced chest CT protocol displayed comparable dose levels, which did not show a significant difference ( $p>0.61$, Table 2.3 ). The protocol varied widely with regard to dose levels.

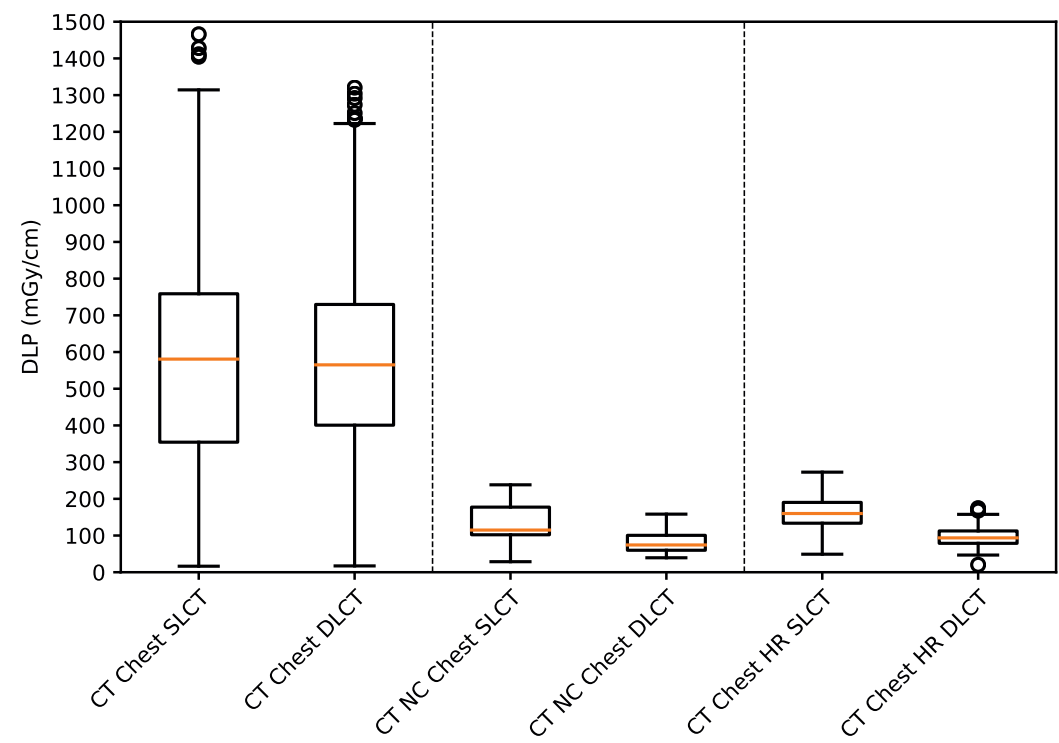

Figure 2.5 Dose levels (DLP) of chest CT protocols on the SLCT and DLCT. CT NC chest represents the non-contrast chest CT protocol. Outliers are marked by circles.

\section{Abdomen}

Radiation dose levels of abdominal CT protocols after outlier correction are shown in Figure 2.6. For both the CT intravenous pyelography and kidney stones protocol the peak kilovoltage of the non-contrast CT was changed from $100 \mathrm{kVp}$ to $120 \mathrm{kVp}$. In general, the radiation dose of the SLCT protocols was slightly higher compared to the DLCT. For the intravenous pyelogram this difference was significant $(p<0.001$, Table 2.3), whereas for the kidney stones protocol the difference was not significant ( $p>0.33$, Table 2.3). Mean dose levels of the abdomen CT protocol were significantly higher on the SLCT compared to the DLCT ( $p<0.001$, Table 2.3). Lastly, mean radiation dose levels for CTA of the abdomen exams did not show significant differences between both systems ( $p>0.14$, Table 2.3). However, a large variation in dose levels was observed. Similarly to the brain scan, in Figure 2.7, an example of a typical CT scan of the abdomen is shown. 


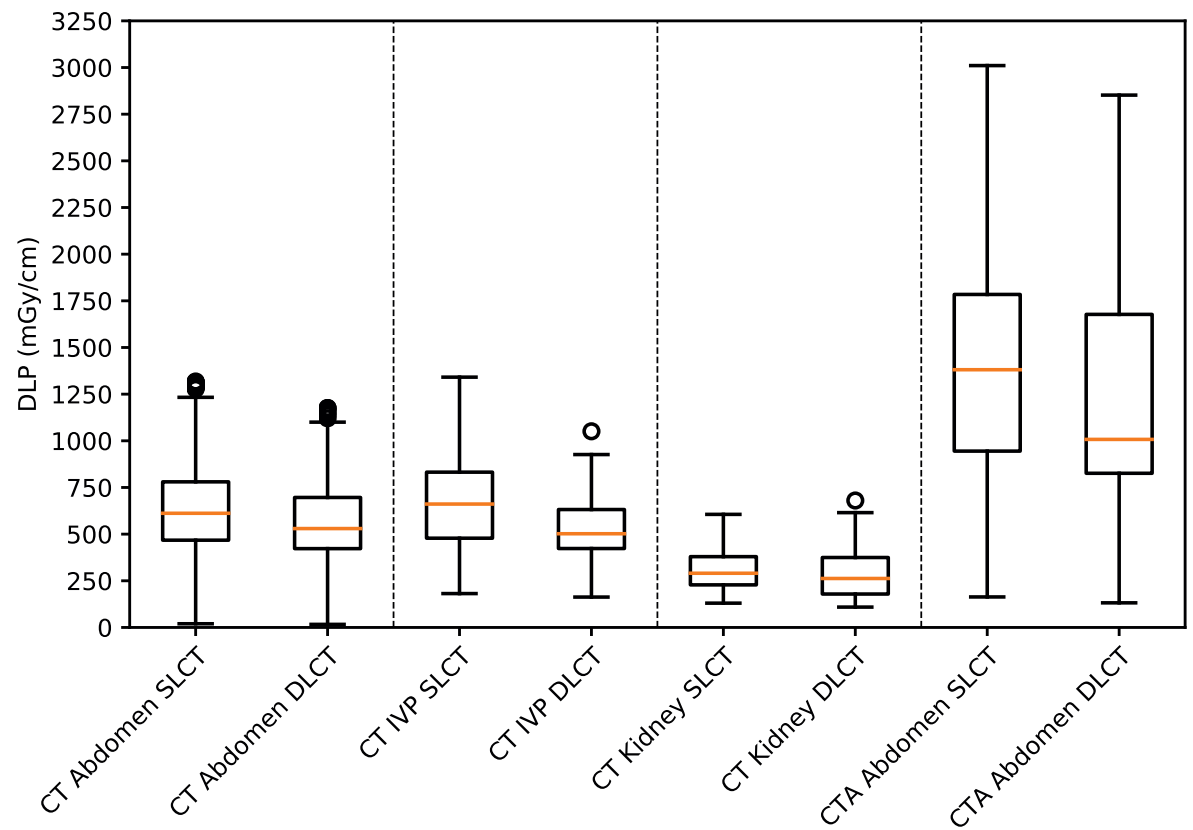

Figure 2.6 | Dose levels (DLP) of abdomen CT protocols on the SLCT and DLCT. In which IVP stands for intravenous pyelography and Kidney for kidney stones CT protocols. Outliers are marked by circles.

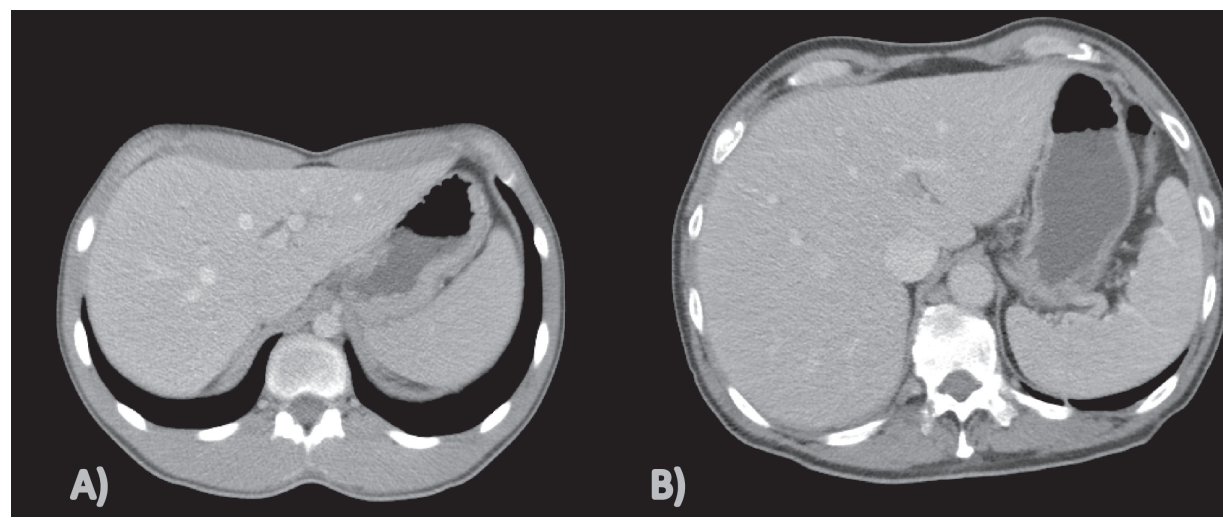

Figure 2.7 | Characteristic images of the CT abdomen protocol from A) the SLCT and B) DLCT. In both images the liver, intestines, spleen and ribs are clearly visible. The window level and width for the images is $25 / 400 \mathrm{HU}$. 


\section{Discussion}

In this retrospective study, we assessed the radiation dose of always-on DLCT dualenergy acquisitions in daily clinical practice over a broad range of clinical indications by comparing dose levels with a technically equivalent SLCT system. In general, dose levels of CT protocols scanned on the DLCT were equivalent to or lower than the dose levels on the SLCT.

Our results extend the current literature on dose levels when using a DLCT system. A number of studies have been published on applications of DLCT dual-energy analysis[30,40-47]. These studies, however, disregard dose levels or omitted a comparison to conventional SLCT system. In only two instances the dose levels of chest[40] and abdominal[41] CTs were mentioned, in which the dose levels were found to be equivalent or significantly lower compared to the SLCT. To establish the radiation dose associated with use of $120 \mathrm{kVp}$ DLCT in clinical routine a direct comparison of dose levels was merited.

The differences between measured and reported dose on the DLCT and SLCT were 7.5\% at most. The difference was slightly higher on the SLCT compared to the DLCT. These differences, however, are not substantial. Therefore, we used the reported DLPS without correction in our analysis.

For a number of CT protocols (i.e. CTA carotid and cerebral arteries, CT neck, CT cervical spine, CT chest and CTA Abdomen), we observed a large variation in dose levels. There are two reasons for this variation. First, we compared CT protocols based on protocol name, and not on clinical question. We have a number of standard protocols, and depending on the clinical question such a protocol is extended or scans are simply omitted to save dose, which results in a large variation in observed dose levels. We consider this a limitation of this study; however, we do believe that this comparison gives a reliable insight in dose levels in a clinical setting of the DLCT, since a protocol was included when it was completed at least 50 times and statistical outliers were removed before the analysis. Secondly, the use of automatic exposure control (AEC) also results in a larger IQR. Philips Healthcare utilizes a reference-image based AEC, in which a topogram is used to assess the patient's attenuation to set the tube current[48]. AEC aims to reduce radiation exposure to the patient, due to large inter-patient variability; large differences are observed in tube current settings between patients resulting in substantial dose differences. 
Seven out of 15 protocols we compared did not show any significant differences. For seven protocols, radiation dose on the SLCT was significantly higher compared to 120 kVp DLCT. The difference for the CT brain perfusion and the CTA of the circle of Willis protocol can be attributed to the fact that on the SLCT the CT perfusion acquisition consists of 30 images, whereas on the DLCT only 22 images. This exam required a technique called Jog Mode to extend the spatial coverage from 40 to $80 \mathrm{~mm}$ to match the spatial coverage of the SLCT, subsequently increasing the temporal sampling interval from two to 3.4 seconds per image. For the non-contrast chest CT and CT Abdomen, a small, but significant difference was found. This difference is most likely due to the use of AEC in the acquisition. Since, these protocols were already imaged at hightube voltage on the SLCT. In the pulmonary artery CTA, the HR chest CT and the CT Intravenous pyelography protocol a significant difference in favor of the DLCT was observed. These protocols were for exams in which some of the acquisitions were changed from a low-tube voltage to a high-tube voltage, which demonstrates that dose does not necessarily increases with the acquisition of high kVp dual-energy CT datasets instead of low kVp conventional CT. The dose on the DLCT was significantly higher for the CT heart protocol compared to the SLCT. This small difference can be attributed to a slightly higher exposure in the locator and tracker for the CTA acquisition for the DLCT protocol compared to the SLCT.

This study has another limitation. We did not objectively measure the image quality of the acquired CT images. However, prior studies found that conventional CT images from the DLCT and SLCT are of comparable image quality[41,49,50]. In addition, these scans were used clinically; we therefore concluded that the acquired images are of sufficient image quality for routine diagnostic evaluation. For further research, an extensive evaluation of image quality in the routine clinical images should be considered. Lastly, we did not perform patient based radiation monitoring, but rather used the dose report of the CT scanner itself, which we consider another limitation of our study.

In conclusion, DLCT, using a $120 \mathrm{kVp}$ tube potential, can be routinely used in daily clinical practice to provide additional information without increasing radiation dose when compared to conventional SLCT. 
Radiation dose of a dual-layer CT | Chapter 2 



\title{
CHAPTER 3
}

\section{IMAGE QUALITY OF CONVENTIONAL CT IMAGES ON A DUAL-LAYER CT SCANNER}

\author{
Based on
}

F van Ommen, E Bennink, A Vlassenbroek, JW Dankbaar, AMR Schilham, MA Viergever and HWAM de Jong (2018). Image quality of conventional images of dual-layer

SPECTRAL CT: A phantom study. Medical Physics, 45:3031-3042.

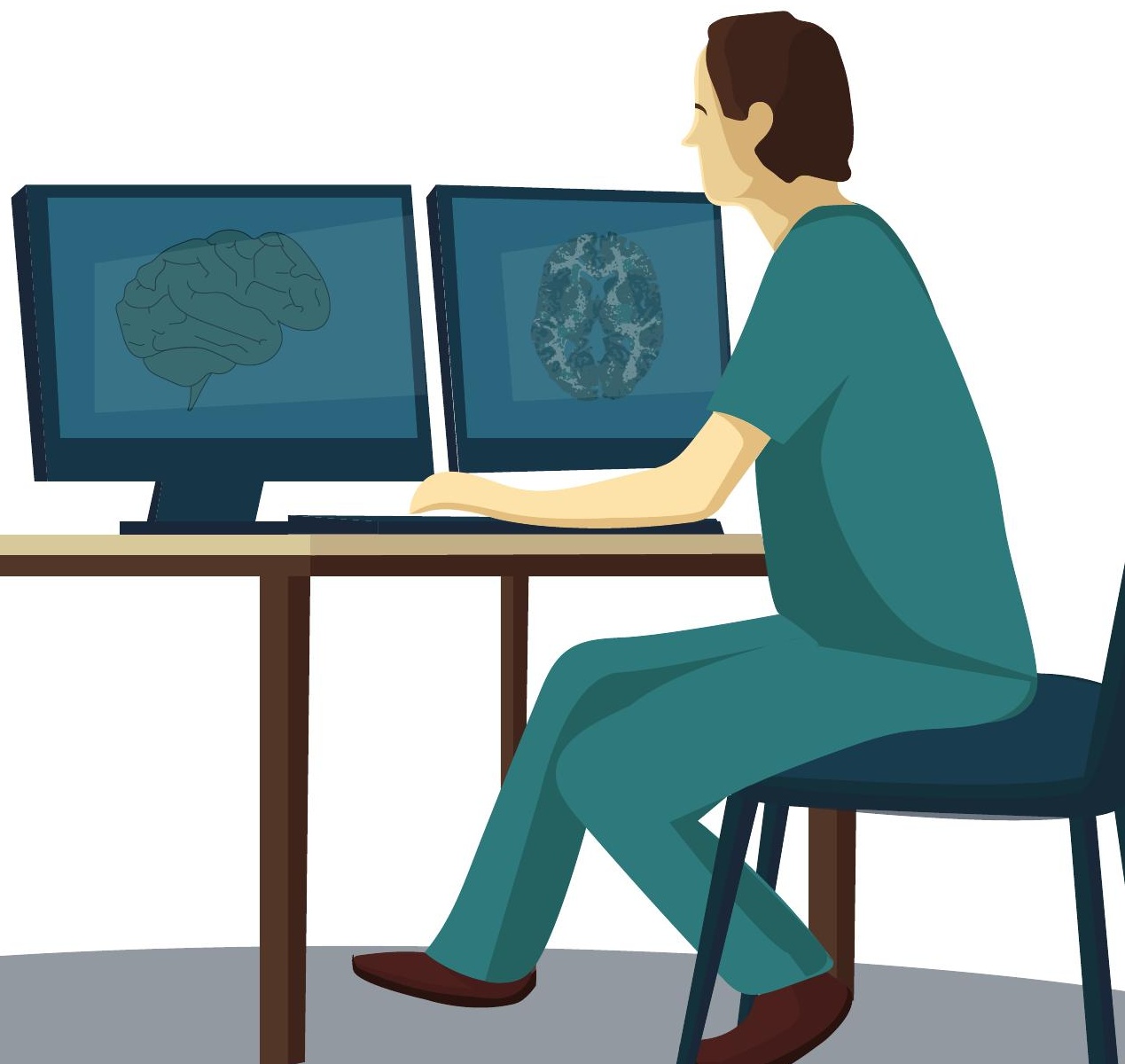


Part I | Technical aspects of dual-energy CT

\section{Abstract \\ Objectives}

Spectral CT using a dual-layer detector offers the possibility of retrospectively introducing spectral information to conventional CT images. In theory, the dual-layer technology should not come with a dose or image quality penalty for conventional images. In this study, we evaluate the influence of a dual-layer detector (DLCT; IQon Spectral CT, Philips Healthcare) on the image quality of conventional CT images, by comparing these images with those of a conventional but otherwise technically comparable single-layer CT scanner (SLCT; Brilliance iCT, Philips Healthcare), by means of phantom experiments.

\section{Methods}

For both CT scanners conventional CT images were acquired using four adult scanning protocols: body helical, body axial, head helical and head axial. A CATPHAN 600 phantom was scanned to conduct an assessment of image quality metrics at equivalent (CTDI) dose levels. Noise was characterized by means of noise power spectra (NPS) and standard deviation (SD) of a uniform region, and spatial resolution was evaluated with modulation transfer functions (MTF) of a tungsten wire. In addition, contrast-tonoise ratio (CNR), image uniformity, CT number linearity, slice thickness, slice spacing, and spatial linearity were measured and evaluated. Additional measurements of CNR, resolution and noise were performed in two larger phantoms.

\section{Results}

The resolution levels at 50\%, 10\% and 5\% MTF of the SLCT and DLCT showed small but significant differences up to $0.25 \mathrm{lp} / \mathrm{cm}$ for body scans, and up to $0.2 \mathrm{lp} / \mathrm{cm}$ for head scans in favor of the DLCT. The SLCT and DLCT showed perfect CT linearity for body scans, but for head scans both scanners showed an underestimation of the CT numbers of materials with a high opacity. Slice thickness was slightly overestimated for both scanners. Slice spacing was comparable and reconstructed correctly. In addition, spatial linearity was excellent for both scanners, with a maximum error of $0.11 \mathrm{~mm}$. CNR was higher on the DLCT compared to the SLCT for both normal and larger phantoms with differences up to 0.51. Spatial resolution did not change with phantom size, but noise levels increased significantly. For head scans, DLCT had a noise level that was significantly lower than the SLCT; on the other hand DLCT showed noise levels significantly higher than the SLCT for body scans. Still, these differences were well within the specified range of performance of SLCT scanners.

\section{Conclusions}

At equivalent dose levels, this study showed similar quality of conventional images acquired on SLCT and DLCT for medium-sized phantoms and slightly degraded image quality for (very) large phantoms at lower tube voltages on the DLCT. Accordingly, it may be concluded that the introduction of a dual-layer detector neither compromises image quality of conventional images nor increases radiation dose for normal-sized patients, and slightly degrades dose efficiency for large patients at $120 \mathrm{kVp}$ and lower tube voltages. 


\section{Introduction}

Dual-energy CT (DECT) has been an active research area since first investigated by Alvarez and Macovski in 1976[51,52]. However, owing to a number of technical difficulties DECT was not clinically applicable until recent advances in CT technology, and has since led to the introduction of several DECT systems. In DECT imaging two datasets are acquired at different energies. In this way DECT utilizes the property that materials have different attenuation coefficients at different energies, which allows for the differentiation of materials according to their attenuation characteristics[21].

A number of dedicated dual-energy techniques have been commercially introduced over the past few years. These include scanners with tube potential (kV) switching, dual X-ray sources, split X-ray beam, or a dual-layer detector. The first three types only allow for prospective dual-energy DECT, in which the choice of acquisition, i.e. conventional imaging or dual-energy imaging, has to be made in advance. The fourth type has a dedicated dual-layer detector which allows retrospective DECT imaging. In tube potential switching scanners, such as the Discovery CT750 (GE Healthcare, Chicago, IL, USA), and dual X-ray source scanners, such as the Somatom Force (Siemens Healthineers, Erlangen, Germany), a specific DECT protocol has to be selected before the start of the acquisitions. This on the one hand provides a low- and high-energy dataset, but on the other hand has its limitations with respect to conventional imaging. The main limitation for $\mathrm{kV}$ switching is that both energy scans are limited to the same filtration, and therefore have a relatively high overlap of the energy spectra, and for dual $X$-ray sources the main limitations are a reduced field-of-view and cross scattering[21,35]. These limitations may be acceptable, because the benefits of DECT imaging may outweigh the limitations in certain diagnostic examinations.

Dual-layer detector CT (DLCT) scanners, such as the IQon Spectral CT (Philips Healthcare, Best, the Netherlands), always acquire two datasets, independent of protocol[53]. In the DLCT, a single $X$-ray source is used to expose a detector, consisting of two scintillator layers. The top layer is more sensitive to the low-energy photons, whereas the bottom detector layer absorbs more of the high-energy photons. This essentially separates a single X-ray beam into two components[35]. The use of a dual-layer detector enables retrospective dual-energy analysis on every recorded dataset acquired at high tube voltage (e.g. 120 and 140 kVp). However, since in current clinical practice the dual-layer spectral CT scanner is mainly used for routine non-spectral imaging, these images should not come with a dose or image quality penalty compared to conventional CT images. The dual-layer technology allows the creation of conventional images, by virtue of the spatial and temporal alignment of the datasets from the two detector layers. 
This alignment allows adding up the sinograms from both layers for reconstruction of a conventional CT image, so that the detector essentially functions as a single-layer detector. In theory, this means that the data collected from the two layers is equivalent to the data collected with a single-layer detector, and thus hardly introduces any penalty regarding image quality. However, there are a number of substantial differences between the two detectors, which make this assumption not straightforward. An important factor is that the dual-layer detector has two electronic channels per detector element. Each read-out contributes electronical noise, resulting in increased higher electronical noise per detector element than in a single-layer detector with only one read-out. The electronic channels on the DLCT, however, have an improved low-dose performance compared with the older electronic channels, which reduces noise slightly per channel as compared with the singlelayer detector. In addition, the dual-layer detector has a higher X-ray stopping power, and a higher light output of the scintillators than the single-layer detector. Furthermore, the DLCT has a tungsten backbone. The backbone, does not only provide stability, but also reduces cross-talk. Lastly, the dual-layer detector has a slightly lower geometrical efficiency than a single-layer detector due to side read out. These differences all can influence image quality and dose efficiency, and raise the question to whether or not the dual-layer detector would perform similarly to a single-layer detector. The purpose of this study is to investigate the assumption that the acquisition on a dual-layer detector CT scanner does not influence dose efficiency and image quality of conventional images.

In an effort to determine the influence of the dual-layer detector, a performance characterization of the DLCT was conducted at our institute and compared with the performance of a technically equivalent conventional single-layer CT scanner (SLCT; Brilliance ICT, Philips Healthcare, Best, the Netherlands), using phantoms. The SLCT was selected for this comparison, because the DLCT and SLCT are equal in terms of gantry and X-ray tube, except for a reduced titanium filtration in the DLCT compared to the SLCT to increase spectral separation between the low and high energies (i.e. increase the number of low energy photons).

\section{Materials and Methods}

\section{Scanning modes}

To study the performance of the DLCT, a quantitative comparison with the SLCT was made by comparing image quality of conventional images acquired on both scanners. The SLCT was selected, because the hardware of the DLCT and SLCT scanners is equal (apart from the detector and tube filtration). Four regular adult scanning protocols were scanned on both the DLCT and SLCT: body helical, body axial, head helical and head axial. These protocols were selected because these are used most at our institute, and because the wedge filter used on the SLCT in these protocols matches the wedge filter of the DLCT. Each protocol was scanned with tube voltages of 80, 100, 120 and $140 \mathrm{kVp}$, and with a fixed exposure of 200 mAs for the DLCT. Other scan parameters are summarized in Table 3.1. In order to reduce the likelihood of errors, all acquisitions were performed five times successively. 
Image quality of a dual-layer CT | Chapter $\mathbf{3}$

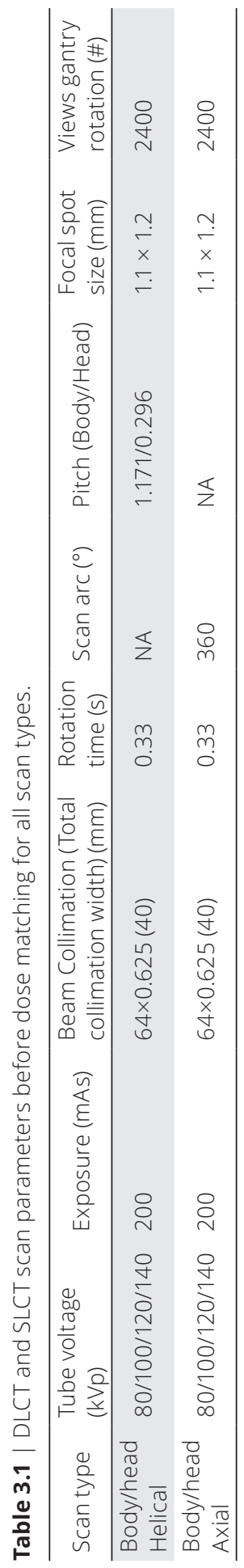


Part I | Technical aspects of dual-energy CT

\section{Dose}

There is a slight difference between the SLCT and DLCT with regard to tube filtration. To create a larger flux for the spectral separation on the DLCT, the scanner has a reduced titanium filtration. To quantify the change in spectrum resulting from the reduced filtration, the half-value layer (HVL) was measured at all tube voltages on both the SLCT and the DLCT. In order to compensate for this difference in flux, the exposure values (mAs) of the SLCT were selected such that the dose of the scanning protocols on the SLCT matched the dose of the scanning protocols of the DLCT. The volume CT dose index $\left(C T D I_{v o l}\right)$ was measured for the body and head scans using a body and head phantom (IBA Dosimetry, Bartlett, TN, USA), respectively, each with a $10 \mathrm{~cm}$ pencil ionization chamber (Raysafe, Billdal, Sweden). First, the SLCT scan parameters were set equal to the scan parameters used on the DLCT. When the difference between the CTDIs was too large, the exposure value of the protocol on the SLCT was changed to match the dose of the DLCT. A difference in CTDI up to 5\% was accepted, corresponding to an uncertainty in exposure up to $10 \mathrm{mAs}$.

\section{Image reconstruction}

Images were reconstructed using the default hybrid iterative reconstruction method iDose ${ }^{4}$ (level 1), with standard reconstruction filter for body and head ("B" and "UB", respectively) for both the SLCT and the DLCT. The iDose ${ }^{4}$ (level 1) reconstruction was chosen because it was available on both machines and it keeps the amount of postprocessing minimal. Images were reconstructed to $1 \mathrm{~mm}$ slice thickness and $1 \mathrm{~mm}$ slice spacing, using a field-of-view (FOV) of $250 \mathrm{~mm}$ and a reconstruction matrix of $512 \times 512$ pixels.

\section{Image quality}

To study the image quality of both CT scanners, a phantom study was conducted using a Catphan 600 phantom containing 5 modules (The Phantom Laboratory, Salem, NY, USA). The phantom was positioned in the isocenter of the scanner as suggested by the user manual[54]. Catphan module CTP404 was used to measure CT number linearity, spatial linearity, slice thickness and slice spacing. This module contains 7 inserts with different opacity, which have a range of approximately -1000 to 1000 Hounsfield units $(\mathrm{HU})$. Spatial linearity is verified by measuring the distances between three $3 \mathrm{~mm}$ holes with a fixed distance $(50 \mathrm{~mm})$. Slice thickness and slice spacing can be estimated using two sets of $23^{\circ}$ wire ramps, by measuring the full width at half maximum length and multiplying this with a $23^{\circ}$ ramp angle correction factor as instructed by the user manual[54]. 
Module CTP591 was used to measure spatial resolution via the modulation transfer function (MTF). The MTF was calculated using a $50 \mu \mathrm{m}$ tungsten wire source. This wire source is used to calculate a point spread function (PSF), from which the MTF was computed using a Fourier transform, and normalized to the average intensity of the PSF[55]:

$$
\operatorname{MTF}\left(f_{x}\right)=\frac{|D F T[I(x)]|}{\bar{I}} .
$$

For every acquisition, an MTF was calculated from the $x$-direction PSF and $y$-direction PSF. The wire source used for the wire source has a finite size, whereas a MTF is calculated from an infinitesimal point source. To correct for the finite size of the wire source, the following correction factor is used[56]:

$$
C F(u)=2 \frac{J_{1}(\pi u d)}{\pi u d}
$$

In which $J_{1}$ is the first order Bessel function, $u$ the spatial frequency and $d$ the diameter of the wire source. To compare the two scanners, the resolutions corresponding to 50\%, 10\% and 5\% MTF were calculated. In addition, module CTP528 was used to visually verify the spatial resolution.

Module CTP515 was used to calculate the contrast-to-noise ratio (CNR) of a 1.0\% nominal contrast target with a $15 \mathrm{~mm}$ diameter. A region of interest (ROI) was selected in the target, and a region of the same size was selected in the background. CNR was then calculated as:

$$
C N R=\frac{\bar{I}_{R O I}-\bar{I}_{b g}}{\sqrt{\frac{1}{2}\left(\sigma_{R O I}{ }^{2}+\sigma_{b g}{ }^{2}\right)}} .
$$

Here, $\bar{I}_{R O I}$ is the mean signal of the ROI, $\bar{I}_{b g}$ the mean signal of the background, $\sigma_{R O I}$ the standard deviation of the ROI and $\sigma_{b g}$ the standard deviation of the background. CNR was calculated in 5 consecutive slices in every acquisition, and from these 5 acquisitions an average CNR was calculated.

Module CTP486 is cast from a uniform material, of which the CT number (expected range: $5-18 \mathrm{HU}$ ) is designed to be within $2 \%$ of that of water. The module was used to measure the accuracy of the system by calculating a mean CT number, and to characterize noise by calculating the standard deviation (SD) of the mean CT number and by measuring the noise power spectrum (NPS). 
Nine ROIs of $64 \times 64$ voxels were selected in the uniform material, of which the SD was calculated and the 2D NPS was computed using the discrete Fourier transform[55]:

$$
N P S\left(f_{x}, f_{y}\right)=\frac{1}{R} \sum_{i=1}^{R}\left|D F T_{2 D}\left[I_{i}(x, y)-\bar{I}_{l}\right]\right|^{2} \frac{\Delta_{x} \Delta_{y}}{N_{x} N_{y}}
$$

Here, $I_{i}(x, y)$ is the signal in the i-th ROI, $\bar{I}_{\imath}$ the mean of $I_{i}(x, y)$ and $R$ the total number of ROIs. The quantities $\Delta_{x}, \Delta_{y}, N_{x}$ and $N_{y}$ are the pixel spacing and the number of pixels in the $x$ - and $y$-directions. With radial averaging the 2D NPS can be collapsed to a 1D radial frequency[55]. The mean CT number was calculated for each acquisition by calculating the mean CT number of the 9 ROls.

To study the effects of beam hardening due to patient size in body scans, an analysis of the Catphan phantom with additional attenuation was conducted. Around the Catphan phantom infusion bags filled with water were positioned, widening the diameter of the Catphan from 20 centimeters to 34 centimeters. The same setup was scanned on both the SLCT and DLCT. From these images the SD, NPS, MTF and CNR were calculated. In addition, to study the effect of very large patients in body scans, the body phantom was surrounded with water infusion bags, to create a phantom of 45 centimeters in diameter. Since such large phantoms require more penetrating radiation, they were scanned at 120 and $140 \mathrm{kVp}$ on both the SLCT and DLCT. From these images the SD and NPS was calculated.

\section{Statistical analysis}

All measurements were repeated five times to allow testing for statistical significance between the average values of the two scanners, by using an unpaired sample t-test. A significance level of 0.05 was chosen. For the comparison of the NPS, to account for multiple sampling, a Bonferroni correction was applied. The null hypothesis is that DLCT and SLCT show equal performance at equivalent dose.

\section{Results}

\section{Dose}

The HVL measurements of the SLCT showed an increase in HVL compared to the DLCT (Table 3.2).

Table 3.2 | Half-value layer measurements (mm Al) at different tube voltages.

\begin{tabular}{llllll}
\hline & \multicolumn{3}{c}{ Body scans } & & \multicolumn{2}{c}{ Head scans } \\
\cline { 2 - 3 } \cline { 6 - 6 } & DLCT & SLCT & & DLCT & SLCT \\
\hline $80 \mathrm{kVp}$ & 5.19 & 6.47 & & 5.05 & 6.56 \\
$100 \mathrm{kVp}$ & 6.86 & 8.27 & & 6.88 & 8.36 \\
$120 \mathrm{kVp}$ & 7.97 & 9.59 & & 8.13 & 9.65 \\
$140 \mathrm{kVp}$ & 9.27 & 10.8 & & 9.12 & 10.7 \\
\hline
\end{tabular}


For both body and head scans, the dose measurements showed a more than 5\% lower dose on the SLCT than on the DLCT, as can be seen in Table 3.3. Accordingly, to compensate for the dose difference, body scans on the SLCT at 80, 100, 120 and 140 $\mathrm{kVp}$ were acquired with an exposure value of 250, 240, 240 and 230 mAs respectively. Similarly, head scans on the SLCT at 80, 100, 120 and $140 \mathrm{kVp}$ were acquired with an exposure value of 250, 240, 230 and 220 mAs. Furthermore, the CTDI values as reported by the scanner are comparable to the measured CTDIs (Table 3.3).

Table 3.3 | CTDI measurements (mGy) at different tube voltages before dose matching. The CTDIs reported in the protocol are stated between brackets.

\begin{tabular}{llllllll}
\hline & \multicolumn{3}{c}{ Body scans } & & \multicolumn{3}{c}{ Head scans } \\
\cline { 2 - 4 } \cline { 8 - 9 } & DLCT & SLCT & Diff. (\%) & & DLCT & SLCT & Diff. (\%) \\
\hline $80 \mathrm{kVp}$ & $6.13(6.00)$ & $4.54(4.20)$ & 25.93 & & $11.85(11.9)$ & $9.00(8.70)$ & 24.04 \\
$100 \mathrm{kVp}$ & $11.87(11.4)$ & $9.45(8.80)$ & 20.34 & & $22.32(22.2)$ & $17.68(17.5)$ & 20.78 \\
$120 \mathrm{kVp}$ & $18.81(18.1)$ & $15.18(14.7)$ & 19.31 & & $34.16(34.3)$ & $29.18(28.7)$ & 14.58 \\
$140 \mathrm{kVp}$ & $25.91(26.0)$ & $22.00(21.6)$ & 15.09 & & $48.35(48.6)$ & $43.15(41.9)$ & 10.76 \\
\hline
\end{tabular}

Abbreviations: Diff.; difference between CTDI of DLCT and SLCT.

\section{CT number linearity}

For both scanners, the body scans showed unbiased CT number linearity. CT numbers in those scans were in accordance with expected values as stated in the user manual of the phantom (Figure 3.1). The head scans, however, showed significantly underestimated CT numbers for the high opacity inserts (Delrin: expected 344-387 HU and Teflon: expected 941-1060 HU). The underestimation was visible at all energies for the DLCT, and for the SLCT at 100, 120 and $140 \mathrm{kVp}$. For the SLCT at $80 \mathrm{kVp}$ only the Teflon insert was outside the range of expected values (Figure 3.1). Figure 3.1 is exemplary for axial scans.
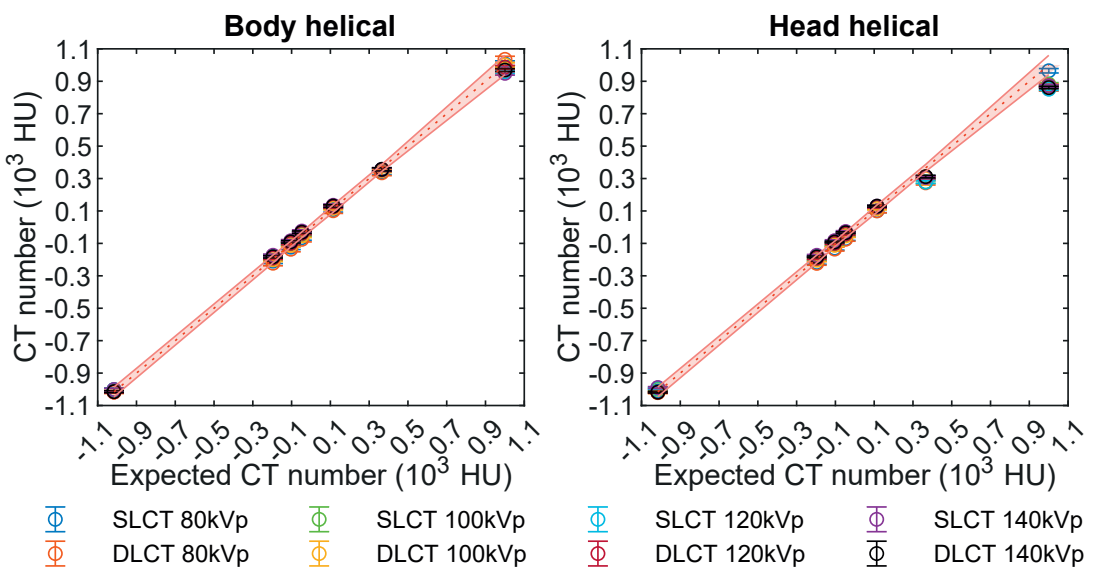

Figure 3.1 | Comparison of CT numbers (HU) at all energies of DLCT and SLCT of body helical scans and head helical scans. The range of expected values is highlighted red. 
Part I | Technical aspects of dual-energy CT

\section{Spatial linearity, slice thickness and slice spacing}

The mean distances measured to evaluate spatial linearity were all found to be within $0.1 \mathrm{~mm}$ of the expected value of $50 \mathrm{~mm}$. The differences between the SLCT and DLCT were not significant.

For both scanners, slice spacing was in accordance with the set reconstruction parameter of $1 \mathrm{~mm}$, and there is no significant difference between the scanners.

In general, the slice thickness was overestimated by both scanners, with every scan type (Table 3.4). There were no significant differences for head scans between the two scanners. For body scans, however, there was a significant difference at the 140 kVp measurements. In addition, the body axial scans at 100 and 120 kVp showed a significant difference.

Table 3.4 | Comparison of reconstructed slice thickness (mean \pm SD) (mm) for all scan types and energies. If the difference between DLCT and SLCT values is significant, both values are marked with an asterisk.

\begin{tabular}{llllll}
\hline & & $80 \mathrm{kVp}$ & $100 \mathrm{kVp}$ & $120 \mathrm{kVp}$ & $140 \mathrm{kVp}$ \\
\hline \multirow{2}{*}{ Body helical } & DLCT & $1.27 \pm 0.09$ & $1.19 \pm 0.17$ & $1.27 \pm 0.09$ & $1.39 \pm 0.09^{*}$ \\
& SLCT & $1.19 \pm 0.09$ & $1.23 \pm 0.00$ & $1.23 \pm 0.00$ & $1.23 \pm 0.00^{*}$ \\
\multirow{2}{*}{ Body axial } & DLCT & $1.35 \pm 0.11$ & $1.39 \pm 0.09^{*}$ & $1.44 \pm 0.00^{*}$ & $1.44 \pm 0.00^{*}$ \\
& SLCT & $1.27 \pm 0.09$ & $1.23 \pm 0.00^{*}$ & $1.23 \pm 0.00^{*}$ & $1.23 \pm 0.00^{*}$ \\
\multirow{2}{*}{ Head helical } & DLCT & $1.23 \pm 0.00$ & $1.23 \pm 0.00$ & $1.23 \pm 0.00$ & $1.23 \pm 0.00$ \\
& SLCT & $1.23 \pm 0.00$ & $1.23 \pm 0.00$ & $1.27 \pm 0.00$ & $1.27 \pm 0.00$ \\
Head axial & DLCT & $1.23 \pm 0.00$ & $1.23 \pm 0.00$ & $1.23 \pm 0.00$ & $1.23 \pm 0.00$ \\
& SLCT & $1.23 \pm 0.00$ & $1.35 \pm 0.11$ & $1.35 \pm 0.11$ & $1.27 \pm 0.09$ \\
\hline
\end{tabular}

Abbreviation: SD; standard deviation.

\section{Spatial resolution}

MTF curves, presented in Figure 3.2, showed a similar shape for all scan protocols on both scanners at all energies. However, the MTF curves of the SLCT appeared to vary slightly with tube voltage, whereas those of the DLCT were less dependent on tube voltage. 

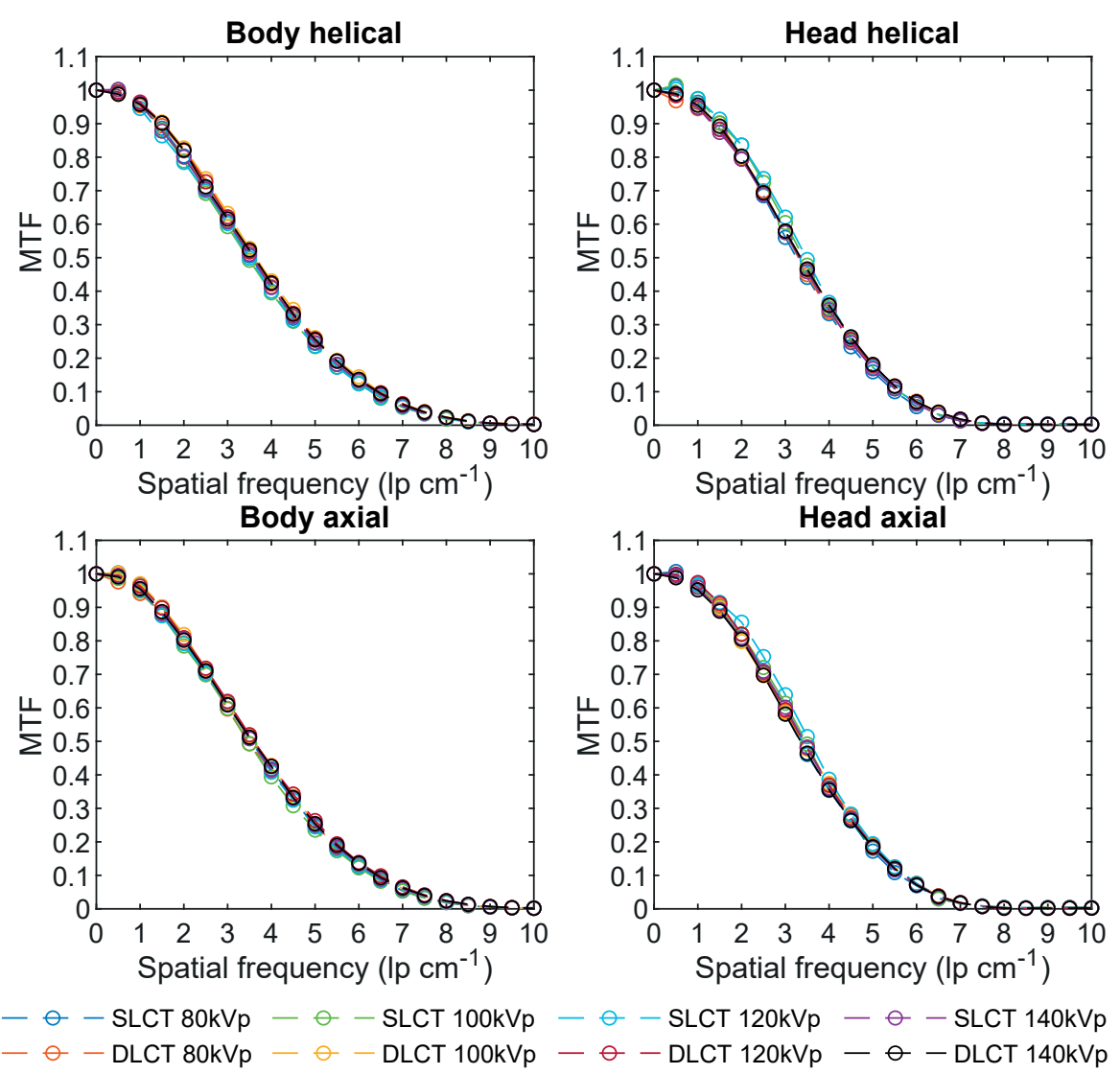

Figure 3.2 | Mean MTF curves of DLCT and SLCT for body helical, head helical, body axial, and head axial scans for all tube voltages for a phantom of $20 \mathrm{~cm}$ in diameter.

The corresponding 50\%,10\% and 5\% MTF curves showed differences in resolution between DLCT and SLCT up to 0.25 line pairs per $\mathrm{cm}(\mathrm{lp} / \mathrm{cm})$ for body scans as shown in Table 3.5 and differences up to $0.2 \mathrm{lp} / \mathrm{cm}$ for head scans, as shown in Table 3.6. The resolving power of body scans acquired on the DLCT is generally slightly higher than on the SLCT, but this difference is not always significant. Overall, the resolution of body scans was slightly higher than that of head scans. 
Part I | Technical aspects of dual-energy CT

Table 3.5 | Spatial resolutions (mean \pm SD) (Ip/cm) at 50\%, 10\% and 5\% MTF, for body scans at different energies. If the difference between the DLCT and SLCT is significant, it is marked with an asterisk.

\begin{tabular}{|c|c|c|c|c|c|c|c|}
\hline & \multirow[b]{2}{*}{ MTF } & \multicolumn{3}{|c|}{ Body helical } & \multicolumn{3}{|c|}{ Body axial } \\
\hline & & DLCT & SLCT & Diff. & $\overline{D L C T}$ & SLCT & Diff. \\
\hline \multirow{3}{*}{80 kVp } & $50 \%$ & $3.58 \pm 0.13$ & $3.53 \pm 0.11$ & 0.05 & $3.43 \pm 0.17$ & $3.57 \pm 0.17$ & -0.14 \\
\hline & $10 \%$ & $6.44 \pm 0.13$ & $6.28 \pm 0.11$ & $0.16^{*}$ & $6.48 \pm 0.19$ & $6.32 \pm 0.17$ & 0.17 \\
\hline & $5 \%$ & $7.20 \pm 0.16$ & $7.19 \pm 0.28$ & 0.01 & $7.16 \pm 0.17$ & $7.09 \pm 0.14$ & 0.07 \\
\hline \multirow{3}{*}{$100 \mathrm{kVp}$} & $50 \%$ & $3.64 \pm 0.16$ & $3.45 \pm 0.15$ & $0.18^{*}$ & $3.60 \pm 0.05$ & $3.47 \pm 0.07$ & $0.13^{*}$ \\
\hline & $10 \%$ & $6.47 \pm 0.14$ & $6.25 \pm 0.10$ & $0.22 *$ & $6.39 \pm 0.07$ & $6.26 \pm 0.08$ & $0.13^{*}$ \\
\hline & $5 \%$ & $7.27 \pm 0.18$ & $7.09 \pm 0.17$ & $0.18^{*}$ & $7.21 \pm 0.12$ & $7.07 \pm 0.10$ & $0.15^{*}$ \\
\hline \multirow{3}{*}{$120 \mathrm{kVp}$} & $50 \%$ & $3.58 \pm 0.14$ & $3.49 \pm 0.10$ & 0.09 & $3.60 \pm 0.09$ & $3.54 \pm 0.05$ & 0.06 \\
\hline & $10 \%$ & $6.44 \pm 0.19$ & $6.28 \pm 0.11$ & $0.16^{*}$ & $6.48 \pm 0.07$ & $6.32 \pm 0.09$ & $0.17 *$ \\
\hline & $5 \%$ & $7.26 \pm 0.17$ & $7.10 \pm 0.17$ & 0.16 & $7.30 \pm 0.14$ & $7.19 \pm 0.09$ & $0.11 *$ \\
\hline \multirow{3}{*}{$140 \mathrm{kVp}$} & $50 \%$ & $3.61 \pm 0.11$ & $3.55 \pm 0.06$ & 0.07 & $3.57 \pm 0.05$ & $3.54 \pm 0.05$ & 0.02 \\
\hline & $10 \%$ & $6.41 \pm 0.23$ & $6.37 \pm 0.14$ & 0.04 & $6.42 \pm 0.07$ & $6.36 \pm 0.04$ & $0.06 *$ \\
\hline & $5 \%$ & $7.22 \pm 0.25$ & $7.13 \pm 0.17$ & 0.09 & $7.26 \pm 0.11$ & $7.22 \pm 0.09$ & 0.04 \\
\hline
\end{tabular}

Abbreviations: Diff.; difference between DLCT and SLCT, SD; standard deviation.

Table 3.6 | Spatial resolutions (mean \pm SD) (lp/cm) at 50\%, 10\% and 5\% MTF, for head scans at different energies. If the difference between the DLCT and SLCT is significant, it is marked with an asterisk.

\begin{tabular}{|c|c|c|c|c|c|c|c|}
\hline & \multirow[b]{2}{*}{ MTF } & \multicolumn{3}{|c|}{ Head helical } & \multicolumn{3}{|c|}{ Head axial } \\
\hline & & DLCT & SLCT & Diff. & DLCT & SLCT & Diff. \\
\hline \multirow{3}{*}{80 kVp } & $50 \%$ & $3.29 \pm 0.14$ & $3.23 \pm 0.19$ & 0.06 & $3.42 \pm 0.11$ & $3.32 \pm 0.09$ & $0.10^{*}$ \\
\hline & $10 \%$ & $5.66 \pm 0.07$ & $5.59 \pm 0.10$ & $0.07 *$ & $5.69 \pm 0.08$ & $5.74 \pm 0.12$ & $-0.05^{\star}$ \\
\hline & $5 \%$ & $6.26 \pm 0.08$ & $6.08 \pm 0.13$ & $0.18 *$ & $6.31 \pm 0.06$ & $6.20 \pm 0.09$ & $0.11^{*}$ \\
\hline \multirow{3}{*}{100 kVp } & $50 \%$ & $3.32 \pm 0.14$ & $3.41 \pm 0.12$ & -0.09 & $3.39 \pm 0.11$ & $3.47 \pm 0.04$ & $-0.08 *$ \\
\hline & $10 \%$ & $5.65 \pm 0.07$ & $5.57 \pm 0.10$ & 0.08 & $5.72 \pm 0.05$ & $5.71 \pm 0.06$ & 0.01 \\
\hline & $5 \%$ & $6.27 \pm 0.08$ & $6.17 \pm 0.06$ & $0.10 *$ & $6.32 \pm 0.05$ & $6.31 \pm 0.04$ & $0.09 *$ \\
\hline \multirow{3}{*}{120 kVp } & $50 \%$ & $3.35 \pm 0.09$ & $3.47 \pm 0.09$ & $-0.13 *$ & $3.42 \pm 0.06$ & $3.55 \pm 0.02$ & $-0.14 *$ \\
\hline & $10 \%$ & $5.66 \pm 0.04$ & $5.59 \pm 0.07$ & $0.07^{\star}$ & $5.69 \pm 0.03$ & $5.74 \pm 0.05$ & $-0.05^{*}$ \\
\hline & $5 \%$ & $6.30 \pm 0.05$ & $6.18 \pm 0.04$ & $0.12^{\star}$ & $6.30 \pm 0.04$ & $6.31 \pm 0.04$ & -0.01 \\
\hline \multirow{3}{*}{140 kVp } & $50 \%$ & $3.35 \pm 0.10$ & $3.32 \pm 0.05$ & 0.03 & $3.35 \pm 0.05$ & $3.43 \pm 0.06$ & $-0.07 *$ \\
\hline & $10 \%$ & $5.65 \pm 0.04$ & $5.58 \pm 0.07$ & $0.07 *$ & $5.68 \pm 0.03$ & $5.67 \pm 0.03$ & 0.01 \\
\hline & $5 \%$ & $6.29 \pm 0.05$ & $6.16 \pm 0.05$ & $0.13^{*}$ & $6.28 \pm 0.04$ & $6.25 \pm 0.03$ & 0.03 \\
\hline
\end{tabular}

Abbreviations: Diff.; difference between DLCT and SLCT, SD; standard deviation.

In Figure 3.3, MTF curves of the 34 centimeter Catphan phantom, showed an increase in variation between the different tube voltages for both scanners as compared to Figure 3.2. The shape and magnitude, however, was very similar compared to Figure 3.2. For body scans a spatial resolution of more than $7 \mathrm{lp} / \mathrm{cm}$ was found (5\% MTF). These values are comparable to the earlier results in Figure 3.2. 

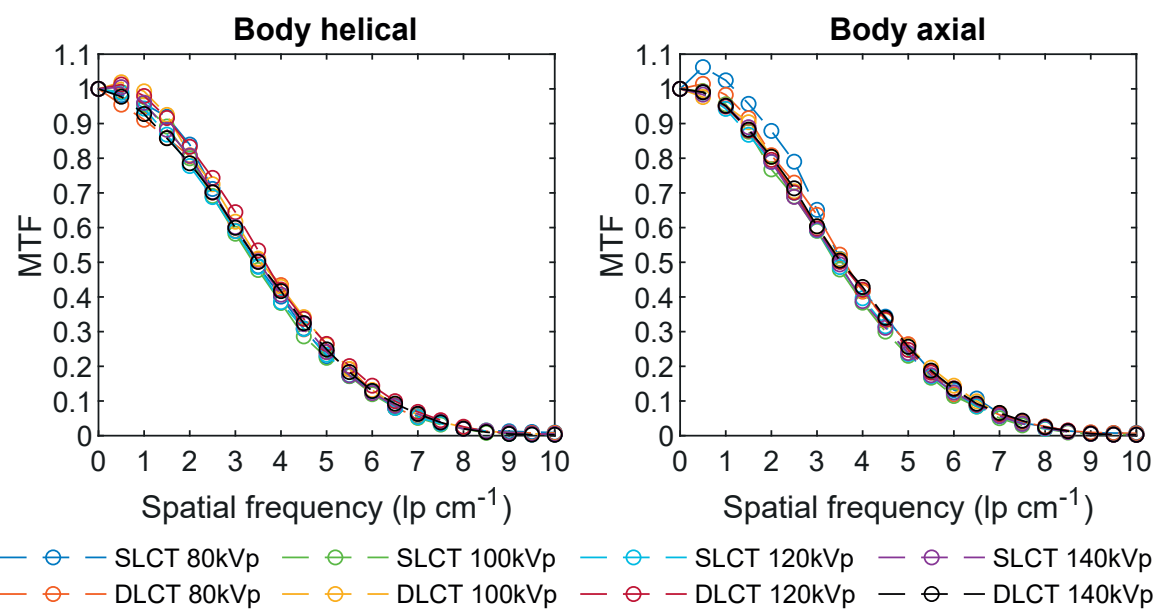

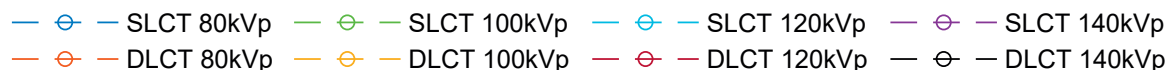

Figure 3.3 | Mean MTF curves of DLCT and SLCT for body helical and body axial scans for all tube voltages for a phantom of $34 \mathrm{~cm}$ in diameter.

\section{Visual inspection of spatial resolution}

To verify the above measurements, images of the high resolution module in the Catphan 600 phantom were visually inspected. In general, the high resolution bars are resolved slightly better on the DLCT than on the SLCT. In Figure 3.4 some of these images are presented. These images demonstrate that the bars are clearly separable at $7 \mathrm{lp} / \mathrm{cm}$, but start to blur at $8 \mathrm{lp} / \mathrm{cm}$ for body scans on both the DLCT and the SLCT. However, the bars on the DLCT are somewhat more visible than on the SLCT. This confirms the observation that at 5\% MTF the resolution is higher than $7 \mathrm{lp} / \mathrm{cm}$. In a similar fashion, head scans acquired on the DLCT and the SLCT clearly separate the bars at $6 \mathrm{lp} / \mathrm{cm}$, but start to blur at $7 \mathrm{lp} / \mathrm{cm}$, confirming the observation at 5\% MTF. 

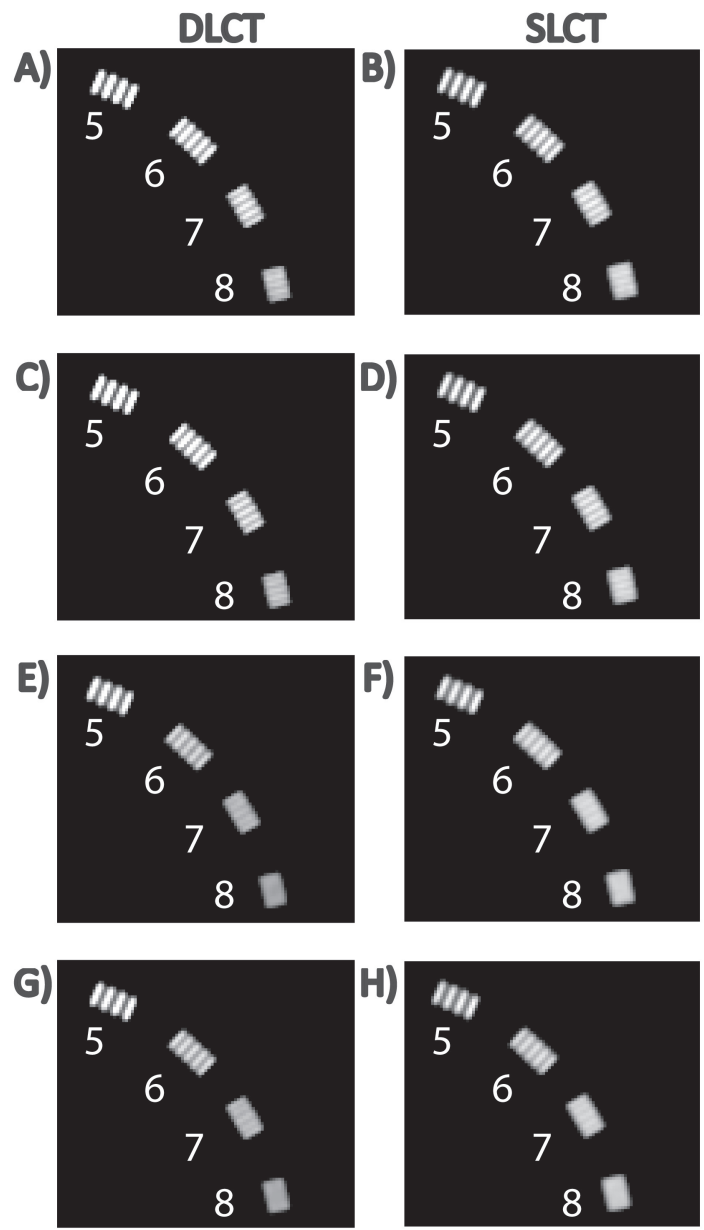

Figure 3.4 | High contrast resolution bars for body helical (A, B), body axial (C, D), head helical $(E, F)$, and head axial $(G, H)$ for DLCT and SLCT at $140 \mathrm{kVp}$. These images are exemplary for the other tube voltages.

\section{Noise characterization}

The noise (SD) of the different scanning protocols is shown in Table 3.7. For most combinations of scan types and tube voltages, the noise of the DLCT was higher, but in some instances, especially for head helical scans, the noise of the SLCT was higher. 
Table 3.7 | Noise levels (mean \pm SD) for all scan types at different energies for a phantom of 20 centimeters in diameter. If the difference (\%) between DLCT and SLCT is significant, it is marked with an asterisk.

\begin{tabular}{llllll}
\hline & & $80 \mathrm{kVp}$ & $100 \mathrm{kVp}$ & $120 \mathrm{kVp}$ & $140 \mathrm{kVp}$ \\
\hline \multirow{3}{*}{ Body helical } & DLCT & $15.31 \pm 0.09$ & $10.72 \pm 0.07$ & $8.48 \pm 0.06$ & $7.24 \pm 0.11$ \\
& SLCT & $14.83 \pm 0.15$ & $10.37 \pm 0.10$ & $8.11 \pm 0.04$ & $7.46 \pm 0.08$ \\
& Diff. & $3.19^{*}$ & $3.30^{*}$ & $4.36^{*}$ & $-3.00^{*}$ \\
& DLCT & $13.44 \pm 0.13$ & $9.24 \pm 0.05$ & $7.17 \pm 0.03$ & $6.04 \pm 0.05$ \\
Body axial & SLCT & $12.72 \pm 0.08$ & $8.93 \pm 0.07$ & $6.95 \pm 0.08$ & $5.97 \pm 0.02$ \\
& Diff. & $5.33^{*}$ & $3.41^{*}$ & $3.11^{*}$ & 1.32 \\
& DLCT & $11.69 \pm 0.11$ & $8.12 \pm 0.03$ & $6.32 \pm 0.06$ & $5.33 \pm 0.03$ \\
Head helical & SLCT & $12.33 \pm 0.13$ & $8.92 \pm 0.07$ & $7.38 \pm 0.04$ & $6.71 \pm 0.08$ \\
& Diff. & $-5.46^{*}$ & $-9.86^{*}$ & $-16.80^{*}$ & $-25.81^{*}$ \\
& DLCT & $11.39 \pm 0.08$ & $7.88 \pm 0.07$ & $6.03 \pm 0.04$ & $5.11 \pm 0.02$ \\
Head axial & SLCT & $11.03 \pm 0.10$ & $7.65 \pm 0.03$ & $6.03 \pm 0.07$ & $5.17 \pm 0.03$ \\
& Diff. & $3.14^{*}$ & $2.97^{*}$ & -0.06 & $-1.18^{*}$ \\
\hline
\end{tabular}

Abbreviations: Diff;; difference between DLCT and SLCT, SD; standard deviation.

In Figure 3.5, the NPS curves are shown. The NPS curves of both scanners were generally similar in shape, but the curves with high tube voltage sometimes show a low frequency peak. The DLCT illustrated slightly higher noise levels compared to the SLCT, this difference was significant for the head helical scans at $80 \mathrm{kVp}$ and scans at $120 \mathrm{kVp}$ (except for head axial scans). This significant difference is mainly found in the low frequency range of the NPS (i.e. $0.16 \mathrm{~mm}^{-1}$ to $0.64 \mathrm{~mm}^{-1}$ ). In addition, for body helical scans at $140 \mathrm{kVp}$ the tails of the NPS (i.e. $>0.64 \mathrm{~mm}^{-1}$ ) were significantly different. 

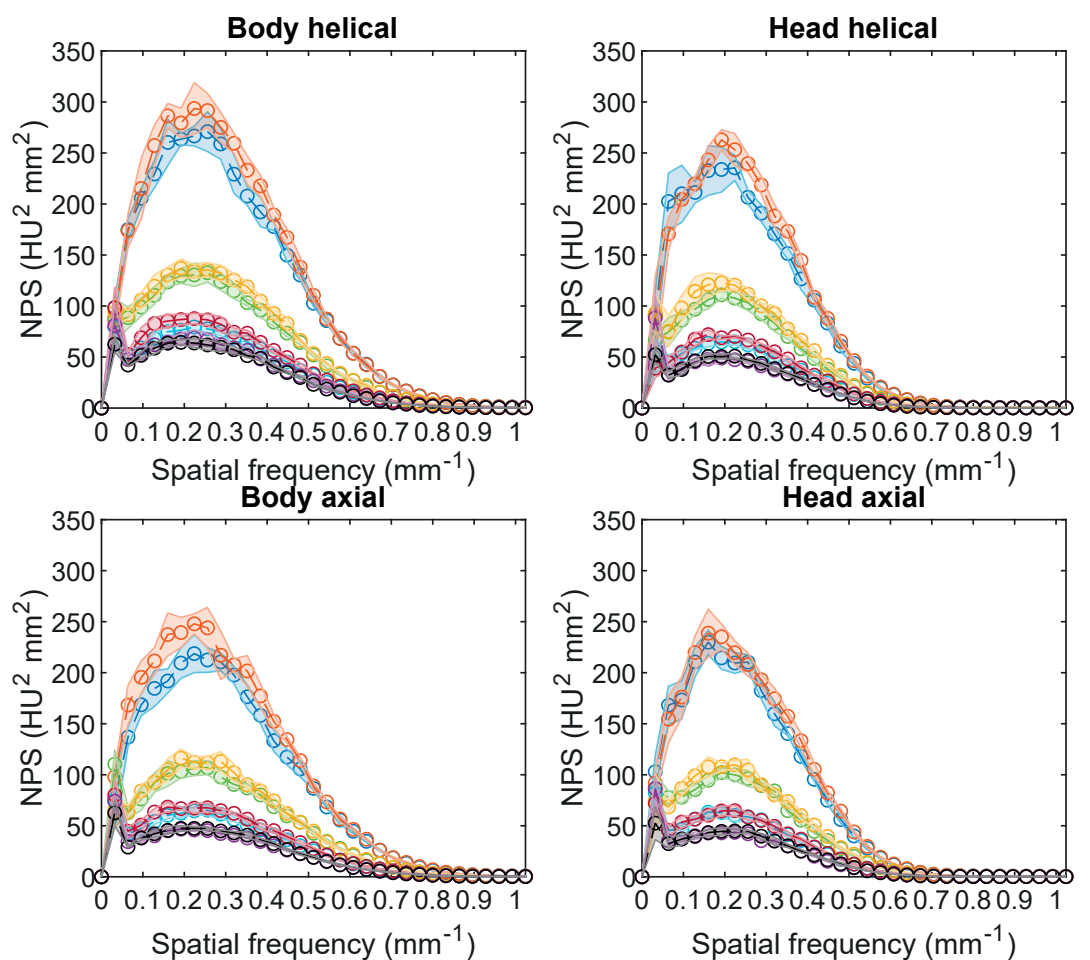

$-\ominus-$ SLCT 80kVp - $\ominus-$ SLCT 100kVp - $\ominus-$ SLCT 120kVp - $\ominus-$ SLCT 140kVp

$-\ominus-$ DLCT $80 \mathrm{kVp}-\ominus-$ DLCT 100kVp - $\ominus-$ DLCT 120kVp - $\ominus-$ DLCT 140kVp

Figure 3.5 | Comparison of NPS curves for a phantom of $20 \mathrm{~cm}$ in diameter between DLCT and SLCT at different energies, for body helical, head helical, body axial, and head axial. Mean values with 95\% confidence intervals are shown.

For the 34 centimeter phantom, an increase in noise (SD) (Table 3.8) is observed as compared to the 20 centimeter Catphan phantom (Table 3.7). The DLCT had a significant higher noise level as compared to the SLCT for all tube voltages, except for body helical scans at $140 \mathrm{kVp}$.

Table 3.8 | Noise levels (mean \pm SD) for body scans at different energies for a phantom of 34 centimeters in diameter. If the difference (\%) between DLCT and SLCT is significant, it is marked with an asterisk.

\begin{tabular}{llllll}
\hline & & $80 \mathrm{kVp}$ & $100 \mathrm{kVp}$ & $120 \mathrm{kVp}$ & $140 \mathrm{kVp}$ \\
\hline \multirow{3}{*}{ Body helical } & SLCT & $17.59 \pm 0.19$ & $12.30 \pm 0.24$ & $9.70 \pm 0.12$ & $8.13 \pm 0.07$ \\
& DLCT & $17.03 \pm 0.29$ & $11.53 \pm 0.12$ & $9.04 \pm 0.16$ & $8.17 \pm 0.25$ \\
& Diff. & $3.17^{*}$ & $6.23^{*}$ & $6.77^{*}$ & -0.51 \\
& DLCT & $15.59 \pm 0.09$ & $10.63 \pm 0.07$ & $8.28 \pm 0.06$ & $6.99 \pm 0.05$ \\
Body axial & SLCT & $14.45 \pm 0.08$ & $10.13 \pm 0.07$ & $7.89 \pm 0.03$ & $6.76 \pm 0.01$ \\
& Diff. & $7.34^{*}$ & $4.73^{*}$ & $4.69 *$ & $3.29 *$ \\
\hline
\end{tabular}

Abbreviations: Diff.; difference between DLCT and SLCT, SD; standard deviation. 
In Figure 3.6, the NPS curves of the 34 centimeter phantom are shown. These curves show that for body scans at $140 \mathrm{kVp}$ there is no difference between SLCT and DLCT. At 80, 100 and $120 \mathrm{kVp}$, the DLCT has a significant higher noise level for axial body scans. For 100 and $120 \mathrm{kVp}$ these differences are found in the tail of the NPS (i.e. > $0.64 \mathrm{~mm}^{-1}$ ), whereas at $80 \mathrm{kVp}$ over the entire range of the NPS (i.e. $0.16 \mathrm{~mm}^{-1}$ to 1.02 $\mathrm{mm}^{-1}$ ) differences are found.
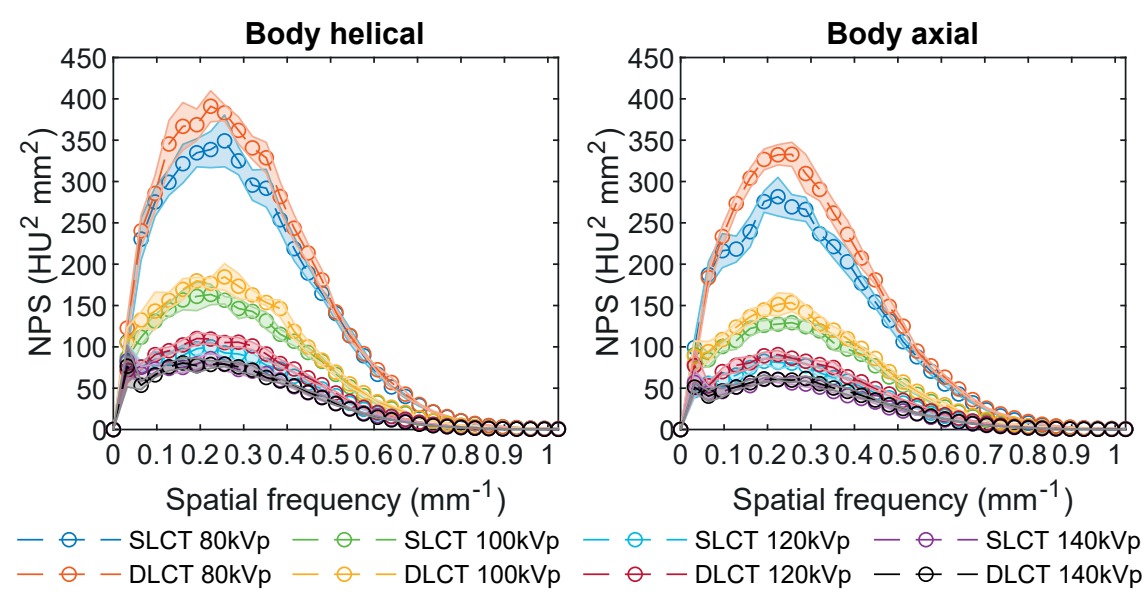

Figure 3.6 | Comparison of NPS curves for a phantom of $34 \mathrm{~cm}$ in diameter between DLCT and SLCT at different energies, for body helical and body axial scans. Mean values with $95 \%$ confidence intervals are shown.

In Table 3.9, noise levels (SD) for body scans for the 45 centimeter phantom are given. A significant difference is observed for all tube voltages. This difference is in favor of the SLCT.

Table 3.9 | Noise levels (mean \pm SD) for body scans at 120 and $140 \mathrm{kVp}$ for a phantom of 45 centimeters in diameter. If the difference (\%) between DLCT and SLCT is significant, it is marked with an asterisk.

\begin{tabular}{llll}
\hline & & $120 \mathrm{kVp}$ & $140 \mathrm{kVp}$ \\
\hline \multirow{3}{*}{ Body helical } & DLCT & $69.07 \pm 1.34$ & $53.84 \pm 0.49$ \\
& SLCT & $61.10 \pm 0.83$ & $53.04 \pm 0.63$ \\
& Diff. & $11.54^{*}$ & $1.48^{*}$ \\
\multirow{3}{*}{ Body axial } & DLCT & $57.86 \pm 0.37$ & $46.56 \pm 0.18$ \\
& SLCT & $51.08 \pm 0.32$ & $44.36 \pm 0.26$ \\
& Diff. & $11.71^{*}$ & $4.74^{*}$ \\
\hline
\end{tabular}

Abbreviations: Diff.; difference between DLCT and SLCT, SD; standard deviation. 
The accompanying NPS curves are shown in Figure 3.7. These curves show a similar shape at all tube voltages. The differences between the SLCT and DLCT were significant for all tube voltages except for body axial scans at $140 \mathrm{kVp}$. The differences for body helical scan were mainly observed in the tail of the NPS (i.e. $>0.64 \mathrm{~mm}^{-1}$ ), whereas for the body axial scans at $120 \mathrm{kVp}$ over the entire range of the NPS (i.e. $0.16 \mathrm{~mm}-1$ to 1.02 $\mathrm{mm}^{-1}$ ). In comparison to the NPS of the smaller phantoms (Figure 3.5 and Figure 3.6), a shift in peak frequency was observed.
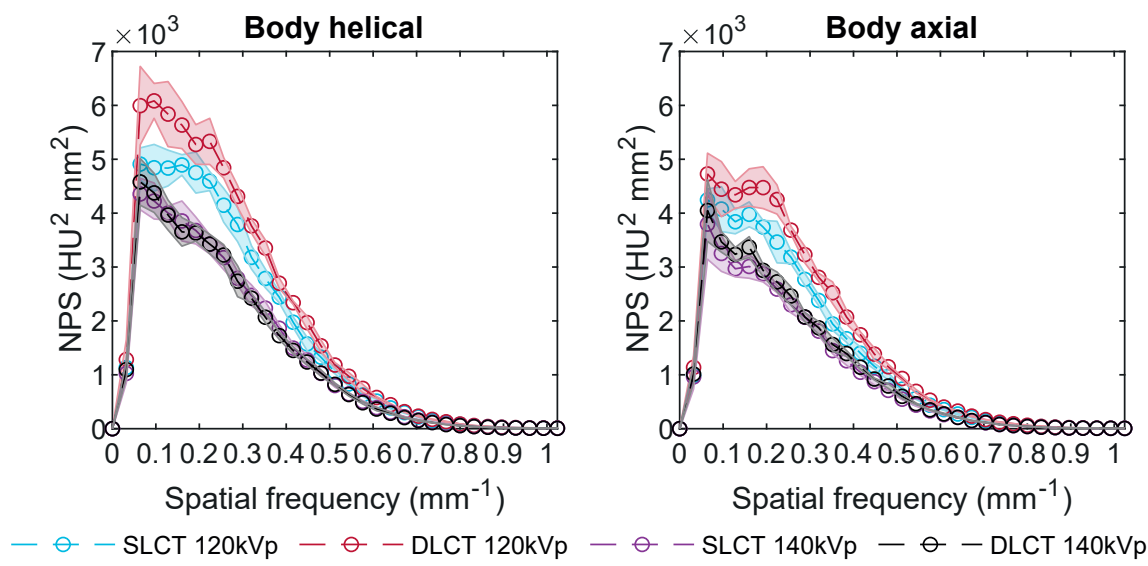

Figure 3.7 | Comparison of NPS curves for a phantom of $45 \mathrm{~cm}$ in diameter between DLCT and SLCT at 120 and $140 \mathrm{kVp}$, for body helical and body axial scans. Mean values with 95\% confidence intervals are shown.

\section{CNR}

The mean CNR of the DLCT was generally slightly higher than that of the SLCT, but the difference was not always significant, as can be seen in Table 3.10. Differences up to 0.35 are seen, where the difference for head scans from DLCT and SLCT was significant at all tube voltages, except for head scans at $100 \mathrm{kVp}$, whereas for body scans the difference was not.

Table 3.10 | CNR (mean \pm SD) for all scan types at all energies. If the difference between DLCT and SLCT is significant, both CNR values are marked with an asterisk.

\begin{tabular}{llllll}
\hline & & $80 \mathrm{kVp}$ & $100 \mathrm{kVp}$ & $120 \mathrm{kVp}$ & $140 \mathrm{kVp}$ \\
\hline \multirow{2}{*}{ Body helical } & DLCT & $0.65 \pm 0.21$ & $0.92 \pm 0.18^{*}$ & $1.21 \pm 0.21$ & $1.51 \pm 0.26$ \\
& SLCT & $0.63 \pm 0.23$ & $1.07 \pm 0.24^{*}$ & $1.33 \pm 0.25$ & $1.42 \pm 0.28$ \\
\multirow{3}{*}{ Body axial } & DLCT & $0.83 \pm 0.19$ & $1.09 \pm 0.19$ & $1.54 \pm 0.21^{*}$ & $1.76 \pm 0.26^{*}$ \\
& SLCT & $0.77 \pm 0.20$ & $1.10 \pm 0.17$ & $1.34 \pm 0.23^{*}$ & $1.60 \pm 0.22^{*}$ \\
\multirow{2}{*}{ Head helical } & DLCT & $0.92 \pm 0.27^{*}$ & $1.35 \pm 0.24$ & $1.62 \pm 0.25^{*}$ & $2.03 \pm 0.34^{*}$ \\
& SLCT & $0.73 \pm 0.24^{*}$ & $1.25 \pm 0.22$ & $1.42 \pm 0.27^{*}$ & $1.74 \pm 0.30^{*}$ \\
Head axial & DLCT & $0.91 \pm 0.24^{*}$ & $1.33 \pm 0.21$ & $1.81 \pm 0.23^{*}$ & $2.08 \pm 0.24^{*}$ \\
& SLCT & $0.68 \pm 0.23^{*}$ & $1.22 \pm 0.27$ & $1.53 \pm 0.28^{*}$ & $1.82 \pm 0.34^{*}$ \\
\hline
\end{tabular}

Abbreviations: SD; standard deviation. 
For the 34 centimeter phantom, the mean CNR of the DLCT was generally higher than that of the SLCT, as can be seen in Table 3.11. The differences were significant for all body helical scans, and for two of the body axial scans, including the only case where the CNR of the SLCT was larger than that of the DLCT (at $80 \mathrm{kVp}$ ). A clear decrease in CNR can be observed by comparing Table 3.10 with Table 3.11.

Table 3.11 | CNR (mean \pm SD) for body scans at all energies for a phantom of 34 centimeters in diameter. If the difference between DLCT and SLCT is significant, both CNR values are marked with an asterisk.

\begin{tabular}{llllll}
\hline & & $80 \mathrm{kVp}$ & $100 \mathrm{kVp}$ & $120 \mathrm{kVp}$ & $140 \mathrm{kVp}$ \\
\hline Body helical & DLCT & $0.38 \pm 0.21^{\star}$ & $0.68 \pm 0.21^{*}$ & $0.75 \pm 0.17^{\star}$ & $1.05 \pm 0.20^{*}$ \\
& SLCT & $0.21 \pm 0.23^{*}$ & $0.37 \pm 0.19^{*}$ & $0.41 \pm 0.15^{*}$ & $0.54 \pm 0.26^{*}$ \\
Body axial & DLCT & $0.32 \pm 0.16^{*}$ & $0.76 \pm 0.18$ & $0.94 \pm 0.24$ & $1.12 \pm 0.22^{*}$ \\
& SLCT & $0.41 \pm 0.13^{*}$ & $0.73 \pm 0.22$ & $0.84 \pm 0.14$ & $0.99 \pm 0.20^{*}$ \\
\hline
\end{tabular}

Abbreviations: SD; standard deviation.

\section{Mean CT numbers}

In Table 3.12, the mean CT numbers are presented. The CT numbers calculated for the scans acquired at $100 \mathrm{kVp}$ for the SLCT, and at $120 \mathrm{kVp}$ for both scanners and $140 \mathrm{kVp}$ for the DLCT were within the expected values of the phantom for both scanners. Scans acquired at $80 \mathrm{kVp}$ on both scanners, scans acquired at $100 \mathrm{kVp}$ on the DLCT and scans acquired at $140 \mathrm{kVp}$ on the SLCT are not within the expected range of 5-18 HU, but are within $2 \%$ of the CT numbers of water (-25 - $25 \mathrm{HU}$ ). The difference in CT numbers between the SLCT and the DLCT is significant for all scan types at all tube voltages.

Table 3.12 | Mean CT number (mean \pm SD) (HU) of uniform material, which by design has CT numbers within $2 \%$ of the $\mathrm{HU}$ of water $(-25-25 \mathrm{HU})$, with an expected range of 5-18 HU.

\begin{tabular}{llllll}
\hline & & $80 \mathrm{kVp}$ & $100 \mathrm{kVp}$ & $120 \mathrm{kVp}$ & $140 \mathrm{kVp}$ \\
\hline Body helical & DLCT & $-19.65 \pm 15.36$ & $-0.47 \pm 10.74$ & $9.10 \pm 8.48$ & $15.69 \pm 7.20$ \\
& SLCT & $-9.07 \pm 14.83$ & $7.29 \pm 10.38$ & $15.61 \pm 8.09$ & $20.87 \pm 7.48$ \\
Body axial & DLCT & $-18.44 \pm 13.45$ & $0.26 \pm 9.25$ & $9.27 \pm 7.18$ & $15.52 \pm 6.03$ \\
& SLCT & $-9.36 \pm 12.71$ & $7.01 \pm 8.92$ & $15.29 \pm 6.97$ & $20.25 \pm 5.97$ \\
Head helical & DLCT & $-17.83 \pm 11.71$ & $-0.99 \pm 8.12$ & $8.56 \pm 6.30$ & $14.97 \pm 5.34$ \\
& SLCT & $-13.04 \pm 12.26$ & $6.54 \pm 8.94$ & $15.94 \pm 7.41$ & $20.36 \pm 6.70$ \\
Head axial & DLCT & $-17.15 \pm 11.34$ & $-0.56 \pm 7.86$ & $8.77 \pm 6.05$ & $14.79 \pm 5.11$ \\
& SLCT & $-10.21 \pm 11.05$ & $8.64 \pm 7.67$ & $17.69 \pm 6.02$ & $21.58 \pm 5.15$ \\
\hline
\end{tabular}

Abbreviations: SD; standard deviation.

\section{Visual impression of images}

Although an observer study was beyond the scope of this study, a visual impression of the acquired images is shown in Figure 3.8. The images acquired on the SLCT and the DLCT appear very similar with regard to noise level, contrast and resolution. 
Part I | Technical aspects of dual-energy CT

The images on the SLCT have a different window level, because we have to compensate for the small difference in recorded CT numbers between SLCT and DLCT. This difference in CT numbers was already observed in Table 3.12

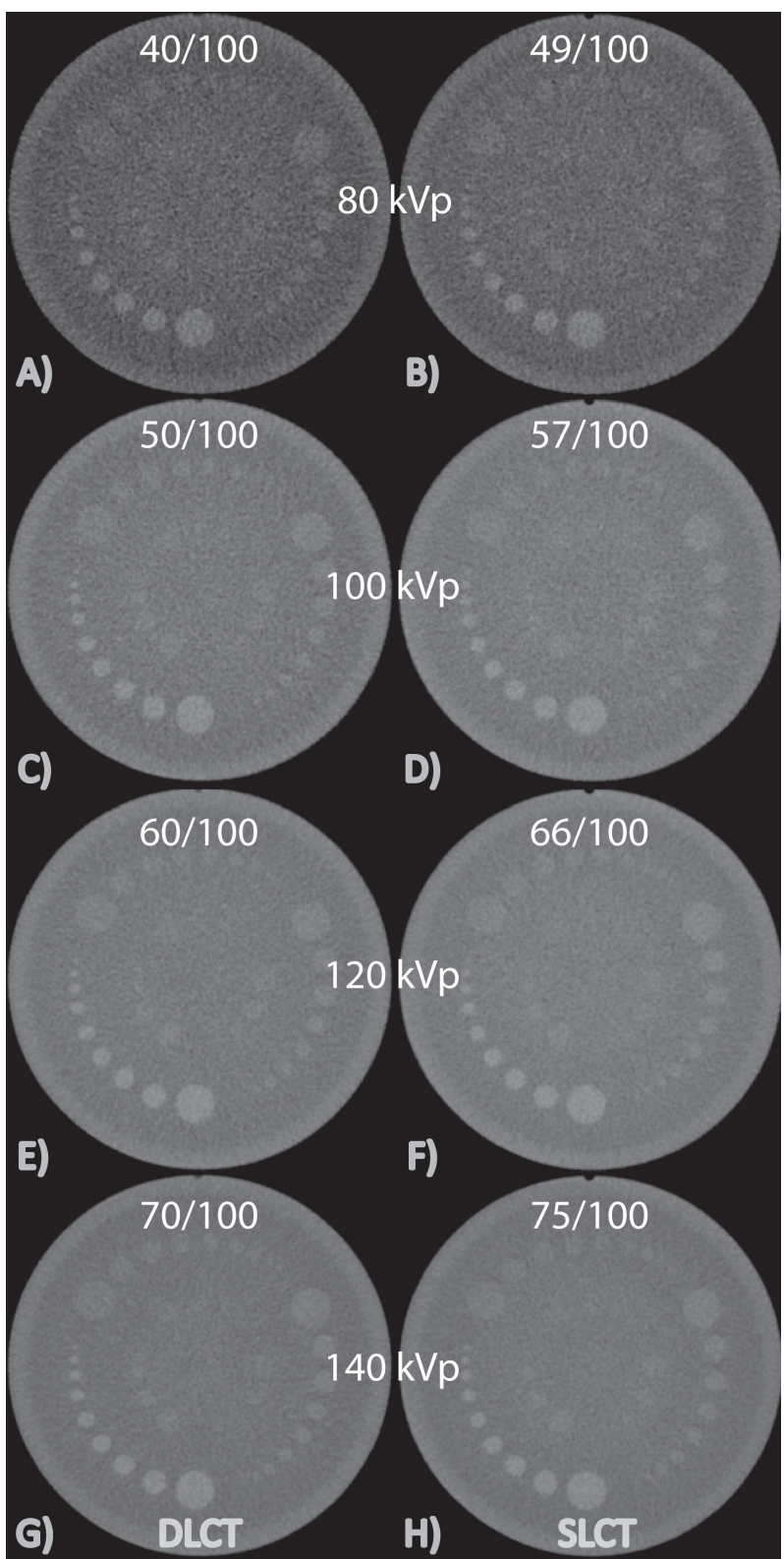

Figure 3.8 | Impression of images acquired at $80 \mathrm{kVp}(\mathrm{A}, \mathrm{B}), 100 \mathrm{kVp}(\mathrm{C}, \mathrm{D}), 120 \mathrm{kVp}(\mathrm{E}, \mathrm{F})$, and 140 $\mathrm{kVp}(\mathrm{G}, \mathrm{H})$ with DLCT and SLCT; here, axial body scans are presented. The used ratio of window level and width (HU) is given in the images. 


\section{Discussion}

In this study, the performance of the DLCT and SLCT with respect to phantom image quality of conventional images was evaluated quantitatively and qualitatively at equal dose. A number of studies have been conducted on the dual-layer technology, primarily looking at applications of dual-energy analysis[30,40,42-44,53,57,58]. However, only few studies have yet been published on image quality of conventional images acquired on the DLCT[41,59]. Ozguner et al. (2016) studied the performance of DECT acquisition on the DLCT by comparing image quality of monoenergetic and conventional images both acquired on the DLCT. Hojjati et al. (2017) evaluated conventional (diagnostic) images from the DLCT and from a conventional single-layer detector scanner, both in a phantom and in abdominal clinical images to see whether the image quality and other metrics meet the requirements of ACR guidelines. But the influence of the dual-layer detector on image quality and dose of conventional images has not been established yet.

In general, the conventional images acquired on the DLCT are very similar to images acquired on the SLCT. Body scans acquired on the DLCT, however, demonstrated a slightly increased resolving power at each tube voltage compared with the SLCT. For body scans the mean MTFs at 50\%, 10\% and 5\% are slightly higher, albeit not always significantly. The high resolution bars confirm the above observations that for all body scans on DLCT and SLCT bars separated by at least $7 \mathrm{lp} / \mathrm{cm}$ are clearly visible. In the study by Hojjati et al.[41], the resolving power for an adult body protocol was found to be $8 \mathrm{lp} / \mathrm{cm}$ for both DLCT and SLCT scanners. However, the authors did not mention the used settings for reconstruction kernel, FOV, and reconstruction matrix, which all influence spatial resolution. Ozguner et al.[59] demonstrated a resolving power of 7 $\mathrm{Ip} / \mathrm{cm}$, also for an adult body scan, similar to the resolution found in our study. Head scans also did not always show a significant difference between the SLCT and the DLCT, as is confirmed visually by the high resolution bars. The visual spatial resolution was found to be at least $6 \mathrm{lp} / \mathrm{cm}$. The 34 centimeter phantom caused more variation in the MTF curves between the different tube voltages. However, the resolving power of body scans was similar to that of the normal 20 centimeter Catphan phantom, i.e. more than $7 \mathrm{lp} / \mathrm{cm}$ for both scanners.

On both scanners a shift in peak frequency was observed between the NPS measurements in the 20 and 34 centimeter phantoms and those in the 45 centimeter phantom. One explanation is that the non-linear nature of iDose $\mathrm{m}^{4}$ ight start to play a role and affects spatial resolution and therefore noise texture. Another explanation might be an increase in scattering, resulting in an increase in low frequency noise. 
The overall noise levels (SD) of head scans on the DLCT are generally lower than those of the SLCT, and differences were significant for most tube voltages. For body scans on the other hand, the overall noise levels are generally higher on the DLCT compared to the SLCT. The differences in noise of body scans are significant for almost all tube voltages for all phantom sizes. This suggests a slightly reduced dose efficiency of the DLCT for these scans compared to the SLCT. This might be due to the softer beam, resulting from the reduced filtration on the DLCT. Altogether, noise levels differed between both scanners, with difference values depending on the scan situation. These noise level differences are generally significant. Yet, most observed differences are within the $15 \%$ typical variation of noise performance specified for both scanners by the vendor[60].

An important parameter regarding image quality is CNR. CNR was studied using a 1\% nominal contrast target. In general, CNR was higher for the DLCT than for the SLCT. The difference, however, was not always significant. The difference can be attributed mainly to the fact that on the DLCT a larger contrast between the ROI and the background was observed. This larger contrast on the DLCT can be attributed mainly to the difference in mean energy of the spectra of the two scanners. The attenuation of materials is energy-dependent, and hence contrast is energy dependent. With decreasing mean energy, contrast increases. The reduced filtration on the DLCT causes a decrease in the mean energy of the spectrum, and causes an increase in contrast as compared with the SLCT. The introduction of additional attenuation, i.e. additional noise, results in a decrease in CNR.

A reason for the difference observed in mean CT numbers between the SLCT and DLCT could be an increase in beam-hardening for the DLCT compared with the SLCT. The DLCT has a reduced filtration, which results in an increase in low energy photons. These photons are more likely to be attenuated in the patient, which results in beamhardening of the flux of photons. Beam-hardening is a phenomenon known for causing underestimation of CT numbers[61].

This study has several limitations. First, the hybrid iterative reconstruction method iDose $^{4}$ was used. We are aware that by using this reconstruction method, image quality metrics are affected[62-64]. To illustrate these effects, we compared images

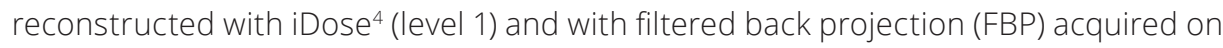
the SLCT. This comparison showed that only the magnitude of the noise and therefore the CNR was affected by the use of this reconstruction method. This was supported by the study by Löve et al.[62]. The magnitude of the NPS increased by 1.23 for body scans and 1.20 for head scans with using FBP compared to iDose 4 . All other metrics were not affected by the use of iDose ${ }^{4}$ (level 1) compared to FBP. Which means that

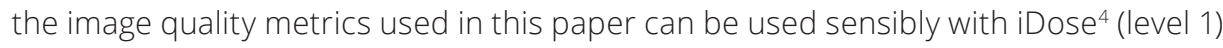
reconstructed images. 
Secondly, the MTF and CNR should be used with care in case of non-linear reconstruction methods such as iDose ${ }^{4}$. Yet, for the purpose of this comparative study, in which the same iterative reconstruction settings are used, these metrics improve comprehensibility and clarity. Moreover, these metrics have been widely used in multiple other comparative studies using iterative reconstruction methods[62,65,66]. Thirdly, we studied the image quality of the DLCT using a fixed set of scan and reconstruction parameters, whereas other settings, for instance collimation, reconstructed slice thickness, and reconstruction kernel also have an effect on image quality[67]. Our rationale was to select the scan and reconstruction parameters that are used most at our institute and moreover are used commonly for quality control assessment.

In conclusion, the results of this work indicate that the performance of the DLCT is nearly identical to the performance of the SLCT in terms of CT number linearity, spatial linearity, slice thickness, and spacing for all phantom sizes. The mean CT number of the DLCT was significantly different from that of the SLCT. While in terms of noise the SLCT performed marginally better, values of contrast and resolution where slightly in favor of the DLCT. Since these measures are exchangeable to some degree, we can conclude that for medium-sized $(20 \mathrm{~cm})$ phantoms both scanners have similar performance. For larger phantoms (34 and $45 \mathrm{~cm}$ ) at $80-120 \mathrm{kVp}$, dose efficiency is degraded for the DLCT as compared to the SLCT. This suggests that the introduction of a dual-layer detector does not compromise image quality of conventional images for normal-sized patients, but slightly degrades dose efficiency for large patients at lower tube voltages. 



\title{
CHAPTER 4
}

\section{IMAGE QUALITY OF VIRTUAL MONOCHROMATIC IMAGES IN NON-CONTRAST BRAIN CT}

\begin{abstract}
Based on
F van Ommen, F Kauw, E Bennink, JJ Heit, DN Wolman, JW Dankbaar, HWAM de Jong and M Wintermark (2020). Image quality of virtual monochromatic reconstructions of non-contrast CT on a dual-source CT scanner in adult patients. Academic Radiology, in press.
\end{abstract}

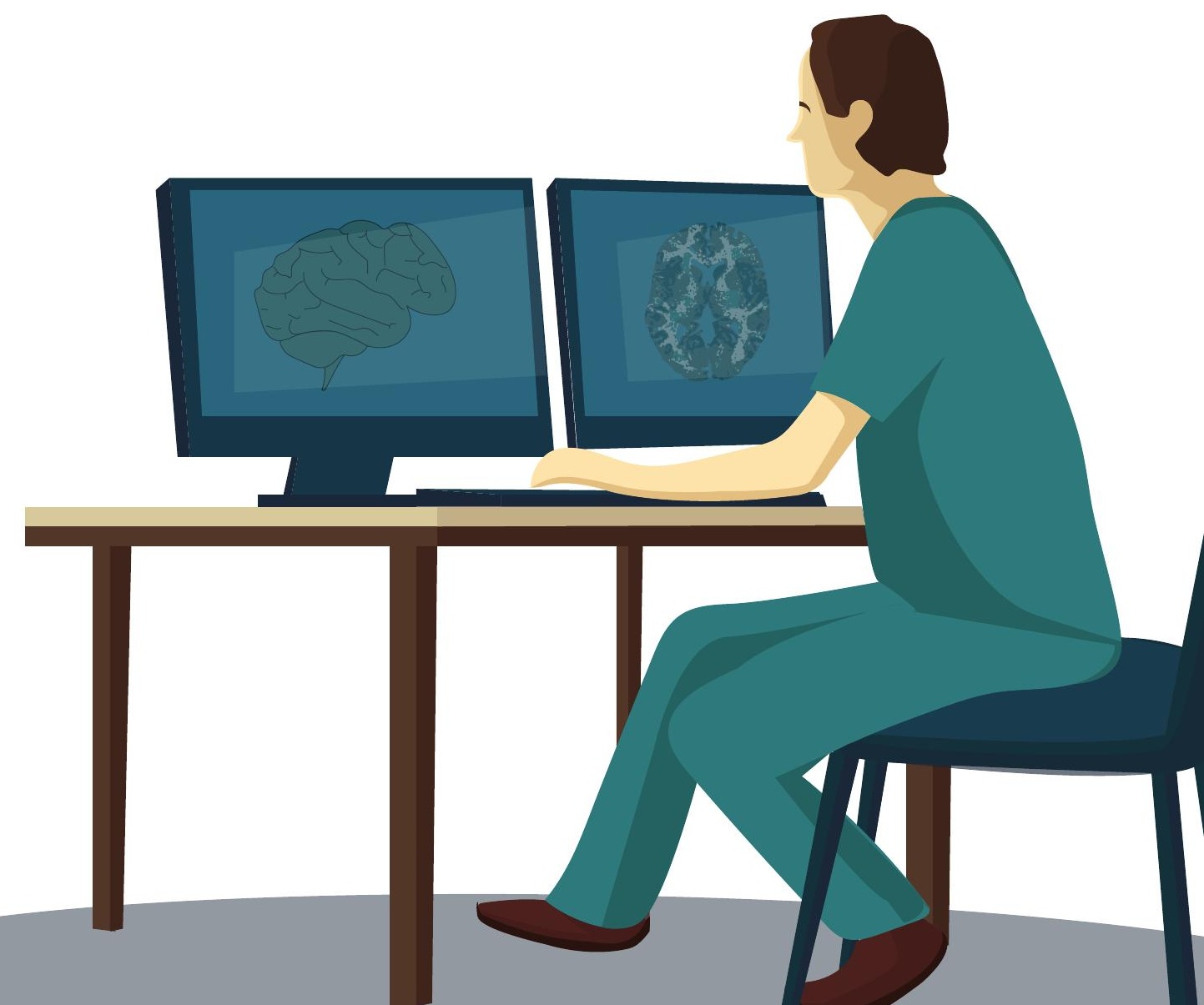


Part I | Technical aspects of dual-energy CT

\section{Abstract \\ Objectives}

To evaluate the image quality of virtual monochromatic images (VMI) reconstructed from dual-energy dual-source non-contrast head CT with different reconstruction kernels.

\section{Methods}

Twenty-five consecutive adult patients underwent non-contrast dual-energy CT. VMI were retrospectively reconstructed at 5-keV increments from 40-140 keV using quantitative and head kernels. CT number, noise levels (SD), signal-to-noise ratio (SNR) and contrast-to-noise ratio (CNR) in the gray and white matter and artifacts using the posterior fossa artifact index (PFAl) were evaluated.

\section{Results}

CT number increased with decreasing VMI energy levels, and SD was lowest at 85 keV. SNR was maximized at $80 \mathrm{keV}$ and $85 \mathrm{keV}$ for the head and quantitative kernels, respectively. CNR was maximum at $40 \mathrm{keV}$; PFAI was lowest at 90 (head kernel) and 100 (quantitative kernel) keV. Optimal VMI image quality was significantly better than conventional CT.

\section{Conclusions}

Optimal image quality of VMI energies can improve brain parenchymal image quality compared to conventional CT but are reconstruction kernel dependent and depend on indication for performing non-contrast CT. 


\section{Introduction}

Dual-energy CT (DECT) is an established method to improve image contrast, reduce imaging artifacts, and perform multi-material decomposition[21,27,68]. DECT is based on the simultaneous acquisition of two CT datasets at different energies (i.e. at a low and high energy). Collection of high- and low-energy datasets allows energy-specific and therefore material-specific spectral attenuation curves to be obtained for any given CT voxel. The physical principle underlying DECT is the energy-dependence of materials; i.e. the attenuation of a material changes with the incidence energy. The DECT dataset can then be used to generate different images, such as virtual non-contrast CT images[25,69,70], iodine maps[71,72] or virtual monochromatic images (VMI)[30,73].

Virtual monochromatic images[74-77] have the potential to increase the conspicuity of cerebral pathology on non-contrast head CT[23,24,43,50,78], by its ability to reduce streak artifacts and beam-hardening artifacts, or to improve contrast attenuation in comparison to conventional CT imaging. However, VMI techniques and reconstruction algorithms vary between manufacturers. This means that optimal monochromatic energy levels cannot be translated from one manufacturer to the other[79]. This means that for every manufacturer an extensive evaluation of image quality in VMI images is required. For the dual-source CT scanner, the image quality of monochromatic images has been well characterized in pediatric patients[80], but not in adults. In addition, the use of different reconstruction kernels on image quality in VMI has not been addressed in literature. We hypothesize that adult brain DECT will require unique virtual monochromatic reconstruction energies to optimize image quality as compared to previously determined in children. In addition, image quality will also have a reconstruction kernel dependency. To determine the quality of monochromatic reconstructions in adult patients, we present a quantitative analysis of the image quality of virtual monochromatic images reconstructed from non-contrast DECT images using a common quantitative kernel and a dedicated head kernel.

\section{Materials and Methods}

\section{Patients}

We retrospectively identified 25 consecutive patients who underwent DECT on our institutional dual-source CT (Somatom Force, Siemens Healthineers, Erlangen, Germany). Inclusion criteria were at least 18 years old, presenting with clinical suspicion of stroke, and MRI was performed within 48 hours to evaluate presence of ischemia. These are patients in which we routinely acquire dual-energy CT data as part of our standard-of-care CT protocol. This study was approved the Stanford University institutional review board and complied with the Health Insurance Portability and Accountability Act. The need for informed consent was waived. 


\section{Imaging protocol}

Non-contrast dual-energy head CTS (NCCT) were acquired helically using tube voltages of 80 and $140 \mathrm{kVp}$ with a tin filter, and tube currents of 640 and $320 \mathrm{mAs}$, respectively. Dual-energy $C T$ protocols were prepared to maintain dose neutrality with respect to single-energy protocols at our institution. Based on a $16 \mathrm{~cm}$ International Electrochemical Commission (IEC) head dosimetry phantom, the volume CT dose index $\left(C T D I_{v o l}\right)$ for each scan was $59.8 \mathrm{mGy}$. The acquisition and reconstruction parameters are shown in Table 4.1. From the high- and low-energy reconstructions, VMI reconstructions were generated in 5-keV increments from 40 to $140 \mathrm{keV}$ resulting in a total number of $21 \mathrm{VMI}$ reconstructions. Each reconstruction was generated using both a quantitative (Q34s) and a head (J30s) reconstruction kernel (Syngo.via, Siemens Healthineers, Erlangen, Germany). The Q34s kernel is designed specifically for DECT imaging with beam-hardening correction for bone. Both kernels are so-called standard "medium smoothing" kernels, translating to a standard resolution and a moderate smoothing effect on the image, with subsequent improvements in contrast detail and reduced noise levels, but poorer edge definition relative to a harder kernel.

Table 4.1 | Dual-energy non-contrast CT acquisition and reconstruction parameters.

\begin{tabular}{lllllll}
\hline $\begin{array}{l}\text { Scan } \\
\text { type }\end{array}$ & Pitch & $\begin{array}{l}\text { Beam collimation } \\
(\mathrm{mm})\end{array}$ & $\begin{array}{l}\text { Rotation } \\
\text { time }(\mathrm{s})\end{array}$ & $\begin{array}{l}\text { Slice } \\
\text { thickness }(\mathrm{mm})\end{array}$ & Kernel & Matrix \\
\hline Helical & 1.0 & $40 \times 0.6(24.0)$ & 1.0 & 3.0 & Q34s/J30s & $512 \times 512$ \\
\hline
\end{tabular}

\section{Quantitative analysis}

For every patient, one conventional NCCT series, which is a linear combination of both images from the two sources and $21 \mathrm{VMI}$ reconstructions were obtained per kernel resulting in a total of 44 series of image volumes per patient. Similar to prior studies[23,80], a total of 12 regions of interest (ROIs) of $25 \mathrm{~mm}^{2}$ were drawn manually, and then adjusted to avoid partial volume averaging with adjacent tissue. If the patient had a visible infarct, the ROls were drawn outside the infarcted area.

Paired ROIs were manually placed by an experienced radiology trainee (FK; 3 years of experience) bilaterally in the cortical gray matter of the frontal and parietotemporal lobes, the juxtacortical white matter, the deep thalamic gray matter, and the white matter of the posterior limb of the internal capsule. In addition, a $215 \mathrm{~mm}^{2} \mathrm{ROI}$ was placed in the interpretrous region of the posterior fossa on the slice with the greatest beam-hardening or streak artifact. For each ROI, the mean CT number and noise level was recorded in Hounsfield units (HU). The noise level (SD) was defined as the standard deviation of the CT numbers for each ROI. In addition, a measure for signal-to-noise ratio (SNR) was calculated for gray and white matter in every ROI:

$$
S N R_{\text {matter }}=\frac{\overline{H U}_{\text {matter }}}{S D_{\text {matter }}}
$$


To measure gray-white matter differentiation between adjacent gray and white matter ROI measurements, a gray-white matter contrast-to-noise ratio (CNR) was calculated as:

$$
C N R=\frac{\left(\overline{H U_{G M}}-\overline{H U_{W M}}\right)}{\sqrt{\left(S D_{G M}^{2}+S D_{W M}^{2}\right)}} .
$$

The posterior fossa artifact index (PFAI) was additionally calculated using a previously described method[81]. The PFAl is defined as the standard deviation of the ROI in the posterior fossa, as a measure of $\mathrm{HU}$ variation, which thereby estimates the severity of beam-hardening or streak artifact.

\section{Statistical analysis}

Patient characteristics were reported as median with interquartile range (IQR) for continuous variables and frequencies with percentages for dichotomous variables. Measures of CT number, noise, SNR, CNR and PFAI were reported as mean \pm standard deviation. We compared measures of CT number, noise, SNR, CNR and PFAI between VMI reconstructions by using repeated-measures of analysis of variance and a posthoc Dunnett test. The maximum SNR, CNR and minimum PFAI were compared to the conventional NCCT measurements using paired t-tests. Differences between each reconstruction kernel for CT number, noise, SNR, CNR and PFAI were analyzed with paired t-tests and the Bonferroni correction was applied to adjust for multiple testing. All analyses were conducted in SPSS version 25.0 (IBM, Armonk, NY, USA).

\section{Results}

\section{Baseline patient characteristics}

Twenty-five patients (median age of 63 (IQR 44 - 82) years, 36\% female) with suspicion of stroke underwent non-contrast DECT. Fifteen patients were diagnosed with having a stroke on MRI ( $\leq 24$ hours of onset), with a median presentation National Institutes of Health Stroke Scale (NIHSS) of 2 (IQR 0 - 8). There were $2(8 \%)$ patients with known coronary artery disease, 5 (20\%) with atrial fibrillation, 4 (16\%) with diabetes, 14 (56\%) with hypertension, 10 (40\%) with hyperlipidemia, 2 (8\%) with a prior cerebrovascular accident or intracranial hemorrhage, 5 (20\%) were smokers and 13 (52\%) were on antiplatelet or anticoagulant therapy.

\section{Quantitative analysis}

In Figure 4.1, an example of a patient VMI reconstruction is shown, illustrating the differences in image quality, which will be evaluated. 


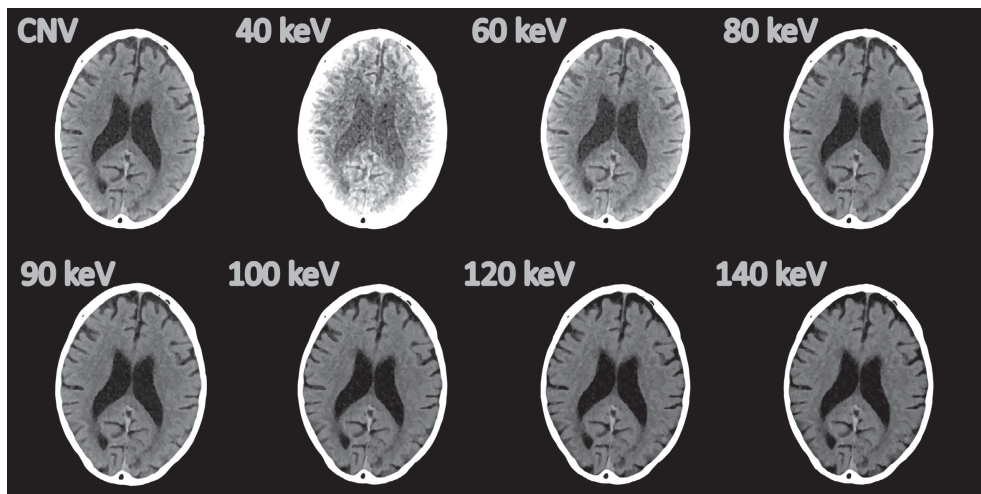

Figure 4.1 | J30s kernel reconstructed conventional (CNV) and VMI NCCT at 40, 60, 80, 90, 100, 120 and $140 \mathrm{keV}$. Noise is more prone in the $40 \mathrm{keV}$, compared to the $140 \mathrm{keV}$ reconstructions. The window level/width is $40 / 80 \mathrm{HU}$ for all reconstructions. The example shown here is exemplary for the Q34s kernel.

\section{CT number}

The mean CT number of the gray and white matter of each VMI reconstruction is plotted in Figure 4.2. There was a similar gradual decrease in the gray and white matter CT number with increasing reconstruction energy. Mean gray matter CT number at 40 keV was $52.20 \pm 12.25 \mathrm{HU}$ and $72.94 \pm 29.68 \mathrm{HU}$ for the Q34s and J30s kernels, which decreased to $29.15 \pm 2.95 \mathrm{HU}$ and $27.36 \pm 2.64 \mathrm{HU}$ at $140 \mathrm{keV}$, respectively. There was a significant difference in CT number between the J30s kernel compared to the Q34s kernel at all energy levels ( $p<0.001)$ except $100 \mathrm{keV}(p>0.875)$. Mean white matter CT number at $40 \mathrm{keV}$ was $42.70 \pm 7.86 \mathrm{HU}$ and $46.53 \pm 8.74 \mathrm{HU}$ for the Q34s and J30s kernels, respectively, which decreased to $27.64 \pm 2.89 \mathrm{HU}$ and $27.30 \pm 3.70 \mathrm{HU}$ at 140 keV, respectively. There was a small but significant difference in white matter CT number between the J30s and Q34s kernel at 40 - 90, 135 and $140 \mathrm{keV}(\mathrm{p}<0.002)$.

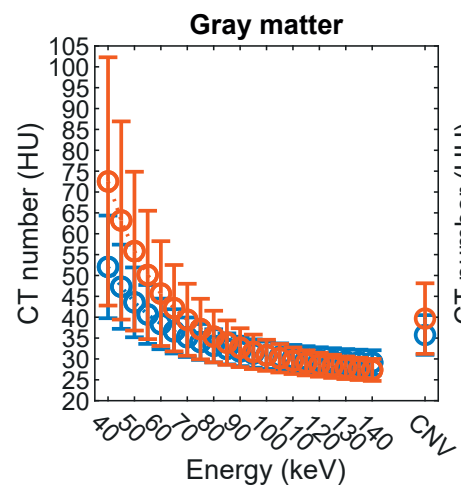

(1) Q34s $\quad$ (1) J30s

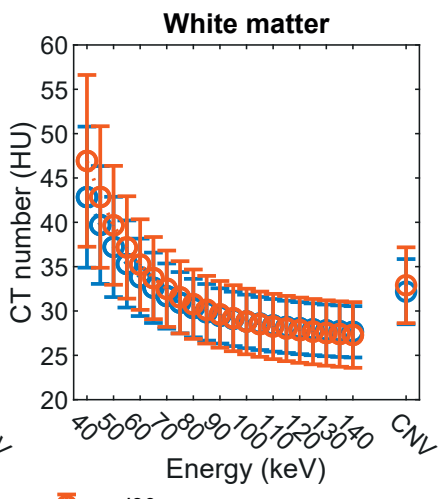

Figure 4.2 | Mean \pm standard deviation $\mathrm{CT}$ number (HU) of the gray and white matter for the different virtual monochromatic images. The conventional NCCT (CNV) CT number is shown for reference purposes. 
CT noise

From 40 to $85 \mathrm{keV}$, quantitative measures of noise in the gray and white matter decreased with increasing energy (Figure 4.3). Mean gray matter noise at $40 \mathrm{keV}$ were $8.44 \pm 2.03 \mathrm{HU}$ and $9.25 \pm 2.77 \mathrm{HU}$ for Q34s and J30s kernels, respectively. Minimal noise was observed at $90 \mathrm{keV}(3.41 \pm 0.80 \mathrm{HU})$ using the Q34s kernel. The minimum noise at $90 \mathrm{keV}$ was not significantly lower compared to $85 \mathrm{keV}$ and $95-140 \mathrm{keV}$ ( $p>0.650)$, whereas the other energies were significantly higher $(p<0.001)$. Minimal noise was observed at $85 \mathrm{keV}(3.14 \pm 0.67 \mathrm{HU})$ using the J30s kernel. Noise at $85 \mathrm{keV}$ was significantly lower from all other monochromatic reconstructions $(p<0.001)$, excepting the 80, 90 and $95 \mathrm{keV}$ reconstructions ( $p>0.040)$. There was a significant difference between the J30s and Q34s kernel for 40 and 65 - $100 \mathrm{keV}(p<0.001)$. Mean white matter noise measurements demonstrated a similar pattern, but with overall lower noise levels. Minimal noise was observed at $85 \mathrm{keV}$ for both the Q34s and J30s kernels (3.08 0.62 $\mathrm{HU}$ and $2.93 \pm 0.61 \mathrm{HU}$, respectively). The noise at $85 \mathrm{keV}$ was significantly lower than the noise at all other VMI energies ( $p<0.001)$, except at 90 - $140 \mathrm{keV}(p=1.000)$ for the Q34s kernel. For the J30s kernel, the $85 \mathrm{keV}$ reconstruction is significantly lower from all monochromatic reconstructions ( $p<0.001$ ), except 80, $90-100 \mathrm{keV}(p>0.002)$. The noise for the J30s kernel is significant lower for 40 - $110 \mathrm{keV}(p<0.001)$ compared to the Q34s kernel.
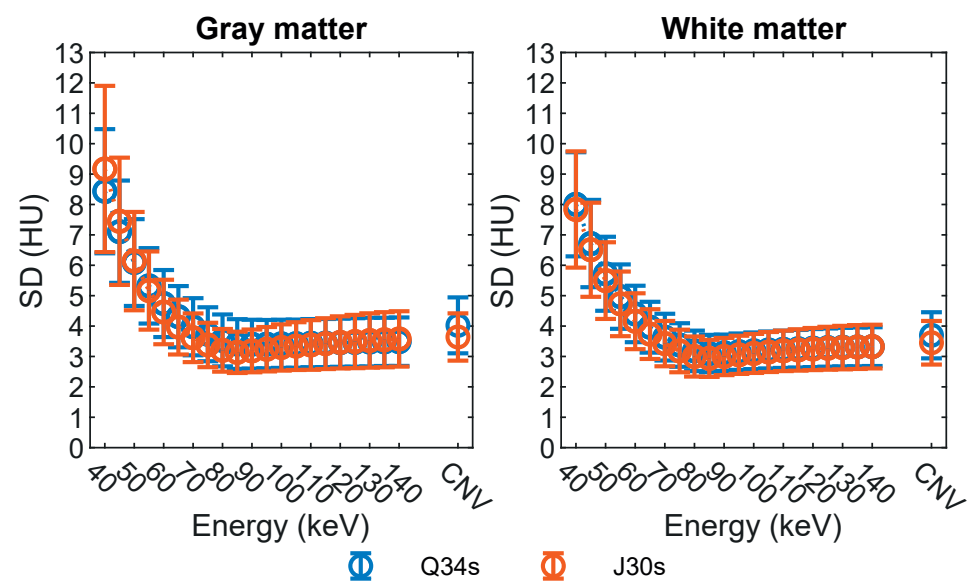

Figure 4.3 Mean \pm standard deviation noise levels (SD) (HU) of the gray and white matter for virtual monochromatic images of 40 to $140 \mathrm{keV}$. The conventional NCCT (CNV) noise is shown for reference purposes. 
Signal-to-noise ratio (SNR)

Gray matter SNR ranged from $6.56 \pm 2.34$ to $9.95 \pm 2.41$ and $8.16 \pm 2.34$ to 11.58 \pm 3.35 with the Q34s and J30s kernels, respectively. An example of VMI using both kernels is shown in Figure 4.4. The maximum gray matter SNR was observed at 85 keV using the Q34s kernel (9.95 \pm 2.41 ; Figure 4.5), which is significantly higher from all other monochromatic reconstructions $(p<0.001)$, except for the 80 and $90 \mathrm{keV}$ reconstructions ( $p>0.030)$. For the J30s kernel, the maximum is at $80 \mathrm{keV}(11.58 \pm 3.35)$, which is significantly higher compared to 40 - 55 and 90 - $140 \mathrm{keV}(p<0.001)$. There is a significant increase in SNR between Q34s and J30s reconstruction kernels for the low energy levels $(40-100 \mathrm{keV})(p<0.001)$. White matter SNR ranged from $5.56 \pm 1.48$ to $10.00 \pm 2.09$ and $6.28 \pm 1.75$ to $10.69 \pm 2.43$ with a maximum at $85(10.00 \pm 2.09)$ and $80 \mathrm{keV}(10.69 \pm 2.43)$ for the Q34s and J30s kernel, respectively. The maximum for the Q34s kernel is significantly higher for all VMI energy levels $(p<0.001)$, except for 80 $\mathrm{keV}(\mathrm{p}=1.000)$. For J30s, all but 85 and $90 \mathrm{keV}(p>0.095)$ are significantly lower than the maximum of $80 \mathrm{keV}(p<0.001)$. As compared to the Q34s kernel, there is a small but significant increase in SNR for the 40 - $105 \mathrm{keV}$ monochromatic reconstructions using the J30s kernel $(p<0.002)$. The maximum SNR of the gray and white matter is significantly higher than that of the conventional NCCT $(p<0.001)$, except for the gray matter SNR of the J30s kernel ( $p=0.168)$.

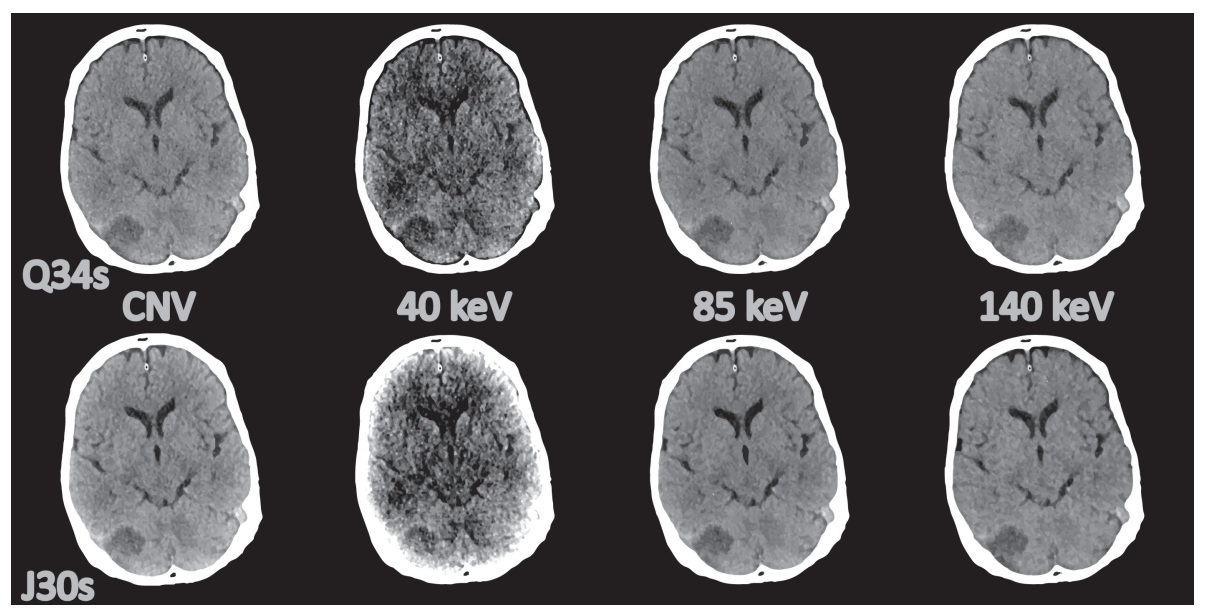

Figure 4.4 SNR is higher at $85 \mathrm{keV}$ for both Q34s and J30s kernel, compared to $40 \mathrm{keV}$ or 140 $\mathrm{keV}$. The J30s increases the SNR compared to the Q34s kernel, even though the noise increases too. The window level/width is 40/80 HU for conventional NCCT (CNV) and $85 \mathrm{keV}, 35 / 80 \mathrm{HU}$ for $140 \mathrm{keV}$ and 65/80 HU (Q34s) and 70/80 HU (J30s) for $40 \mathrm{keV}$ to compensate for differences in mean CT number. An infarction is visible in the right infratentorial brain. 

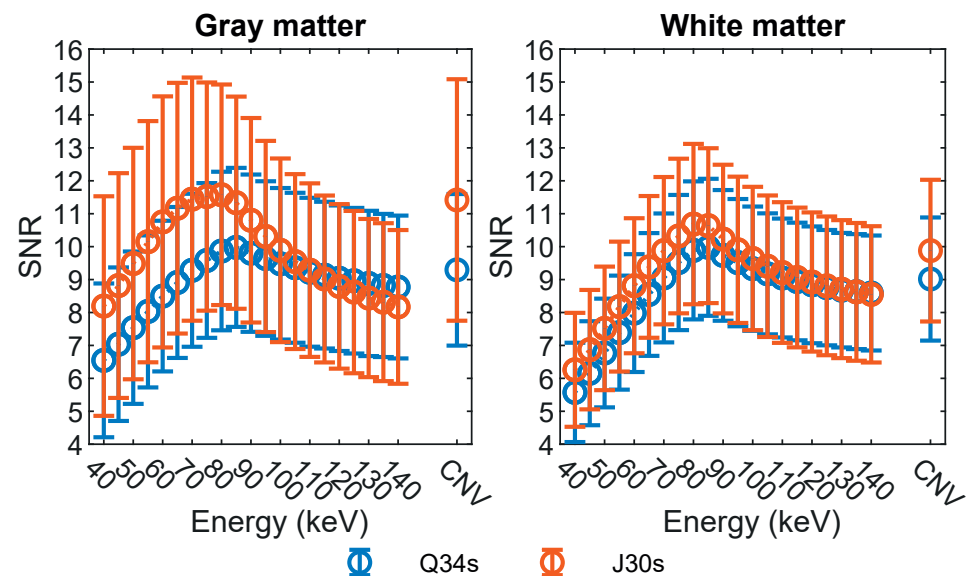

Figure 4.5 | Mean \pm standard deviation SNR of the gray and white matter for $40-140 \mathrm{keV}$ monochromatic CT images. In addition, the SNR for the conventional NCCT (CNV) is shown as well.

\section{Contrast-to-noise ratio (CNR)}

The mean CNR ranged from $0.81 \pm 0.63$ (140 keV) to $1.67 \pm 0.72$ (40 keV) and $0.71 \pm 0.78$ (140 keV) to $3.10 \pm 1.45$ (40 keV) for Q34s and J30s, respectively (Figure 4.6). The CNR was greatest at $40 \mathrm{keV}(1.67 \pm 0.72$ (Q34s) and $3.10 \pm 1.45$ (J30s)), which was significantly greater than the CNR at $100 \mathrm{keV}$ and higher energy levels $(p<0.002)$ for Q34s. The maximum was significantly greater than the CNR of $65-140$ keV for J30s $(p<0.001)$. There was a significant increase in the CNR for the J30s kernel as compared to the Q34s kernel for reconstructions ranging from 40 - $110 \mathrm{keV}(p<0.001)$. The maximum CNR at $40 \mathrm{keV}$ was significantly greater than the CNR of the conventional NCCT $(1.44 \pm 0.62$ (Q34s) and $2.49 \pm 1.04(J 30 s)$ ) for both kernels $(p<0.001)$.

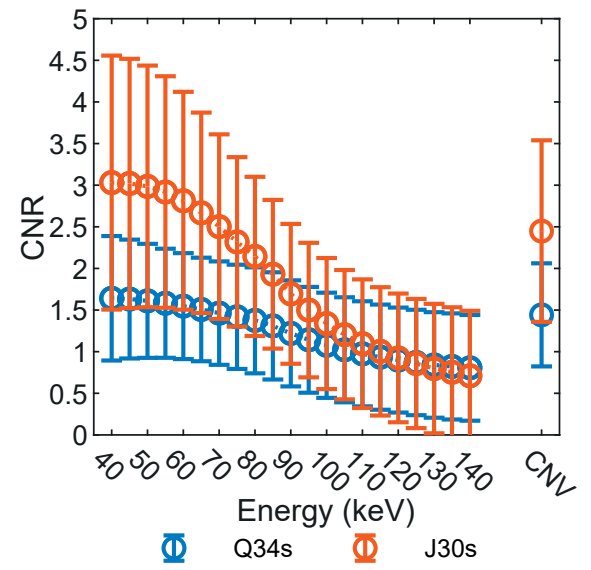

Figure 4.6 | Mean \pm standard deviation CNR of the different reconstruction kernels for monochromatic CT images. The CNR of the conventional NCCT (CNV) are shown as well, which is significantly lower than the maximum CNR of the VMI. 
Posterior fossa artifact index (PFAl)

The PFAl ranged from $4.98 \pm 1.63 \mathrm{HU}$ to $13.20 \pm 2.75 \mathrm{HU}$ and $5.14 \pm 1.79 \mathrm{HU}$ to 16.39 $\pm 3.79 \mathrm{HU}$ for the Q34s and J30s kernels, respectively (Figure 4.7). An example of the PFAl differences is shown in Figure 4.8. The minimum PFAl, or the smallest amount of artifacts, was observed at $90 \mathrm{keV}(5.14 \pm 1.79 \mathrm{HU})$ using the J30s kernel and $100 \mathrm{keV}$ $(4.98 \pm 1.63 \mathrm{HU})$ using the Q34s, which was significantly lower than the PFAl at $<80 \mathrm{keV}$ $(p<0.001)$ for both kernels. The PFAl is lower for Q34s at all energies compared to J30s, the difference is significant for all energies ( $p<0.002)$, except 55 - $105 \mathrm{keV}(p>0.004)$. The minimum PFAI is significantly lower than the PFAI of the conventional NCCT (5.98 $\pm 1.69 \mathrm{HU}(\mathrm{Q} 34 \mathrm{~s})$ and $6.15 \pm 1.57 \mathrm{HU}(\mathrm{J30s})$ ) for both kernels $(p<0.001)$.

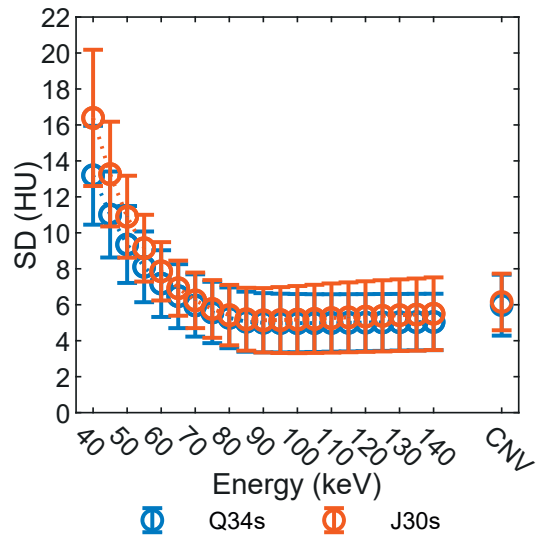

Figure 4.7 | Mean \pm standard deviation of the standard deviation of the CT number (HU) of the posterior fossa for the different reconstruction kernels for the virtual monochromatic images and conventional NCCT reconstruction (CNV).

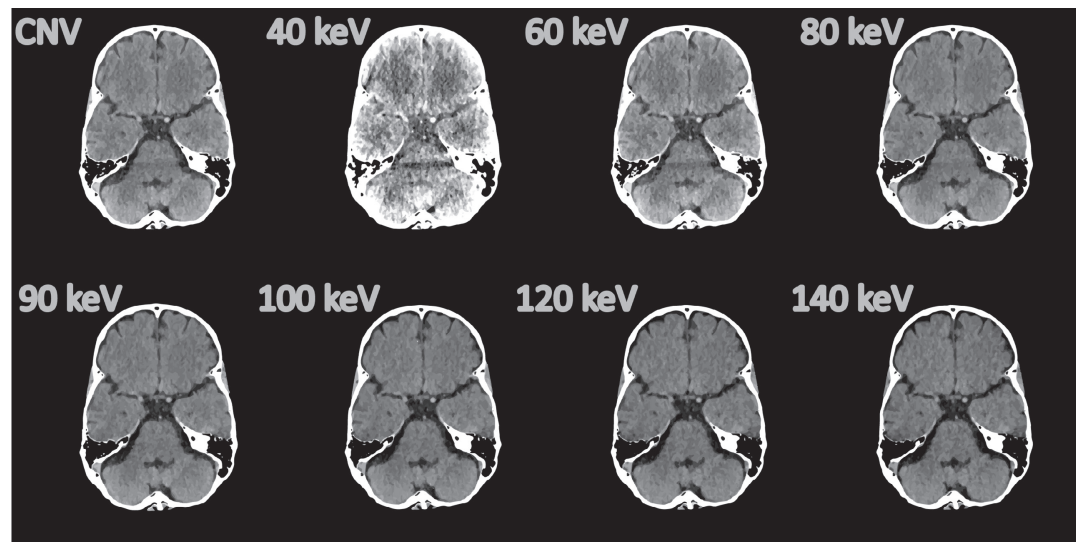

Figure 4.8 Example of the change in noise in the posterior fossa for conventional NCCT (CNV), and for 40, 60, 80, 90, 100, 120 and $140 \mathrm{keV} \mathrm{VMI}$. The J30s reconstruction kernel is shown here. The window level/width is 40/80 HU for all images. The images shown here are exemplary for the Q34s kernel. 


\section{Discussion}

We found that depending on the used reconstruction kernel, DECT noise levels are reduced at 85 or $90 \mathrm{keV}$, and SNR was maximized at 80 and $85 \mathrm{keV}$. CNR was highest at $40 \mathrm{keV}$, but so was the PFAl. The PFAl was the smallest at 90 or $100 \mathrm{keV}$ depending on the kernel.

To the best of our knowledge, this is the first study looking at virtual monochromatic images in adults using dual-source non-contrast DECT. In a previous study that evaluated the image quality of VMI in children[80], a similar trend for the CT numbers of the gray and white matter was observed as compared to this study. Noise levels, however, were substantially lower compared to the previous study conducted in pediatric patients[80]. The reduction in noise level was associated with a substantial increase in SNR in both the gray and white matter. The difference in noise levels can be explained by the acquisition settings; the peak voltage was similar, but the mAs were substantially higher in our study. This resulted in substantially higher dose levels in our study. This is likely because radiologists tend to accept images of lower quality in pediatric patients because of the focus on dose reduction, whereas, in adult patients with suspected stroke, image quality is paramount to detect subtle signs of early ischemia.

Interestingly, the CNR values we observed were comparable to the values observed in the pediatrics paper. In pediatric patients myelination may be incomplete depending on the age of the patients, and may be of variable attenuation due to different waterfat content ratios adding to the heterogeneity in their study, whereas in adults without underlying WM pathology, we have a relatively uniform WM attenuation. This may allow separate $\mathrm{VMI}$ reconstructions to be optimized for patient age, which is for a future study. The maximal CNR was observed at $40 \mathrm{keV}$ in adults as opposed to $60 \mathrm{keV}$ in pediatric patients. This shift is due to an increase in white matter attenuation at low keV. The maximum CNR is also not significantly larger than the CNR found up until 65 or $100 \mathrm{keV}$ depending on the reconstruction kernel, suggesting comparable contrast in the images between the different VMI up until 65 or $100 \mathrm{keV}$.

The PFAl is highest in the low keV (40 - $60 \mathrm{keV}$ ) VMI, and is minimal at 90 (J30s) or 100 (Q34s) keV. Prior research[82] showed that higher VMI energies can be used to reduce beam hardening artifacts. This can be explained by the fact that generation of high keV monochromatic images involves a higher contribution of the high kVp image, cancelling out beam hardening artifacts. The maximum SNR at 80 and $85 \mathrm{keV}$, and the minimum PFAl at 90 or $100 \mathrm{keV}$ suggests that the indication for the head NCCT determines the required VMI energy. If a high SNR is required, a slightly lower keV is chosen, whereas if a reduction in artifacts is required a higher keV is needed. 
The use of a head kernel resulted in a significant increase in SNR and CNR for the lower energies (40 - $100 \mathrm{keV}$ ) compared to the quantitative kernel. The GM-WM differentiation increased significantly using the head kernel. The J30s kernel increased GM CT number significantly, while maintaining a similar WM CT number and noise levels as compared to the Q34s kernel. The maximum, however, shifted slightly to a different VMI energy. For the SNR, maximum shifted from $80 \mathrm{keV}$ (Q34s) to $85 \mathrm{keV}$ (J30s) and for PFAI from 100 (Q34s) to $90 \mathrm{keV}$ (J30s). Because the maximum SNR, CNR and minimum PFAl are VMI energy dependent, but also reconstruction kernel dependent, the selection of a reconstruction kernel should be carefully considered and investigated. If the scan is used for small malformations close to the skull or in the infratentorial area a quantitative kernel at $100 \mathrm{keV}$ would be preferred because the artifact index is minimized at this energy level. With more supratentorial diagnostics, e.g. hemorrhage detection or identification of brain masses in which high SNR and CNR are more important a reconstruction with a head kernel at $65 \mathrm{keV}$ would be preferred, which is a trade-off between maximum CNR and maximum SNR. In addition, the exaggeration of the graywhite matter distinction using the head kernel could be advantageous when assessing ischemic changes in the brain.

Our study has several limitations, including its retrospective design and relatively small number of patients. Also, we solely focused on assessing image quality parameters. Conclusions on diagnostic accuracy in a clinical setting cannot be drawn from the presented data. Lastly, in this study we chose to use CT number to estimate the SNR, to be able to compare with prior research. Of course if a different metric or definition for signal is chosen (e.g. the linear attenuation coefficient), this may alter the SNR value. This would, however, not chance the general conclusions of this study.

In conclusion, the use of virtual monochromatic images can improve brain parenchymal image quality compared to conventional NCCT. The desired energy level for the VMI reconstruction should be based on the indication for performing the NCCT. To minimize beam-hardening and streak artifacts an optimal energy of 90 or $100 \mathrm{keV}$ should be chosen, whereas if one seeks to maximize SNR and CNR one should look at 65 or 80 keV depending on the used reconstruction kernel. This shows that the use of different reconstruction kernels can affect image quality significantly, and therefore must be selected carefully. 
\begin{tabular}{l|l|l} 
Image quality of non-contrast virtual monochromatic images & Chapter $\mathbf{4}$
\end{tabular} 



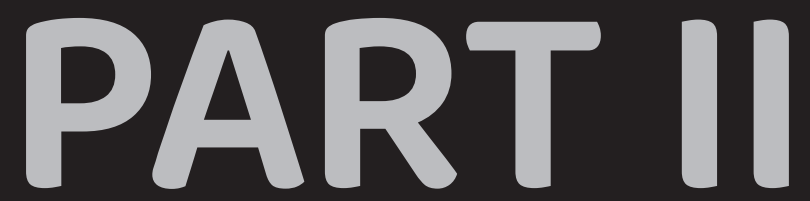

\section{DUAL-ENERGY CT IN STROKE IMAGING}





\section{CHAPTER 5}

\section{VIRTUAL MONOCHROMATIC NON-CONTRAST BRAIN CT FOR DETECTION OF STROKE}

Based on

F van Ommen, JW Dankbaar, G Zhu, DN Wolman, JJ Heit, F Kauw, E Bennink, HWAM de Jong and M Wintermark (2020). Virtual monochromatic dual-energy CT reconstructions improve detection of cerebral infarct in patients with suspicion of stroke. Neuroradiology, in press.

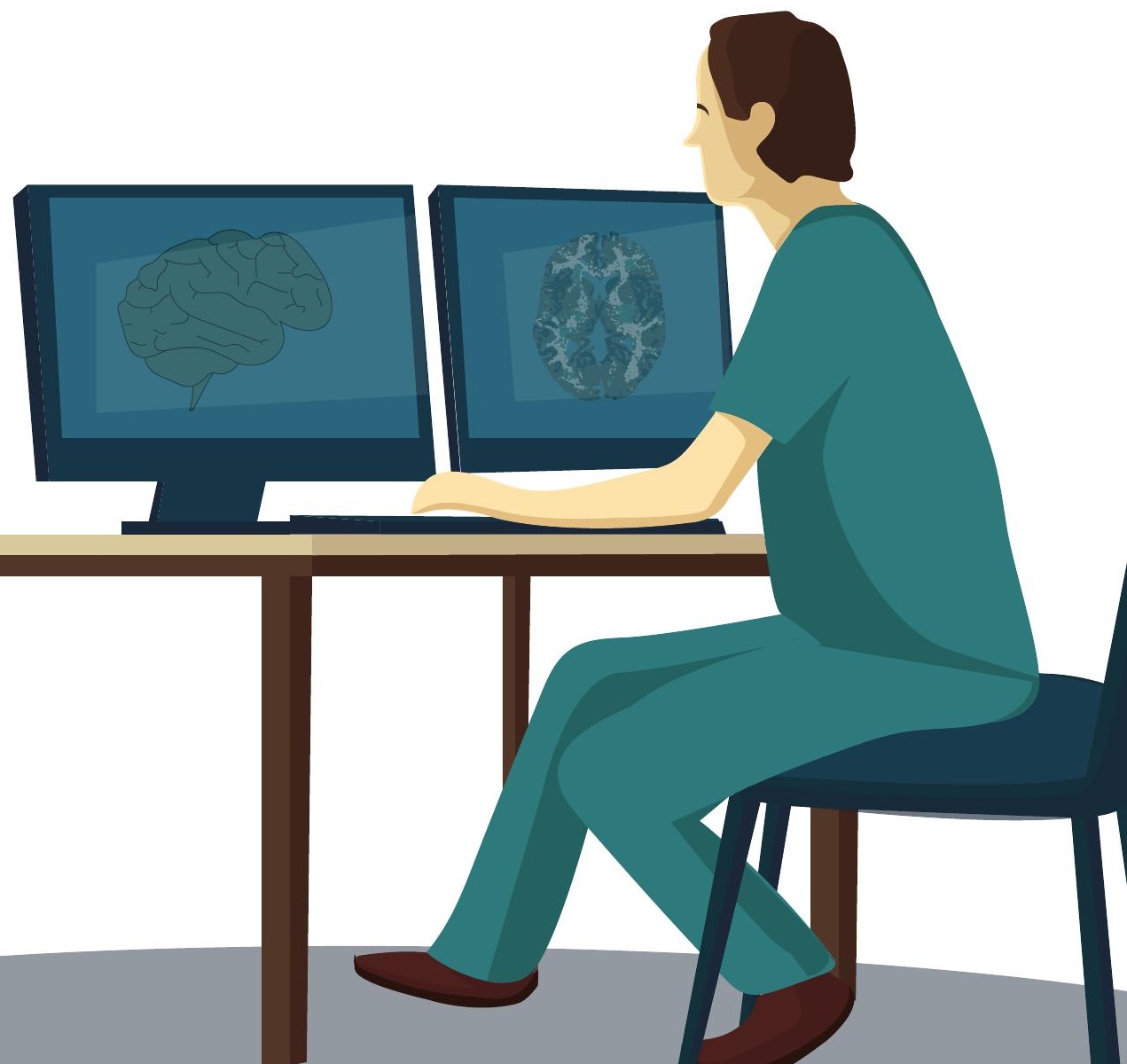


Part II | Dual-energy CT in stroke imaging

\section{Abstract \\ Objectives}

Early infarcts are hard to diagnose on non-contrast head CT. Dual-energy CT (DECT) may potentially increase infarct differentiation. The optimal DECT settings for differentiation were identified and evaluated.

\section{Methods}

One-hundred-and-twenty-five consecutive patients who presented with suspected acute ischemic stroke (AIS) and underwent non-contrast DECT and subsequent diffusion-weighted imaging (DWI) were retrospectively identified. The DWI was used as reference standard. First, virtual monochromatic images (VMI) were reconstructed from 40 - $140 \mathrm{keV}$ and scored by two readers for acute infarct. Sensitivity, specificity, positive and negative predictive values for infarct detection were compared and a subset of VMI energies were selected. Next, for a separate larger cohort of suspected AIS patients, conventional non-contrast CT (NCT) and selected VMI were scored by two readers for the presence and location of infarct. The same statistics for infarct detection were calculated. Infarct location match was compared per vascular territory. Subgroup analyses were dichotomized by time from last-seen-well to CT imaging.

\section{Results}

80 - 90 keV VMI were marginally more sensitive (36.3 - 37.3\%) than NCT (32.4\%; $p>0.680$ ), with marginally higher specificity (92.2 - 94.4 vs 91.1\%; $p>0.509)$ for infarct detection. Location match was superior for VMI compared to NCT (28.7 - 27.4 vs 19.5\%; $\mathrm{p}$ 0.010). Within 4.5 hours from last-seen-well, $80 \mathrm{keV}$ VMI more accurately detected infarct (58.0 vs 54.0\%) and localized infarcts (27.1 vs 11.9\%; $p=0.004$ ) than NCT, whereas after 4.5 hours 90 keV VMI was more accurate (69.3 vs 66.3\%).

\section{Conclusions}

Non-contrast 80 - 90 keV VMI best differentiates normal from infarcted brain parenchyma. 


\section{Introduction}

Non-contrast CT is the mainstay in the initial evaluation of patients with suspicion of acute ischemic stroke (AIS)[83]. However, the sensitivity of non-contrast CT is limited for the detection of acute brain infarct[84]. The reference standard for infarct detection is MRI with diffusion-weighted imaging (DWI) $[85,86]$. DWI allows for detection of cytotoxic edema within infarcted tissue with high sensitivity, while CT is limited to detecting subtle changes in water content between infarcted and normal brain parenchyma.

Dual-energy CT (DECT) advances CT imaging by improving upon conventional noncontrast head CT (NCT) by acquiring data with two separate energy spectra, which allows improved contrast resolution, reduced image noise and beam-hardening artifacts, and spectral separation of constituent materials at equivalent dose[21,87]. Virtual monochromatic CT images (VMIs) can be derived from source DECT data[30,73,88], and reflect the tissue properties of a scan acquired at a single specific monochromatic energy level. Leveraging different monochromatic reconstructions may help accentuate differences in energy dependent attenuation differences between similar materials e.g. normal brain tissue and ischemic/edematous brain tissue. DECT VMI have demonstrated improved contrast-to-noise profiles and reduced beam hardening artifact relative to NCT[23,24,43,50,78,80]. The potential of DECT for the visualization of brain edema has been investigated in earlier studies[89-91]. In these studies elaborate reconstruction methods were required, whereas VMI is a standard derivative of DECT imaging and broadly applicable to any vendor's software. The use of VMI in the detection of cerebral infarct has not been established. We sought to identify the non-contrast VMI energy which best differentiates normal from infarcted brain parenchyma, and to determine if this VMI can more sensitively and specifically identify infarct in patients with suspected AIS compared to NCT.

\section{Materials and Methods}

\section{Patients}

This study was approved by the Stanford University institutional review board which waived the need for informed consent, and data collection complied with the Health Insurance Portability and Accountability Act. We retrospectively enrolled consecutive patients between October 13, 2018 and April 18, 2019 with suspected AIS who underwent non-contrast DECT and subsequent MRI with DWI within 48 hours. Inclusion criteria were: patient age $\geq 18$ years and presentation within 24 hours of symptom onset. Exclusion criteria were: technical failure of DECT, significant metal artifact limiting interpretation or corrupted DWI. Baseline clinical data was collected, including age, sex, presentation National Institutes of Health Stroke Scale (NIHSS), time since last-seenwell, time to initial DECT imaging, and time to subsequent MRI. 


\section{Imaging protocol}

All patients were imaged using a dual-source Somatom Flash CT scanner (Siemens Healthineers, Erlangen, Germany). Non-contrast dual-energy CT protocols are dose neutral with respect to single-energy acquisitions at our institution. The volume CT dose index $\left(C T I_{\text {vol }}\right)$ for each scan was $59.8 \mathrm{mGy}$, and was based on a $16 \mathrm{~cm}$ International Electrochemical Commission (IEC) head dosimetry phantom. The scan parameters were as follows: Tube A: $80 \mathrm{kVp}$ and $640 \mathrm{mAs}$; Tube B: $140 \mathrm{kVp}$ with a tin filter and $320 \mathrm{mAs}$, beam collimation of $40 \times 0.6 \mathrm{~mm}$, a 1.0 s rotation time, matrix size $512 \times 512$, and a pitch of 1.0. Images were reconstructed at $3 \mathrm{~mm}$ using a medium smoothing Q34s kernel. Non-contrast DECT VMI images were reconstructed using the Monoenergetic+ software module in Syngo.via (Siemens Healthineers, Erlangen, Germany).

\section{Study design}

Reference standard MRI (DWI) was obtained within 48 hours of each patient's index CT and was reviewed by a single neurointerventional radiologist with 7 years of experience (JJH). DWI imaging parameters included; TR 6000 ms, TE 78.2 ms, b-values: 0 and 1000, flip angle $90^{\circ}$, and $5 \mathrm{~mm}$ slice thickness. Each MRI was scored on a binary scale for presence of cerebral infarct, defined as focal parenchymal restricted diffusion. Cerebral infarcts were binned by location using a modified Alberta Stroke Program Early CT Score (mASPECTS)[92,93], in which additional regions corresponding to the posterior circulation (thalamus, superficial posterior cerebral artery, brainstem and cerebellum) and anterior cerebral artery territory (A1 anteriorly, and A2 posteriorly) are added. The A1 territory is anatomically bounded as the inferior ACA territory; while the A2 territory was bounded by the superior ACA territory.

Phase 1 of this study was used as an initial selection step to identify the VMI reconstruction energies best suited for infarct detection. Phase 2 compared these identified VMI reconstructions against NCT for the detection of cerebral infarct and qualitative infarct localization to identify VMI energies best for differentiation between normal and infarcted brain tissue.

\section{Phase 1 design}

In the first phase of the study, VMI were reconstructed at $10 \mathrm{keV}$ increments from 40 to $140 \mathrm{keV}$. VMI reconstructions were randomized and reviewed by a neuroradiologist and a neurologist (JWD and GZ, with 12 and 19 years of experience in radiology, respectively, and both more than 10 years of experience in stroke imaging) who were blinded to the reconstruction type, but did have $\mathrm{CT}$ indication information. Each reader reviewed a full axial image stack for each series with fixed window level and width settings. As CT numbers (HU) vary with tube voltage[94], the fixed window level (WL) for each reconstruction energy was visually identified by a neuroradiologist (MW) and set to maintain similar attenuation uniformity between series. 
The settings were as follows: 40 - $50 \mathrm{keV}$ (WL: $50 \mathrm{HU}), 60$ - $70 \mathrm{keV}$ (WL: $45 \mathrm{HU}), 80$ $110 \mathrm{keV}$ (WL: $40 \mathrm{HU}$ ), and $120-140 \mathrm{keV}$ (WL: $35 \mathrm{HU}$ ). Window width (WW) was set to $50 \mathrm{HU}$ for all reconstructions. Reviewers evaluated for the presence of acute cerebral infarct, and localized infarct foci using the mASPECTS system. Acute infarct was defined as a focal loss of gray-white matter differentiation, focal edema, or hypoattenuation without volume loss. Discrepant reviews were resolved by consensus. A number of VMI reconstructions, who were the most sensitive and specific for the detection of cerebral infarct relative to the reference standard MRI with DWI, were selected and used for comparison with NCT and used to identify the VMI energies that best differentiated normal from infarcted tissue.

Phase 2 design

In the second phase, two different independent reviewers scored a separate, larger cohort of patients presenting with suspicion for AIS. Each examination was reconstructed as a NCT (WL/WW: 40/50 HU) and the most sensitive and specific VMl energies identified in phase 1 (60 - $90 \mathrm{keV})$, and were presented in a randomized, blinded fashion using the same fixed window level and width settings as described in phase 1. An experienced neuroradiologist (JWD) and a neuroradiology fellow (DNW) scored each examination for evidence of acute infarct. Subgroup analyses of patients imaged in the early-window $(\leq 4.5$ hours after lastseen-well) and those presenting in the late-window ( $>4.5$ hours after last-seen-well) were performed to assess for time-dependent differences in infarct detection. We expect that infarcts in the early-window require different improvement in differentiation between similar attenuating tissue (fat, water and soft tissue), then infarcts in the late-window, and might have an influence on the required VMI energy for infarct detection. The volume of cerebral edema increases over time resulting in increased hypoattenuation on CT. At early time points the contrast between the edema and normal brain tissue is therefore less conspicuous than at later time points. Increasing the contrast between edema and normal brain tissue at different time points may therefore require different energies.

\section{Statistical analysis}

Baseline patient characteristics were compared between phase 1 and 2 using a MannWhitney $U$ test for continuous variables and a Chi-squared test for discrete variables. In phase 1, the sensitivity, specificity, positive predictive value (PPV), and negative predictive value (NPV) were calculated for the detection of cerebral infarct for each scored VMI reconstruction relative to the reference standard MRI with DWI. In phase 2, the sensitivity, specificity, PPV, NPV, and accuracy for the detection of cerebral infarct was calculated for the selected VMI reconstruction and the NCT relative to the reference DWI. Sensitivity and specificity was compared between NCT and VMI using the Chi-squared test. Regional infarct localization between DWI and CT was calculated as the infarct location match (ILM), which is defined as the percentage of true positive detections of infarct on CT compared to the total of positive detections for infarct on DWI in an anatomical region. 
ILM was compared between NCT and VMI for the anterior cerebral artery (ACA; A1 and A2), basal ganglia and insular cortex (BG; caudate, lentiform nucleus, internal capsule and insular cortex), posterior circulation (PC; thalamus, superficial posterior cerebral artery, cerebellum and brainstem), middle cerebral artery at the level of the basal ganglia (sub-MCA; M1, M2 and M3), middle cerebral artery at the level of the ventricles immediately above the basal ganglia (sup-MCA; M4, M5 and M6) and total of all regions combined using McNemar's test. Statistical significance was set at $a=0.05$. To correct for multiple comparisons, a Bonferroni correction was applied. Inter-rater reliability was evaluated using Cohen's Kappa, for which the following interpretations were used: slight (0.01 - 0.20), fair (0.21 - 0.40), moderate $(0.41-0.60)$, good $(0.61-0.80)$, near perfect (0.81 - 0.99), and perfect agreement (1). Subgroup analyses of early- and late-window patients were performed using the same methods. Statistical analyses were performed in SPSS version 25.0 (IBM, Armonk, NY, USA).

\section{Results}

\section{Patient characteristics}

One-hundred and twenty-five consecutive patients suspected of AIS were included (median age 65.0; IQR 51.8 - 80.3 years; 48\% female). In phase 1, 25 patients were included (median age 67.0; IQR 54.5 -75.3 years; 56\% female), while in phase 2, a total of 96 patients (median age 63; IQR 52.5 - 81.0 years; 46.9\% female) were included after exclusion of 4 patients for metallic artifact (1 CT case) and corrupted DWI images (3 MRI cases). Phase 1 patients had a median presenting NIHSS of 4 (IQR 1 - 11), while phase 2 patients had a median presentation NIHSS of 5 (IQR 2 - 12). Baseline patient demographics are summarized in Table 5.1. There were no significant differences in baseline patient characteristics between the groups.

\section{Phase 1: initial selection VMI energies for infarct detection}

Acute cerebral infarct was identified in 12/25 (48\%) patients by DWI. Sensitivity, specificity, PPV and NPV of each VMI reconstruction from $40 \mathrm{keV}$ to $140 \mathrm{keV}$ for the detection of infarct relative to the reference DWI are shown in Figure 5.1. The interobserver variability range for the $\mathrm{VMI}$ reconstructions was $0.34-0.75$. The $60-120 \mathrm{keV}$ reconstructions showed the highest sensitivities (25.0 - 33.3\%), but 60 - $90 \mathrm{keV}$ also had a high specificity (92.3 - 100.0\%), PPV (75.0 - 100.0\%) and NPV (57.1 - 61.9\%) with a moderate inter-reader agreement $(0.41-0.45)$. Sensitivity was lowest for the 40,50 and 130 keV VMI reconstructions (8.3 - 16.7\%), whereas the 120 and 140 keV demonstrated lower specificity versus the 60 - 90 keV reconstructions (84.6\% vs 92.3 - 100\%). Figure 5.2 illustrates typical images the observers reviewed. 
Table 5.1 | Patient characteristics of patients in phase 1 and phase 2 of the study.

\begin{tabular}{llll}
\hline Characteristics & Phase 1 $(\mathrm{n}=25)$ & Phase 2 $(\mathrm{n}=96)$ & P-value \\
\hline Sex, male:female & $11: 14$ & $51: 45$ & 0.416 \\
Age (years), median (IQR) & $67.0(54.5-75.3)$ & $63.0(52.5-81.0)$ & 1.000 \\
Presentation NIHSS, median (IQR) & $4(1-11)$ & $5(2-13)$ & 0.700 \\
Platelets, median (IQR) & $201(167-261)$ & $222(185-275)$ & 0.700 \\
Cerebral infarct on DWI, $\mathrm{n}(\%)$ & $12(48.0)$ & $51(53.1)$ & 0.648 \\
Time since last-seen-well (hours), median (IQR) & $5.0(1.4-12.0)$ & $4.2(1.9-12.0)$ & 1.000 \\
Time CT to MR (hours), median (IQR) & $5.0(3.1-11.0)$ & $5.2(3.2-12.7)$ & 0.700 \\
IV tPA administered, $\mathrm{n}(\%)$ & $4(16.0)$ & $12(12.5)$ & 0.222 \\
\hline Stroke risk factors & & & \\
\hline Coronary Artery Disease (CAD), $\mathrm{n}(\%)$ & $4(16.0)$ & $13(13.5)$ & 0.753 \\
Atrium Fibrillation, $\mathrm{n}(\%)$ & $3(12.0)$ & $12(12.5)$ & 0.946 \\
Diabetes, $\mathrm{n}(\%)$ & $7(28.0)$ & $23(24.0)$ & 0.677 \\
Hypertension, $\mathrm{n}(\%)$ & $15(60.0)$ & $55(57.3)$ & 0.807 \\
Hyperlipemia, $\mathrm{n}(\%)$ & $12(48.0)$ & $38(39.6)$ & 0.447 \\
Prior cerebrovascular incident, $\mathrm{n}(\%)$ & $3(12.0)$ & $28(29.2)$ & 0.080 \\
Prior intracranial hemorrhage, $\mathrm{n}(\%)$ & $1(4.0)$ & $10(10.4)$ & 0.320 \\
Smoker, $\mathrm{n}(\%)$ & $3(12.0)$ & $25(26.0)$ & 0.138 \\
Antiplatelet or anticoagulant, $\mathrm{n}(\%)$ & $12(48.0)$ & $47(49.0)$ & 0.932 \\
\hline
\end{tabular}

Abbreviations: IQR; interquartile range, NIHSS; National Institutes of Health Stroke Scale.
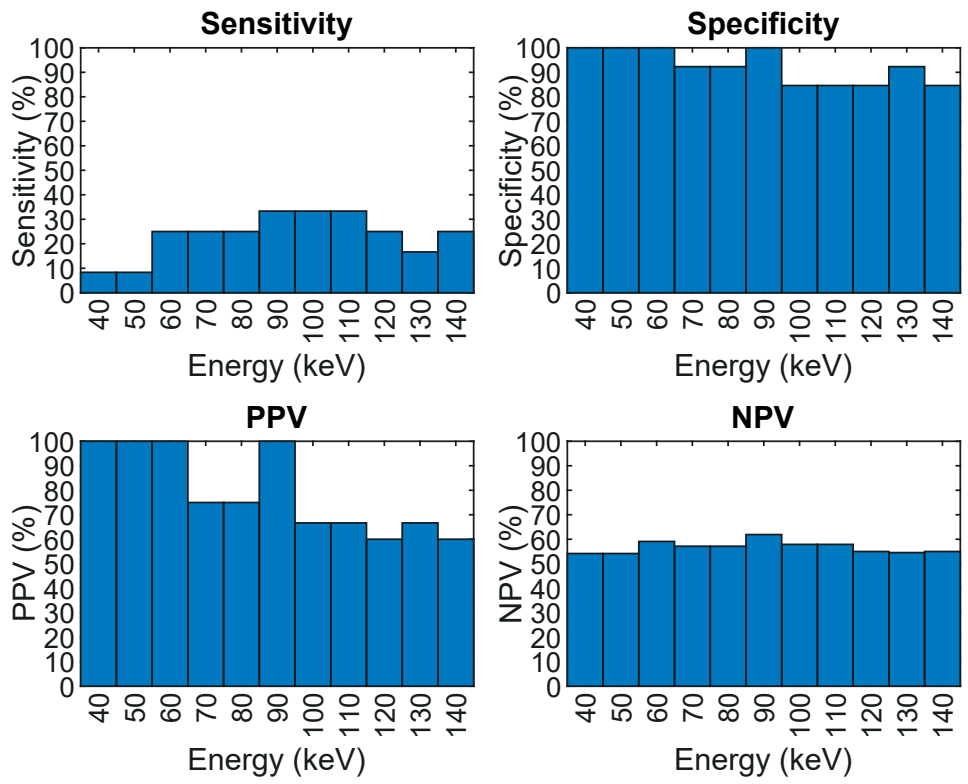

Figure 5.1 | The sensitivity, specificity, positive predictive value (PPV) and negative predictive value (NPV) (\%) of the consensus scoring of the different VMI reconstructions for detection of infarct. 


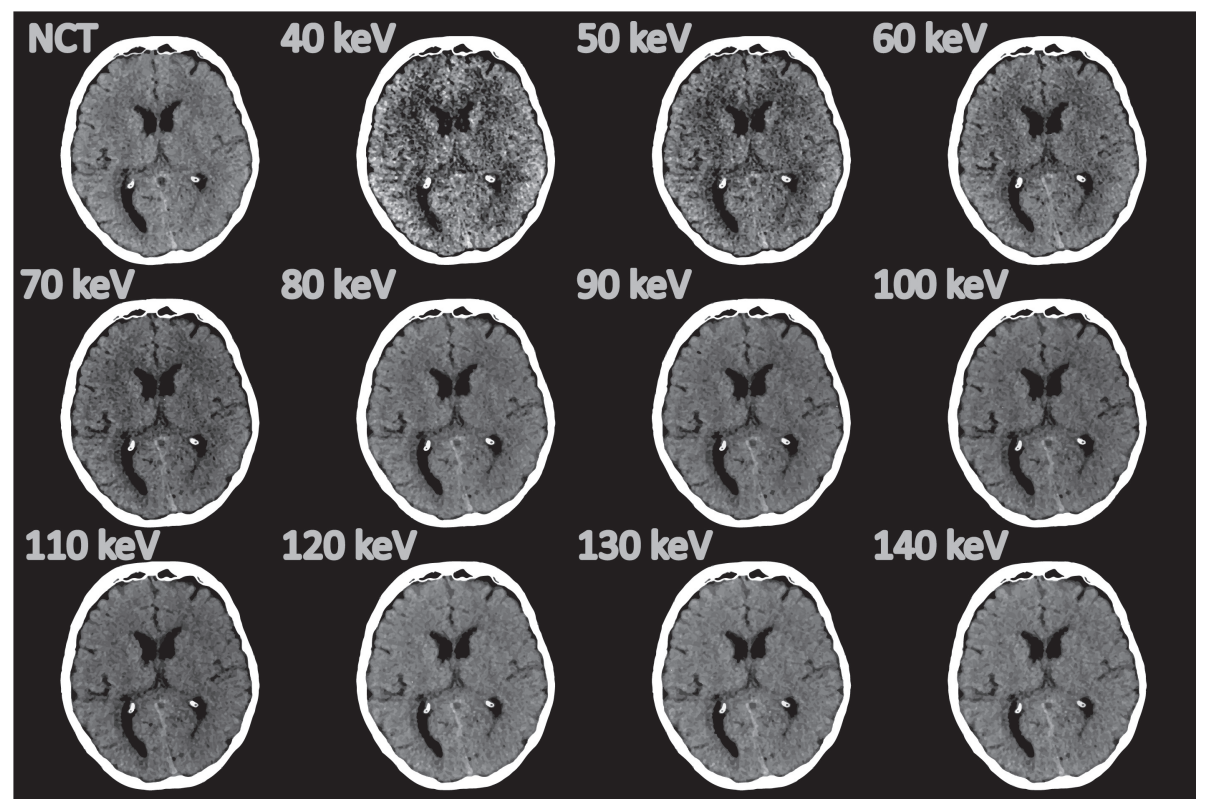

Figure $\mathbf{5 . 2}$ | Example patient of VMI and conventional reconstructions. The NCT has a WL/WW of 40/50 HU, 40 and $50 \mathrm{keV}$ (WL/WW: 50/50 HU), 60 and $70 \mathrm{keV}$ (WL/WW: 45/50 HU), 80, 90, 100 and $110 \mathrm{keV}$ (WL/WW: 40/50 HU), and 120, 130 and $140 \mathrm{keV}$ (WL/WW: 35/50 HU).

VMI reconstructions from 60 - $90 \mathrm{keV}$ were the most sensitive and specific for the detection of cerebral infarct, and were chosen for further evaluation in phase 2.

\section{Phase 2: comparison of VMI to conventional CT}

Infarct detection

DWI identified 51/96 (53\%) patients with acute infarction. The sensitivity, specificity, PPV, NPV and accuracy for the detection of infarct for all included VMI reconstructions and the NCT is shown in Table 5.2. The VMI reconstructions (70 - $90 \mathrm{keV}$ ) had higher mean sensitivity relative to NCT, even though not significant (36.0 - 37.3 vs. NCT 32.4\%; p>0.680). VMI reconstructed at 80 and 90 keV demonstrated higher sensitivity (36.0, 37.3 vs NCT 32.4\%; p>0.680), specificity (92.2, 94.4 vs NCT 91.1\%; p>0.509), PPV (84.0, 89.1 vs NCT 82.0\%), NPV (55.7, 56.6 vs NCT 54.2\%) and accuracy $(62.0,63.5$ vs NCT 59.9\%) compared to the NCT, where $90 \mathrm{keV}$ performed best. Inter-rater reliability for all reconstructions was good $(0.64-0.78)$. Figure 5.3 illustrates the difference in conspicuity of infarct between VMI and NCT in comparison to DWI. 


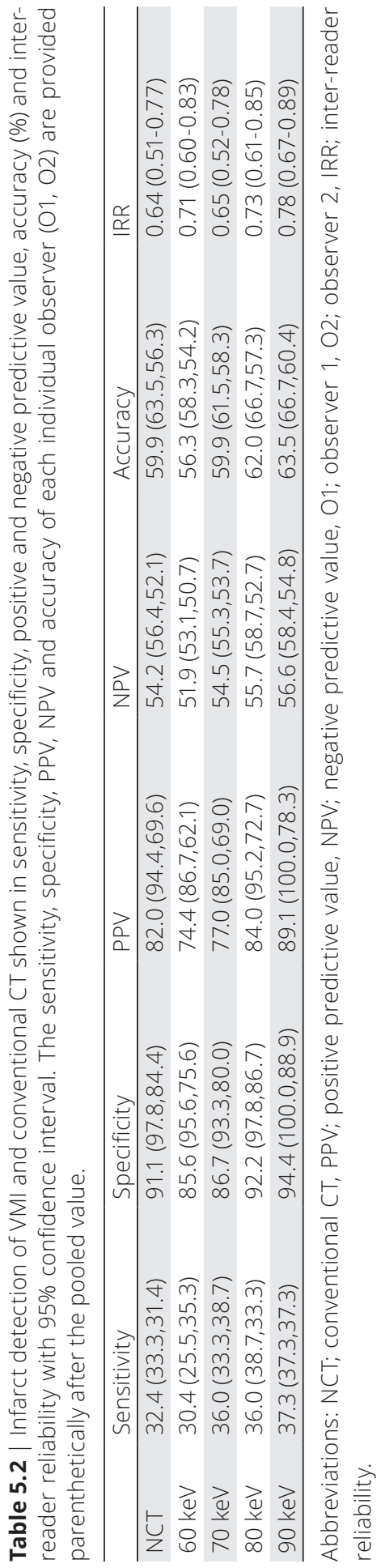




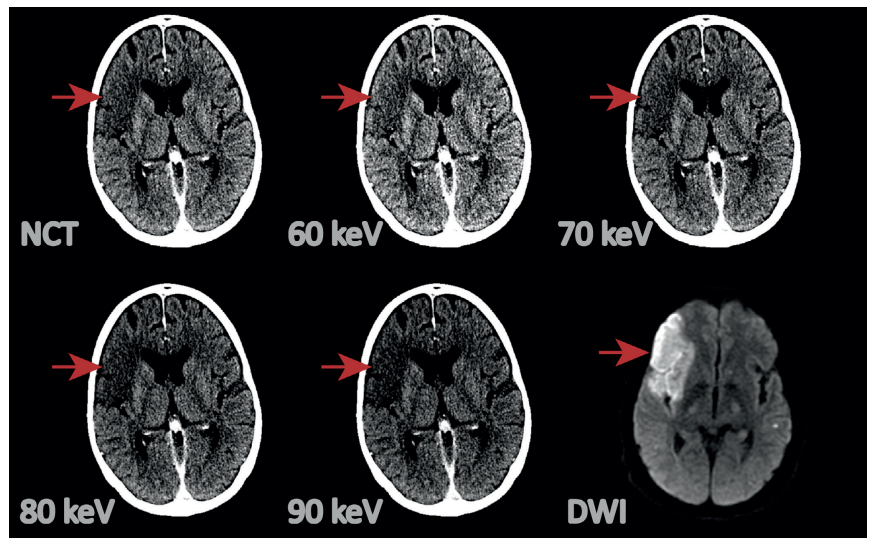

Figure 5.3 | Example case of patient demonstrating the increased conspicuity of an acute infarct on the 80 and $90 \mathrm{keV} \mathrm{VMI}$ in comparison to NCT, 60 and $70 \mathrm{keV}$ and corresponding diffusion restriction on subsequent MRI DWI confirmed the territory of infarct. Red arrows indicate the outlining of the infarcted area. The CT reconstructions have the same WL/WW: 40/30 HU.

\section{Infarct per territory}

Pooled ILM of NCT and VMI are shown in Table 5.3. NCT and VMI were unable to match the location of infarct in the anterior cerebral artery to DWI. ILM-NCT was higher in the posterior circulation (11.1 - 13.3 vs 15.6\%), though not significant. Other regions (sub-MCA, sup-MCA and basal ganglia) were higher for 80 and $90 \mathrm{keV} \mathrm{VMI} \mathrm{compared} \mathrm{to} \mathrm{NCT,} \mathrm{and} \mathrm{these}$ differences were significant in the middle cerebral artery at the level of the basal ganglia (sub-MCA; 29.6 and 33.3 vs NCT 7.4\%; p<0.015). ILM for 80 and 90 keV reconstructions for all regions was significantly higher compared to NCT (28.7 and $27.4 \%$ vs. 19.5\%; p<0.010). The inter-reader reliability was near perfect for all CT reconstructions $(0.86-0.88)$.

\section{Infarct in the early- and late-windows}

Fifty patients were scanned within the early-window (median time 1.9, IQR 0.8 - 3.0 hours). Among these patients, 25 (50.0\%) had DWI-positive acute cerebral infarct. Fortysix patients were scanned within the late-window (median time 12.0, IQR 6.3 - 17.9 hours). Among these patients, 26 (56.5\%) had DWI-positive acute cerebral infarct.

For early-window patients, VMI reconstructions at $80 \mathrm{keV}$ showed the highest mean sensitivity (26.0\%), whereas NCT had $18.0 \%$ sensitivity. The difference, however, is not significant ( $p=0.499)$. In addition, 80 keV VMI showed similar or higher specificity (90.0 vs 90.0\%; $p=1.000)$, PPV (73.8 vs 64.3\%), NPV (54.8 vs 52.3\%) and accuracy (58.0 vs 54.0\%) relative to NCT. A good inter-rater reliability was seen for all reconstructions $(0.66-0.79)$. Patients in the late-window showed higher sensitivity for 90 keV compared to NCT (50.0 vs 46.2\%; $p=0.786$ ). Specificity was higher for $90 \mathrm{keV}$ compared to NCT (95.0 vs 92.5\%; $p=0.747)$. PPV (93.3 vs 90.6\%), NPV (0.59.3 vs 56.9\%) and accuracy (69.6 vs 66.3\%) are also higher for $90 \mathrm{keV}$ compared to NCT. NCT and VMI showed good inter-rater reliability (0.61 - 0.77). Results of other VMI in early and late-window are shown in Table 5.4. 


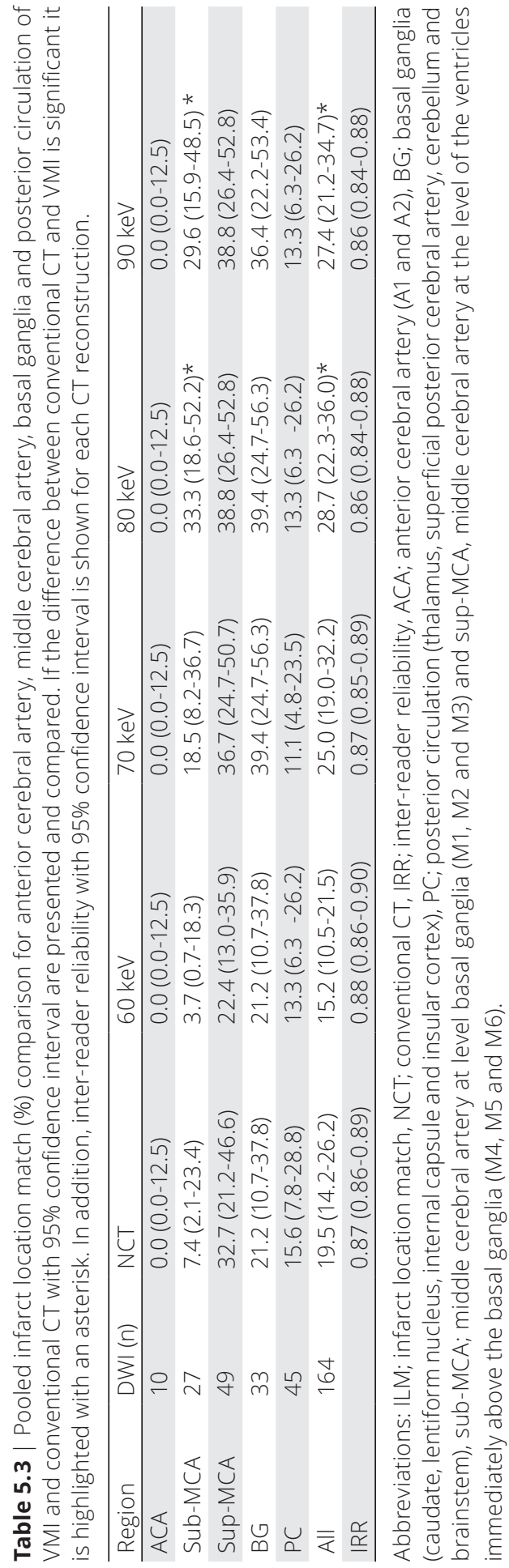


Part II | Dual-energy CT in stroke imaging

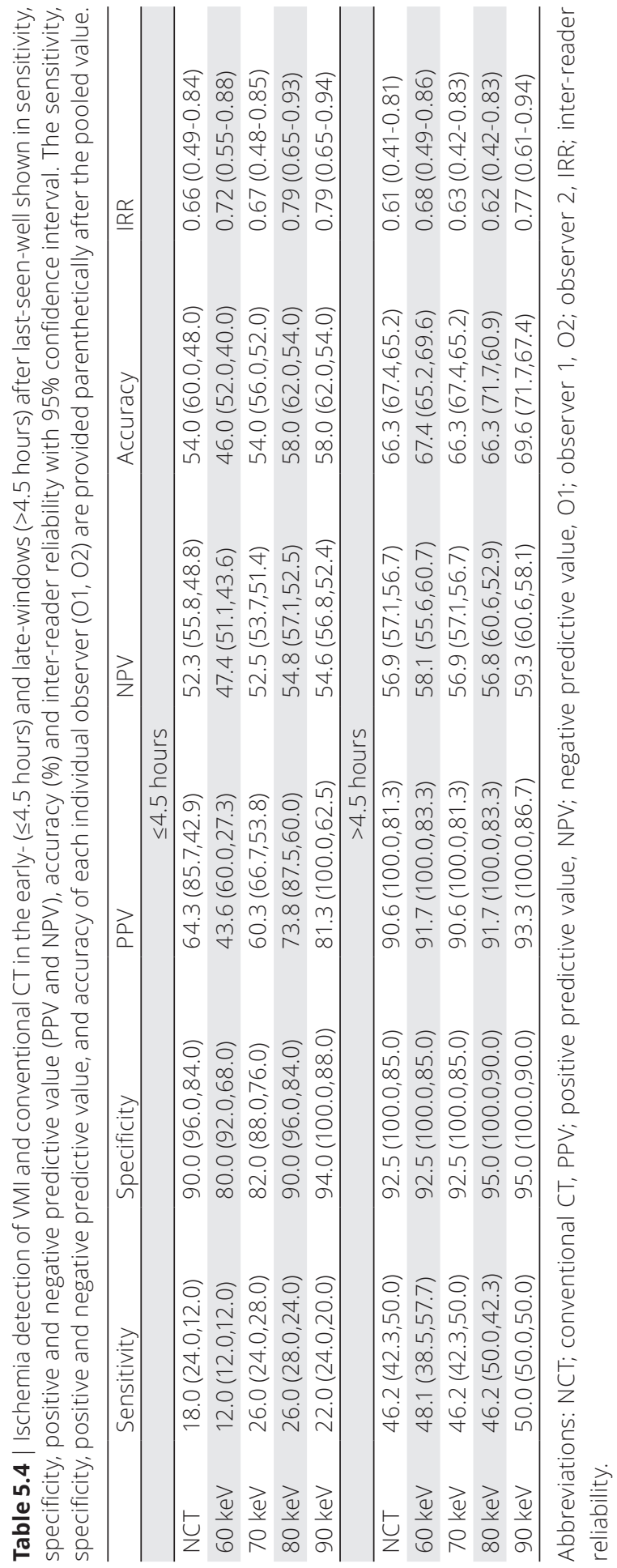


Pooled ILM of NCT and VMI in the early- and late-windows are shown in Table 5.5. In the early-window, location matching on VMI at $80 \mathrm{keV}$ was similar or better than NCT in all regions (ACA; 0.0 vs. 0.0\%, sub-MCA; 36.4 vs. 0.0\%, sup-MCA; 43.8 vs. $25.0 \%$, BG; 36.4 vs. $18.2 \%$ and $P C ; 5.9$ vs. 5.9\%). The increase, however, was not significant in any of the regions. ILM of all regions of $80 \mathrm{keV}$ VMI (27.1\%) was significantly higher $(p=0.012)$ compared to total ILM of NCT (11.9\%). The inter-reader reliability was near perfect for all CT reconstructions $(0.83-0.85)$. In the late-window, VMI at $90 \mathrm{keV}$ outperformed NCT for all territories (ACA; 0.0 vs. 0.0\%, sub-MCA; 37.5 vs. $12.5 \%$, sup-MCA; 39.4 vs. $36.4 \%$ and $B G ; 40.9$ vs. 22.7\%), except in the posterior circulation (PC; 17.9 vs. 21.4\%). The differences were not significant. The ILM of all regions of $90 \mathrm{keV}$ VMI (31.4\%) was significantly higher compared to NCT-ILM of all regions (23.8\%; $p=0.031)$. The interreader reliability was near perfect for all CT reconstructions $(0.88-0.89)$.

\section{Discussion}

In this study, VMI reconstructions at $90 \mathrm{keV}$ showed higher sensitivity (36.3\%) and specificity (94.4\%) for the detection of acute cerebral infarct in AIS patients than NCT (32.4\% and $91.1 \%$, respectively). This VMI energy is concordant with prior reports suggesting optimal VMI for maximum contrast resolution is between $60-100 \mathrm{keV}$ in adults depending on the DECT approach[23,43,95]. Pomerantz et al.[23] suggested 65 - 75 keV for overall parenchymal contrast resolution, with agreement by Neuhaus et al.[43] at 65 keV for gray-white differentiation, whereas Yoshida et al.[95] suggested that $99 \mathrm{keV}$ offered superior evaluation of supratentorial acute infarct. Yoshida et al., however, only used confirmed infarct patients, we included AIS patients. By comparing our CT findings to DWI we were able to identify the best VMI energies for differentiating between normal and infarcted tissue. Additionally, VMI reconstructions at $90 \mathrm{keV}$ also had the highest NPV (56.6 vs 54.2\%). As CT is often used as a screening modality in cases where there is concern for AIS, improvements in the sensitivity and NPV offered by $\mathrm{VMI}$ may lend confidence to the neuroradiologist in excluding cerebral infarct, particularly if MRI is contraindicated or unavailable.

Subgroup analyses of patients imaged $\leq 4.5$ hours and $>4.5$ hours from last-seen-well was performed as infarct conspicuity changes with increasing edema in the maturing lesion. Analysis of patients presenting $\leq 4.5$ hours from last-seen-well demonstrated a small improvement in sensitivity ( 26.0 vs $18.0 \%$ ) and NPV (54.8 vs $52.3 \%$ ), and similar specificity (90.0\%) for the detection of acute cerebral infarct using VMI at $80 \mathrm{keV}$ as compared to NCT. Given that patients presenting within this window are considered eligible for intravenous thrombolytic therapy, even small improvements in infarct detection may increase clinical and therapeutic certainty[10]. 
Part II | Dual-energy CT in stroke imaging

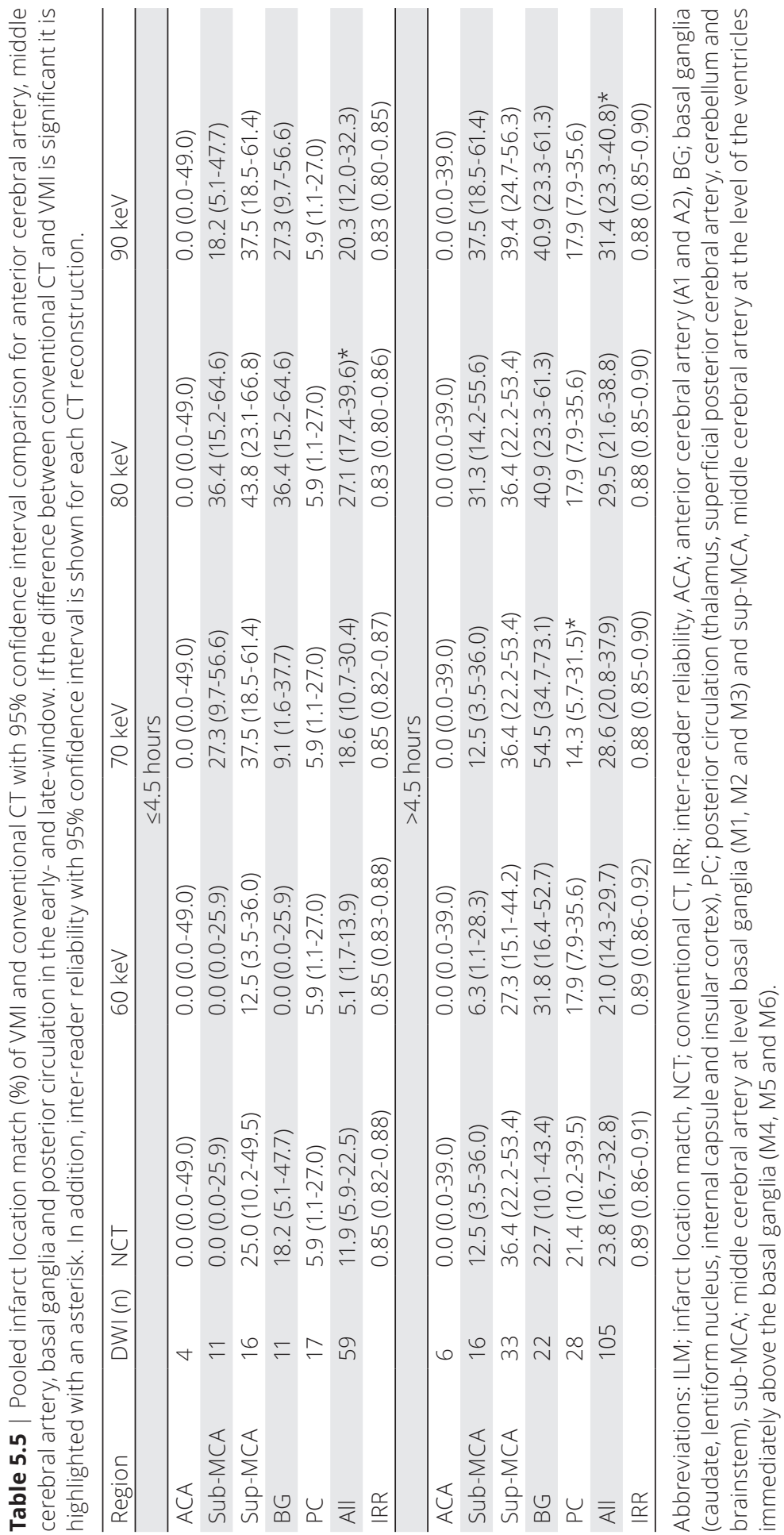


Infarct localization relative to reference standard DWI was assessed using ILM for each pre-defined cerebral region. Within the middle cerebral artery territories (subMCA and sup-MCA), a non-significant trend towards superior VMI-ILM at $80-90 \mathrm{keV}$ was observed relative to NCT-ILM. However, overall VMI-ILM showed a significant improvement with 80 - $90 \mathrm{keV}$ in comparison to total NCT-ILM (28.7, 27.4 vs 19.5\%), which suggests that VMI may significantly increase the accuracy of infarct localization. Given regional improvement in infarct detection of VMI seen at $80 \mathrm{keV}$ in the earlywindow within the anterior circulation, assessment of VMI in an anterior large-vessel occlusion population is warranted. To determine if infarct core may be better estimated with VMI than with NCT and could be used for early-window treatment decisions[96]. In our study, we see a clear trend towards a significant increase in ILM using VMI, however, our study was underpowered given the number of territories surveyed, and further testing in a larger cohort is warranted.

Relative to NCT, VMI reconstructions at 80 - 90 keV showed a significant improvement of acute infarct detection. This improvement is most likely due to the qualitatively demonstrated improved soft tissue contrast. This improved contrast therefore increases small inherent attenuation differences between gray matter, white matter and edematous tissue compared to NCT. We hypothesize that the increase in contrast is due to increased differences between attenuation of gray and white matter and to the lower noise levels in 80 and $90 \mathrm{keV}$ VMI[80,95]. The range of best VMI energies is 80 $90 \mathrm{keV}$, and we recommend individual institutions to test their own optima, but $90 \mathrm{keV}$ generally has a slightly better sensitivity and specificity for infarct detection compared to $80 \mathrm{keV}$. Infarct location detection is slightly better with $80 \mathrm{keV}$. However, if dual-energy $\mathrm{CT}$ is unavailable, similar results are unlikely to be obtained by modifying single-energy $\mathrm{CT}$ protocols to an average energy of $90 \mathrm{keV}$, as this will involve an increase in peak tube voltage and a substantial decrease in exposure to maintain a similar dose level. This increase in $\mathrm{kVp}$ will result in a decrease in contrast, because there will be a large number of photons with an energy higher than $90 \mathrm{keV}$ which will not attenuate in tissue.

Our study has several limitations. First, our retrospective study design may introduce bias. The small sample size may limit our statistical analysis, particularly for analysis of ILM. Third, our sensitivity analysis may be artificially increased as the imaging reviewers were aware that the testing population was enriched for patients with cerebral infarct. Fourth, a recognition bias can be assumed, resulting in an underestimation of the differences between the reconstruction types. We, however, blinded our reviewers to reconstruction type, CT reconstructions were presented in a random fashion and the large amount of CT reconstructions in the comparison ensured that no single energy level was favored over the other. 
Part II | Dual-energy CT in stroke imaging

Lastly, we investigated the applicability of VMI using a single type of DECT acquisition (dual-source CT scanner). DECT acquisitions, VMI techniques and reconstruction algorithms vary between manufacturers and types of DECT scanners. As a result, monochromatic energy levels cannot be reproduced between manufacturers or post processing algorithms, thereby limiting study generalizability and indicating that different institutions may need to determine VMI optima independently[79].

In conclusion, use of virtual monochromatic images at 80 - $90 \mathrm{keV}$ with non-contrast dual-energy CT results in a modest improvement of sensitivity and specificity for the detection of acute infarcts in suspected ischemic stroke patients relative to conventional non-contrast CT. 
Virtual monochromatic images for stroke detection | Chapter $\mathbf{5}$ 



\title{
CHAPTER 6
}

\section{NON-CONTRAST BRAIN CT VIRTUAL ISCHEMIA MAPS FOR CORE INFARCT VOLUME ESTIMATION}

\author{
Based on
}

DN Wolman, Fvan Ommen, E Tong, F Kauw, JW Dankbaar, E Bennink, HWAM de Jong, L Molvin, M Wintermark and JJ Heit. Dual-energy CT virtual ischemia maps improve estimation of core infarct size. Submitted.

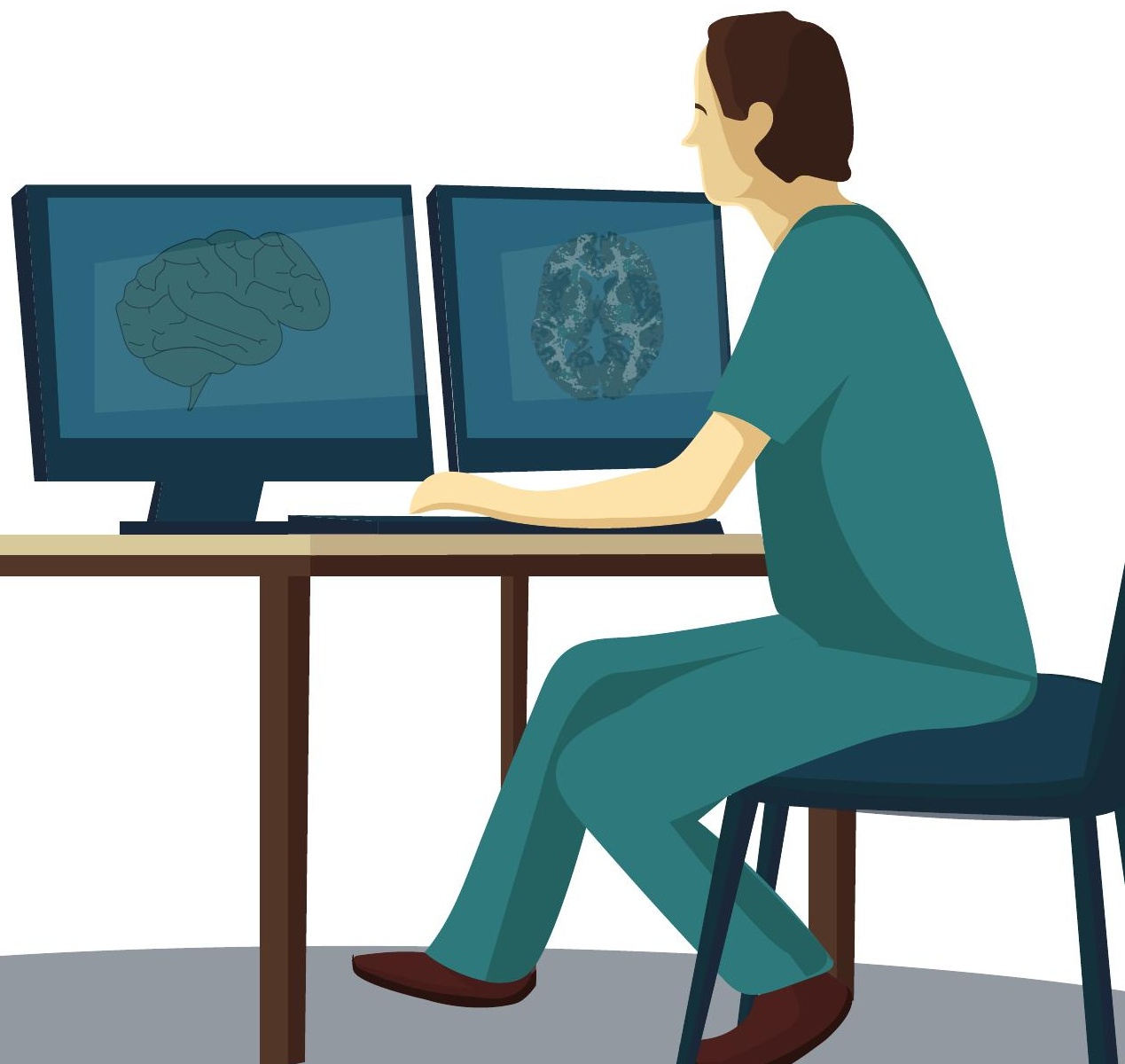


Part II | Dual-energy CT in stroke imaging

\section{Abstract \\ Objectives}

Dual-energy CT (DECT) material decomposition techniques may better detect edema within cerebral infarcts than conventional non-contrast CT (NCCT). We determined if virtual ischemia maps (VIMs) derived from non-contrast DECT of patients with acute ischemic stroke due to large-vessel occlusion (AIS-LVO) are superior to NCCT for core infarct estimation.

\section{Methods}

Consecutive AIS-LVO patients with baseline non-contrast DECT, CT perfusion (CTP), and DWI-MRI were identified between July 2018 and August 2019 from a prospective database. Patients whose baseline core infarcts were likely to remain stable on follow-up MRI were included, defined as those with excellent post-thrombectomy revascularization or no perfusion mismatch. Core infarct volumes were manually segmented on NCCT, VIM, and DWI, and derived from CTP. Core volume agreement was assessed using Bland-Altman plots and comparison of CT to DWI volume ratios. Three blinded neuroradiologists provided an Alberta Stroke Program Early CT Score (ASPECTS) for VIM and NCCT, which were compared against a consensus DWI-ASPECTS.

\section{Results}

Twenty-four patients were included (46\% female, $64.3 \pm 15.0$ years). Median time from last-seen-well to CT was 7.5 (IQR 1.5 - 14.5) hours. Final DWI volume was better approximated by VIM than NCCT $(0.68 \pm 0.35$ vs. $0.34 \pm 0.35 ; p<0.001)$ or CTP $(0.45$ $\pm 0.67 ; p<0.001)$. Bland-Altman analyses indicated significantly greater agreement between DWI and VIM than NCCT core volumes (mean bias 0.60 [95\% agreement interval 0.39 - 0.82] vs. 0.20 [95\% agreement interval $0.11-0.30]$ ). Pooled VIM- and NCCT-ASPECTS differed significantly (6.4 \pm 2.5 vs. $7.6 \pm 2.0 ; p<0.001)$, and VIM was more accurate than NCCT in estimating DWI-ASPECTS (71\% vs. 65\%).

\section{Conclusions}

DECT VIM estimates core infarction in AIS-LVO patients more accurately than NCCT. 


\section{Introduction}

Dual-energy computed tomography (DECT) scanners acquire CT images at two different energies, which allows for improved contrast resolution, image noise and beam hardening artifact reduction, and separation of tissue components, such as water, iodine, and fat[21,87]. DECT techniques have been used following acute ischemic stroke due to large-vessel occlusion (AIS-LVO) patient treatment by endovascular thrombectomy (ET) to differentiate normal post-procedural iodine contrast presence from post-ET intracranial hemorrhage and to improve the visualization of cerebral ischemia when obscured by residual parenchymal contrast opacification[26,90,97]. Previously reported custom DECT post-processing algorithms have suggested improved detection of cerebral ischemia by highlighting areas of cytotoxic edema after material separation using basis pairs of fat and calcium, or air and water, however these techniques have been technically limited and complex to interpret, or were not compared against reference standard DWI images[89,91]. As accurate core infarct delineation is critical in the triage of AIS-LVO patients to ET, CT techniques capable of superior core estimation are of high clinical relevance[14,98,99].

We hypothesized that post-processing of non-contrast DECT using an automated material decomposition algorithm to generate virtual ischemia maps (VIMs) may more accurately identify and estimate core infarction in AIS-LVO patients compared to conventional NCCT and CTP techniques. Relative to DWI-derived final core infarct volumes, we evaluated the accuracy of DECT-derived brain VIM in delineating core infarct volume and Alberta Stroke Program Early CT Scores (ASPECTS) on baseline imaging of AIS-LVO patients undergoing ET triage.

\section{Materials and Methods \\ Patients}

This study complied with the Health Insurance Portability and Accountability Act and was approved by the Stanford University institutional review board, which waived the need for written informed consent. We performed a retrospective cohort review of consecutive AIS-LVO patients who underwent DECT imaging triage for ET from July 2018 to August 2019. Patient demographic and treatment data were identified from a prospectively maintained AIS-LVO database at our comprehensive stroke center. A majority of AIS-LVO patients at our institution arrive by transfer from spoke hospitals and undergo immediate pre-procedural MRI, while AIS patients presenting to our emergency department or those who suffer a stroke while in our hospital undergo $\mathrm{CT}$ at the nearest available scanner. As such, not all patients undergo imaging at a DECT capable scanner, and technologists are instructed to perform single-energy CT if patient body habitus or motion are suspected to limit image quality on our institutional dual-source DECT. 
Inclusion criteria were: baseline DECT, age $\geq 18$ years, presentation within 24 hours of symptom onset, anterior circulation large vessel occlusion (ICA, M1- or proximal M2segment of the middle cerebral artery), and follow-up MRI with DWI within 24 hours of baseline DECT. To minimize errors in core infarct estimation between baseline DECT and follow-up reference standard DWI, only patients with matched perfusion-core infarct profiles on baseline imaging or those with successful revascularization (Thrombolysis in Cerebral Ischemia [TICI] grade 2b, 2c, or 3) after ET were included[14,100-102].

Exclusion criteria included technical failure of perfusion imaging, excessive motion artifact, and absence of core infarction on follow-up MRI. The patient selection workflow and reasons for exclusion are summarized in Figure 6.1.

\section{Imaging technical details}

All patients underwent DECT on a dual-source Somatom Definition Flash (Siemens Healthineers, Erlangen, Germany). The CT protocol included a non-contrast head CT, CT perfusion (CTP), and CT angiography of the head and neck. All DECT protocols were developed to maintain strict dose neutrality with prior conventional CT protocols at our institution. Scan parameters were: tube voltages of $80 \mathrm{kVp}$ and Sn140 kVp, tube currents of 640 and $320 \mathrm{mAs}$, pitch 1.0, beam collimation $40 \times 0.6 \mathrm{~mm}$, rotation time 1.0 s, matrix size 512×512, and slice thickness $3.0 \mathrm{~mm}$. Images were reconstructed using a medium smoothing Q34s kernel.

DECT VIM images were generated from non-contrast source data in Syngo.via (Siemens Healthineers, Erlangen, Germany) using the Brain Hemorrhage module normally used to generate virtual non-contrast images, with the following manufacturer default material definitions: Low Energy: Hemorrhage 80 HU, CSF 5 HU; High Energy: hemorrhage 75 $\mathrm{HU}, \mathrm{CSF} O \mathrm{HU}$; and relative CM 2.0. Algorithm parameters included: minimum and maximum thresholds $-20 \mathrm{HU}$ and $3071 \mathrm{HU}, \mathrm{CM}$ cutoff -50 HU, and resolution 2. Resolution enhancement, organ contour enhancement, and iodine beam hardening correction were enabled.

CTP was performed as previously described and CTP-derived core infarct volume was automatically calculated using RAPID (iSchemaView, Menlo Park, CA, USA), which identifies the core infarction as a 70\% reduction in cerebral blood flow (CBF) relative to the contralateral normal hemisphere[103].

DWI-MRI was performed for reference standard core infarct assessment, using either a 1.5T GE Signa or 3.0T GE MR750 MRI scanner with an eight-channel GE HR brain coil (GE Healthcare, Milwaukee, WI, USA). The axial DWI imaging parameters included: TR 6000 ms, TE 78.2 ms, b-values: 0 and 1000, flip angle 90, and 5 mm slice thickness. 


\section{Image analysis}

Anonymized neuroimaging data were placed into an Osirix database (Pixmeo, Bernex, Switzerland) for analysis. Imaging readers had full control in modifying window width and level for all images and were blinded to clinical, thrombectomy, and MRI data.

Core infarct volumes were manually segmented as regions of interest (ROIs) on VIM, NCCT, and DWI images by DNW, a neuroradiology fellow, and verified by JJH, a neuroradiologist with 7-years of experience. CT regions of pathological parenchymal hypoattenuation were segmented, which required loss of gray-white matter differentiation, an absence of local volume loss, and conformation to an anterior circulation vascular territory. DWI regions of infarction were segmented on the basis of hyperintensity on high b-value images. Volumes were calculated from manual ROI segmentation using a custom Osirix plug-in[104].

Alberta Stroke Program Early CT Score (ASPECTS) was assigned for all studies by three readers (JJH, DNW and ET; a neuroradiologist with 4-years of experience), each provided with baseline VIM images followed by baseline conventional NCCT images after a 6-week interval to minimize recall bias. Consensus DWI-ASPECTS, determined after an additional 6-week interval by the same readers, was used as the reference standard.

\section{Statistical analysis}

The primary outcome measure was agreement between VIM, NCCT, and CTP core infarct volumes compared to the reference standard DWI volumes. Secondary outcome measures were the accuracy of VIM- and NCCT-ASPECTS relative to DWI-ASPECTS, and inter-reader agreement for the ASPECTS analysis. Both per-reader and pooled analyses were performed.

Pairwise comparisons of all core infarct volumes derived from VIM, NCCT, CTP, and DWI were performed using the Mann-Whitney $U$ test, and ASPECTS comparisons were performed using the Wilcoxon signed-rank test. Normalized core infarct volumes were calculated as the fraction of the CT volume divided by the DWI volume. Bland-Altman analyses were used to assess volume and ASPECTS agreement between NCCT, VIM, and reference DWI, both before and after logarithmic correction of non-Gaussian data[105,106]. The Shapiro-Wilk test was performed using the Royston method to assess for volume distribution normality, and a logarithmic regression of the data was performed to correct for non-Gaussian distributions in Bland-Altman plots. Mean bias values and 95\% agreement intervals were reported, with significant differences between data sets determined by absence of overlap between the agreement intervals. No p-values are provided for Bland-Altman analyses as they may inadequately reflect clinically meaningful differences between measurement methods or affirm spurious correlations[105]. 
Spearman-rank correlation coefficients were calculated for comparisons of ASPECTS and core volumes. Inter-reader reliability for VIM- and NCCT-ASPECTS was assessed using Fleiss' $k$ test and the intraclass correlation coefficient (ICC).

Subgroup analyses of core infarct volume and ASPECTS agreement between VIM, NCCT, and DWI were performed as a function of time from last-seen-well (LSW) to CT imaging. Patients imaged within the early-window ( $\leq 6$ hours) were compared against those in the late-window ( $>6$ hours) to determine if VIM performance varied between windows. Data were analyzed in MATLAB (MathWorks, Natick, MA, USA) and SPSS version 25.0 (IBM, Armonk, NY, USA) by FvO and DNW. Statistical significance was set at $a=0.05$.

\section{Results}

\section{Baseline patient characteristics}

Twenty-four patients (46\% female, $64.3 \pm 15.0$ years) who underwent DECT triage for AIS-LVO were included (Figure 6.1).

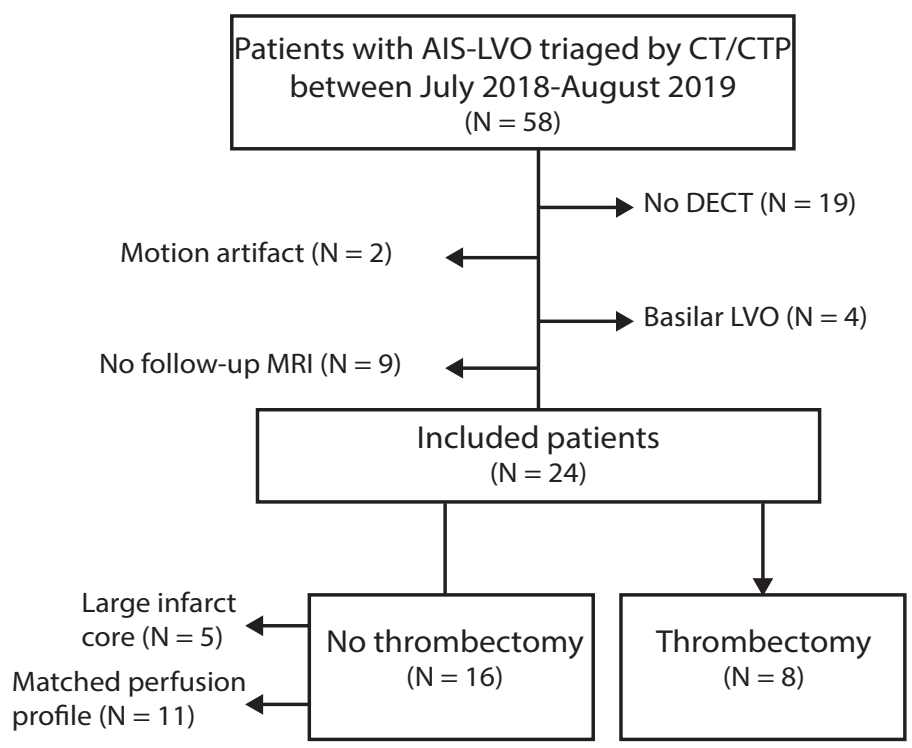

Figure 6.1 | Patient selection flowchart demonstrating the method of patient inclusion and exclusion. Consecutive patients undergoing CT and CTP triage with AIS-LVO between July 2018 and August 2019 were included, with exclusion of patients with posterior circulation occlusions, those with significantly motion degraded examinations, absent follow-up MRI, or studies performed without dual-energy mode activated. Patients were excluded from subsequent thrombectomy if the region of core infarction was large $(\geq 70 \mathrm{ml})$ or matched to the region of perfusion deficit (matched perfusion profile). 
Patient demographic data and baseline imaging characteristics are summarized in Table 6.1. LVO were localized to: ICA (5/24; 21\%), M1 segment of the MCA $(11 / 24 ; 46 \%)$, tandem ICA-M1 segment (2/24; 8\%), and proximal M2 segment of the MCA (6/24; $25 \%)$. The median time from LSW to CT was 7.5 (IQR 1.5 - 14.5) hours, and the median time from CT to MRI was 9.1 (IQR 2.3 - 14.5) hours. Eleven patients (46\%) presented in the early time window and underwent DECT $\leq 6$ hours from last-seen-well. The median presentation National Institutes of Health Stroke Scale (NIHSS) was 16 (IQR 7 - 20). Eight patients (33\%) underwent emergent ET with all achieving TICI 2b (1/8; 13\%), TICI 2c (3/12; 38\%), or complete (TICI 3 [4/8; 50\%]) revascularization.

Table 6.1 | Baseline patient demographics, imaging and treatment characteristics, with additional dichotomization by time from symptom onset to presentation, defined as the early- ( $\leq 6$ hours) and late-window ( $>6$ hours).

\begin{tabular}{|c|c|c|c|}
\hline Characteristics & $\begin{array}{l}\text { All Patients } \\
(n=24)\end{array}$ & $\begin{array}{l}\text { Early-window } \\
(n=11)\end{array}$ & $\begin{array}{l}\text { Late-window } \\
(n=13)\end{array}$ \\
\hline Sex, male:female & $13: 11$ & $4: 7$ & $9: 4$ \\
\hline Age (years), mean \pm SD & $64.3 \pm 15.0$ & $65.8 \pm 15.6$ & $63.0 \pm 15.0$ \\
\hline Presentation NIHSS, median (IQR) & $16(7-20)$ & $19(11-20)$ & $11(7-19)$ \\
\hline \multicolumn{4}{|l|}{ LVO location } \\
\hline ICA, n (\%) & $5(21)$ & $4(36)$ & $1(8)$ \\
\hline M1, n (\%) & $11(46)$ & $4(36)$ & $7(54)$ \\
\hline Tandem ICA-M1, n (\%) & $2(8)$ & $1(9)$ & $1(8)$ \\
\hline Proximal M2, n (\%) & $6(25)$ & $2(18)$ & $4(31)$ \\
\hline $\begin{array}{l}\text { Time since last-seen-well (hours), median } \\
\text { (IQR) }\end{array}$ & $7.5(1.5-14.5)$ & $1.5(0.9-2.4)$ & $14.1(9.7-17.2)$ \\
\hline Time CT to MR (hours), median (IQR) & $9.1(2.3-14.5)$ & $7.0(1.4-11.4)$ & $12.2(5.4-15.4$ \\
\hline Thrombectomy, n (\%) & $8(33)$ & $3(27)$ & $5(38)$ \\
\hline TICI 0-2a, n (\%) & $0 / 8(0)$ & $0 / 3(0)$ & $0 / 5(0)$ \\
\hline $\mathrm{TICl} 2 \mathrm{~b}, \mathrm{n}(\%)$ & $1 / 8(13)$ & $0 / 3(0)$ & $1 / 5(20)$ \\
\hline $\mathrm{TICl} 2 \mathrm{C}, \mathrm{n}(\%)$ & $3 / 8(38)$ & $1 / 3(33)$ & $2 / 5(40)$ \\
\hline $\mathrm{TICl} 3, \mathrm{n}(\%)$ & $4 / 8(50)$ & $2 / 3(66)$ & $2 / 5(40)$ \\
\hline $\begin{array}{l}\text { Time last-seen-well to revascularization } \\
\text { (hours), mean } \pm S D\end{array}$ & $9.6 \pm 6.6$ & $3.0 \pm 2.1$ & $13.6 \pm 4.5$ \\
\hline $\begin{array}{l}\text { Time CT to revascularization (hours), mean } \\
\pm \text { SD }\end{array}$ & $1.5 \pm 1.0$ & $1.6 \pm 1.5$ & $1.5 \pm 0.9$ \\
\hline
\end{tabular}

Abbreviations: SD; standard deviation, IQR; interquartile range, NIHSS; National Institutes of Health Stroke Scale.

\section{Quantitative core infarct volume assessment}

Core infarct conspicuity prior to ET was compared between VIM, NCCT, and CTP (Figure 6.2 and Table 6.2). 


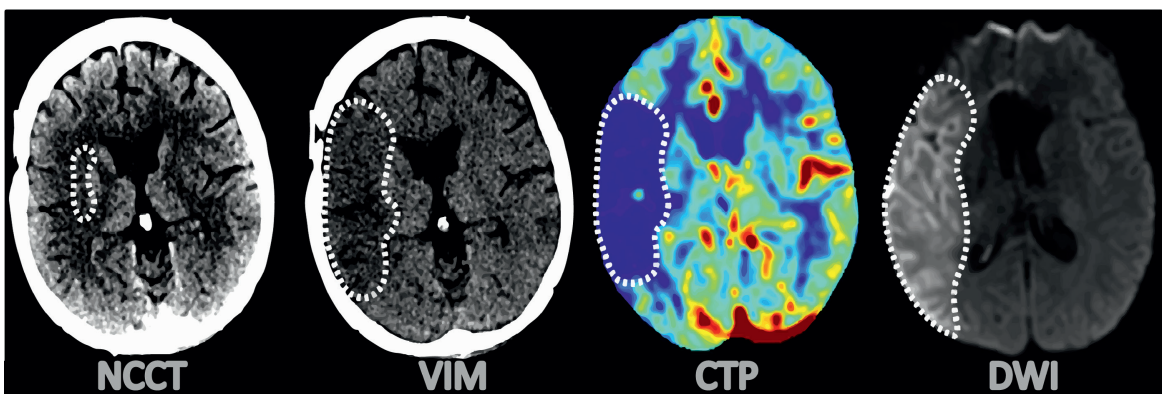

Figure 6.2 | Example case of a 49-year old female presenting in the early-window with an occlusion of the proximal right M1 segment of the middle cerebral artery and a large core infarct. Axial non-contrast CT (NCCT), virtual ischemia map (VIM), CT perfusion cerebral blood flow map (CTP) and diffusion-weighted MRI (DWI) images are presented. Note the increased conspicuity of core infarction on the axial non-contrast VIM series relative to the NCCT, using the same window width $(40 \mathrm{HU})$ and level $(25 \mathrm{HU})$ settings for each image. Within the same territory, reduced CBF and corresponding diffusion restriction on subsequent MRI confirm the territory of infarction.

Normalized VIM core volumes more closely approximated DWI core volumes when compared to conventional NCCT $(0.68 \pm 0.35$ vs. $0.34 \pm 0.35 ; p<0.001)$ or CTP core estimates $(0.45 \pm 0.67 ; p<0.001)$. Relative to DWI, VIM tended to underestimate core volumes (74.8 $\pm 103.5 \mathrm{ml}$ vs. $39.7 \pm 39.3 \mathrm{ml} ; \mathrm{p}=0.26)$. By contrast, core volumes were significantly underestimated by NCCT (18.6 $\pm 22.4 \mathrm{ml} ; \mathrm{p}=0.004)$ and CTP $(34.0 \pm 68.8 \mathrm{ml}$; $\mathrm{p}=0.003)$. Shapiro-Wilk testing demonstrated that all volume data were non-normally distributed (VIM: V=3.7, $p=0.003$; NCCT: $V=5.7, p<0.001 ; D W I: V=8.2, p<0.001$ ).

Table 6.2 | Mean \pm standard deviation core infarct and normalized core infarct volumes are presented with pairwise statistical comparisons. CTP core infarct estimates were derived by automatic segmentation of $\mathrm{CBF}<30 \%$ volumes using RAPID software.

\begin{tabular}{llll}
\hline & Core infarct volume $(\mathrm{ml})$ & $\begin{array}{l}\text { Normalized core infarct } \\
\text { volume against DWI }\end{array}$ \\
\hline Manual NCCT & & $18.6 \pm 22.4$ & $0.34 \pm 0.35$ \\
Manual VIM & $39.7 \pm 39.3$ & $0.68 \pm 0.35$ \\
Automatic CTP & $34.0 \pm 68.8$ & $0.45 \pm 0.67$ \\
Manual DWI & $74.8 \pm 103.5$ & \\
\hline Mean core infarct volume comparisons & Normalized core infarct comparisons \\
\hline \multicolumn{4}{l}{} \\
\hline VIM vs. NCCT & P-value & & P-value \\
\hline VIM vs. CTP & 0.001 & VIM vs. NCCT & $<0.001$ \\
VIM vs. DWI & 0.26 & VIM vs. CTP & $<0.001$ \\
NCCT vs. CTP & 0.19 & NCCT vs. CTP & 0.12 \\
NCCT vs. DWI & 0.004 & & \\
\hline
\end{tabular}

Abbreviations: VIM; virtual ischemia maps, NCCT; non-contrast CT, CTP; CT perfusion, DWI; diffusion-weighted imaging. 
CT core infarct volumes were compared against corresponding reference DWI volumes, and VIM best correlated with DWI $\left(\rho_{\mathrm{VIM}}=0.94 ; \rho_{\mathrm{NCCT}}=0.82 ; \rho_{\mathrm{CTP}}=0.67\right)$. Volume agreement was assessed by Bland-Altman analyses comparing normalized CT volumes against reference DWI volumes in both logarithmically corrected and uncorrected plots (Figure 6.3A and Figure 6.3B). Mean bias values for normalized and logarithmically corrected VIM volumes were 0.68 (95\% agreement interval 0.53 to 0.83 ) and -0.54 (95\% agreement interval -0.81 to -0.28), while bias values for NCCT were 0.34 (95\% agreement interval 0.21 to 0.46$)$ and -1.46 (95\% agreement interval -1.92 to -1.01$)$, respectively. The VIM core volume mean bias values were closer to agreement with the DWI volumes than the NCCT, and corresponding agreement intervals were non-overlapping.

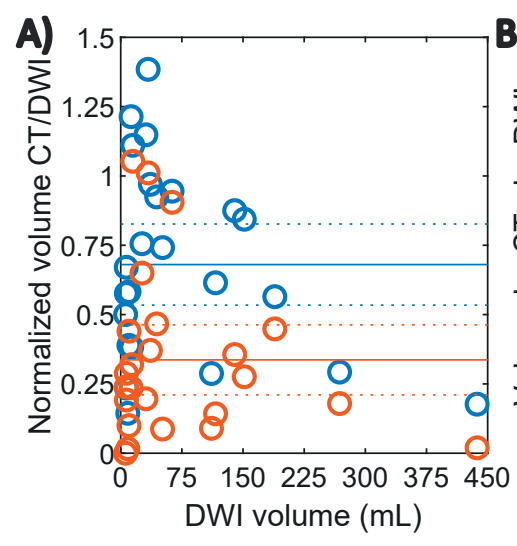

O VIM

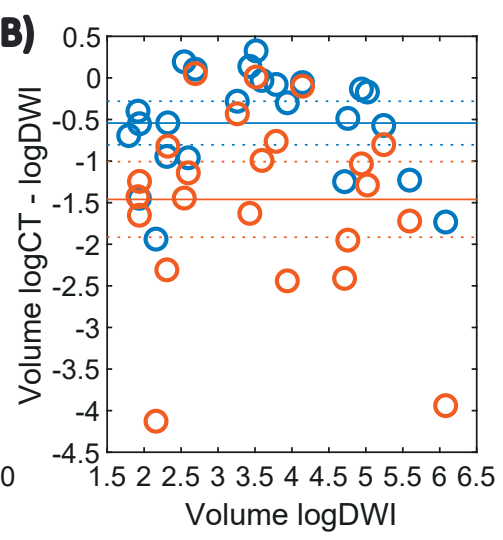

O NCCT

Figure 6.3 | Bland-Altman analyses of core infarct volumes are presented relative to reference standard DWI infarct volumes. A) VIM and NCCT volumes normalized against reference DWI volumes for all patients with solid lines indicating the mean bias value for each measurement, and hashed lines indicating the 95\% agreement interval limits. Values closer to 1.0 indicate greater agreement with the reference DWI volume. In order to correct for a non-Gaussian distribution of volume data, B) logarithmically corrected VIM and NCCT volumes minus the logarithmically corrected DWI volumes are plotted against the logarithmically corrected DWI volumes. Solid lines indicate the mean bias value for each measurement, and hashed lines indicate the 95\% agreement interval limits. Values closer to 0.0 indicate greater agreement with the reference DWI volume.

\section{ASPECTS analysis}

Pooled and individual reader ASPECTS results are shown in Table 6.3. There was a significant difference in ischemia assessment between VIM- and NCCT-ASPECTS (6.4 \pm 2.5 vs. $7.6 \pm 2.0 ; p<0.001)$. Pooled VIM-ASPECTS (6.4 \pm 2.5$)$ more closely agreed with DWI-ASPECTS ( $4.8 \pm 2.4 ; 71 \%$ accuracy) compared to NCCT-ASPECTS (7.6 $\pm 2.0 ; 65 \%$ accuracy). VIM- and NCCT-ASPECTS were plotted against corresponding DWI-ASPECTS, with a slightly stronger correlation between VIM and DWI $\left(\rho_{\mathrm{VIM}}=0.74 ; \rho_{\mathrm{NCCT}}=0.71\right.$; Figure 6.4A). 
A Bland-Altman analysis plotting the difference between CT-ASPECTS and DWI-ASPECTS was performed, with values closer to 0.0 indicating greater agreement (Figure 6.4B). Mean bias values for the difference between VIM-ASPECTS (1.58; 95\% agreement interval 0.89 to 2.28) or NCCT-ASPECTS (2.79; 95\% agreement interval 2.08 to 3.50) and DWI-ASPECTS trended toward superior accuracy with VIM; however, the agreement intervals remained overlapping. ASPECTS interrater agreement was greater for VIM $(k=0.23$; ICC $=0.64[0.43-0.81])$ than for NCCT $(k=0.17$; ICC $=0.62[0.40-0.80])$.

Table 6.3 | Pooled and individual reader ASPECTS comparisons are presented (mean \pm standard deviation), as are pooled measures of inter-reader reliability (ICC and Fleiss' k). For the individual reader ASPECTS comparisons (lower panel), each numbered p-value (1 - 3) is derived by comparing the row-designated reader's VIM-ASPECTS to the NCCT-ASPECTS of each correspondingly numbered reader (1 - 3).

\begin{tabular}{|c|c|c|c|c|c|}
\hline & & ASPECTS & P-value & Fleiss' k & ICC (IQR) \\
\hline \multicolumn{2}{|c|}{ DWI-ASPECTS } & $4.8 \pm 2.4$ & & & \\
\hline \multicolumn{2}{|c|}{ Pooled NCCT-ASPECTS } & $7.6 \pm 2.0$ & \multirow{2}{*}{$<0.001$} & 0.17 & $0.62(0.40-0.80)$ \\
\hline Pooled VII & PECTS & $6.4 \pm 2.5$ & & 0.23 & $0.64(0.43-0.81)$ \\
\hline \multicolumn{6}{|c|}{ Individual Reader ASPECTS Comparisons } \\
\hline & NCCT-ASPECTS & VIM-ASPECTS & P-value (1) & P-value (2) & P-value (3) \\
\hline Reader 1 & $7.3 \pm 2.2$ & $5.9 \pm 2.3$ & $<0.001$ & $<0.001$ & $<0.001$ \\
\hline Reader 2 & $7.6 \pm 1.9$ & $6.5 \pm 2.4$ & 0.14 & $<0.001$ & 0.001 \\
\hline Reader 3 & $8.0 \pm 2.0$ & $6.8 \pm 2.8$ & 0.42 & 0.08 & 0.002 \\
\hline
\end{tabular}

Abbreviations: VIM; virtual ischemia maps, NCCT; non-contrast CT, CTP; CT perfusion, DWI; diffusion-weighted imaging, ICC; intraclass correlation coefficient.

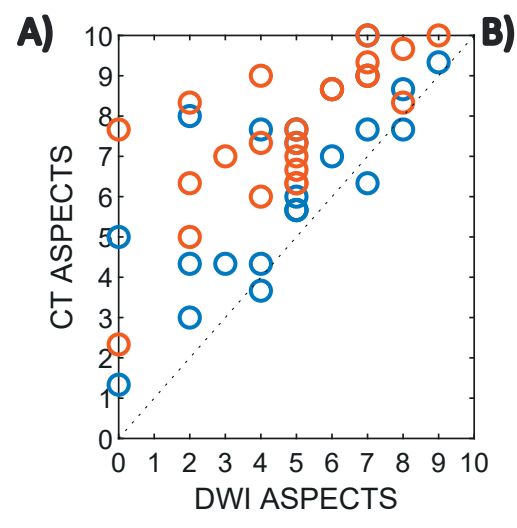

O VIM

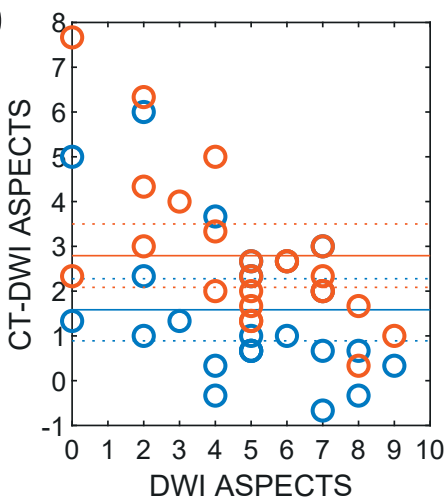

O NCCT

Figure 6.4 | Analyses of CT-ASPECTS presented relative to DWI-ASPECTS. A) VIM-ASPECTS and NCCT-ASPECTS for each patient are plotted against DWI-ASPECTS, with values falling upon the line of normality (dotted line) indicating greatest agreement to the DWI reference. B) The difference between VIM- or NCCT-ASPECTS and the DWI-ASPECTS are plotted against the corresponding DWI-ASPECTS. Solid lines indicate the mean bias value for each measurement, and hashed lines indicate the 95\% agreement interval limits. Values closer to 0.0 indicate greater agreement with the DWI-ASPECTS. 


\section{Core infarct assessment in the early- and late-windows}

The conspicuity of core infarction on CT may be influenced by the time since patients were last-seen-well. To determine if the accuracy of VIM differed depending upon LSW time, patients were dichotomized into early ( $\leq 6$ hours) and late ( $>6$ hours) time windows (Table 6.1). Forty-six percent (11/24) of patients presented early with a median LSW time of 1.5 hours (IQR $0.9-2.4$ ) and a median time from CT to MRI of 7.0 hours (IQR 1.4 - 11.4). Fifty-four percent (13/24) of patients presented late with a median LSW time of 14.1 hours (IQR 9.7 - 17.2) and a median time from CT to MRI of 12.2 hours (IQR $5.4-15.4)$.

Core infarct volume (Table 6.4) dichotomized by time window, and normalized core volume and ASPECTS agreement are presented as a function of time in Figure 6.5. Normalized VIM and NCCT core volumes (Table 6.4) significantly differed in both early- $(0.60 \pm 0.32$ vs. $0.20 \pm 0.14 ; p<0.001)$ and late-window $(0.75 \pm 0.37$ vs. $0.45 \pm$ 0.35 ; $p<0.001)$ patients; VIM core infarct assessment was more accurate in both time windows. However, in early-window patients, the difference between VIM and NCCT core estimates $(0.40 \pm 0.25)$ were $25 \%$ larger than in late-window patients ( $0.30 \pm 0.30$; $\mathrm{p}=0.22)$.

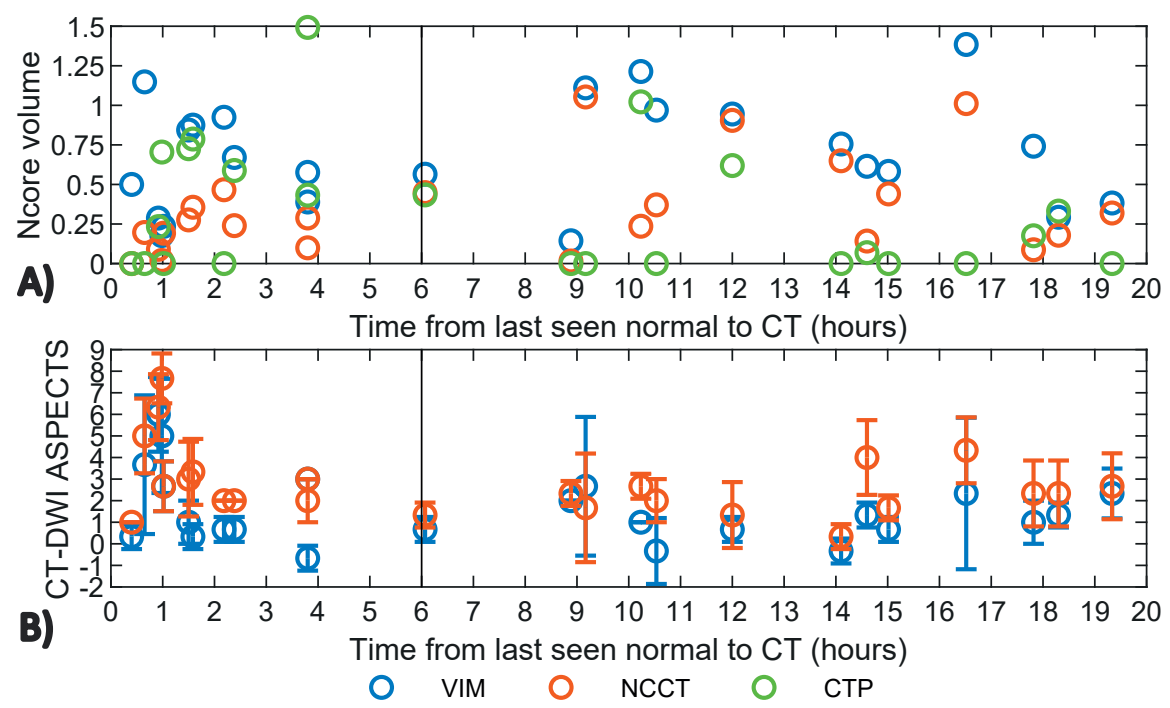

Figure 6.5 $\mid$ ASPECTS and ischemic core volume agreement as a function of time. A) Scatter plot comparing NCCT, VIM, and automatic CBF $<30 \%$ core infarct volumes normalized against manual DWI volumes (Ncore volume) for each case as a function of time since last-seen-well. Values of 1.0 indicate perfect agreement with the reference DWI volume. B) Scatter plot comparing DWIASPECTS subtracted from pooled NCCT- and VIM-ASPECTS for each case as a function of time since last-seen-well. The degree of agreement between the CT- and DWI-ASPECTS is estimated by decreasing plotted values, with a value of 0 indicating perfect agreement. A vertical line at 6 hours visually separates the early- and late-window patients. 
Part II | Dual-energy CT in stroke imaging

Table 6.4 | Data for patients presenting by time from last-seen-well to CT of $\leq 6$ hours (earlywindow) and $>6$ hours (late-window). Mean \pm standard deviation (normalized) core infarct volumes are compared, and the relative difference in normalized volume accuracy between the NCCT and VIM as a function of time is presented (normalized VIM-NCCT). P-value (vertical) reflects the pairwise statistical comparison between normalized VIM and NCCT within the same column.

\begin{tabular}{llll}
\hline & Early-window $(n=11)$ & Late-window $(n=13)$ & P-value \\
\hline Manual NCCT $(\mathrm{ml})$ & $13.0 \pm 17.4$ & $23.3 \pm 25.7$ & 0.20 \\
Manual VIM $(\mathrm{ml})$ & $41.2 \pm 47.6$ & $38.5 \pm 32.7$ & 0.64 \\
Manual DWI $(\mathrm{ml})$ & $86.5 \pm 129.2$ & $64.9 \pm 80.1$ & 0.52 \\
Normalized NCCT & $0.20 \pm 0.14$ & $0.45 \pm 0.35$ & 0.11 \\
Normalized VIM & $0.60 \pm 0.32$ & $0.75 \pm 0.37$ & 0.33 \\
P-value (vertical) & $<0.001$ & $<0.001$ & \\
Normalized VIM-NCCT & $0.40 \pm 0.25$ & $0.30 \pm 0.30$ & 0.22 \\
\hline
\end{tabular}

Abbreviations: VIM; virtual ischemia maps, NCCT; non-contrast CT, DWI; diffusion-weighted imaging.

In the early-window, Bland-Altman analyses demonstrated mean bias values for normalized and logarithmically corrected VIM volumes of 0.60 (95\% agreement interval 0.39 to 0.82 ) and -0.66 (95\% agreement interval -1.07 to -0.25$)$, while bias values for NCCT were 0.20 (95\% agreement interval 0.11 to 0.30 ) and -1.77 (95\% agreement interval -2.39 to -1.15), respectively (Figure 6.6). Early-window VIM core volume bias values indicated greater agreement with the DWI volumes than NCCT, and corresponding agreement intervals were non-overlapping. In the late-window, mean bias values for normalized and logarithmically corrected VIM volumes were 0.75 (95\% agreement interval 0.52 to 0.97 ) and -0.44 (95\% agreement interval -0.83 to -0.06), while bias values for NCCT were 0.45 (95\% agreement interval 0.23 to 0.67 ) and -1.24 (95\% agreement interval -1.95 to -0.54), respectively (Figure 6.6). Late-window VIM core volume bias values indicated a trend toward superior DWI volume estimation over NCCT, though the overlapping agreement intervals indicated a non-significant difference between methods in this subgroup. 

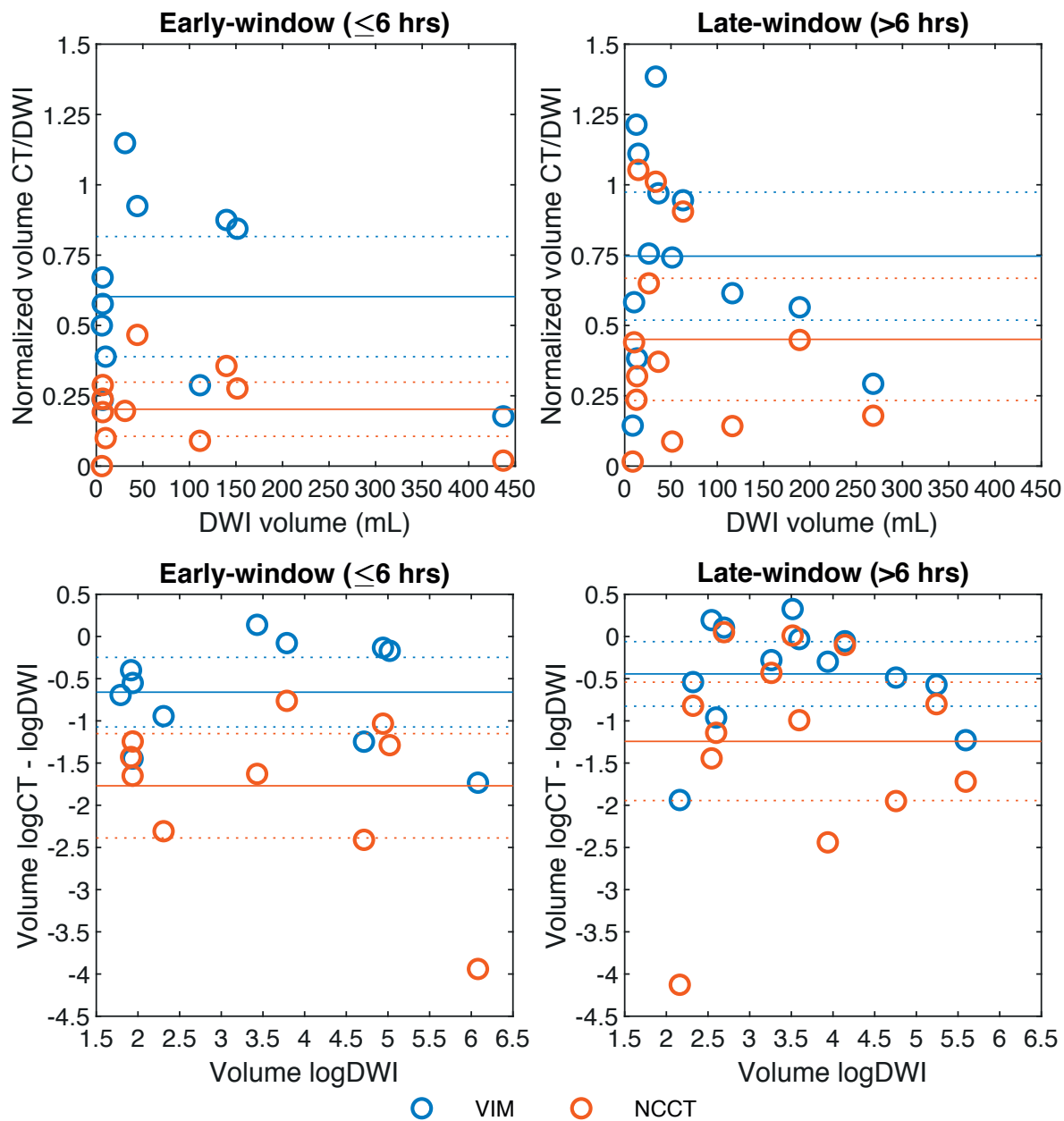

Figure 6.6 | Bland-Altman analyses of core infarct volumes are presented relative to reference standard DWI infarct volumes. VIM and NCCT volumes normalized against reference DWI volumes for patients in the early- and late-windows are plotted against the corresponding DWI volumes (top row), with solid lines indicating the mean bias value for each measurement, and hashed lines indicating the $95 \%$ agreement interval limits. Values closer to 1.0 indicate greater agreement with the reference DWI volume. In order to correct for a non-Gaussian distribution of volume data, logarithmically corrected VIM and NCCT volumes minus the logarithmically corrected DWI volumes are plotted against the logarithmically corrected DWI volumes for patients presenting in the early- and late-window, respectively (bottom row). Solid lines indicate the mean bias value for each measurement, and hashed lines indicate the 95\% agreement interval limits. Values closer to 0.0 indicate greater agreement with the reference DWI volume. 
Relative to DWI-ASPECTS $(4.8 \pm 2.7$ and $4.8 \pm 2.4)$, pooled VIM-ASPECTS was more accurate than NCCT-ASPECTS in both the early- $(6.9 \pm 2.5$ vs. $8.3 \pm 1.7 ; p<0.001)$ and latewindows ( $6.0 \pm 2.5$ vs. $7.1 \pm 2.2 ; p<0.001)$ (Table 6.5). VIM-ASPECTS was more accurate than NCCT-ASPECTS for all readers in the early-window $(p=0.004,0.03,0.02)$, but only remained significant for two of three readers in the late-window $(p=0.02,0.004$, and 0.07). Bland-Altman analyses plotting the difference between CT- and DWI-ASPECTS against DWI-ASPECTS were performed for each time-window (Figure 6.7). In both earlyand late-windows, mean bias values for the difference between VIM-ASPECTS (2.06 [95\% agreement interval 0.61 to 3.51] and 1.18 [95\% agreement interval 0.58 to 1.77]) or NCCT-ASPECTS (3.46 [95\% agreement interval 2.08 to 4.83] and 2.23 [95\% agreement interval 1.57 to 2.90]) and DWI-ASPECTS trended toward superior accuracy with VIM, however the agreement intervals remained overlapping. Interrater ICCS were similar in the early-window versus the late-window for both VIM and NCCT (ICC $=0.57$ [IQR $0.21-0.84$ ] and 0.61 [IQR $0.30-0.84$ ] vs. ICC=0.65 [IQR $0.32-0.88]$ and 0.64 [IQR $0.34-0.86])$.

Table 6.5 | Data for patients presenting by time from last-seen-well to CT of $\leq 6$ hours (earlywindow) and $>6$ hours (late-window). Pooled VIM- and NCCT-ASPECTS were compared against the DWI-ASPECTS (mean \pm standard deviation) (top panel) as a function of time. Individual reader ASPECTS (mean \pm standard deviation) were compared in a pairwise fashion, with each numbered p-value representing a comparison between the given readers VIM-ASPECTS and the NCCTASPECTS for readers 1, 2, and 3, respectively (bottom panel). P-value (vertical) reflects the pairwise statistical comparison between pooled VIM- and NCCT-ASPECTS within the same column.

\begin{tabular}{|c|c|c|c|c|c|}
\hline & \multicolumn{2}{|c|}{ Early-window $(n=11)$} & \multicolumn{2}{|c|}{ Late-window $(n=13)$} & \multirow[b]{2}{*}{ P-value } \\
\hline & ASPECTS & ICC (IQR) & ASPECTS & ICC (IQR) & \\
\hline DWI-ASPECTS & $4.8 \pm 2.7$ & & $4.8 \pm 2.4$ & & \\
\hline $\begin{array}{l}\text { Pooled NCCT- } \\
\text { ASPECTS }\end{array}$ & $8.3 \pm 1.7$ & $0.65(0.32-0.88)$ & $7.1 \pm 2.2$ & $0.64(0.34-0.86)$ & 0.01 \\
\hline $\begin{array}{l}\text { Pooled VIM- } \\
\text { ASPECTS }\end{array}$ & $6.9 \pm 2.5$ & $0.57(0.21-0.84)$ & $6.0 \pm 2.5$ & $0.61(0.30-0.84)$ & 0.18 \\
\hline $\begin{array}{l}\text { P-value } \\
\text { (vertical) }\end{array}$ & $<0.001$ & & $<0.001$ & & \\
\hline \multicolumn{6}{|c|}{ Individual Reader ASPECTS Comparisons } \\
\hline Early-window & NCCT-ASPECTS & VIM-ASPECTS & P-value (1) & P-value (2) & P-value (3) \\
\hline Reader 1 & $7.9 \pm 1.7$ & $6.2 \pm 2.5$ & 0.004 & 0.008 & 0.002 \\
\hline Reader 2 & $8.2 \pm 1.8$ & $7.3 \pm 2.0$ & 0.33 & 0.03 & 0.02 \\
\hline Reader 3 & $8.7 \pm 1.6$ & $7.2 \pm 2.9$ & 0.49 & 0.27 & 0.02 \\
\hline \multicolumn{6}{|l|}{ Late-window } \\
\hline Reader 1 & $6.8 \pm 2.5$ & $5.8 \pm 2.3$ & 0.02 & 0.008 & 0.01 \\
\hline Reader 2 & $7.2 \pm 2.0$ & $5.9 \pm 2.8$ & 0.31 & 0.004 & 0.04 \\
\hline Reader 3 & $7.4 \pm 2.2$ & $6.5 \pm 2.8$ & 0.63 & 0.27 & 0.07 \\
\hline
\end{tabular}

Abbreviations: VIM; virtual ischemia maps, NCCT; non-contrast CT, DWI; diffusion-weighted imaging, ICC; intraclass correlation coefficient. 

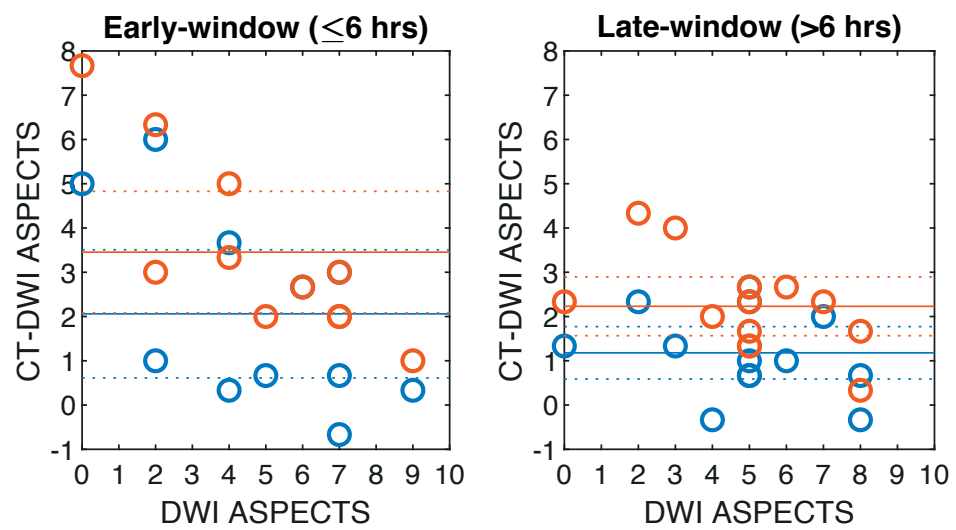

○ VIM O NCCT

Figure 6.7 | Bland-Altman analyses plotting the difference between each CT- and DWI-ASPECTS versus corresponding DWI-ASPECTS are presented for both early- and late-window patients. Solid lines indicate the mean bias value for each measurement, and hashed lines indicate the 95\% agreement interval limits. Values closer to 0.0 indicate greater agreement with the DWI-ASPECTS.

\section{Discussion}

In this study, we found that DECT VIM more accurately estimated core infarct volumes than NCCT or CTP compared to reference standard DWI assessment in AIS-LVO patients. CT remains the mainstay in the imaging triage of suspected AIS-LVO patients for ET, though there have been limited improvements in the non-contrast assessment of core infarction. The increased accuracy of VIM core infarct delineation over NCCT is of particular clinical relevance given established core volume thresholds in determining ET candidacy[14,99,107,108]. While our findings also suggest that VIM more accurately estimated core infarction than CTP, commonly applied CBF<30\% segmentation thresholds underestimate the core volume relative to a less restrictive $\mathrm{CBF}<38 \%$ threshold to reduce false exclusion of patients from ET[109]. Consequently, we suggest that VIM may be an adjunct to, but not replace CTP core estimates during AIS-LVO patient triage.

Our analysis of multi-reader ASPECTS found that VIM was non-inferior to NCCT, with a trend toward improved qualitative conspicuity of core infarction relative to DWIASPECTS (mean bias 1.58 vs 2.79). Interrater agreement was moderate for both VIM and NCCT and concordant with previously reported interrater variability in ASPECTS determination[110]. The lack of significant differences in ASPECTS determination and interrater variability in our study suggests that a larger sample size is necessary to determine if the trend toward superior VIM-ASPECTS accuracy is significant beyond known limitations of heterogeneity in ASPECTS interpretation. Nonetheless, our results may remain clinically relevant in risk stratification of AIS-LVO patients, as larger visible ischemic cores assessed by ASPECTS portend a lower likelihood of benefit from endovascular therapy[111]. 
Subgroup analyses of AIS-LVO patients presenting in the early- ( $\leq 6$ hours) versus latewindow ( $>6$ hours) demonstrated superior normalized core infarct estimates using VIM over NCCT in both early- and late-windows. Bland-Altman analyses indicated significantly greater accuracy of VIM over NCCT core estimation in the early-window (mean bias 0.60 vs. 0.20), but only a trend toward greater accuracy in the late-window (mean bias 0.75 vs. 0.45). As perfusion imaging is not necessary in early-window AISLVO patient triage, the superiority of VIM over NCCT in core infarct determination may better direct early-window treatment decisions[10,111,112]. The non-significant trend toward greater accuracy of VIM over NCCT in the late-window may be attributed to timedependent maturation of edema within the infarct, which is concordant with the smaller difference in normalized CT core volumes observed between time-windows[113,114].

Relative to NCCT, VIM reconstructions qualitatively demonstrate a more uniform attenuation between gray and white matter, with areas of hypoattenuation in acute infarction appearing more starkly contrasted. We hypothesize that the VIM multimaterial separation algorithm using hemorrhage, water, and iodine definitions generates this homogenized appearance by assuming that individual voxel attenuation can be attributed to the relative contribution of only the base-pair materials[87,115]. In these non-contrast examinations, iodine content is zero, therefore the VIM acts as a two-material separation algorithm and subsequently highlights altered water content within the homogenized parenchyma, particularly in areas of early developing ionic and cytotoxic edema within the core infarct. This proposed mechanism is compatible with our observation that VIM is relatively superior to NCCT in the early-window, during which time ischemia results in more subtle predominantly ionic and cytotoxic edema[116]. Conversely, in the late-window, vasogenic edema progresses within the maturing ischemic lesion, which may explain the overall greater agreement between VIM and NCCT in predicting the DWI core[116,117].

Relative to previously described DECT-based edema reconstructions, which performed custom material decompositions using material basis pairs of either air and water, or calcium and fat, VIM reconstructions are a repurposing of a readily available brain hemorrhage reconstruction module using manufacturer default settings[89,91]. Our method corroborates prior findings that DECT techniques may improve acute infarct conspicuity, while demonstrating that even a simple, default reconstruction technique is capable of estimating core infarction in a carefully selected AIS-LVO population with accuracy comparable to that of perfusion-based estimates and approaching that of DWI, which remain the current reference standards in practice and were not used to estimate true infarct volume in previous studies. 
Our study has several limitations. First, we selected only patients in whom there is a matched perfusion profile or who undergo expeditious and complete or nearcomplete revascularization. Therefore, we assume that core infarct size should not grow or only minimally progress from admission CT to MRI, but we are unable to assess if progression did in fact occur. Second, our study may be biased by heterogeneous patient treatment and sites of occlusion. Third, ASPECTS assessment is limited by established inter-reader variability, small sample size, and heterogeneity in experience among readers, necessitating further testing to determine if VIM may provide a more reliable basis for ASPECTS determination[110,118]. Fourth, limited utilization of CT for the triage of AIS-LVO patients at our institution and reduced penetration of DECT protocols due to patient motion or body habitus may introduce selection bias. Finally, our study uses only a single-vendor DECT scanner and VIM algorithm, which reduces variability in image quality, but limits generalizability. However, VIM may theoretically be reconstructed without regard to vendor, as all DECT scanners are capable of material decomposition and most vendors include comparable default brain hemorrhage decomposition modules.

We conclude that non-contrast DECT VIM may more accurately estimate core infarction than conventional NCCT in AIS-LVO patients, especially in the early time window $(\leq 6$ hours). Currently, AIS-LVO triage is critically reliant upon accurate neuroimaging to appropriately determine treatment candidacy, with CT remaining the most widely available and utilized imaging modality[10,119]. We expect that neurointerventionalists with practices reliant on CT imaging will benefit from the improved infarct detection profile offered by the VIM technique, especially for early-window AIS-LVO patients in whom CTP may not be routinely performed or available. Larger prospective validation of core infarct estimation by VIM is warranted. 



\title{
CHAPTER 7
}

\section{VIRTUAL MONOENERGETIC CT PERFUSION}

\author{
Based on
}

Fvan Ommen, E Bennink, JW Dankbaar, F Kauw and HWAM de Jong (2020). Improving the quality of cerebral perfusion maps with monoenergetic dual-energy CT

reconstructions. Journal of Computer Assisted Tomography, in press. 
Part II | Dual-energy CT in stroke imaging

\section{Abstract \\ Objectives}

We compared 40 - 70 keV virtual monoenergetic to conventional CT perfusion reconstructions with respect to quality of perfusion maps.

\section{Methods}

Conventional CTP images were acquired at $80 \mathrm{kVp}$ in 25 patients and $40-70 \mathrm{keV}$ images were acquired with a dual-layer CT at $120 \mathrm{kVp}$ in 25 patients. First, timeattenuation-curve contrast-to-noise ratio (CNR) was assessed. Second, the perfusion maps of both groups were qualitatively analyzed by observers. Last, the monoenergetic reconstruction with the highest quality was compared to the clinical standard $80 \mathrm{kVp}$ CTP acquisitions.

\section{Results}

CNR was significantly better for 40 - $60 \mathrm{keV}$ as compared to $70 \mathrm{keV}$ and conventional images $(p<0.001)$. Visually, the difference between the cerebral blood volume maps among reconstructions was minimal. The $50 \mathrm{keV}$ perfusion maps had the highest quality compared to the other monoenergetic and conventional maps $(p<0.002)$.

\section{Conclusions}

The quality of $50 \mathrm{keV}$ CT perfusion images is superior to the quality of conventional 80 and $120 \mathrm{kVp}$ images. 


\section{Introduction}

Patients admitted to the emergency department with suspected acute ischemic stroke are often evaluated with $\mathrm{CT}$ imaging. Acute stroke imaging protocols typically include non-contrast CT, CT perfusion (CTP) and CT angiography.

A CTP scan is a dynamic acquisition of the brain parenchyma, to monitor the washin and wash-out of iodinated contrast agent[120]. From this series of scans, a set of perfusion parameters can be calculated, including cerebral blood flow (CBF), cerebral blood volume (CBV) and mean transit time (MTT). These maps can be used to visualize the perfusion of brain tissue, and assist in differentiating between healthy, salvageable (infarct penumbra) and lost (infarct core) tissue[121-123], which are important parameters in therapy guidance[108]. For generating high quality CTP parameter maps, optimal visualization of the iodine contrast agent is key. CTP is therefore commonly acquired at $80 \mathrm{kVp}$, which is often the lowest available tube voltage and closest to the k-edge of iodine (33.2 keV).

Dual-energy CT imaging is a technique in which two datasets are acquired at two different (average) energies. Dual-energy CT acquisition can be used to reconstruct conventional CT images, but also various images that exploit the dual-energy information such as virtual non-contrast[70], iodine-only[124], effective atomic number[125] and virtual monoenergetic[30,43] CT images. Dual-energy CT data can be acquired by using dual X-ray sources[126], kVp switching[127], or a dual-layer CT scanner. The dual-layer $\mathrm{CT}$ utilizes a single $\mathrm{X}$-ray tube and a dual-layer detector, implementing separation of the energy spectrum at the detector level. By default, dual-energy data is acquired during all acquisitions, but proper dual-energy separation is only possible at higher tube voltages (i.e. 120 and 140 kVp). Thus, to exploit the dual-energy information with CTP imaging the acquisition protocol has to be changed from $80 \mathrm{kVp}$ to $120 \mathrm{kVp}$. Although this may result in reduced contrast in the conventional $120 \mathrm{kVp}$ CTP reconstructions, it enables generation of virtual monoenergetic CT images in the range from 40 to $200 \mathrm{keV}$. The low keV (40 - $50 \mathrm{keV}$ ) images include data much closer to the iodine k-edge at 33.2 keV, potentially increasing the iodine contrast of the CTP acquisitions. The generation of the low keV virtual monoenergetic CT images, however, comes at the cost of increased noise levels compared to a conventional CTP acquisition. It is hypothesized that the gain in contrast outweighs these increased noise levels, but supporting evidence is lacking. Therefore, the purpose of this study was to compare the image quality of CTP acquisitions and the quality of perfusion parameter maps of conventional 80 and 120 kVp scans and low keV virtual monoenergetic reconstructions. 


\section{Part II | Dual-energy CT in stroke imaging}

\section{Materials and Methods}

We retrospectively identified 25 consecutive patients imaged with the $80 \mathrm{kVp}$ CTP protocol and 25 patients who were scanned on the dual-layer CT at $120 \mathrm{kVp}$. Patients suspected for stroke imaged on the dual-layer $\mathrm{CT}$, at least 18 years old and a complete CTP acquisition were included. Exclusion criteria were insufficient quality of the CTP acquisition to perform adequate measurements (i.e. severe motion or artifacts). The local medical ethics committee approved the study, and the need for informed consent was waived.

\section{Patient data}

\section{CTP $80 \mathrm{kVP}$}

For this evaluation, 25 consecutive patients scanned at $80 \mathrm{kVp}$ were selected. The CTP acquisition consisted of 22 frames, with an axial coverage of 8 centimeters using Jog Mode, giving an acquisition interval of approximately 3.5 seconds. The dynamic series were acquired at $100 \mathrm{mAs}$ per rotation, resulting in a CTDI of 6 mGy. This examination was acquired without any initial time delay, with a rotation time of 0.33 seconds, $5 \mathrm{~mm}$ reconstructed slice thickness, 512×512 image matrix, collimation width of $64 \times 0.625$ $\mathrm{mm}$ and a field-of-view of $250 \mathrm{~mm}$. Conventional CT images were reconstructed using iDose 4 level 5, and a "UA" filter kernel. For each CTP series, a $50 \mathrm{ml}$ bolus of iodine (Ultravist-300 mg/ml, Bayer) was administered, followed by $70 \mathrm{ml}$ saline solution at an injection rate of $6 \mathrm{ml} / \mathrm{s}$.

\section{CTP $120 \mathrm{kVp}$}

At our hospital, with the introduction of the dual-layer CT scanner, all CT protocols were changed to dual-energy CT acquisition (120 kVp acquisitions). For all new protocols the acquisition settings were adjusted to match the dose of the old protocol[128]. In addition, 25 patients scanned at $120 \mathrm{kVp}$ on the dual-layer CT were selected. The 120 $\mathrm{kVp}$ acquisition protocol was copied from the $80 \mathrm{kVp}$, but the protocol was designed to match the CTDI of 6 mGy of the $80 \mathrm{kVp}$ CTP acquisition, which resulted in an exposure of $35 \mathrm{mAs}$ per rotation. All other acquisition parameters were kept constant compared to the $80 \mathrm{kVp}$ acquisition. Similar to the $80 \mathrm{kVp}$ acquisition, images were reconstructed using iDose ${ }^{4}$ level 5, and a "UA" filter kernel.

From the $120 \mathrm{kVp}$ acquisition, conventional CT and dual-energy datasets were available. The dual-energy datasets were used to create monoenergetic CTP reconstructions using the spectral CT viewer (ISP9; IntelliSpace Portal 9, Philips Healthcare, Best, the Netherlands). Virtual monoenergetic CTP datasets were reconstructed at 40, 50, 60, and $70 \mathrm{keV}$. We choose to use the 40 - $70 \mathrm{keV}$ reconstructions, because we only expect an increase in contrast for the low keV images as the iodine k-edge is $33.2 \mathrm{keV}$. 


\section{Perfusion analysis}

CTP analysis is a so-called inverse problem. A known arterial input function (AIF) and tissue attenuation curves (TACS) are used to derive the impulse response function (IRF) using the following (de)convolution:

$$
T A C(t)=I R F(t) * A I F(t) .
$$

Non-linear regression deconvolution is a non-linear model-based approach to solve this inverse problem. In this approach, an initial guess of the impulse response function is convolved with the known AIF:

$$
T A C_{e s t}(t)=I R F_{\text {est }}(t) * A I F(t)+\varepsilon(t) .
$$

Next, non-linear regression is used to iteratively update the impulse response function estimate to minimize the error between the estimated and measured time attenuation curve. From the estimated impulse response function, the cerebral blood flow (CBF), cerebral blood volume (CBV) and mean transit time (MTT) can be calculated[17].

\section{Pre-processing}

Prior to the perfusion analysis, scans were corrected for motion using a 3D rigid registration on the skull, using the registration software package Elastix[129]. Following registration, the datasets were filtered using a bilateral filter[17]. The required strength of the bilateral filter was determined by the noise in the guide image (size of range kernel) and the initial signal-to-noise ratio (SNR) of the scan (size of spatial kernel) before filtering. The SNR was defined as the amplitude of the AIF divided by the noise (SD) before the bolus arrival (Figure 7.1):

$$
S N R=\frac{A I F_{\text {amplitude }}}{S D_{\text {noise }}}
$$

The noise is calculated by taking the frames before the bolus arrival, and calculating the average noise of all pixels. Each dataset was then filtered until in the filtered image a new SNR of at least 400 was found, this value was chosen based on visual inspection. 


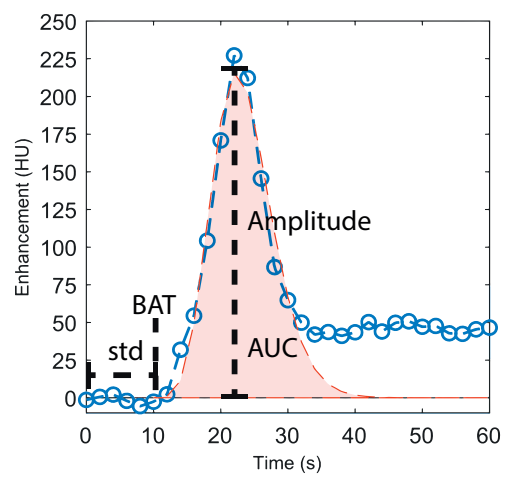

Figure 7.1 | Simulated AIF to illustrate the used variables in the CNR and SNR calculations. In which, AUC stands for area under the curve of the AIF, SD for noise before the bolus arrival, BAT the bolus arrival time and Amplitude for the amplitude of the AIF.

\section{Image analysis}

Quantitative analysis

To test the initial image quality of the unfiltered $\mathrm{CT}$ perfusion reconstructions, a contrast-to-noise ratio (CNR) was devised. The AIF and venous output function were selected as the TACs with the largest enhancements, from which a partial volume effect corrected AIF was calculated. The noise of the frames before the bolus arrival resembles the noise fraction of the CNR, and the area under the curve (AUC) of the corrected AIF resembles contrast (Figure 7.1). Based on the assumption that in all kVp and monoenergetic reconstructions from the same patient the width of the bolus was equal, we calculated a measure for the CNR as:

$$
C N R=\frac{A I F_{A U C}}{S D_{\text {noise }}} .
$$

In addition, the perfusion values of the different reconstructions were compared. For the analysis of the perfusion maps, two identical circular regions of interest (ROIs) were drawn in each hemisphere, one in the gray matter (GM) and one in the white matter (WM). The ROIs were the same for the monoenergetic and $120 \mathrm{kVp}$ reconstructions, for $80 \mathrm{kVp}$ a separate set of ROIs was used. These ROIs were used to calculate the average perfusion parameters (CBV, CBF and MTT) for each reconstruction.

\section{Qualitative analysis}

In addition, a qualitative analysis was performed on the quality of the perfusion parameter maps with an observer study. First, an experienced neuroradiologist (JWD) scored overall quality of the different monoenergetic parameter map reconstructions and $120 \mathrm{kVp}$. Overall quality includes: the ability to recognize anatomy, the presence of noise and artifacts in the perfusion maps. The observer was blinded to clinical information and source reconstructions. The observer was able to control window width and window level of the image display of the different perfusion maps. 
From every patient, the center slice of the CBF, CBV and MTT map of the different reconstruction were randomly presented as a pairwise comparison to the observer. This resulted in 10 different combinations for every perfusion parameter map, namely; $120 \mathrm{kVp}-40 \mathrm{keV}, 120 \mathrm{kVp}-50 \mathrm{keV}, 120 \mathrm{kVp}-60 \mathrm{keV}, 120 \mathrm{kVp}-70 \mathrm{keV}, 40 \mathrm{keV}-50 \mathrm{keV}$, $40 \mathrm{keV}-60 \mathrm{keV}, 40 \mathrm{keV}-70 \mathrm{keV}, 50 \mathrm{keV}-60 \mathrm{keV}, 50 \mathrm{keV}-70 \mathrm{keV}$ and $60 \mathrm{keV}-70 \mathrm{keV}$. The observer scored which of the two parameter maps was superior in quality. The observer scored 750 comparisons in total. This initial comparison was to identify the reconstruction with the highest quality maps. This reconstruction was subsequently compared to the current clinical standard of $80 \mathrm{kVP}$ CTP acquisitions.

From the first intra-patient observer study, the $50 \mathrm{keV}$ monoenergetic reconstruction was selected as the one with the highest quality. The $50 \mathrm{keV}$ monoenergetic reconstructions were then compared to the conventional $80 \mathrm{kVp}$ acquisitions (the current clinical standard) in an inter-patient observer study. From both reconstructions, the center slice and the two adjacent slices of the CBF, CBV and MTT maps were collected. This resulted in 75 slices per perfusion map per patient group. Two observers (an experienced radiologist (JWD) and a radiology trainee (FK)) identified which CBF, CBV or MTT map had the highest quality in a pairwise comparison between a patient from the $80 \mathrm{kVp}$ and a patient from the $50 \mathrm{keV}$ monoenergetic reconstruction group. Each observer received a different random set of image pairs. As a result, each observer received 225 comparisons in total.

\section{Statistical analysis}

To evaluate difference between the $80 \mathrm{kVp}$ and $120 \mathrm{kVp}$ patient group a Mann-Whitney $U$ test was used for the continuous variables (age) and a Chi-squared test for discrete variables (sex, number of infarcts etc.). Differences in AUC, noise, CNR and perfusion values of the different monoenergetic and $120 \mathrm{kVp}$ reconstructions were tested for significance using a repeated measures analysis of variance (ANOVA). Differences in AUC, noise, CNR and perfusion values between $80 \mathrm{kVp}$ and other reconstructions were tested for significance using an unpaired sample t-test. For the qualitative study, the qualitative analysis between the different reconstructions was tested for significance using a Chi-squared test. The significance level was set to 0.05. Analyses were performed in SPSS version 25.0 (IBM, Armonk, NY, USA).

\section{Results}

\section{Patient data}

A total of 50 patients with suspected stroke were included in this study. The median age of the $80 \mathrm{kVp}$ group was 66 years, and 17 patients were male. For the $120 \mathrm{kVp}$, the median age was 70 and of 25 patients 15 were male. Not all patients were diagnosed with an infarct or TIA in both groups. There were no significant differences between the two groups (Table 7.1). 
Part II | Dual-energy CT in stroke imaging

Table 7.1 | Patient characteristics and diagnosis of patients scanned at $80 \mathrm{kVp}$ and $120 \mathrm{kVp}$.

\begin{tabular}{llll}
\hline Characteristics & $80 \mathrm{kVp}(\mathrm{n}=25)$ & $120 \mathrm{kVp}(\mathrm{n}=25)$ & P-value \\
\hline Sex, male:female & $17: 8$ & $15: 10$ & 0.556 \\
Age (years), median (IQR) & $66(33-94)$ & $70(21-87)$ & 0.356 \\
Diagnosis & & & \\
$\quad$ Infarct, $\mathrm{n}(\%)$ & $12(48)$ & $9(36)$ & 0.390 \\
TIA, $\mathrm{n}(\%)$ & $2(8)$ & $2(8)$ & 1.000 \\
Other, $\mathrm{n}(\%)$ & $11(44)$ & $14(56)$ & 0.396 \\
\hline
\end{tabular}

Abbreviation: IQR; interquartile range.

\section{Quantitative analysis}

In Figure 7.2, the mean AUC, noise and CNR of the different reconstructions before filtering are shown. In general, the AUC and CNR of the 40, 50 and $60 \mathrm{keV}$ monoenergetic reconstructions were higher than those of the $70 \mathrm{keV}$ and conventional reconstructions. The AUC and the noise (Figure 7.2) are shown to illustrate the differences between different reconstructions. The CNR (Figure 7.2), showed no significant difference between the 80 and $120 \mathrm{kVp}$ reconstructions ( $p=0.265$ ). The CNR of the 40, 50 and 60 $\mathrm{keV}$ monoenergetic reconstructions was significantly higher compared to the $80 \mathrm{kVp}$ reconstructions ( $p<0.005)$. The CNR of the $80 \mathrm{kVp}$ reconstruction compared to the $70 \mathrm{keV}$ was not significantly different ( $p=0.765)$. In addition, the CNR of the different monoenergetic and $120 \mathrm{kVp}$ reconstructions were significantly different from each other $(p<0.001)$.
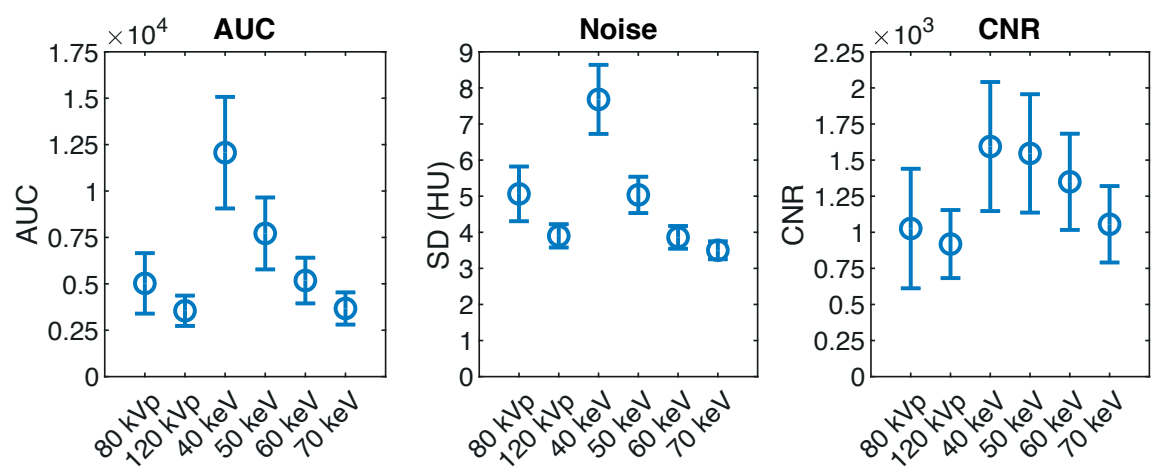

Figure 7.2 | The area under the curve (AUC) of the AIF, the noise (SD) and contrast-to-noise ratio (CNR) of the different CTP reconstructions before filtering.

Figure 7.3, illustrates the difference between the different reconstructions. The virtual monoenergetic reconstructions are derivatives of the $120 \mathrm{kVp}$ source data. Contrast is higher on the low keV monoenergetic reconstructions, but also noise is higher. 


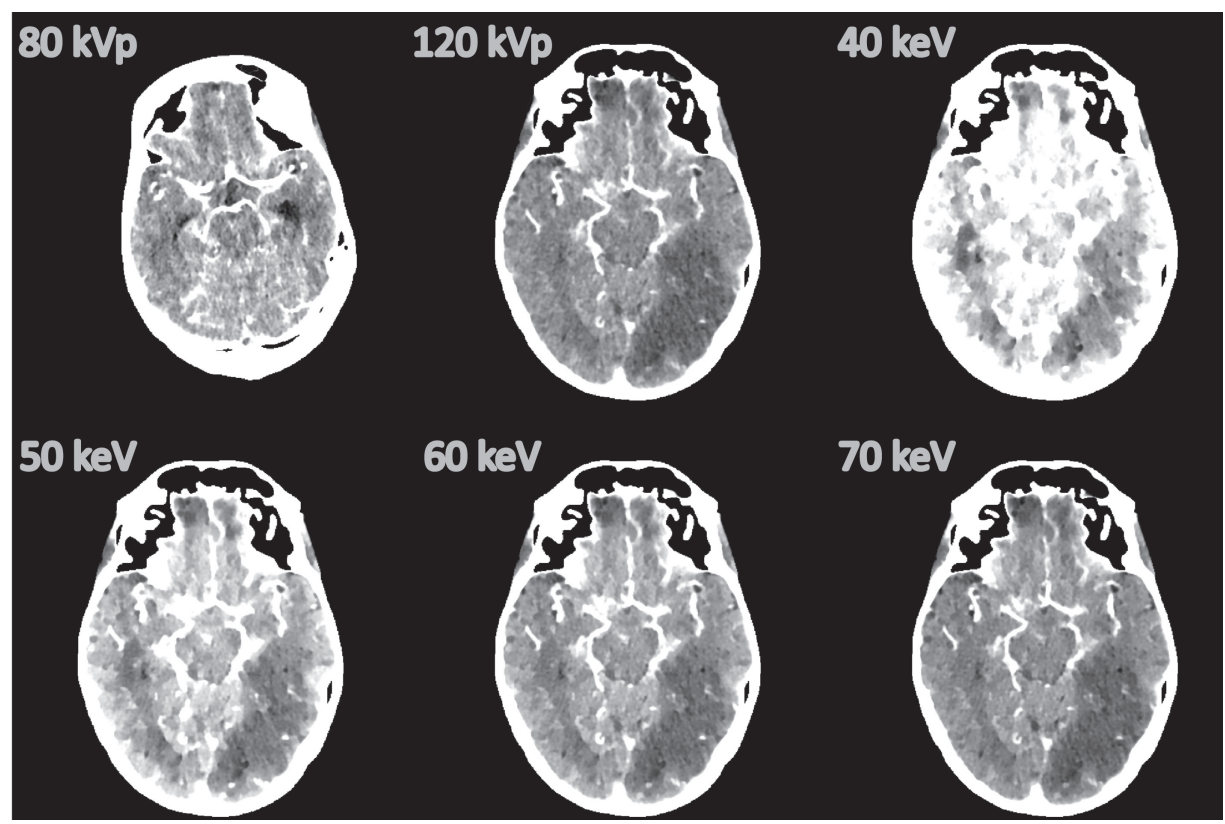

Figure 7.3 | Characteristic images of the different reconstructions of the patient group scanned at $80 \mathrm{kVp}$, and of the patient group at $120 \mathrm{kVp}$, including the virtual monoenergetic reconstructions. The window level and width is set to $40 / 80 \mathrm{HU}$, window settings were kept equal for comparability.

In Figure 7.4, the CBF, CBV and MTT values of the gray matter are shown. There was a significant effect on CBF, CBV and MTT values for monoenergetic reconstructions compared to $120 \mathrm{kVp}(\mathrm{p}<0.022) .80 \mathrm{kVp}$ compared to $120 \mathrm{kVp}$ did not show a significant difference for CBF ( $p=0.470)$, the CBF of monoenergetic reconstructions were significantly lower compared to $80 \mathrm{kVp}$ ( $p<0.020)$, except for $70 \mathrm{keV}(p=0.138)$. The CBV of $80 \mathrm{kVp}$ was significant higher compared to other reconstructions $(p<0.04)$. The MTT values of 80 $k V p$ was not significantly different compared to the other reconstructions ( $p>0.095)$.
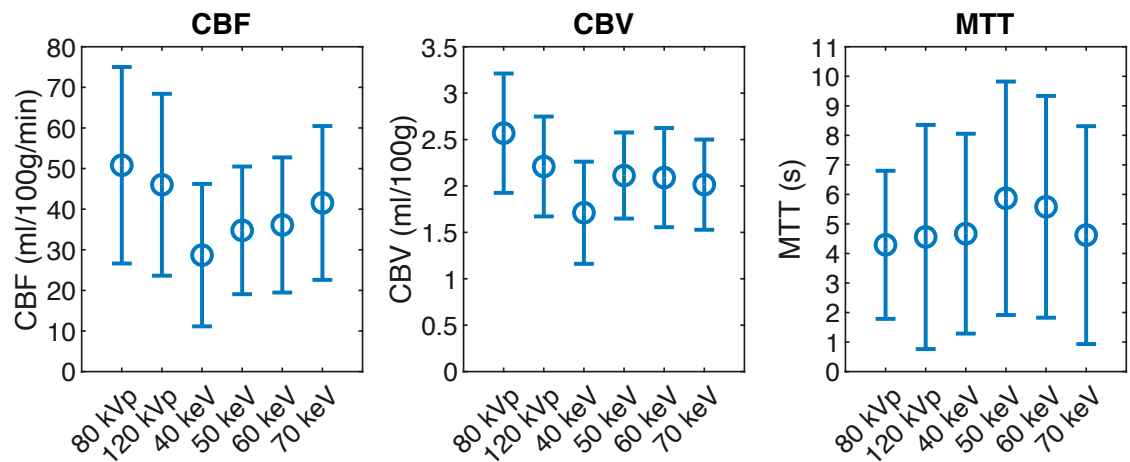

Figure 7.4 | Mean CBF, CBV and MTT values of the different CTP reconstructions of the gray matter, measured in two ROIs of $13 \mathrm{~mm}^{2}$ in both hemispheres. The $80 \mathrm{kVp}$ patient group is different from the $120 \mathrm{kVp}$ patient group. 
Figure 7.5, shows the CBF, CBV and MTT values for the white matter. The effect of monoenergetic reconstructions on CBV and MTT compared to $120 \mathrm{kVp}$ was significant $(p<0.013)$, whereas the CBF values were not significantly affected $(p=0.072)$. The CBF, CBV and MTT of $80 \mathrm{kVp}$ compared to the other reconstructions were not significantly different ( $p>0.085)$.
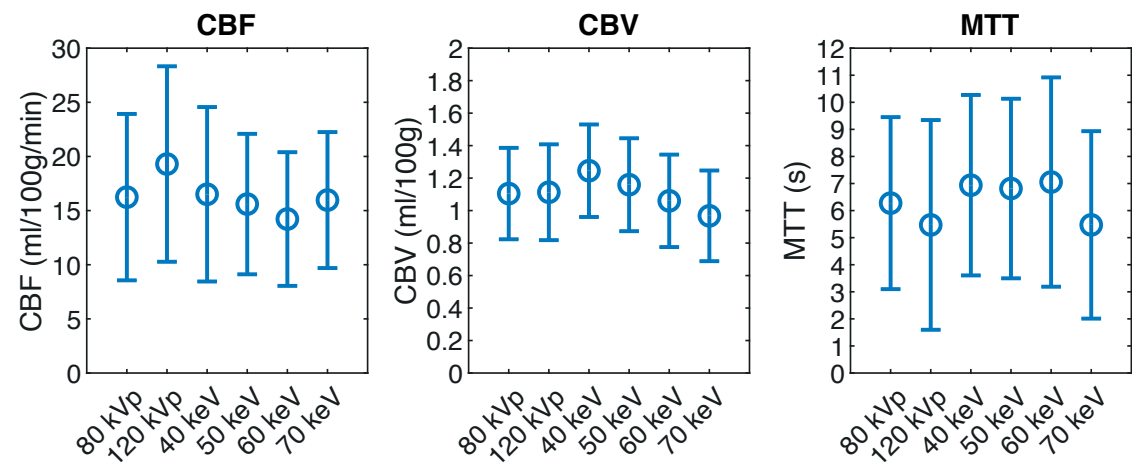

Figure 7.5 | Mean CBF, CBV and MTT values of the different CTP reconstructions of the white matter, measured in two ROIs of $13 \mathrm{~mm}^{2}$ in both hemispheres. The $80 \mathrm{kVp}$ patient group is different from the $120 \mathrm{kVp}$ patient group.

\section{Qualitative analysis}

$120 \mathrm{kVp}$ and monoenergetic reconstructions

Figure 7.6 shows the result of the visual assessment of the different perfusion parameter maps. Figure 7.6A shows the result for the CBF maps. The observer deemed the lower monoenergetic reconstructions (i.e. 40 and $50 \mathrm{keV}$ monoenergetic) as having a higher quality compared to the higher monoenergetic reconstructions (i.e. 60 and $70 \mathrm{keV}$ ), even though the differences are small. The difference in quality between the monoenergetic reconstructions and the $120 \mathrm{kVp}$ source data is larger. Both the 40 and $50 \mathrm{keV}$ reconstruction was found to be better than $120 \mathrm{kVp}$ reconstruction, but the $40 \mathrm{keV}$ was found to be slightly superior compared to the $50 \mathrm{keV}$ reconstructions. The difference between $120 \mathrm{kVp}-40 \mathrm{keV}, 40 \mathrm{keV}-50 \mathrm{keV}, 40 \mathrm{keV}-60 \mathrm{keV}$ and $60 \mathrm{keV}$ - 70keV were not significantly different ( $p>0.150$ ); all other comparison showed a significant difference in selection $(p<0.050)$.

Figure 7.6B shows the results for the CBV maps. The $120 \mathrm{kVp}$ performed better for the 40 and $50 \mathrm{keV}$, whereas the 60 and $70 \mathrm{keV}$ outperformed $120 \mathrm{kVp}$. In addition, the low keV monoenergetic reconstructions were selected less often as superior compared to the higher monoenergetic reconstructions. The $120 \mathrm{kVp}-50 \mathrm{keV}$ and $120 \mathrm{kVp}-60$ keV comparisons did not show a significant difference ( $p>0.390)$. All other comparison showed significant differences $(p<0.050)$. 
Figure 7.6C shows the results for the MTT map. The lower monoenergetic reconstructions were of superior quality compared to the higher monoenergetic reconstructions and the $120 \mathrm{kVp}$ source data. The comparison between $120 \mathrm{kVp}-70$ $\mathrm{keV}$ and $40 \mathrm{keV}$ - $50 \mathrm{keV}$ did not show a significant difference ( $p>0.150)$, whereas all other comparisons were significant different from each other $(p<0.050)$.

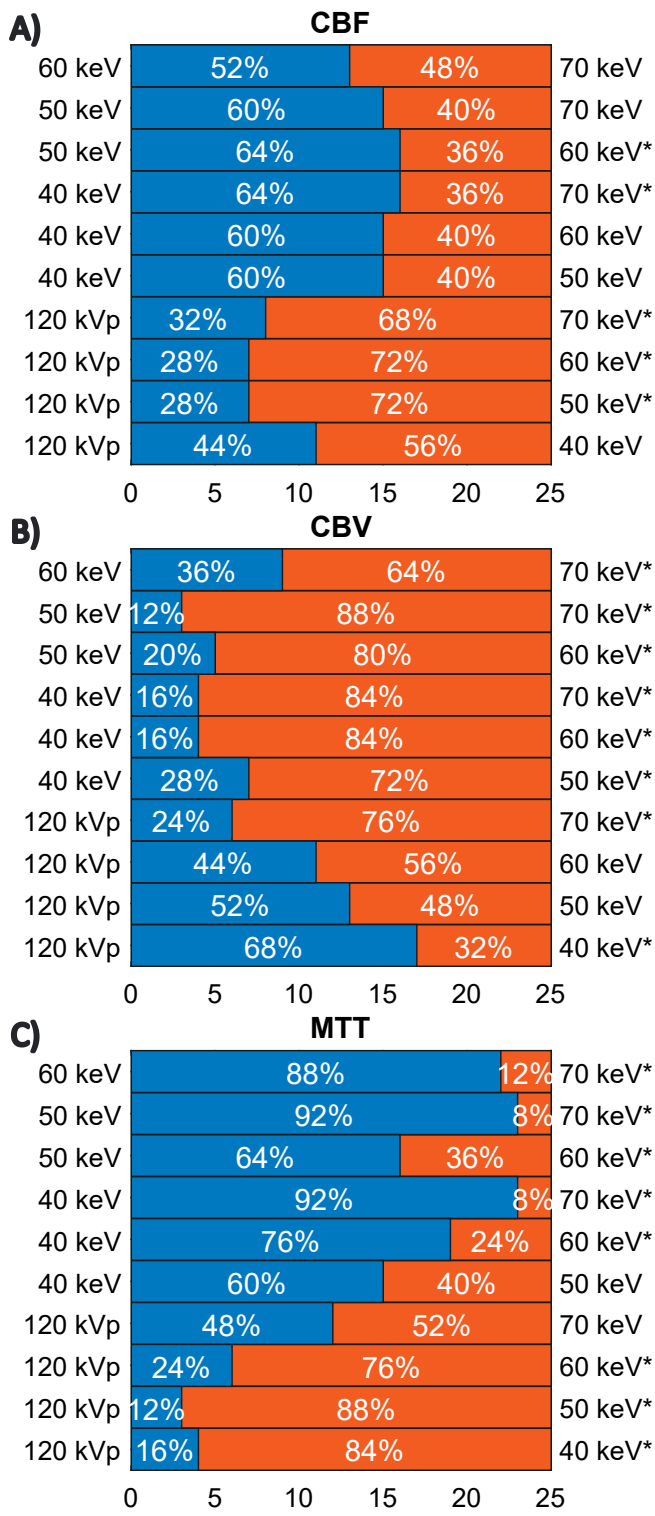

Figure 7.6 | Comparison of overall quality amongst various monoenergetic and $120 \mathrm{kVp}$ reconstructions for the A) CBF, B) CBV and c) MTT maps. On the x-axis the number of comparisons is given. If the difference between the reconstructions is significant, it is marked with an asterisk. 
In Figure 7.7, an example of the perfusion parameter maps from the different monoenergetic reconstructions and 120 kVp source data are shown.

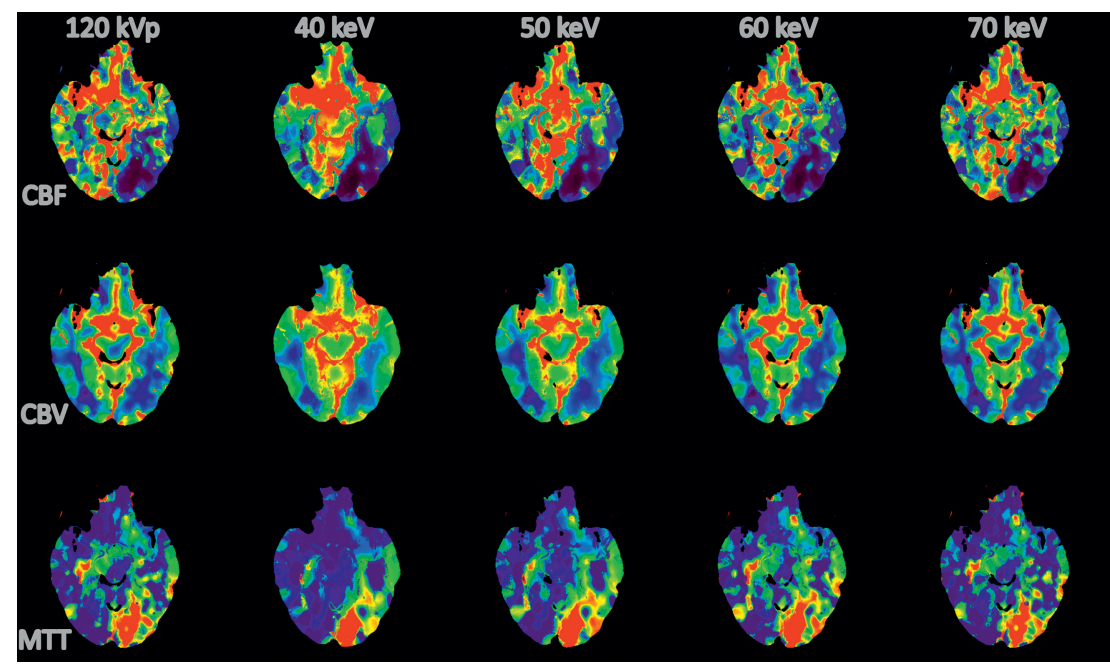

Figure 7.7 | Characteristic CBF, CBV and MTT maps from the same patient for the different monoenergetic reconstructions and $120 \mathrm{kVp}$ source data. The quality of the CBF and MTT improves significantly with 40 and $50 \mathrm{keV}$ reconstructions compared to the other reconstructions. The CBV map did not differ too much between the different reconstructions.

The results in Figure 7.6 showed that the 40 or $50 \mathrm{keV}$ monoenergetic reconstructions were identified as being superior. For the CBF and MTT the difference between 40 and $50 \mathrm{keV}$ was not significant, but for the CBV maps $50 \mathrm{keV}$ was significantly superior. Therefore, $50 \mathrm{keV}$ was chosen as the superior monoenergetic reconstruction and compared to the $80 \mathrm{kVp}$ clinical standard.

\section{$80 \mathrm{kVp}$ and $50 \mathrm{keV}$}

In Table 7.2, the visual assessment of $80 \mathrm{kVp}$ compared to $50 \mathrm{keV}$ is shown. In general, the perfusion parameter maps from the $50 \mathrm{keV}$ monoenergetic reconstructions outperform the perfusion parameter maps from the $80 \mathrm{kVp}$ reconstructions. Both observers found that, for all perfusion parameter maps, the $50 \mathrm{keV}$ reconstructed CTP images were significantly superior compared to the $80 \mathrm{kVp}$ reconstructed $(p<0.002)$.

Table 7.2 The selection preference for $50 \mathrm{keV}$ over the $80 \mathrm{kVp}$ reconstruction of observer 1 and observer 2 for all three parameter maps (CBF, CBV and MTT). The significant differences are marked with asterisks. The $p$-value is given between brackets.

\begin{tabular}{lll}
\hline & Observer 1 & Observer 2 \\
\hline CBF & $71 \%(p<0.001)^{\star}$ & $69 \%(p<0.001)^{*}$ \\
CBV & $81 \%(p<0.001)^{*}$ & $77 \%(p<0.001)^{*}$ \\
MTT & $63 \%(p=0.002)^{*}$ & $71 \%(p<0.001)^{*}$ \\
\hline
\end{tabular}


To illustrate the difference between the different perfusion parameter maps of the $80 \mathrm{kVp}$ reconstruction and $50 \mathrm{keV}$, characteristic perfusion parameter maps of both reconstructions are shown in Figure 7.8.

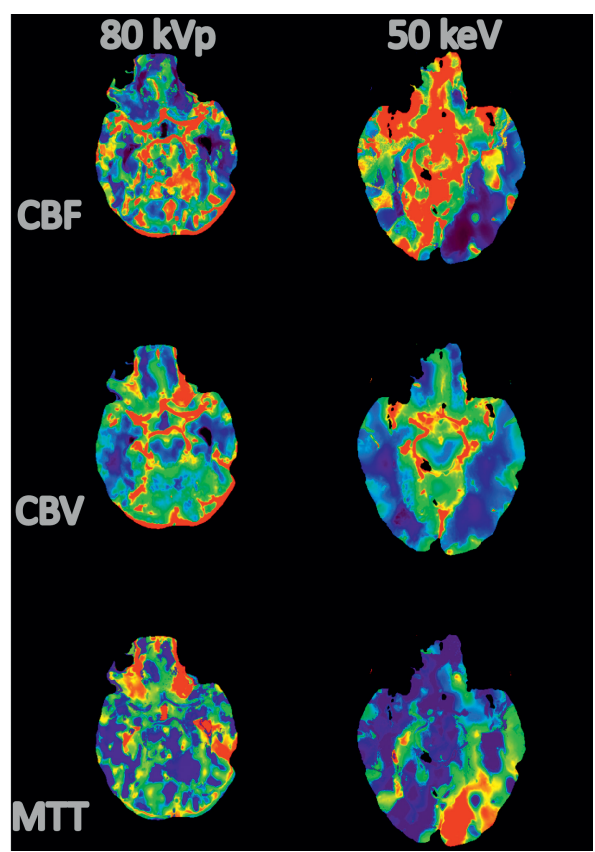

Figure 7.8 | Characteristic CBF, CBV and MTT maps from the $80 \mathrm{kVp}$ and $50 \mathrm{keV}$ reconstructions. The quality improves significantly with the $50 \mathrm{keV}$ reconstruction compared to the $80 \mathrm{kVp}$ reconstruction.

\section{Discussion}

In this study, the value of low keV virtual monoenergetic CT perfusion from spectral CT scans was assessed by evaluating the image quality of CTP acquisitions and the quality of perfusion parameter maps of conventional 80 and $120 \mathrm{kVp}$ scans and low keV virtual monoenergetic reconstructions. In recent years, many strategies have been evaluated to improve the quality of CTP scans including adjustment of the amount of contrast agent[130], the acquisition interval[131] and the use of iterative reconstructions[132]. In cerebral non-contrast CT images the use of monoenergetic images from dual energy or spectral CT has shown improved gray-white matter separation and increased CNR compared to conventional singe energy CT[50], but also increased sensitivity for detection of infarct in non-contrast CT images[23,94]. However, to our knowledge there has not yet been a study evaluating the use of monoenergetic images in CTP to find the optimal balance between an increase in contrast, but also noise. 


\section{Part II | Dual-energy CT in stroke imaging}

The low keV monoenergetic reconstructions have an increased enhancement of the tissue attenuation curves due to the increased enhancement of iodine, resulting in a large increase in AUC. On the downside, with low keV reconstructions an increase in noise between frames is seen. Still, these effects result in a net increase in CNR for the low keV monoenergetic (40,50 and $60 \mathrm{keV}$ ) reconstructions as compared to the conventional CT images. These observations are supported by visual comparison of the CTP source images. The non-significant difference between CNR of 80 and $120 \mathrm{kVp}$ acquisitions is mainly due to the large variation in CNR for the $80 \mathrm{kVp}$ acquisitions. The monoenergetic reconstructions have a blotchy appearance, which is a characteristic of the reconstruction process.

The comparison of perfusion values between the monoenergetic and $120 \mathrm{kVp}$ reconstructions showed an effect in CBF, CBV and MTT values. This effect is probably due to differences in filtering between the different reconstructions and results in slightly increased MTT, and thus decreased CBF in comparison to $120 \mathrm{kVp}$. Even though the comparison of the perfusion values showed that sometimes there is a significant difference between reconstructions, the perfusion parameter maps from the monoenergetic reconstructions demonstrated a near equal quality for the CBV maps. This was also found by the observer; in most cases the quality of the CBV maps was similar. This is most likely due to the fact that estimation of the CBV is very robust, and is hardly influenced by differences in noise or enhancement. On the other hand, the quality of the MTT maps is substantially increased with all low keV monoenergetic. In future work, it might be interesting to use multiple monoenergetic reconstructions for the perfusion analysis.

The second qualitative evaluation showed that there was a significant increase in quality of the $50 \mathrm{keV}$ perfusion parameter maps as compared to perfusion parameter maps of the clinical standard of $80 \mathrm{kVp}$. Both observers came, independently from one another, to the same conclusion. The CBV estimates were qualitatively much better for the 50 $\mathrm{keV}$ compared to the $80 \mathrm{kVp}$. The $80 \mathrm{kVp}$ images need to be filtered with a relatively large kernel to be able to estimate the CBF and MTT accurately hampering the estimation of the CBV. For both reconstructions, the MTT remains hard to estimate, which was indicated by the observers as being a rather noisy perfusion map. However, the increase in enhancement with the $50 \mathrm{keV}$ did improve the quality significantly. This gain in image quality of the perfusion parameter maps can possibly contribute to the detection of smaller infarctions, imaged with equal or lower radiation and iodine contrast doses. 
This study has several limitations. First, we did not have CTP acquisitions of the same patient at 80 and $120 \mathrm{kVp}$, which because of ethical objections could not be performed. Therefore, it was not possible to do a direct comparison of the $80 \mathrm{kVp}$ and $120 \mathrm{kVp}$ reconstructions, but we feel that the chosen methodology still allows a comparison of the different reconstructions. Secondly, instead of focusing on infarct volumes, we focused on the overall (image) quality of the perfusion maps. This was done because a fair comparison of infarct volumes requires optimizing threshold values per protocol, which is not something straightforward and was therefore omitted. Thirdly, by using completely randomized datasets for the observers it was not possible to determine inter-rater agreement. We reported individual results only. Lastly, we have used thickslice reconstructions ( $5 \mathrm{~mm}$ ), but have not looked at thin-slice reconstructions, which can influence CNR and SNR and thus might increase the quality of the perfusion parameter maps. However, we have used a slice thickness which is used in most clinical settings and we have used the same reconstruction parameters for both patient groups, so we did not introduce a bias in this study.

In conclusion, this study showed that the quality of low keV monoenergetic CT perfusion images is superior to the quality of conventional 80 or $120 \mathrm{kVp}$ CT images. In particular, the $50 \mathrm{keV}$ reconstructions can significantly improve the quality of perfusion maps. 



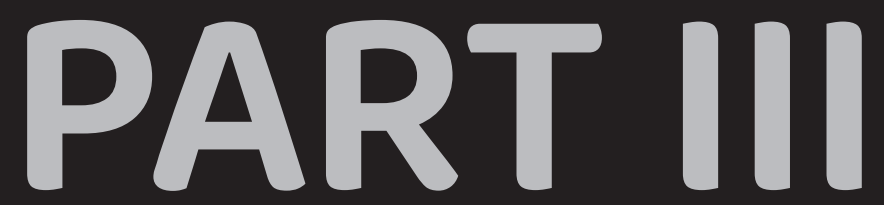

CT PERFUSION IN STROKE IMAGING 



\title{
CHAPTER 8
}

\section{EFFECTS OF ACQUISITION INTERVAL ON CT PERFUSION ANALYSIS}

\author{
Based on
}

Fvan Ommen, F Kauw, E Bennink, JW Dankbaar, MA Viergever and HWAM de Jong (2019). Effect of prolonged acquisition intervals for CT-perfusion analysis methods in patients with ischemic stroke. Medical Physics, 46:3156-3164.

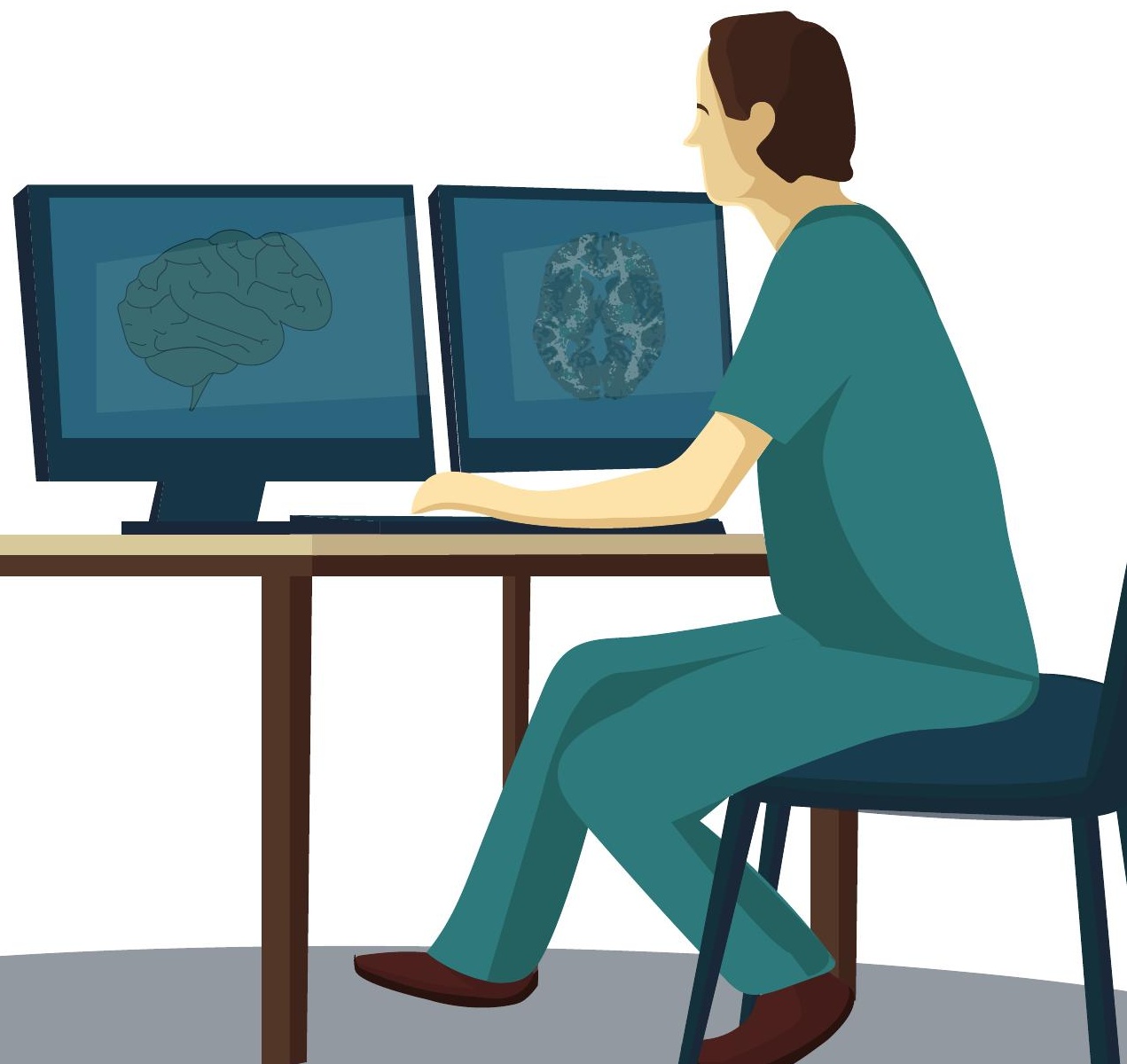


Part III | CT Perfusion in stroke imaging

\section{Abstract \\ Objectives}

The limited axial coverage of many CT scanners poses a high risk on false negative findings in cerebral CT perfusion (CTP) imaging. Axial coverage may be increased by moving the table back and forth during image acquisition. However, this method often increases the acquisition interval between CT frames, which may influence the CTP analysis. In this study, we evaluated the influence of different acquisition intervals on quantitative perfusion maps and infarct volumes by analyzing patient data with three CTP analysis methods.

\section{Methods}

CTP data from 25 patients with ischemic stroke were used for this study. The acquisition interval was synthetically reduced from 1 to 5 seconds before calculating perfusion values, which included cerebral blood flow (CBF), cerebral blood volume (CBV), and mean transit time (MTT). The color scaling of the perfusion was scaled such that the mean perfusion value had the same color-coding as the mean perfusion in the 1 second reference. Also, infarct core and penumbra volumes (summary map) were calculated using default thresholds of CBV and relative MTT (rMTT). The original, 1-second acquisition interval scan served as the reference standard. A commercial block-circulant singular value decomposition (bSVD) based method (ISP; Philips Healthcare), a non-commercial bSVD method, and a non-linear regression (NLR) model-based method were evaluated.

\section{Results}

CBV values generated with bSVD and NLR were not significantly different from the reference standard, while ISP showed significant differences for acquisition intervals of 3 and 4 seconds. MTT and CBF values generated with bSVD and ISP were significantly different for all acquisition intervals, whereas NLR did not show any significant differences. Calibrated perfusion maps were able to distinguish healthy from infarcted tissue up to an acquisition interval of 5 seconds for all methods. The infarct core volumes were significantly different for acquisition intervals of 2 (NLR) and 3 seconds (bSVD and ISP) or greater. For the penumbra volumes, NLR showed no significant differences, while bSVD and ISP showed significant differences for the 5-second interval and for all intervals, respectively. Visual inspection of the summary maps indicated minor differences between the reference standard and acquisition intervals of 4 seconds or less (ISP) and 5 seconds or less (bSVD and NLR).

\section{Conclusions}

Altering the acquisition interval may introduce a bias in the perfusion parameters. Calibration of the visualization of the perfusion maps with increasing acquisition intervals allowed distinction between healthy and infarcted tissue. Infarct volumes based on relative MTT can be influenced by the acquisition interval, but visual inspection of the summary maps indicated minor differences between the reference standard and acquisition intervals up to 4 (ISP) and 5 seconds (bSVD and NLR). Taken together, axial coverage can be increased by prolonging the acquisition interval up to 5 seconds depending on the perfusion analysis. 


\section{Introduction}

CT imaging is used worldwide to identify presence and potential causes of ischemic stroke in patients with suspected stroke[11,133]. CT imaging is quick, widely available, cost-effective, and has fewer contraindications than MRI, which makes CT the preferred imaging modality for the acute stroke setting. The acute stroke imaging protocol usually includes non-contrast CT, CT perfusion (CTP) and CT angiography imaging[134].

A dynamic contrast-enhanced CTP scan is typically an acquisition of one minute that monitors the wash-in and wash-out of the iodinated contrast agent in the brain parenchyma. A number of perfusion parameter maps can be inferred from CTP by using kinetic tracer analysis. These parameter maps give a quantitative insight into the perfusion status of the brain and are often used for clinical decision making as they can differentiate between healthy, salvageable (penumbra) and lost (infarct core) brain tissue[14,99].

The accuracy of the CTP analysis may depend on the number of frames that are acquired per series. In current stroke imaging protocols, frames are typically acquired every 1 or 2 seconds. It is hypothesized that more frames, which requires a shorter acquisition interval, would allow more precise monitoring of the contrast passage resulting in more accurate estimations of the perfusion parameters. However, the lowest achievable acquisition interval is limited because of dose and technical restrictions. Ideally, the CTP scan covers the entire brain, which is possible by using CT scanners that have a sufficient large axial coverage. However, the majority of CT scanners have insufficient axial coverage to cover the brain (usually $4 \mathrm{~cm}$ or less). This can cause false negative findings with CTP imaging, since the chosen cross-sectional images of the brain may not contain the ischemic area(s)[122,135,136]. Several methods (including Jog or Shuttle Mode and dual tracer injections) have been introduced to dynamically acquire an area that is larger than the original axial coverage. These protocols require the patient bed to be shifted or jogged in and out of the gantry. The time it takes for the table to move from one position to another, limits the lowest achievable acquisition interval. Jog Mode, which is available on the 64-slice IQon Spectral CT scanner (Philips Healthcare, Best, the Netherlands), yields a minimum acquisition interval of 3.4 seconds, whereas 1 or 2 seconds may be preferred for CTP imaging.

The acquisition interval of CTP imaging has been point of discussion in a number of papers, in which discordant findings were presented. Wintermark et al.[131] showed that acquisition intervals of up to 4 seconds could be reached without influencing the quantitative accuracy of CTP by maintaining the signal-to-noise ratio by increasing the amount of contrast agent. However, a number of studies observed opposing findings[137-139]. 
For instance, Wiesmann et al.[138] found that the acquisition interval could not be prolonged without compromising the image quality beyond 3 seconds. Similarly, Kämena et al.[137] recommended an interval of 2 seconds or lower, and Abels et al.[139] even recommended a maximum interval of 1 second. Instead of a fixed acquisition interval, Cao et al.[140] suggested the use of irregular sampling, since the monitoring of the bolus passage is more important than the remaining tail of the bolus. A possible explanation for these differences between studies is the observed differences in noise levels, due to the differences in acquisition parameters ( $k \vee p, m A s$, etc.). Interestingly, the reported studies also used different perfusion analysis methods, which may also explain the large differences in the response to prolonged acquisition intervals. Several CTP analysis methods exist such as block-circulant singular value decomposition (bSVD). In addition to commonly used analysis methods, we have introduced a non-linear regression-based perfusion analysis method (NLR), which is able to estimate perfusion parameters maps in a flexible and robust manner in response to noise, tracer delay, and truncation[17]. Therefore, NLR is hypothesized to sustain longer intervals than conventional methods.

In an effort to study the feasibility, reliability and accuracy of Jog Mode in CT perfusion imaging with equal contrast bolus volume, the purpose of this study is to assess the effects of increased acquisition intervals on quantitative perfusion parameters and infarct volumes. Three CTP analysis methods were evaluated: a commercial perfusion analysis method (ISP; Brain Perfusion, Intellispace Portal 9, Philips Healthcare, Best, the Netherlands), a non-commercial block-circulant singular value decomposition method (bSVD)[141] and the NLR method.

\section{Materials and Methods}

\section{Patient data}

For this study, 25 patients with ischemic stroke, who participated in the Dutch acute stroke study[132] (DUST), were randomly selected. All DUST participants gave informed consent for the use of their clinical and imaging data. In order to compare the performance of the perfusion analysis methods across different acquisition intervals, CTP data was collected. CTP data were originally acquired at an acquisition interval of 1 second, an axial coverage of at least $60 \mathrm{~mm}$, a tube voltage of $80 \mathrm{kVp}$ and an exposure of $75 \mathrm{mAs}$. In terms of dose levels, this protocol is in accordance with current stroke imaging protocols (acquisition interval of 2 seconds, $80 \mathrm{kVp}$ and $150 \mathrm{mAs}$ ). As it would be unethical to perform multiple CTP acquisitions on every single patient, we removed frames from the original dataset to mimic different acquisition intervals[131]. Hence, to acquire a dataset with an acquisition interval of 2 seconds, one frame was removed from every two frames. For the 3-second interval, two frames were removed from every three frames, and so forth up to an interval of 5 seconds. 
In this way, the dose, and thus the noise, remained stable for every frame, whereas the dose of the total CTP acquisition was reduced. The original dataset with an acquisition interval of 1 second was used as the reference standard. In Figure 8.1, examples of gray matter time-attenuation curves (TACS) are shown illustrating the effect of reducing the acquisition interval on the attenuation curve.
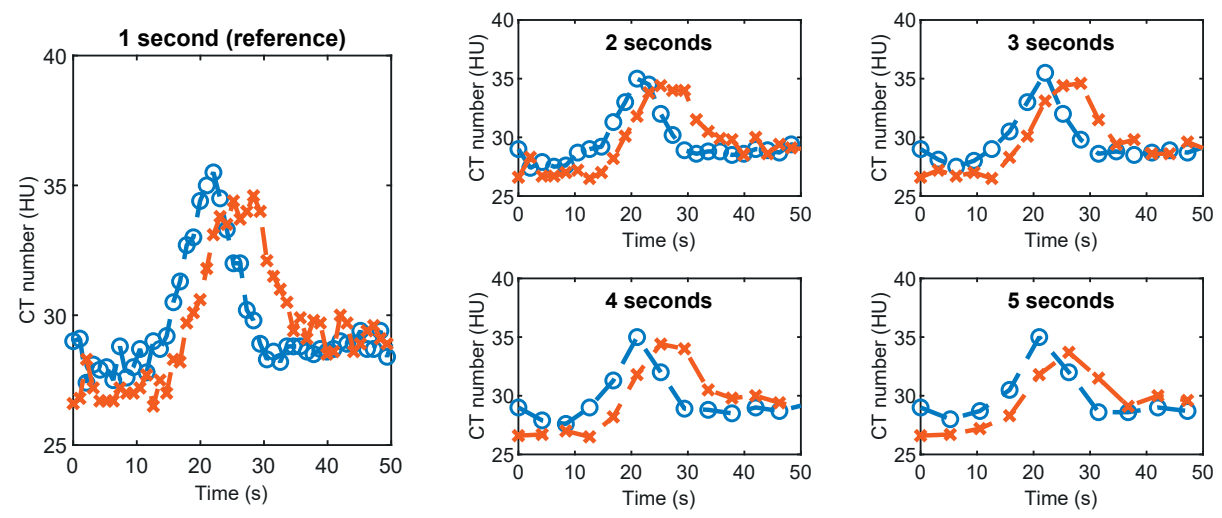

Figure 8.1 | Example time-attenuation curves of gray matter, illustrating the effect of reducing the acquisition interval on the attenuation curve. The blue circles illustrate an example in which the shape of the TAC is preserved with longer acquisition intervals, whereas the red crosses show a TAC which is slightly shifted in time and in which the shape is altered by the longer acquisition intervals.

\section{Pre-processing}

We corrected for motion by 3D rigid registration on the skull, which was done using the registration software package Elastix[129]. For the bSVD and NLR methods, the CTP images were filtered with a bilateral filter, explained in detail by Bennink et al.[17]. A target standard deviation of the noise of 0.75 Hounsfield units was used for the filter. As for the commercial method, the CTP scans were filtered using proprietary methods which are included in the ISP software.

\section{Perfusion analysis}

Tissue time-attenuation curves can be regarded as convolutions of the arterial input function (AIF) with a tissue-specific impulse response function (IRF). The shape of the IRF is determined by the different perfusion parameters (i.e. CBF, CBV, MTT and tracer delay). CTP analysis is a so-called inverse problem, since the IRF has to be derived from the measured TAC and AIF:

$$
\operatorname{TAC}(t)=\operatorname{IRF}(t) * A I F(t) .
$$


Non-linear regression (NLR) deconvolution

The NLR method is a non-linear model-based approach to solve this inverse problem. An initial guess of a block-shaped IRF is used for the convolution, after which a residual error $\varepsilon(t)$ is calculated between the estimated TAC and the measured TAC:

$$
T A C_{\text {est }}(t)=I R F_{\text {est }}(t) * A I F(t)+\varepsilon(t)
$$

Non-linear regression is then used to iteratively update the IRF estimate to minimize the residual error. From the estimated IRF, the perfusion parameters can be calculated as shown in the article by Bennink et al.[17].

(Block-circulant) singular value decomposition deconvolution

The convolution described can be written as a matrix operation: $b=A x+\varepsilon$, in which $b$ is the TAC and $A$ is a matrix containing circular shifted versions of the AlF. Vector $x$ is the IRF and vector $\varepsilon$ is the residual error. To get an estimate for $x$, matrix $A$ has to be inverted. In both the commercial method and in bSVD, singular value decomposition is used to find the inverse of $A$. In our bSVD implementation, noise is suppressed in the resulting IRF by removing the least significant eigenvectors until $x$ has an oscillation index below a certain threshold. In this study, acquisition interval specific oscillation indexes were used (1s: 0.035, 2s: 0.095, 3s: 0.17, 4s: 0.20, and 5s: 0.32). These values were determined by calculating the Pearson correlation between the input and observed MTT values at different oscillation indexes in simulated artificial data. For this purpose, an updated version of the anthropomorphic phantom by Riordan et al.[142] was used. The CBF is estimated from the maximum value of vector $x$ and the CBV by calculating the ratio between the $A I F(t)$ and $T A C(t)$. The MTT is calculated from $M T T=\frac{C B V}{C B F}$. The "time insensitive" method used in ISP (Brain Perfusion), which was used in this study, is based on bSVD.

\section{Selection AlF and venous output function}

In ISP, the TAC with the largest attenuation enhancement for the AIF and venous output function were automatically selected on the 1-second reference CTP image. The location of the AIF and venous output function was stored for each patient and used for all acquisition intervals, and also for the bSVD and NLR methods.

\section{Summary maps}

A summary map uses the quantitative perfusion parameters (CBF, CBV and MTT) to estimate the infarct core, i.e. irreversible damaged brain tissue, and the penumbra, which reflects a conceptual area that may still be salvaged if recanalization of the occluded vessel is achieved in time. For the generation of the summary maps CBV and the relative MTT (rMTT) was used. The rMTT is calculated by dividing the MTT values in the ipsilateral hemisphere by the MTT value of the contralateral hemisphere. Different thresholds for the determination of infarct core and penumbra were used across the different perfusion analysis methods (Table 8.1). 
Table 8.1 | The used thresholds for the calculation of the infarct core and penumbra volumes for the different perfusion analysis methods.

\begin{tabular}{lll}
\hline & Infarct core & Penumbra \\
\hline bSVD & CBV $<0.80$ & CBV $>0.80 \&$ rMTT $>1.30$ \\
ISP & CBV $<1.70$ & CBV $>1.70 \&$ rMTT $>1.85$ \\
NLR & CBV $<0.90$ & CBV $>0.90 \&$ rMTT $>1.35$ \\
\hline
\end{tabular}

The thresholds were calculated by maximizing the Dice overlap coefficient[143] between the estimated infarct core and penumbra on the one hand, and the known volumes for those regions in the updated anthropomorphic digital phantom[142] sampled at 1 second acquisition interval, on the other hand.

\section{Image analysis}

For the analysis of the CTP data, two identical circular regions of interest (ROIs) were drawn in each hemisphere: one in the gray matter (GM) and one in the white matter (WM). The position and size of the ROls was the same for the 25 datasets. These ROIs were used to calculate the average perfusion parameters (CBV, CBF and MTT) for the patient group. The color scaling of the perfusion was scaled such that the mean perfusion value had the same color-coding as the mean perfusion in the 1 second reference. In addition, the volumes of the infarct core and penumbra were measured.

\section{Statistical analysis}

The CBF, CBV and MTT values, and the core and penumbra volumes across the different acquisition intervals were compared to the 1-second acquisition interval (reference) dataset. Differences were evaluated with the Wilcoxon signed-rank test. The significance level was set to 0.05. Statistical analysis was performed in MATLAB (MathWorks, Natick, MA, USA).

\section{Results}

\section{Quantitative perfusion values}

Gray matter mean perfusion values (CBF, CBV, and MTT) for the different acquisition intervals are shown in Figure 8.2. The CBF, CBV and MTT values that were significantly different from the reference standard are marked with an asterisk. ISP showed that the CBV estimates were rather constant, except for the acquisition interval of 4 seconds. For bSVD, CBV estimates did not show significant differences at all acquisition intervals. CBF and MTT value generated by DSVD and ISP were significantly different from the reference standard at every acquisition interval, whereas NLR did not show any significant difference in comparison to the 1-second reference. 


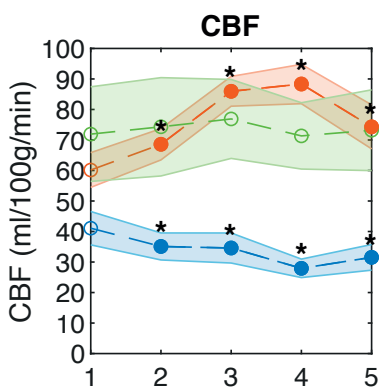

Acquisition interval (s)

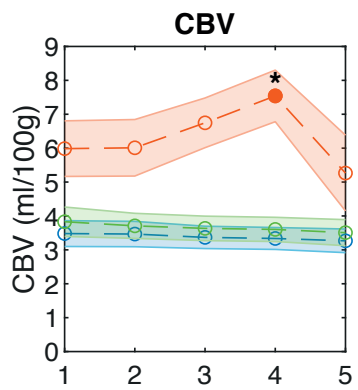

Acquisition interval (s)

$$
\text { - }- \text { - bSVD - } \theta-\text { ISP }-\ominus-N L R
$$

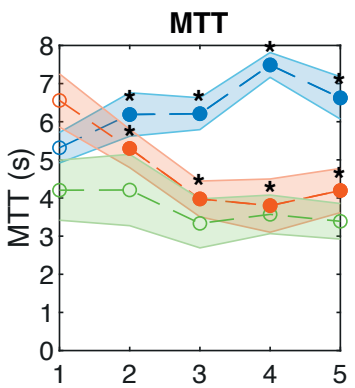

Acquisition interval (s)

Figure 8.2 Mean CBF, CBV and MTT values with 95\% confidence interval for gray matter for bSVD, ISP and NLR. The values that are significantly different from the 1-second acquisition interval (reference) are filled and marked with an asterisk.

In Figure 8.3, the mean CBF, CBV and MTT values of the white matter are shown. The observed trends with the white matter were similar to those of the gray matter. The CBF, CBV and MTT values that were significantly different from the reference standard are marked with an asterisk. The perfusion analysis methods showed constant CBV values, except for ISP, for which the 3 and 4 second intervals differed significantly from the reference standard. DSVD and ISP showed CBF and MTT values that were significantly different from the reference standard, whereas NLR showed no significant differences.
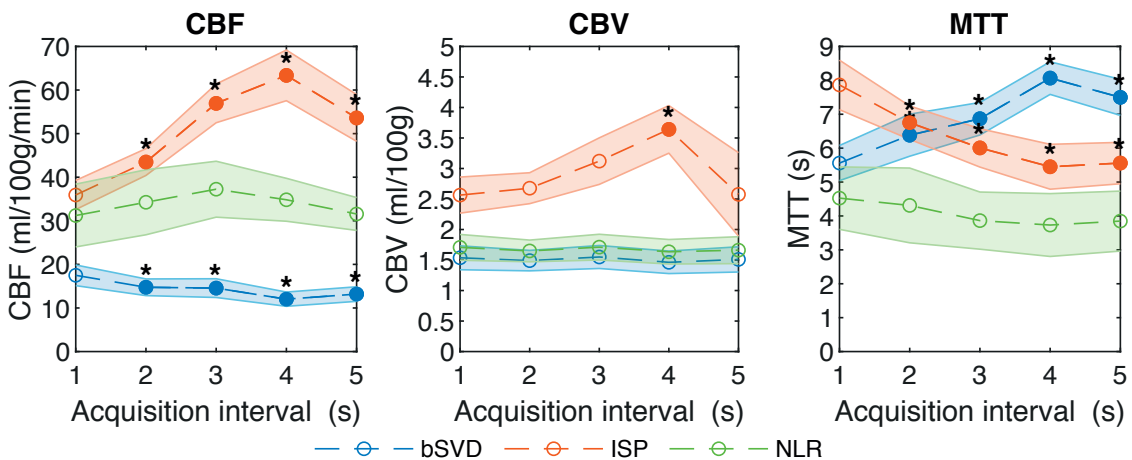

Figure 8.3 | Mean CBF, CBV and MTT values with 95\% confidence interval for white matter for bSVD, ISP and NLR. The values that are significantly different from the 1-second acquisition interval (reference) are filled and marked with an asterisk.

\section{Perfusion maps}

The trends observed in Figure 8.2 and Figure 8.3 can be used for calibrating the colorcoding range of the perfusion maps. To illustrate this for one patient, we have generated CBF maps, which are displayed in Figure 8.4. These calibrated CBF maps are able to distinguish between areas of healthy and infarcted tissue up to 5 seconds. 
Hardly any differences between the CBF maps for the three CTP analyses were observed. Similarly, we generated calibrated CBV maps, which are shown in Figure 8.5. The CBV maps were quite similar across the different acquisition intervals. The generated MTT maps are shown in Figure 8.6. Compared to the reference standard, MTT maps generated with bSVD were relatively noisy for the 4 and 5 second acquisition intervals, whereas ISP and NLR maps are more in agreement with the reference standard.

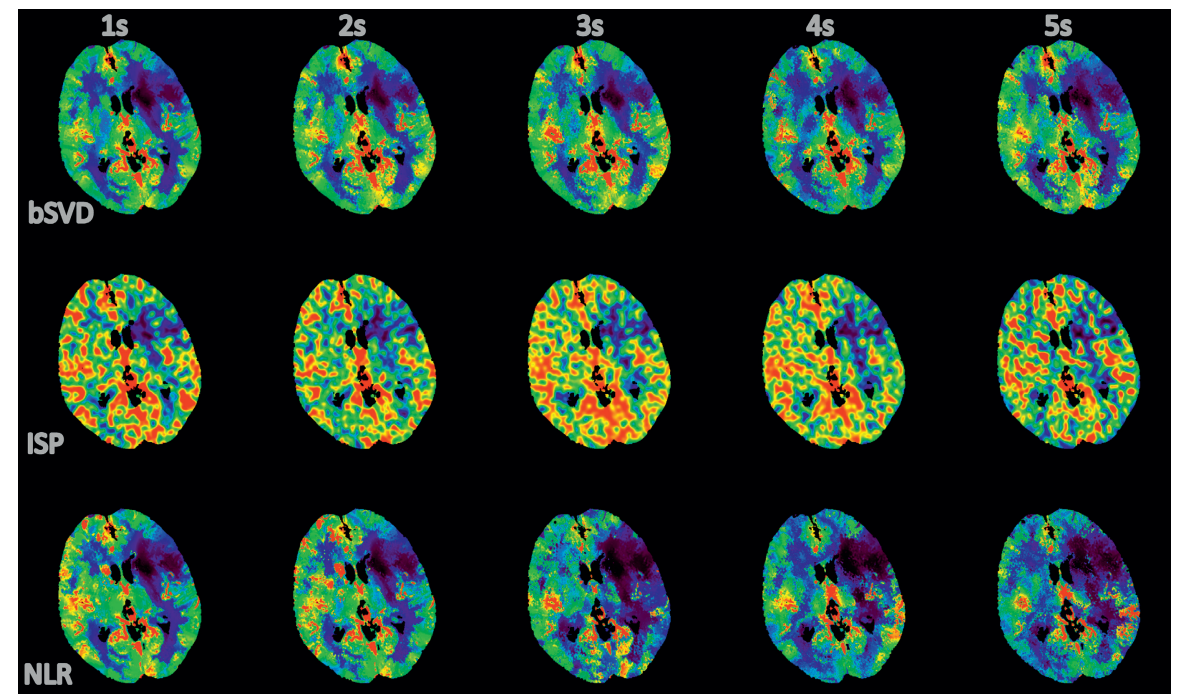

Figure 8.4 | An example of calibrated CBF maps generated using bSVD, ISP, and NLR as a function of acquisition interval.

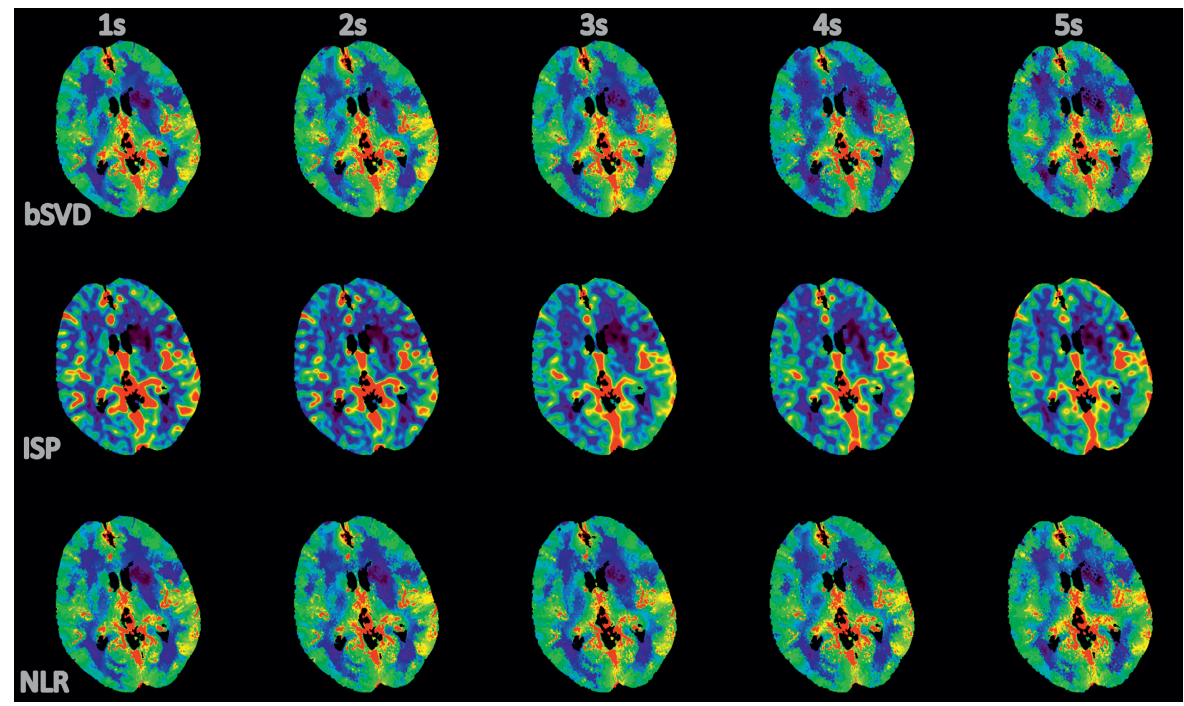

Figure 8.5 | An example of calibrated CBV maps generated using bSVD, ISP, and NLR as a function of acquisition interval. 


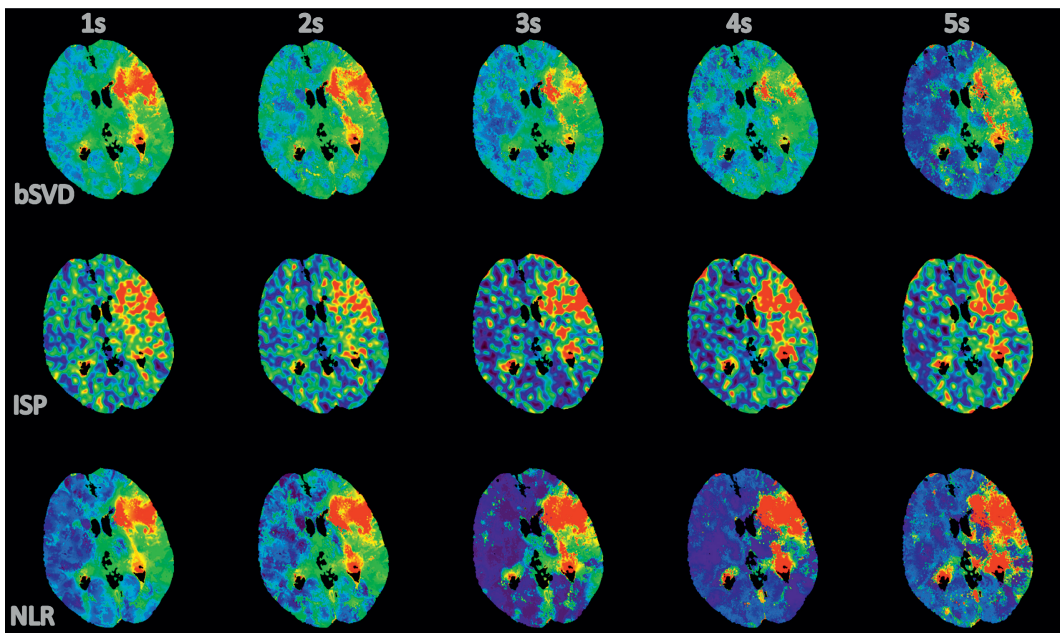

Figure 8.6 | An example of calibrated MTT maps generated using bSVD, ISP, and NLR as a function of acquisition interval.

\section{Summary maps}

The summary maps were generated for every acquisition interval. Example summary maps are shown in Figure 8.7. Visually, the summary maps generated with bSVD were rather similar to the reference summary map. Summary maps generated with ISP were similar up to an acquisition interval of 4 seconds, but the 5 -second acquisition interval summary map had a significant increase in core volume (red) than the reference standard. Still, the 5-second acquisition interval summary map looks quite similar to the reference standard. Summary maps generated with NLR were fairly constant.

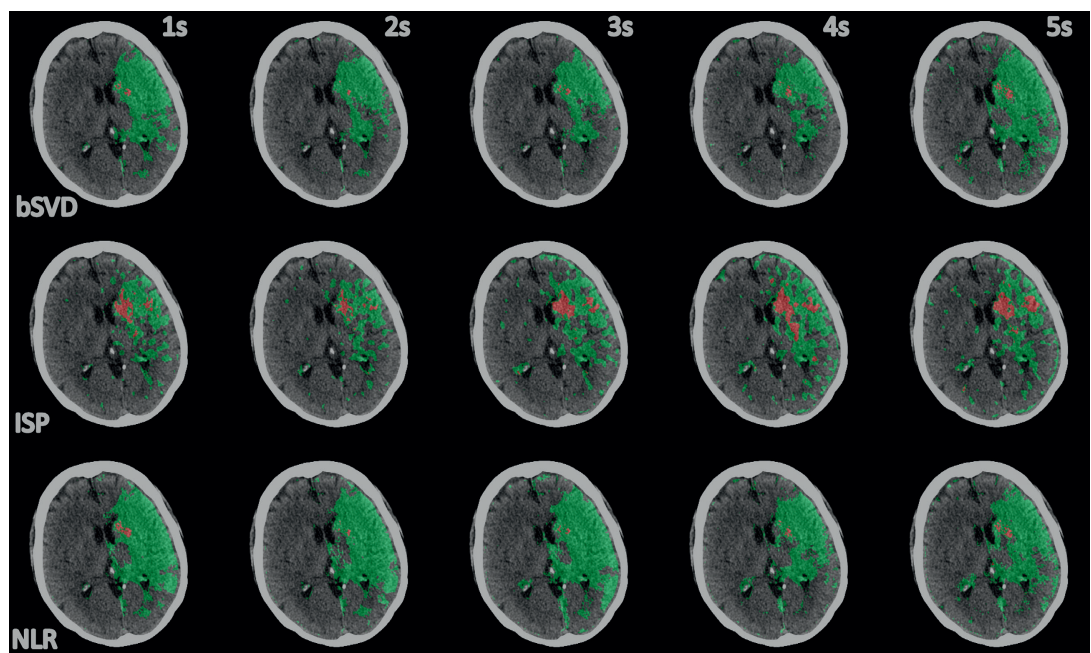

Figure 8.7 | Summary maps of bSVD, ISP, and NLR as a function of acquisition interval. The infarct core is labeled red, the penumbra labeled green. This is the same patient as shown in Figure 8.4, 8.5, and 8.6. 
Average core and penumbra volumes generated by the three CTP analysis methods are shown in Figure 8.8. Core volumes generated by bSVD and NLR show a monotonic significant increase with increased acquisition interval. Core volumes generated by ISP shows a monotonic decrease in volume, with a large significant increase for the 5 second volume. The core volume was significantly different from the reference standard at 3, 4 and 5 seconds intervals. The penumbra volumes generated by bSVD and NLR did not show any significant differences, except for the penumbra volume generated with bSVD on the 5-second interval. Penumbra volumes generated with ISP were all significantly larger than the reference standard.
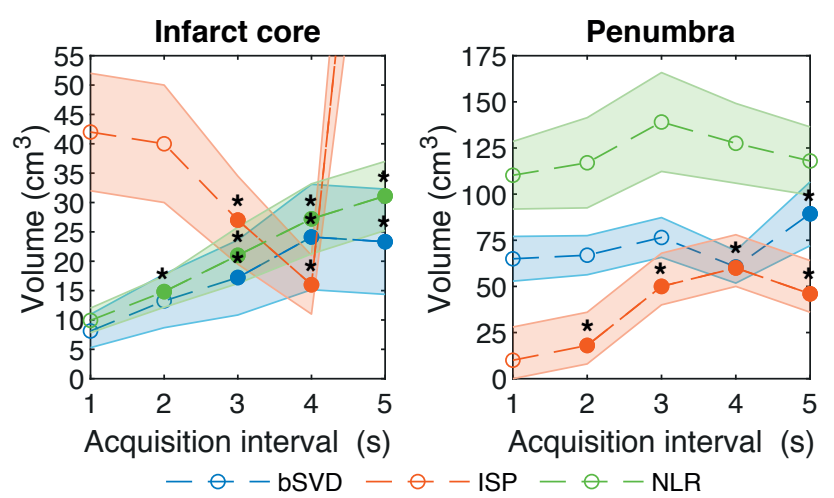

Figure 8.8 Mean volumes $\left(\mathrm{cm}^{3}\right)$ with 95\% confidence interval of the infarct core and the penumbra. If the volume was significantly different from the reference standard it is filled and marked with an asterisk. The 5 second ISP core volume was over $100 \mathrm{~cm}^{3}$ and therefore not included in the figure.

\section{Discussion}

In this study, the response of three perfusion analysis methods to different acquisition intervals was investigated by analyzing CTP data from 25 patients with ischemic stroke. ISP overestimated the CBF and CBV and underestimated MTT as compared to the 1-second reference standard in the gray and white matter. The 5-second dataset, however, deviated from this trend. This trend was already observed by Wintermark et al.[131], when using a $40 \mathrm{ml}$ iodinated contrast bolus. Wintermark et al., however, did not observe significant differences up to an acquisition interval of 3 seconds, whereas in our dataset we observed significant differences at 2 seconds, except for the CBV values. There are two main causes of the observed differences. Firstly, Wintermark et al. acquired at $80 \mathrm{kVp}$ and $120 \mathrm{mAs}$ every 0.5 seconds, whereas we acquired CTP Data at $80 \mathrm{kVp}$ and $75 \mathrm{mAs}$ every second. As a consequence, our images are significantly noisier. Secondly, Wintermark et al. used the so-called "arrival time sensitive" algorithm, whereas we used the newer "arrival time insensitive" algorithm, which might potentially lead to observed differences. 
The observed differences between bSVD and ISP were caused by differences in the used oscillation index, filtering and post-processing. As hypothesized, perfusion values generated with NLR were not significantly different across the different acquisition intervals indicating that NLR estimates perfusion parameters in a more robust manner. This can probably be explained by the fact that most TACs have long enough MTTs maintaining the fit of the curve. As a result, NLR generated accurate estimates even with prolonging acquisition intervals. We did observe a large variance in the CBF and MTT values of NLR compared to the other methods, which can be attributed to the fact that we saw large inter-patient differences, but not large differences with increasing acquisition intervals within a patient. In addition, the large variance is due to regularization of the TACS, which makes the estimates of NLR more realistic, but this results in an increase in variance of the CBF. bSVD and ISP generated significantly different CBF and MTT values for all acquisition intervals compared to the reference standard. However, because a similar acquisition interval-dependent trend was observed in both the gray and white matter, the perfusion maps could be appropriately generated by calibrating the colorcoding based on the observed values. This suggests that the over- or underestimation of the perfusion variables by the CTP analysis methods is a systemic bias dependent on acquisition interval and that the distinction between healthy and infarcted tissue can still be made for acquisition intervals of up to 5 seconds. The approach of calibrating the color-coding range, however, is not validated on a separate independent dataset and thus needs further evaluation.

bSVD and NLR generated infarct core volumes, which increased with increasing acquisition intervals is mainly due to an increase in remote voxels misclassified as core due to noise. This issue can possibly be solved by introducing a post-processing filter step, which removes these spurious pixels and subsequently reduces the infarct core volumes for prolonged acquisition intervals. For ISP, a decrease in core volume was observed with increasing interval, mainly due to the overall increase of the CBV values and could possibly be solved by scaling the core thresholds. ISP generated significantly higher penumbra volumes with increasing acquisition intervals, which can be attributed to the number of remote pixels misclassified as penumbra. For bSVD and NLR, only a minor change in penumbra volumes with increasing acquisition interval was shown, which can be explained by the slight increase in noise in the summary maps due to noise. Even though changes in infarct core and penumbra volumes are observed, the summary maps show that with acquisition intervals of at least 4 (ISP) and 5 (bSVD and NLR) seconds it is still feasible to distinguish between infarcted and healthy tissue. 
This study has several limitations. Firstly, we did not have any validation of the true infarct core and penumbra volumes in the patient data. However, as we were only interested in the differences that arise from using different acquisition intervals, we do not think that this limitation impacts our conclusions. Still, comparison with a reference standard such as DWI-MRI would be needed in future studies before these methods can be safely used in stroke care. Secondly, to create CTP datasets with increasing acquisition intervals, we deleted images from a 1-second reference dataset. It would have been ideal if we could have scanned every patient with the five different acquisition intervals. However, this approach would not be ethical and we feel that our used methods simulate repeated scanning on different acquisition intervals appropriately. Thirdly, there are no standardized thresholds available for bSVD and NLR to calculate infarct volumes. To quantify any bias in volume due to the longer acquisition intervals, we decided to calculate thresholds optimal for all methods for this dataset. The used core and penumbra volume thresholds are not verified for a large variety of patient groups, and there is no justification for the method used to generate the volumes. The purpose of the generation of the volumes was mainly to illustrate that infarct volumes are reliable with longer acquisition intervals. Lastly, to evaluate Jog Mode we looked at acquisition intervals of up to 5 seconds, but we did not include the movement of the table, which causes a time shift in the TACs of the top and bottom half of the brain. However, since the used methods are insensitive to bolus arrival time by design, we feel that this time shift has had little effect on the generated perfusion parameters.

In conclusion, altering the acquisition interval may introduce a bias in the perfusion parameters. However, calibration of the visualization of the perfusion maps with increasing acquisition intervals allowed distinction between healthy and infarcted tissue. Even though infarct volumes based on relative rMTT can be influenced by the acquisition interval, visual inspection of the summary maps indicated minor differences between the reference standard and acquisition intervals up to 4 (ISP) and 5 seconds (bSVD and NLR). Taken together, dependent on the used CTP analysis method, axial coverage can be increased by prolonging the acquisition interval up to 5 seconds without affecting the generation of accurate perfusion parameters and infarct volumes. 



\title{
CHAPTER 9
}

\section{THIN-SLICE CT PERFUSION FOR DETECTION OF SMALL-VOLUME INFARCTION}

\author{
Based on
}

F van Ommen, JW Dankbaar, F Kauw, E Bennink and HWAM de Jong. Thin-slice CT perfusion for detection of small-volume infarction using bilateral filters. In preparation.

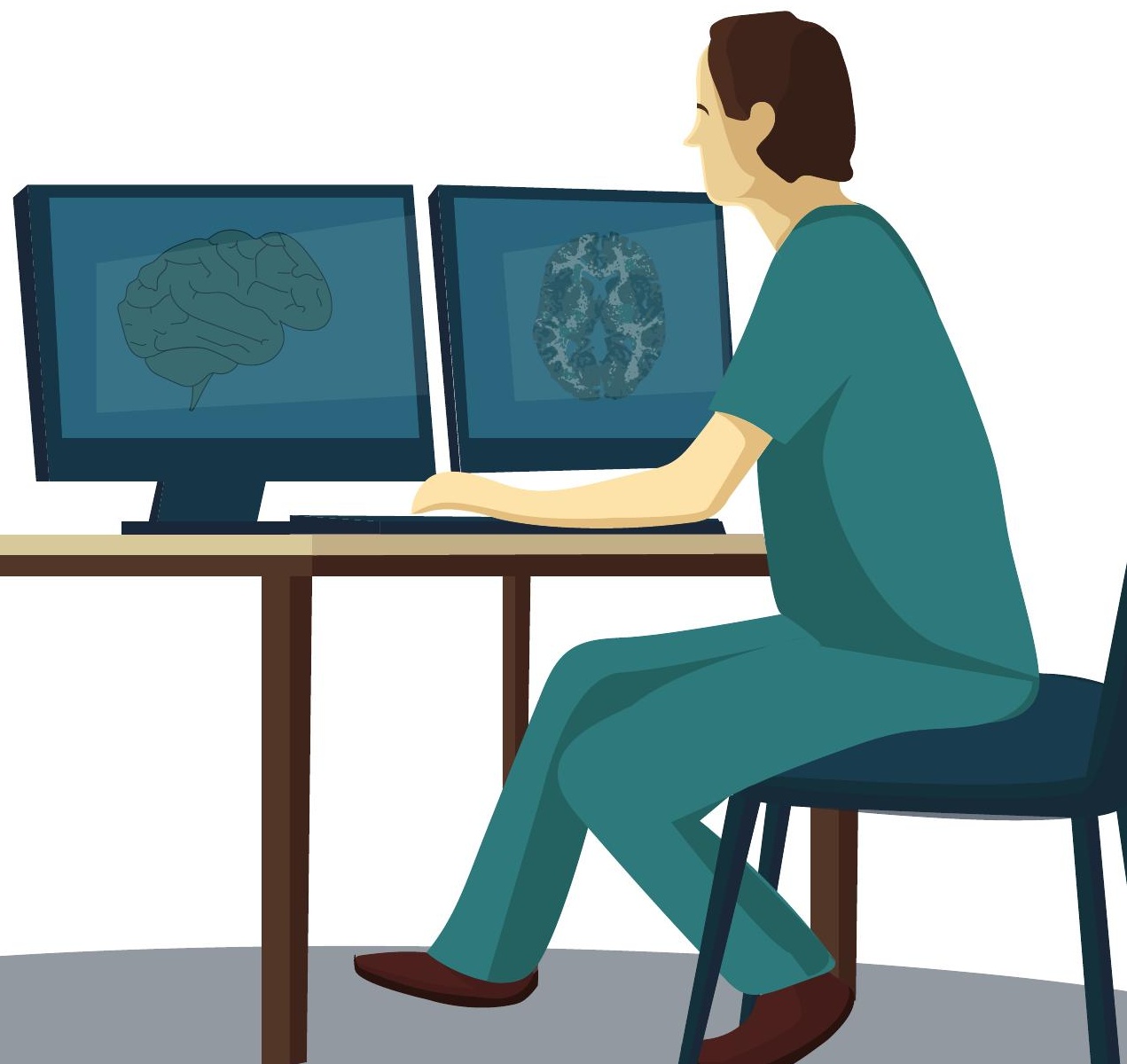


Part III | CT Perfusion in stroke imaging

\section{Abstract \\ Objectives}

Small-volume infarctions are hard to detect with standard thick-slice CT perfusion (CTP) protocols. Thin-slice CTP might increase detection of small-volume infarcts, but increases image noise requiring robust filtering approaches. We evaluated the performance of two bilateral filters for the detectability of small-volume infarction.

\section{Methods}

A digital anthropomorphic phantom and a patient cohort who underwent CT perfusion admission scanning and subsequent DWI were evaluated. Phantoms and patients were filtered using a time-intensity profile similarity bilateral filter (TIPS) and a bilateral filter guided by a principal component image (pBF). Phantoms were compared to ground truth values for cerebral blood flow (CBF) and CT number using the root-mean squared error (RMSE). Absolute CBF, cerebral blood volume (CBV) and mean transit time (MTT) values are measured in the gray matter (GM) and white matter (WM) and in the ground truth infarct. Contrast was measured using contrast-to-noise ratio (CNR) for GM-WM, GM-infarct and WM-infarct. For patients, perfusion values in GM, WM and GM-WM CNR were evaluated. Lastly, an observer evaluated the perfusion maps obtained with the different filters for presence and location of infarct, blinded for the DWI-MRI gold standard. Infarct location accuracy was assessed using infarct location match (ILM). ILM subgroup analyses were dichotomized by infarct volume on DWI. Diagnostic certainty and overall image quality were evaluated using a 5-point Likert scale.

\section{Results}

The phantom study showed that CBF-RMSE, CT number-RMSE, perfusion values and CNR were estimated with the highest accuracy by pBF. 58 patients were included in our patient study. pBF showed highest CNR for CBF and CBV GM-WM (1.24 and 1.57 vs TIPS: 0.69 and 0.80; $p<0.001)$. MTT GM-WM CNR was similar between filters. Infarct detection sensitivity was highest for pBF, but not significantly different (54.1 vs TIPS: 40.5\%; $p=0.063$ ). Specificity was highest for TIPS (100.0 vs pBF: 90.5\%; $p=0.500$ ). Overall ILM was significantly higher for pBF compared to TIPS (37.8 vs 30.0\%; $p=0.016$ ). Subgroup analysis showed significantly improved small-volume infarct overall-ILM for pBF compared to TIPS (38.9 vs 30.0\%; $p=0.016$ ). Small-volume infarct overall-ILM of pBF was significantly higher compared to TIPS (33.8 vs 25.0\%; $p=0.016$ ), but there was no difference in large-volume infarct detection. The diagnostic certainty and image quality was significantly higher for pBF (3.47 vs TIPS: 3.22; $p=0.022$ and 3.10 vS TIPS: 2.14; $p<0.001)$. 


\section{Conclusions}

To our knowledge, this was the first study on the performance of thin-slice CTP for detection of small-volume infarcts, indicating that more than half of these strokes are detectable especially if larger than $1 \mathrm{ml}$. Perfusion values are estimated more accurately and with higher contrast using guided bilateral filtering. While the detection of smallvolume infarctions remains difficult, infarcts could be detected with higher sensitivity and significantly higher diagnostic certainty and improved image quality using guided bilateral filtering than with the current state-of-the-art TIPS filter. 


\section{Introduction}

A CT perfusion (CTP) scan is a dynamic acquisition of the brain parenchyma, in which the wash-in and wash-out of iodinated contrast agent, is described by local timeattenuation curves (TACS). From these TACs, a set of perfusion parameters can be estimated; e.g. cerebral blood flow (CBF), cerebral blood volume (CBV) and mean transit time (MTT). These parameters can be used to visualize perfusion of the brain tissue in acute ischemic stroke patients and thereby assist in detection of areas of hypoperfusion and the differentiation between healthy, salvageable (infarct penumbra) and lost (infarct core) brain tissue regions. This information is important in stroke treatment decisions[14,108].

Small-volume infarctions ( $\leq 4.2 \mathrm{ml}[144])$ and posterior infarcts are clinically relevant but hard to detect with current CTP protocols[145]. These protocols utilize thick slices of 5 to $10 \mathrm{~mm}$ thickness to decrease the noise and limit the radiation dose of the CTP acquisition. By applying thick slices, smaller structures within such a slice are averaged resulting in strong partial volume effects. It has been suggested that thin-slice reconstructions can improve the detectability of small-volume infarcts[122], which make up about 15 - 25\% of all infarctions[146,147]. In thin-slice CTP, slice thicknesses of $\leq 1$ $\mathrm{mm}$ are utilized which results in high noise levels, and thus require more sophisticated image filters to reduce noise. There are many different filtering techniques available, e.g. Gaussian smoothing, masked smoothing[148], time-intensity profile similarity (TIPS) bilateral filtering[149] and guided bilateral filtering[17,150]. Thin-slice CTP has been evaluated with the TIPS bilateral filter in one study[151], ranking the image quality of the different filters and evaluating vessel visibility. However, to the best of our knowledge, the detectability of small-volume infarcts using thin-slice CTP in a clinical setting has not been investigated. In this study, the performance of thin-slice CTP for detection of small-volume infarctions was evaluated. To this end two different bilateral filters were implemented.

\section{Materials and Methods}

In bilateral filtering, as introduced by Tomasi and Manduchi for noise reduction of 2D images[152], both the Euclidean distance between neighboring voxels and the intensity difference between those voxels is used to replace each voxel in an image with a weighted average of the similar and neighboring voxels. A well-known extension of the bilateral filter for CTP is the TIPS bilateral filter, in which a net intensity difference is calculated as the sum of squared differences (SSD) between the TACs of two voxels[149]. The guided bilateral filter does not get its intensity differences from the source image directly, but uses an intermediate guide image to calculate the intensity pixel similarity measure. In the guide image, voxels who belong to the same tissue type typically have comparable values indicating a strong averaging between these voxels can be applied without losing contrast between tissues. 
The guide image of the guided bilateral filter can be calculated in a number of ways, e.g. by clustering voxels with similar TACS[150] or by averaging all time-frames of the CTP scan[17]. In this study, a principal component analysis (PCA) guide image was used. In PCA all TACs are decomposed into a series of components (curves) in which the first component contains the most variance, and thus contrast. Voxels belonging to the same tissue will have a comparable amount (or weight) of this first component in their TACs, which can be used as a guide image for the filter. This prevents averaging of different tissue types, and thus preserves contrasts between tissue regions.

Bilateral filter strength is controlled by two parameters. The first parameter controls the weight of the spatial (Euclidean) distance on the voxel averaging by the standard deviation $\left(\sigma_{d}\right)$, which determines at what distance the neighboring pixels are still considered to be close, i.e. $\sigma_{d}$ determines the number of neighboring voxels that are included in the weighted average. The second parameter is the standard deviation of the Gaussian range kernel $\left(\sigma_{r}\right)$, which in case of the TIPS filter determines up to which point the time-attenuation curves are still considered to be similar. For the guided bilateral filter it determines to what extend two voxel values in the guide image are still considered similar.

\section{Datasets}

Phantom

An anthropomorphic phantom with a small-volume infarct $(1.39 \mathrm{ml})$ was created based on the method described by Riordan et al.[142]. High resolution (0.5 mm isotropic) segmentations of a series of MR brain scans[153] were used to define CT-relevant tissue regions and vessels containing realistic CT numbers. Time attenuation curves describing contrast bolus passing were added to the different regions by convolving an artificial impulse response function[154] with an arterial input function (AIF). The high-resolution AIF was created by taking the AIFs of 50 registered CTP scans, which were interpolated to 0.1 second intervals, aligned, and averaged into one generic AIF. Spatially correlated noise was added to every time frame with the method described by Britten et al.[155]. Acquisition parameters were set in accordance with current clinical practice. 


\section{Patients}

This study was approved by the University Medical Center Utrecht institutional review board, and patients signed written informed consent. Inclusion criteria were: baseline thin-slice CTP, age $\geq 18$ years, presentation within 9 hours of symptom onset, no occlusion on admission CTA or an occlusion distal of A2 of the anterior cerebral artery, occlusion distal of M1/M2 bifurcation and/or occlusion of the posterior circulation (excluding the basilar artery) and follow-up MRI with DWI within 48 hours of baseline CTP. Exclusion criteria included unavailable thin-slice perfusion imaging and residual motion after motion correction. Patients were scanned on two CT scanners (Philips Healthcare, Best, the Netherlands); the acquisition and reconstruction parameters are shown in Table 9.1. A 40 (iCT) or 50 (IQon) ml contrast bolus (Ultravist-300 mg/ml, Bayer, Leverkusen, Germany) was injected with a flow of $6 \mathrm{ml} / \mathrm{s}$ followed by $40 \mathrm{ml}$ of saline at a flow of $6 \mathrm{ml} / \mathrm{s}$.

\section{CT perfusion}

Patient data were corrected for motion by 3D rigid registration on the skull using the registration software package Elastix[129]. Phantoms and patients were filtered with a TIPS bilateral filter[149], and PCA-guided bilateral filter (pBF). To allow a fair comparison between filters, parameter settings of the different filters were set to equalize the average coverage of their kernels, i.e. the median volume of each filter kernel has to be equal (Figure 9.1). The coverage of a filter kernel determines the noise variance in the output. The standard deviation of the range kernel for TIPS was set as suggested by the original paper to $\sigma_{r}=\sigma_{n}{ }^{2}$, in which $\sigma_{n}$ is the standard deviation of the noise in the CTP acquisition. The $\sigma_{n}$ in the CTP acquisition is calculated from the frames before the bolus arrival. For pBF, the range kernel uses the information that is in the guide image or first PCA component. The range kernel of the pBF was therefore scaled to the relative amount of variance in that component, which reflects the amount of information: $\sigma_{r}=4 \sigma_{n} \frac{1}{\sigma_{i}^{2}} \sum_{j=1}^{m} \sigma_{j}^{2}$, in which $\sigma_{i}^{2}$ is the variance in the guide image, $\sigma_{j}^{2}$ is the variance in the $j^{\text {th }}$ principal component, and $\sigma_{n}$ is the standard deviation of the noise in the CTP acquisition. Filtered images were subsequently analyzed with non-linear regression deconvolution to obtain parameter maps[17]. The same AIF and venous output function location was used for every filter with the phantom and patients. 
Thin-slice CT perfusion for small-volume infarction | Chapter $\mathbf{9}$

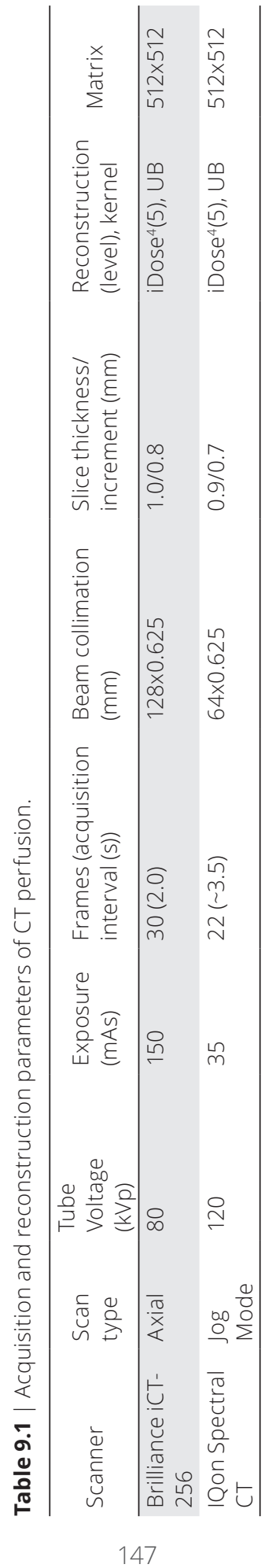




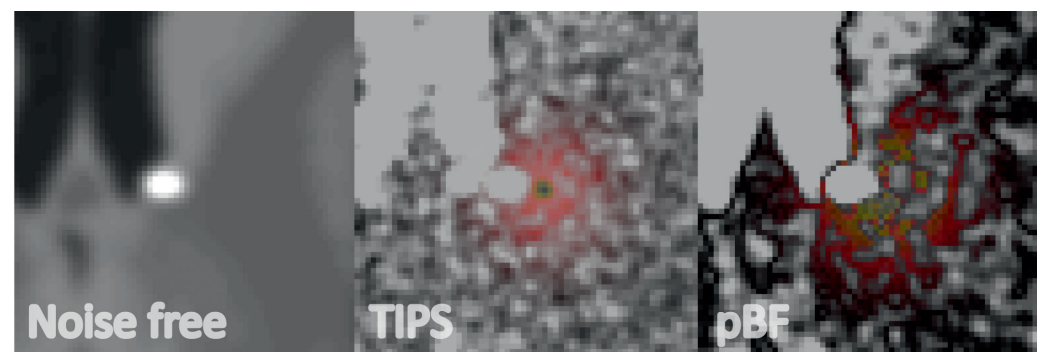

Figure 9.1 | Example kernels of the same white matter pixel from the different filters. In the noise free image a vessel (white spot), gray matter and white matter are clearly visible; these are structures that ideally should be preserved using the filters. Both filter kernels preserve the vessel by avoiding the structure in their filter kernel. The TIPS filter kernel is slightly more Gaussian shaped, whereas the pBF kernel is shaped more to the white matter. Both filter kernels are shown on a range kernel image for the pixel in the center, i.e. the sum of squared differences between the attenuation curves in case of TIPS, and the absolute difference between the values in the guide image for pBF. This shows that with TIPS the contrast between gray and white matter is lost.

\section{Study design}

Phantom study

Ten realizations of the anthropomorphic phantom were generated, and filtered with TIPS and pBF. To identify to what extend the filtering methods could reconstruct the noise free CTP phantom (ground truth), the root-mean squared error (RMSE) between the ground truth and the filtered CTP was calculated in all intracranial tissue pixels, in the gray and white matter, and in the infarct core. In addition, the RMSE between the CBF of the filtered images and the ground truth was calculated. The absolute CBF, CBV and MTT values are also calculated in regions of interest (ROIs) in the gray and white matter and the infarct core. Furthermore, a contrast-to-noise ratio (CNR) was calculated between the gray and white matter, between the gray matter and infarct core and between the white matter and infarct core. The CNR between two tissue regions is defined as:

$$
C N R=\frac{\mu_{1}-\mu_{2}}{\sqrt{\sigma_{1}^{2}+\sigma_{2}^{2}}}
$$

In which $\mu$ is the mean and $\sigma$ is the standard deviation of the perfusion value.

\section{Patient study}

For the patient study, similar to the phantom, the absolute CBF, CBV and MTT values were calculated in the gray and white matter, and a GM-WM CNR was calculated for gray-white matter differentiation. For the analysis of the perfusion maps, two identical circular ROls of $25 \mathrm{~mm}^{2}$ were drawn in each hemisphere, one in the gray matter and one in the white matter. In addition, a reviewer visually evaluated all patients. Each filtered scan was presented in a randomized and blinded fashion. An experienced neuroradiologist (JWD) evaluated each filtered CTP and corresponding perfusion maps for the detectability of infarction. Each CTP was scored on a binary scale for presence of cerebral infarction. 
Cerebral infarcts were binned by location using the anatomical division from the Alberta Stroke Program Early CT Score (ASPECTS)[92], posterior circulation ASPECTS (pc-ASPECTS)[93] and anterior cerebral artery flow territory (A1 = lower half and A2 = upper half). A five-point Likert scale was used to quantify diagnostic certainty and to score image quality of the perfusion maps as follows: 1, very poor; 2, suboptimal; 3, acceptable; 4, good and 5, excellent.

\section{Statistical analysis}

Summary statistics for RMSE, absolute perfusion values and CNR were all reported in mean and standard deviations and compared using a paired t-test with a Bonferroni correction for multiple comparisons. Observer results for infarct detection were evaluated using sensitivity, specificity, positive and negative predictive value (PPV and NPV) and accuracy. Sensitivity and specificity are compared using McNemar's tests. Regional infarct location match (ILM) between DWI and CTP was calculated as the percentage of true positive detections of infarction on CTP compared to the total of true positive infarctions on DWI in an anatomical region. ILM was compared for all regions and for the cortical regions (A1, A2, M1 - M6, insular cortex and superficial posterior cerebral artery), basal ganglia (internal capsula, nucleus lentiform and caudate nucleus) and posterior circulation (thalamus, cerebellum, pons and midbrain). Furthermore, a subgroup analyses of small $(\leq 4.2 \mathrm{ml})$ and large $(>4.2 \mathrm{ml})$ volume infarction based on the size of infarct on DWI for ILM was performed. $4.2 \mathrm{ml}$ was chosen because this is the volume of a sphere with diameter $20 \mathrm{~mm}$, similar to a small-volume infarct[144]. ILM was compared between filter techniques using McNemar's test. Diagnostic certainty and image quality was evaluated using a paired t-test. Statistical analyses were conducted in SPSS version 25.0 (IBM, Armonk, NY, USA).

\section{Results}

\section{Phantom}

In Figure 9.2, the RMSE of the filtered images and CBF are shown for all intracranial tissue, white and gray matter, and the infarct core. For all masks, TIPS showed a significantly higher RMSE for both CT numbers and CBF compared to pBF $(p<0.002)$. 


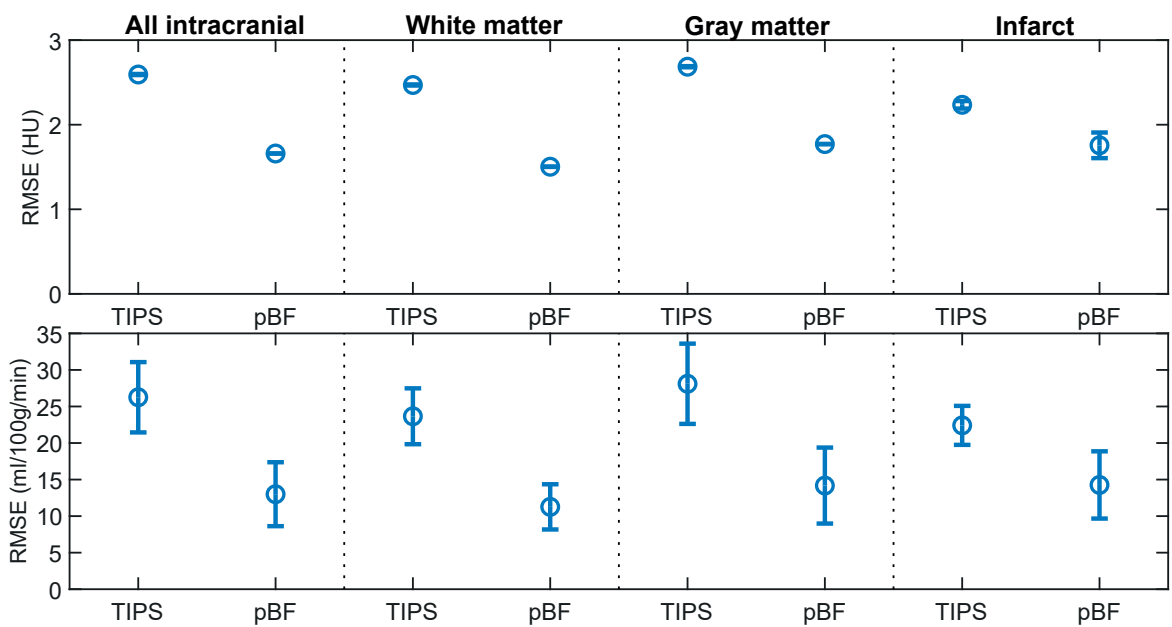

Figure 9.2 | The mean \pm standard deviation RMSE between filtered CTP scans and noise free CTP phantom for the different bilateral filters. The top row shows the error in the original CT numbers, and the bottom row shows the error in the cerebral blood flow (CBF). Abbreviations: TIPS; TIPS bilateral filter, pBF; PCA-guided bilateral filter.

The mean absolute CBF, CBV and MTT values of the phantoms are shown in Table 9.2. The pBF approximated the ground truth with the highest accuracy, except for the CBV values in the infarct and MTT values in the white matter where TIPS was significantly more accurate $(p<0.002)$. pBF estimated the CBF in the gray and white matter and CBV in the gray matter significantly more accurate than TIPS $(p<0.02)$. The other perfusion values did not show significant differences between bilateral filters.

Table 9.2 Mean absolute CBF (ml/100 g/min), CBV (ml/100 g) and MTT (s) values in the gray and white matter, and in the infarct core. Standard deviation within perfusion values are given between brackets. Most accurate mean perfusion values are highlighted in bold, and significant differences are marked with an asterisk.

\begin{tabular}{|c|c|c|c|}
\hline & GT & TIPS & $\mathrm{pBF}$ \\
\hline \multicolumn{4}{|l|}{ CBF } \\
\hline WM & $16.10(0.01)$ & $25.86(5.06)$ & $16.51(4.70)^{*}$ \\
\hline GM & $27.96(4.67)$ & $44.76(10.16)$ & $36.10(11.55)^{*}$ \\
\hline Infarct & $2.85(4.55)$ & $20.80(3.88)$ & $16.02(5.52)$ \\
\hline \multicolumn{4}{|l|}{ CBV } \\
\hline WM & $1.71(0.01)$ & $1.92(0.20)$ & $1.90(0.11)$ \\
\hline GM & $3.20(0.31)$ & $2.92(0.14)$ & $3.10(0.15)^{*}$ \\
\hline Infarct & $0.35(0.48)$ & $1.23(0.11)^{*}$ & $1.49(0.13)$ \\
\hline \multicolumn{4}{|l|}{ MTT } \\
\hline WM & $6.36(0.01)$ & $6.39(1.11)^{*}$ & $7.58(1.19)$ \\
\hline GM & 6.99 (0.99) & $5.31(1.16)$ & $6.03(1.10)$ \\
\hline Infarct & $21.91(10.53)$ & $6.32(1.03)$ & $6.68(1.55)$ \\
\hline
\end{tabular}

Abbreviations: GM; gray matter, WM; white matter, GT; ground truth, TIPS; TIPS bilateral filter, pBF; PCA-guided bilateral filter. 
Contrast-to-noise ratios are shown in Table 9.3. pBF CNR was higher for all perfusion maps compared to TIPS. GM-WM CNR for all perfusion maps was significantly higher for pBF ( $p<0.002)$. In addition, WM-infarct CBF-CNR, GM-infarct CBV-CNR and WM-infarct MTT-CNR was significantly higher for pBF compared to TIPS ( $p<0.007)$. Other CNRs were similar between TIPS and pBF.

Table 9.3 | Mean perfusion contrast-to-noise ratio (CNR) between gray and white matter, between gray matter and infarct core, and between white matter and infarct core. Standard deviation of the CNR is given between brackets. Highest mean CNR values are highlighted in bold, and significant differences are marked with an asterisk.

\begin{tabular}{llll}
\hline & GT & \multicolumn{1}{l}{ TIPS } & pBF \\
\hline CBF & & & \\
\hline GM-WM & 2.54 & $0.68(0.31)$ & $\mathbf{1 . 6 3 ( 0 . 5 6 ) *}$ \\
GM-infarct & 3.85 & $0.89(0.21)$ & $\mathbf{1 . 4 7 ( 0 . 8 9 )}$ \\
WM-infarct & 2.91 & $0.25(0.24)$ & $\mathbf{0 . 6 9 ( 0 . 3 9 ) ^ { * }}$ \\
\hline CBV & & & \\
\hline GM-WM & 4.82 & $1.24(0.33)$ & $\mathbf{3 . 1 5 ( 0 . 5 1 ) *}$ \\
GM-infarct & 4.97 & $1.98(0.24)$ & $\mathbf{3 . 5 3 ( 0 . 4 9 ) *}$ \\
WM-infarct & 2.81 & $0.85(0.20)$ & $\mathbf{1 . 1 9 ( 0 . 3 7 )}$ \\
\hline MTT & & & \\
\hline GM-WM & 0.64 & $0.31(0.22)$ & $\mathbf{0 . 8 5 ( 0 . 5 4 ) *}$ \\
GM-infarct & 1.41 & $0.16(0.11)$ & $\mathbf{0 . 6 5}(\mathbf{0 . 6 2})$ \\
WM-infarct & 1.48 & $0.16(0.12)$ & $\mathbf{0 . 7 8 ( 0 . 5 1 ) *}$ \\
\hline
\end{tabular}

Abbreviations: GM-WM; gray - white matter contrast-to-noise ratio (CNR), GM-infarct; gray matter - infarct CNR, WM-infarct; white matter - infarct CNR, GT; ground truth, TIPS; TIPS bilateral filter, pBF; PCA-guided bilateral filter.

In Figure 9.3, an example slice of CBF and CBV maps of the phantom are shown. The TIPS filter shows higher levels of noise in the perfusion maps, reducing contrast slightly compared to pBF. 


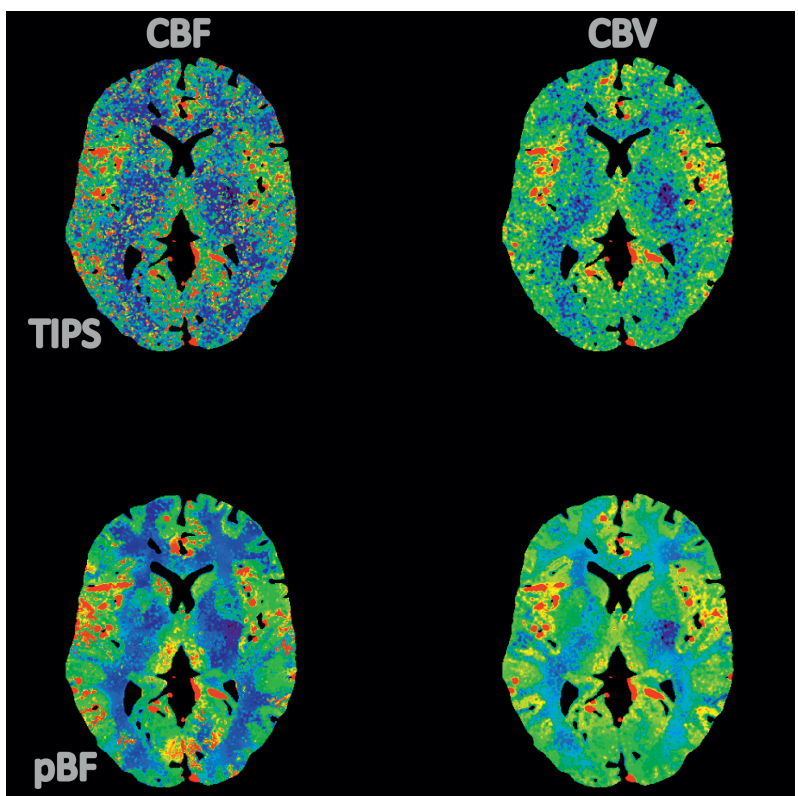

Figure 9.3 | Cerebral blood flow (CBF) (left column) and cerebral blood volume (CBV) (right column) maps of the phantom; in the left lentiform nucleus there is a small infarct visible (1.39 $\mathrm{ml}$ ). CBF ranges from $0-50$ and CBV from $0-5$. The TIPS filter shows higher levels of noise in the perfusion maps, reducing contrast slightly compared to pBF. Abbreviations: TIPS; TIPS bilateral filter, pBF; PCA-guided bilateral filter.

\section{Patients}

Sixty-eight consecutive patients were retrospectively identified; 10 patients did not meet inclusion criteria (thin-slice CTP unavailable or residual movement after registration). Fifty-eight consecutive patients were included (median age 68; IQR 54 - 77 years; $39.7 \%$ female), $37 / 58$ (63.8\%) of patients had signs of infarction on DWl; 30/37 (81.8\%) had a small-volume infarct $(\leq 4.2 \mathrm{ml})$. Baseline patient demographics are summarized in Table 9.4.

The mean patient perfusion values in the gray and white matter of TIPS and pBF1 are shown in Table 9.5. The CBF- and CBV-CNR is significantly lower for TIPS compared to $\operatorname{pBF}(p<0.001)$, while MTT-CNR is similar $(p=0.073)$. 
Table 9.4 | Patient demographics and baseline imaging characteristics.

\begin{tabular}{ll}
\hline Characteristics & Patients $(\mathrm{n}=58)$ \\
\hline Sex, male:female & $35: 23$ \\
Age (years), median (IQR) & $68.0(54.0-77.0)$ \\
Presentation NIHSS, median (IQR) & $2(0.8-4.8)$ \\
Cerebral infarct on DWI, $\mathrm{n}(\%)$ & $37(63.8)$ \\
$\quad$ Small-volume infarct $(\leq 4.2 \mathrm{ml}), \mathrm{n}(\%)$ & $30 / 37(81.8)$ \\
$\quad$ Large-volume infarct ( $>4.2 \mathrm{ml}), \mathrm{n}(\%)$ & $7 / 37(18.2)$ \\
Time symptom onset to CT (hours), median (IQR) & $2.2(1.2-4.2)$ \\
Time CT to MR (hours), median (IQR) & $26.4(19.3-39.6)$ \\
IV tPA administered, $\mathrm{n}(\%)$ & $15(25.9)$ \\
Thrombectomy, $\mathrm{n}(\%)$ & $5(8.6)$ \\
\hline
\end{tabular}

Abbreviations: IQR; interquartile range, NIHSS; National Institutes of Health Stroke Scale.

Table 9.5 | Mean absolute CBF (ml/100 g/min), CBV (ml/100 g) and MTT (s) values in the gray and white matter, and the gray - white matter CNR for TIPS and PBF. Standard deviation within perfusion values are given between brackets. Highest mean CNR values are highlighted in bold, and significant differences are marked with an asterisk.

\begin{tabular}{lll}
\hline & TIPS & pBF \\
\hline CBF & & \\
\hline WM & $15.64(4.31)$ & $13.46(5.38)$ \\
GM & $32.80(11.41)$ & $35.88(16.35)$ \\
GM-WM & $0.69(0.28)$ & $\mathbf{1 . 2 4}(\mathbf{0 . 5 2})^{*}$ \\
\hline CBV & & \\
\hline WM & $1.04(0.20)$ & $0.85(0.17)$ \\
GM & $1.86(0.44)$ & $2.04(0.44)$ \\
GM-WM & $0.80(0.36)$ & $\mathbf{1 . 5 7 ( 0 . 6 1 ) *}$ \\
\hline MTT & & \\
\hline WM & $8.88(2.66)$ & $6.20(2.54)$ \\
GM & $4.88(1.43)$ & $4.22(1.72)$ \\
GM-WM & $0.41(0.16)$ & $\mathbf{0 . 4 8}(\mathbf{0 . 3 3})$ \\
\hline
\end{tabular}

Abbreviations: GM; gray matter, WM; white matter, GM-WM; gray - white matter contrast-to-noise ratio, TIPS; TIPS bilateral filter, pBF; PCA-guided bilateral filter.

The sensitivity, specificity, PPV, NPV, accuracy, diagnostic certainty and image quality of infarct detection with thin-slice CTP is shown in Table 9.6. The sensitivity and specificity was not significantly different between filter techniques ( $p>0.060)$. NPV and accuracy was highest for PBF, and PPV was highest for TIPS. The 7 large-volume infarcts (infarct volume $>4.2 \mathrm{ml}$ ) were all detected by pBF, but TIPS missed one. 14 out of 37 patients with an infarct detected on DWI were not identified by any of the filter techniques. Diagnostic certainty was acceptable to good for all techniques, but did improve significantly with pBF compared to TIPS ( $p=0.022)$. Image quality also improved significantly from suboptimal/acceptable with TIPS to acceptable/good with $p B F(p<0.001)$. 
Table 9.6 | Sensitivity, specificity, positive and negative predictive value, accuracy (\%) given in mean with 95\% confidence interval and diagnostic certainty and image quality given in mean with standard deviation for TIPS and pBF. Highest mean values are highlighted in bold, and significant differences are marked with an asterisk.

\begin{tabular}{lll}
\hline & TIPS & pBF \\
\hline Sensitivity & $40.5(24.8-57.89)$ & $\mathbf{5 4 . 1}(\mathbf{3 6 . 9} \mathbf{- 7 0 . 5 )}$ \\
Specificity & $\mathbf{1 0 0 . 0 ( 8 3 . 9 - 1 0 0 . 0 )}$ & $90.5(69.6-98.8)$ \\
PPV & $\mathbf{1 0 0 . 0 ( 8 3 . 9 - 1 0 0 . 0 )}$ & $90.9(72.1-97.5)$ \\
NPV & $48.8(42.3-55.5)$ & $\mathbf{5 2 . 8}(\mathbf{4 3 . 4}-\mathbf{6 2 . 0})$ \\
Accuracy & $62.1(48.4-74.5)$ & $\mathbf{6 7 . 2}(\mathbf{5 3 . 7}-\mathbf{7 9 . 0})$ \\
Diagnostic certainty & $3.22(1.21)$ & \\
Image quality & $2.14(0.58)$ & $\mathbf{3 . 4 7}(\mathbf{1 . 0 5})^{*}$ \\
\hline
\end{tabular}

Abbreviations: PPV; positive predictive value, NPV; negative predictive value; TIPS; TIPS bilateral filter, pBF; PCA-guided bilateral filter.

Table 9.7 shows the results of the infarct location match. The total-ILM score of pBF was significantly higher than that of TIPS ( $p=0.008)$. For the individual anatomical regions, the difference in ILM was not significant between filter techniques ( $p>0.125)$. Dichotomization of ILM on infarct volume shows significantly higher total-ILM for small infarctions ( $\leq 4.2 \mathrm{ml}$ ) for pBF compared to TIPS ( $p=0.016)$. Small-volume infarct ILM in the anatomical regions was highest for pBF, but did not show any significant differences between filter techniques ( $p>0.250)$. There is no significant difference between techniques for large infarctions (>4.2 ml).

Table 9.7 | Infarct location match (\%) of TIPS and pBF with 95\% confidence interval are presented for all regions, cortical regions, basal ganglia and posterior circulation. Infarct size dichotomization is given for the different regions too. Highest mean values are highlighted in bold, and significant differences are marked with an asterisk.

\begin{tabular}{|c|c|c|c|c|}
\hline & Size $(\mathrm{ml})$ & DWI (n) & TIPS & $\mathrm{pBF}$ \\
\hline \multirow[t]{3}{*}{ Cortical } & All & 59 & $39.0(27.6-51.7)$ & $45.8(33.7-58.3)$ \\
\hline & $\leq 4.2$ & 49 & $32.7(21.2-46.6)$ & $38.8(26.4-52.8)$ \\
\hline & $>4.2$ & 10 & $70.0(39.7-89.2)$ & $80.0(49.0-94.3)$ \\
\hline \multirow[t]{3}{*}{ Basal ganglia } & All & 9 & $11.1(2.0-43.5)$ & $44.4(18.9-73.3)$ \\
\hline & $\leq 4.2$ & 9 & $11.1(2.0-43.5)$ & $44.4(18.9-73.3)$ \\
\hline & $>4.2$ & 0 & - & - \\
\hline \multirow[t]{3}{*}{ Posterior circulation } & All & 22 & $13.6(4.8-33.3)$ & $18.2(7.3-38.5)$ \\
\hline & $\leq 4.2$ & 22 & $13.6(4.8-33.3)$ & $18.2(7.3-38.5)$ \\
\hline & $>4.2$ & 0 & - & - \\
\hline \multirow[t]{3}{*}{ Total } & All & 90 & $30.0(21.5-40.1)$ & $38.9(29.5-49.2)^{*}$ \\
\hline & $\leq 4.2$ & 80 & $25.0(16.8-35.5)$ & $33.8(24.4-44.6) *$ \\
\hline & $>4.2$ & 10 & $70.0(39.7-89.2)$ & $80.0(49.0-94.3)$ \\
\hline
\end{tabular}

Abbreviations: cortical; cortical regions, TIPS; TIPS bilateral filter, pBF; PCA-guided bilateral filter.

Figure 9.4 shows a typical example of the thin-slice CTP with high noise levels at different time frames and the perfusion maps of a patient filtered with TIPS and pBF, with an infarction $(0.58 \mathrm{ml}$ on DWI) in the right superficial posterior cerebral artery. 


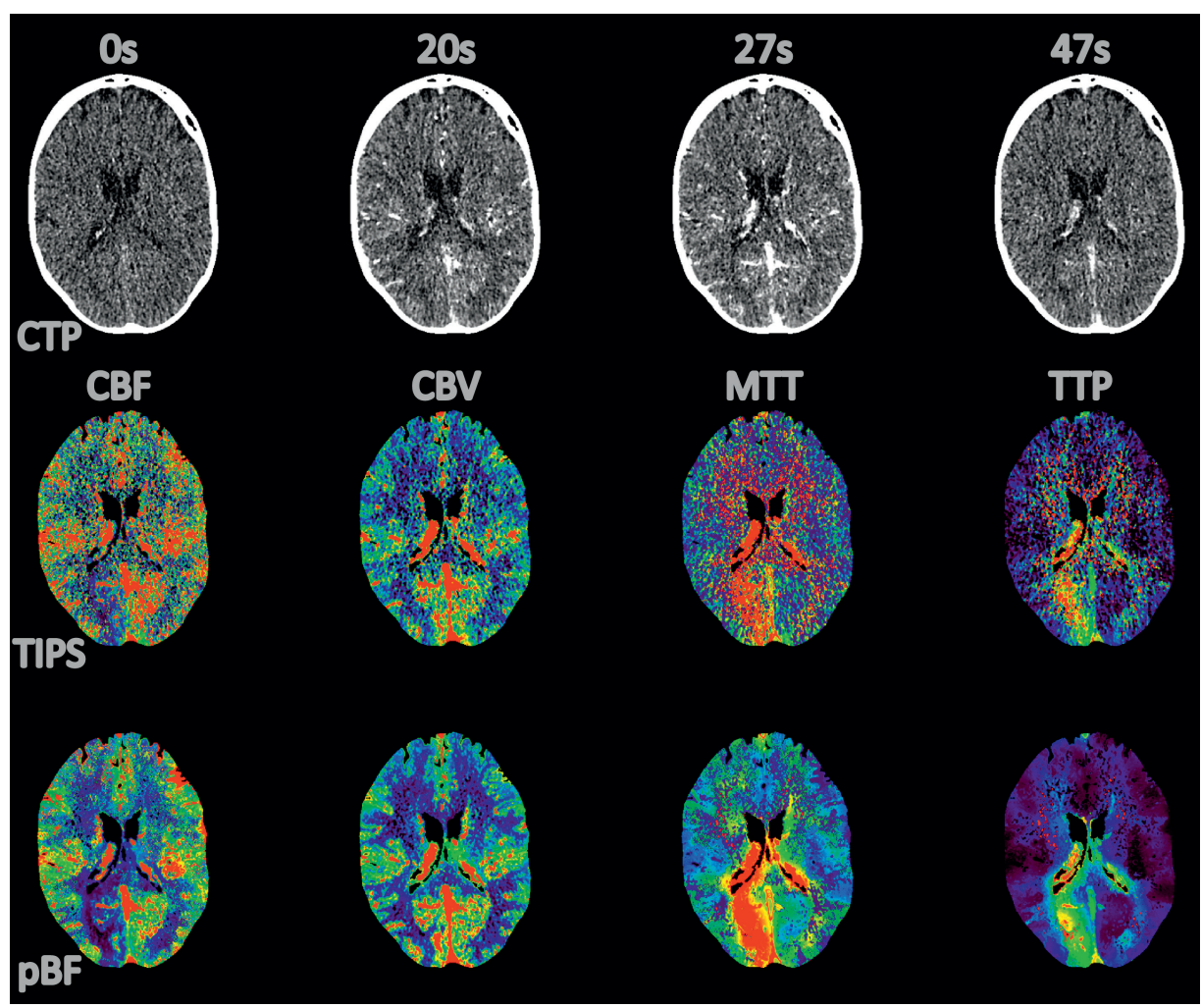

Figure 9.4 | CTP frames and perfusion maps of a patient with a right superficial posterior cerebral artery infarction, filtered with TIPS and PBF. The thin-slice CTP shows high levels of noise at all time frames. The affected region is most pronounced on the MTT and TTP map. The pBF shows high contrast and low noise on all maps and TIPS is substantially noisier. CBF ranges from 0 - 50, CBV 0 - 5, MTT 0 - 15 and TTP 0 - 10.

\section{Discussion}

CTP is clinically used to diagnose large vessel occlusions and to guide stroke therapy. Small-volume infarcts have clinical relevance in e.g. assessing probability for recurrent stroke, but are hard to detect with standard (thick-slice, usually 5 to $10 \mathrm{~mm}$ ) CTP protocols. One technical challenge in thin-slice CTP analysis is reducing the increased noise, which requires robust filtering. In this study we demonstrate that when using appropriate filtering, small-volume infarcts as small as $0.5 \mathrm{ml}$ can be detected, although sensitivity remains limited. In a comparison between two state-of-the-art filters (TIPS and $\mathrm{pBF}$ ) the accuracy for small-volume infarct detection improved from $62 \%$ with TIPS to $67 \%$ with pBF, but was still relatively low for small-volume infarct detection. The performance of TIPS on thin-slice CTP has been studied before, but was only evaluated for vessel visibility and ranked on image quality[151]. 
The TIPS noise filter, however, was not evaluated on perfusion values or its ability to detect small-volume infarcts. Whereas the TIPS filter measures the difference between (noisy) attenuation curves, pBF uses a guide image with a high contrast-to-noise ratio. In this way the filter kernel is shaped better to the underlying tissue curves and therefore allows for better preservation of the tissue curve.

Our phantom study provided an objective test to evaluate to what extend a bilateral filter is able to reconstruct the original noise free CTP acquisition. We have shown that guided bilateral filtering outperforms TIPS bilateral filtering. A significant difference was seen for CBF- and CT number-RMSE, for CBF and CBV perfusion values and most CNR measurements, which was similar to what we observed in the patient population. This, however, was opposite of what was seen in prior research, in which an averaged-timeframe guided bilateral filter and TIPS were compared on thick-slice reconstructions[149]. Taking all noisy time frames into account, the sum of squared differences measure used in TIPS is very susceptible to noise; it acts on 100\% of the information and $100 \%$ of the noise. The first PCA component, however, contains about $70 \%$ of the information at just 5\% of the noise (in case of 20 time-frames). Since thinslice CTP has much more noise, TIPS is affected substantially more than guided bilateral filtering explaining the significant improvement seen with $\mathrm{pBF}$.

In the patient population, CNR was generally significantly higher for pBF compared to TIPS. The CBF map is important for the identification of infarction[156,157]; using the pBF we saw a significant increase in CBF- and CBV-CNR, which resulted in the nonsignificant trend towards improved infarct sensitivity using guided bilateral filtering. The results, however, also show that the bilateral filters presented here were not sufficiently able to identify small-volume infarctions; which resulted in a relatively low sensitivity and negative predictive value for both filtering techniques. The proposed pBF, however, has shown highest sensitivity, and high specificity for infarct detection. The diagnostic certainty and image quality increased significantly.

The ability of the bilateral filters to localize infarcts was assessed using ILM for predefined cerebral regions. Total-ILM of pBF showed a significant improvement in locating infarcts compared to TIPS (38.9\% vs TIPS: 30.0\%), especially for the small-volume infarctions (33.8\% vs TIPS: 25.0\%). This reflects the higher CNR values as seen in the phantom and patients, which increases conspicuity of small-volume infarctions on thin-slice CTP. In our study, we saw a trend towards an increase in ILM for the cortical regions and basal ganglia using pBF compared to TIPS. Verification of trend requires testing in a larger cohort of small-volume infarct patients. ILM analysis also showed that large infarctions (>4.2 $\mathrm{ml}$ ) are detected equally well with the different filters. 
The fact that 14 out of the 30 small infarctions were not detected by any of the filters illustrates that such infarctions remain hard to detect, even on thin-slice CTP. Analysis of infarct volumes on DWI showed that for 9 out of the 14 patients with infarctions missed by both filters, infarcts were less than $0.75 \mathrm{ml}$ in size. A similar observation was made for the basal ganglia: 4 out of 9 infarctions were smaller than $0.25 \mathrm{ml}$ and not detected by any of the techniques. This shows that the infarctions we were trying to detect were extremely small. The ILM analysis also showed that infarct localization for small-volume infarctions is especially difficult in the posterior circulation. Only 2 out of 17 small-volume infarctions located in the cerebellum, pons and midbrain were detected. These areas are susceptible to severe bone artifacts and photon starvation, which usually results in low tissue attenuation signal. This reduced signal affects the perfusion analysis substantially, introducing artifacts and noise in the perfusion maps, and making detection of small-volume infarctions very difficult. Lastly, for 3 out of 14 patients (part of) the infarction was not in the field-of-view of the CTP. These infarcts were all located in the top of the cranium; an area which is not only difficult to image due to imaging artifacts, but also frequently missed due to insufficient axial coverage. This illustrates that full brain coverage is essential for thin-slice CTP.

The limiting factors for thin-slice CTP currently thus are contrast, artifacts and noise, and early infarcts might not even show reduced CBV or prolonged MTT. This means that in order to increase sensitivity for small-volume infarct detection a number of steps need to be taken. First of all, the field-of-view of the CTP needs to be increased so that the entire brain and all infarction are in the image. Second, contrast needs to be increased and artifacts need to be reduced. A possible solution for the latter might be dual-energy $C T$, which is known to reduce artifacts in the posterior fossa[23,158]. To increase contrast, it might pay out to increase the amount of contrast agent, as long as this outweighs the possible risks of higher contrast agent dosage.

The presented study has a number of limitations, including the retrospective design of the study. We also could not compare absolute infarct core volumes to follow-up imaging, since we do not have a validated approach or thresholds for infarct volume estimation for thin-slice CTP analysis. Furthermore, time is of the essence in stroke imaging and post-processing. Currently, the presented guided bilateral filter is not fast enough for clinical use (taking well over half an hour to filter a scan with 99 slices, a 512x512 matrix and 30 time frames). The TIPS filter, however, is substantially slower than the guided bilateral filter. In addition, in at least three patients the axial coverage of the CT perfusion scan did not prove sufficient to cover the entire brain. This resulted in a miss of infarction on CTP. This shows that for thin-slice CTP to approach the accuracy of DWI full brain coverage is a requirement. Lastly, CTP scans were acquired on two different CT scanners. Both scanners have their own optimized CTP protocol, which might have a minor influence on our results. The current dataset, however, is too small to test this hypothesis. 
Part III | CT Perfusion in stroke imaging

In conclusion, this study showed there is value in thin-slice CTP in the detection of small-volume infarcts. Perfusion values are estimated more accurately and with higher contrast using guided bilateral filtering compared to TIPS. While the detection of smallvolume infarctions remains difficult, infarcts could be detected with higher sensitivity and significantly higher diagnostic certainty and improved image quality using guided bilateral filtering than with the current state-of-the-art TIPS filter. 
Thin-slice CT perfusion for small-volume infarction | Chapter 9 



\section{CHAPTER 10}

\section{SUMMARY AND DISCUSSION}

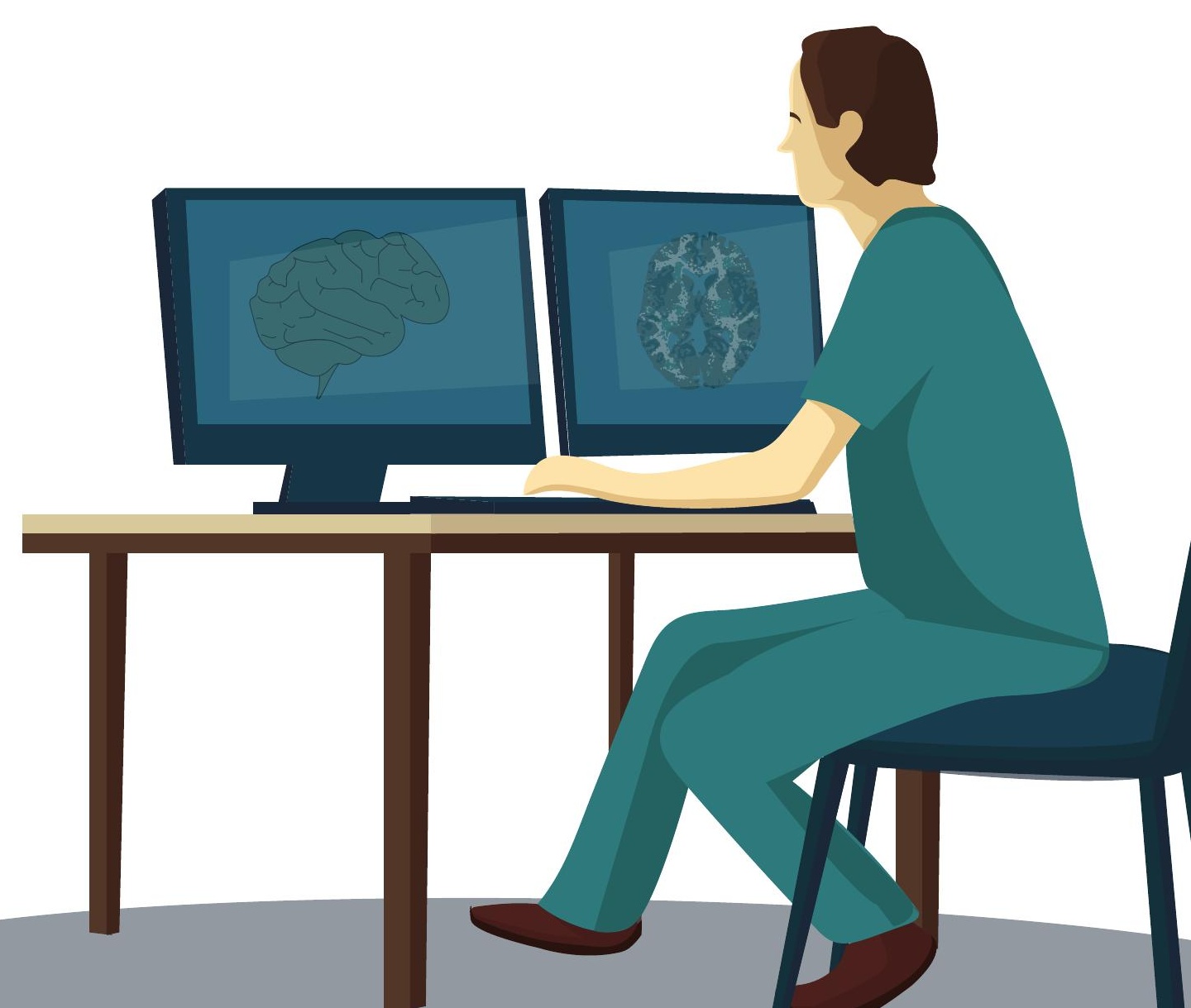




\section{Summary}

$\mathrm{CT}$ is often used as the modality of choice for the diagnosis of patients with suspicion of stroke. CT is a fast imaging modality, and is therefore preferred inasmuch as a stroke requires quick diagnosis and treatment after first symptom onset. CT imaging allows visualization of occluded blood vessels, but also identification of location and volume of the infarction. The volume of the infarction is an important guidance for the treatment of the patient. Current CT technological innovations are aimed at increasing the diagnostic image quality, increasing the predictive value and quantitative accuracy of $C T$, and reducing the radiation dose. A number of these issues can be achieved with so called dual-energy CT (DECT), in which two CT images are acquired at different photon energies. Part / of this thesis is focused on evaluation of the technical aspects of dual-energy CT in comparison with conventional CT.

In Chapter 2, the radiation dose over a broad range of clinical protocols was evaluated using a dual-layer detector CT scanner and a technically equivalent conventional singlelayer detector CT scanner, to investigate whether a dual-layer detector increases dose levels. We showed that dual-layer detector CT, using a $120 \mathrm{kVp}$ tube potential, can be routinely used in daily clinical practice to provide additional information without increasing radiation dose when compared with a conventional single-layer detector CT scanner.

In Chapter 3, the image quality of conventional CT images acquired with a dual-layer CT scanner was compared with that of images acquired at a conventional single-layer CT scanner, at equivalent dose levels using phantoms. Just as the radiation dose, image quality might be affected by the introduction of the dual-layer detector. We found that the image quality of conventional CT images acquired on a dual-layer CT scanner is similar to its counterpart on a conventional CT scanner for medium-sized phantoms, and slightly lower for (very) large phantoms at lower tube voltages. Accordingly, we concluded that the introduction of a dual-layer detector, with the advantage of having dual-energy or spectral information, neither compromises image quality of conventional images nor increases radiation dose for normal-sized patients, and only slightly degrades image quality for large patients.

In Chapter 4, the clinical image quality of dual-energy non-contrast brain $\mathrm{CT}$ virtual monochromatic images (VMI) acquired on a dual-source CT scanner was assessed and compared with conventional CT. We found that non-contrast DECT VMI significantly improves the image quality of non-contrast brain CTS compared with conventional CT. The optimal VMI energy was found to be dependent on the clinical indication of the acquisition: reconstructions with a special DECT beam-hardening correcting kernel at $100 \mathrm{keV}$ are preferred for scans close to the skull or in the infratentorial area to minimize artifacts. For supratentorial diagnostics, in which high SNR and CNR are more important, reconstruction with a standard head kernel at 65 keV are preferred. 
Part // focusses on the added value of dual-energy CT in stroke imaging in comparison with conventional CT imaging.

In Chapter 5, the potential to diagnose early infarcts using non-contrast brain DECT VMI, reconstructed with a special DECT kernel, was evaluated. In Chapter 4 it was demonstrated that DECT VMI improved contrast-to-noise profiles and reduced beam hardening artifacts relative to conventional $\mathrm{CT}$, but the use of $\mathrm{VMI}$ in the detection of cerebral infarcts has not been established. We found that non-contrast head VMI at 80 - 90 keV more accurately detected and localized infarcts compared with conventional CT, which was in agreement with the signal-to-noise improvements with the DECT kernel seen in Chapter 4.

In Chapter 6, the potential of virtual ischemia maps of non-contrast DECT in patients with acute ischemic stroke was evaluated. A virtual ischemia map is generated by using a standard three-material decomposition originally aimed at separating hemorrhagic tissue (blood), water, and iodine. Since iodine is not present in non-contrast CT, the algorithm acts as a two-material separation algorithm and consequently highlights areas which have altered water content. This reduces gray-white matter contrast, but tends to preserve edema and increase conspicuity. We found that virtual ischemia maps were more accurate than conventional CT in approximating infarct core volumes and the infarct location. This suggests that DECT more accurately differentiates between infarcted and healthy tissue than conventional CT.

In Chapter 7, the added value of dual-energy VMI CT perfusion scans to the quality of the perfusion maps was investigated. Low keV VMI specifically selects data closer to the $33 \mathrm{keV}$ iodine k-edge as compared with conventional CT, thus potentially increasing the iodine contrast of CTP acquisitions. The generation of the low keV virtual monoenergetic CT images, however, comes at the cost of increased noise levels compared with conventional CTP. We found that the image quality and visual quality of $50 \mathrm{keV}$ CT perfusion maps is superior to that of conventional 80 and $120 \mathrm{kVp}$ images.

In part I/I we evaluated the technical aspects of CT perfusion in CT stroke imaging.

In Chapter 8, the influence of the acquisition interval on three different CTP analyses is investigated by evaluating quantitative perfusion maps and infarct volumes. It is hypothesized that shorter acquisition intervals would allow more precise monitoring of the contrast passage resulting in more accurate estimations of the perfusion parameters. However, dose and technical restrictions (such as jog techniques to increase the scan length) often limit the shortest achievable acquisition interval. 
We found that increasing the acquisition interval may introduce a bias in the perfusion parameters, but this bias can be corrected by calibration of the perfusion maps and therefore still allow distinction between healthy and infarcted tissue. Infarct volumes can likewise be influenced by the acquisition interval, but visual inspection indicated minor differences in infarct volumes between acquisition intervals. For a commercial block-circulant singular value decomposition (bSVD) perfusion analysis package acquisition intervals up to 4 seconds could be achieved, and for a non-commercial bSVD and a non-linear regression (NLR) model-based method intervals up to 5 seconds.

In Chapter 9, the ability to detect small-volume infarctions (i.e. smaller than $4.2 \mathrm{ml}$ ) with thin-slice CTP by noise reduction using two bilateral filters is evaluated. Small-volume infarctions and posterior infarcts are clinically relevant but hard to detect with current CTP protocols. It has been suggested that thin-slice CTP may improve the detectability of small-volume infarcts, but will require more sophisticated image filters to reduce inherently increased noise. The evaluated bilateral filters were the time-intensity profile similarity bilateral filter (TIPS) and a novel bilateral filter guided by a principal component image. We found that perfusion values are estimated more accurately and with higher contrast using guided bilateral filtering compared with TIPS filtering on thin-slice CTP. While the detection of small-volume infarctions remains difficult, infarcts could be detected with higher sensitivity and significantly higher diagnostic certainty and improved image quality using guided bilateral filtering than with the current stateof-the-art TIPS filter. 


\section{General Discussion}

This thesis was focused on the possibilities of dual-energy CT (DECT) to improve the diagnostic performance for ischemia detection and the use of DECT and high-resolution thin-slice CT perfusion to improve CT perfusion analysis.

\section{Dual-energy CT}

Dual-energy CT has proven its potential in neuroimaging by metal and bone artifact reduction[159,160], differentiation of iodine extravasation from hemorrhage[25,69,97] and the evaluation of vascular stenosis and aneurysms[161-164].

The research in this thesis has demonstrated the additional potential of DECT for the evaluation of brain parenchyma in (suspected) stroke patients. The presented studies found that by using non-contrast DECT VMI the sensitivity and specificity for the detection of acute infarction in suspected ischemic stroke patients is improved compared with conventional CT, especially in the early time window of $\leq 4.5$ hours after last-seen-well. In addition, non-contrast DECT virtual ischemia maps may be used to more accurately estimate core infarct size in large-vessel occlusion patients, again especially in the $\leq 6$ hours after last-seen-well time window. This means that non-contrast DECT images do not only improve infarct detection, but also improve delineation of the infarction on non-contrast CT compared with conventional CT. Given that patients are eligible for intravenous thrombolytic therapy until $\leq 4.5$ hours after last-seen-well, and that large-vessel occlusion triage is highly reliant on accurate neuroimaging to identify treatment candidates[10,119], we expect that, for patients imaged in the early-window, clinical certainty can be improved with the use of DECT. Moreover, we expect that DECT non-contrast CT will be a valuable improvement of the CT stroke protocol for the initial assessment of patients with suspicion of stroke in general. We do, however, not expect that NCCT DECT reconstructions will replace CT perfusion for infarct volume estimations, but NCCT DECT may assist CTP in establishing infarcted tissue.

It should be noted that both $\mathrm{VMI}$ and virtual ischemia maps are reconstructed from the same DECT data. This means that in essence the same information is used to visualize infarction. VMI is an energy-dependent weighted image of the photoelectric effect and the Compton scatter components at that energy level. A virtual ischemia map is a three-material decomposition in which hemorrhage, water, and iodine volume fractions are determined for each voxel (essentially a virtual non-contrast reconstruction). Since iodine content is zero, ischemia maps essentially only contain two materials and therefore separate altered water content (infarction). This means that even though the source data for the reconstructions is the same, $\mathrm{VMI}$ and ischemia maps are different methods to process the DECT data resulting in different ways to visualize ischemia. 
Since both DECT reconstructions come as an addition to conventional $C T$, it means that with a single DECT scan conventional CT, VMI and ischemia maps are available, which might increase diagnostic certainty for the neuroradiologists. However, both non-contrast VMI and virtual ischemia maps require further evaluation in larger prospective studies to investigate the effectiveness of these DECT images in clinical decision making. Since virtual monochromatic images and virtual ischemia maps can readily be reconstructed with vendor-provided software, translation of the VMI and virtual ischemia reconstructions to the clinical workflow is straightforward. It should be mentioned, however, that diffusion-weighted MR imaging is still considered the gold standard for the detection of infarction in acute ischemic stroke and that CT imaging has not (yet) reached this level of accuracy[86].

In neuroimaging, the use of DECT VMI can improve image quality in comparison with conventional CT. However, we showed that the image quality is highly dependent on the used reconstruction kernel, which should therefore be chosen carefully. The main aspect to consider for the reconstruction kernel is the part of the brain that is going to be imaged. In clinical practice, this probably requires multiple reconstructions per patient. While this could increase diagnostic certainty, adding more images for the evaluation of a patient may compromise the workload of radiologists. A possible solution to this problem is adaptive reconstruction kernels. An adaptive reconstruction kernel is a kernel that is adapted to its environment, which means that it is shaped like a beam-hardening correcting kernel close to the skull and in the infratentorial area to reduce artifacts, and more like a standard head kernel in the supratentorial region to optimize gray-white matter contrast. This means that the reconstruction is optimized for multiple indications and does not increase the number of images that need to be reviewed by the radiologist.

Chapters 2 and 3 showed that a DECT CT scanner can be implemented in clinical routine without increasing radiation dose or loss of image quality. Until now, DECT application was hampered by the requirement of specific scan protocols. The introduction of the dual-layer CT scanner allowed retrospective DECT reconstructions on every recorded CT acquisition at high (i.e. 120 and 140 kVp) tube voltage. However, a disadvantage of dual-layer CT is that it is not possible to have a DECT acquisition at $80 \mathrm{kVp}$, which means that for some examinations the tube voltage needs to be increased from 80/100 kVp to $120 \mathrm{kVp}$ if the use of DECT is indicated. The higher kVp might have an influence on the image quality and radiation dose levels of those acquisitions, which calls for further investigation. In Chapter 2, however, we saw that in 6 out of 15 clinical protocols the tube voltage had to be increased from 80/100 kVp to $120 \mathrm{kVp}$, but this did not result in a significant increase in dose or a decrease in diagnostic image quality. In addition, Chapter 7 has shown that changing the CTP acquisition from 80 to $120 \mathrm{kVp}$ does not result in an increase in radiation dose, and with DECT virtual monoenergetic images can improve image quality. 


\section{CT perfusion}

CT perfusion has proven a valuable selection tool for endovascular therapy in recent years[99,108]. For CTP to be used as a selection tool it needs to be reliable and allow quantitative core-penumbra volume mismatch evaluation. In this thesis, we have shown the reliability of CT perfusion with longer acquisition intervals. Longer acquisition intervals in CTP are seen in CT scanners which use Jog Mode or helical shuttle mode scanning to extend the axial coverage in order to include the whole brain. The perfusion values and visual infarct volume maps of CTP with acquisition intervals up to 5 seconds were found to provide reliable results, which means that we have shown that extending the axial coverage to full brain coverage is possible. Increasing the brain coverage decreases the chance on missing an infarct substantially.

We have also shown that with DECT CT perfusion imaging the quality of perfusion maps can be increased significantly when using $50 \mathrm{keV}$ VMI DECT reconstructions instead of conventional CT reconstructions. This improvement can be used to estimate infarct volumes more reliably and it increases diagnostic confidence for neuroradiologists without sacrificing image quality or increasing ionizing radiation dose. This means that DECT CTP imaging has great potential for reliable and quantitative infarct volume estimation. However, this needs to be confirmed in a larger and prospective cohort.

We found different optimal VMI energies for CTP (50 keV) and NCCT (80 - $90 \mathrm{keV}$ ), because in a CTP acquisition iodine contrast agent is used. lodine has a k-edge of 33.2 $\mathrm{keV}$, which means that there is an increased attenuation at lower tube voltage energies, i.e. there is more signal at lower energies. In NCCT iodine contrast is not present and therefore a higher tube voltage energy is needed to maximize image contrast, which translates into a difference in optimal VMI energy between NCCT and CTP. This is also the reason why conventional CTP is usually acquired at $80 \mathrm{kVp}$ and NCCT at $120 \mathrm{kVp}$.

We have seen in numerous cases that thick-slice CTP is good for detection and reliable size estimation of large infarcts[165-167], but that small-volume infarcts (2 - $20 \mathrm{~mm}$ in diameter; $\leq 4.2 \mathrm{ml}[144]$ ) are very hard to identify with current CT perfusion acquisitions and analyses. We have shown that guided bilateral filtering, in combination with nonlinear model-based CT perfusion analysis, is able to increase the accuracy of smallvolume infarct detection as compared with TIPS bilateral filtering. Chapter 9 also showed that a large number of small-volume infarctions are not detected. The limiting factors for thin-slice CTP currently are contrast, imaging artifacts, noise, and the fact that early infarcts might not even show reduced CBV or prolonged MTT. The latter is something that needs further investigating, but in order to increase the sensitivity for small-volume infarct detection a number of improvements are required. We have shown that using a high-contrast PCA-guided bilateral filter the noise levels of a CTP acquisition can be suppressed. There might, however, be more suitable options for this task. 
To increase contrast, it might pay out to increase the amount of contrast agent, as long as this outweighs the possible risks of higher contrast agent dosage. Another option would be to try and update the non-linear model-based non-linear regression deconvolution (NLR) CTP analysis. Currently, model-based CTP analysis does not require any a priori knowledge. This means that the model fits the signal to the best of its ability. We know, however, that in an infarct the signal by definition is low which makes model fitting difficult. This results in large uncertainties in parameters, which adds noise to the perfusion maps and thus makes small-volume infarct detection a challenge. By adding a priori knowledge about the underlying perfusion mechanisms, perfusion modelling may be able to handle low signal infarct pixels with a higher accuracy and thereby allow better detection. Lastly, additional research needs to identify approaches most suited for reliable calculation of small infarct volumes based on the perfusion maps. Current infarct volume calculations rely on relative and absolute thresholds which are validated on thick-slice CTP perfusion maps and need to be optimized for thin-slice CTP. These infarct volumes are of importance in therapy decision, and therefore investigation of this issue is a priority.

\section{Limitations}

There are some limitations to the studies presented in this thesis. First, we have investigated a number of different applications of DECT in the acute stroke setting. Every investigation was conducted using a single method of acquiring a DECT dataset: either dual-layer (Chapters 2, 3 and 7) or dual-source CT (Chapters 4, 5 and 6). DECT acquisitions and reconstruction algorithms vary between manufacturers and types of DECT scanners. Consequently, our results may not simply be transferred between duallayer, dual-source or rapid kV switching CT scanners[79]. Prior studies have shown that there are differences in iodine quantification and monochromatic image attenuation between DECT scanner platforms[168,169]. This means that the DECT results shown in this thesis need to be verified for every application of every vendor separately.

Second, DECT scanners are relatively expensive and produce large DECT datasets. This could pose a problem for smaller institutes, or institutes with low funds. The question is whether or not the additional costs associated with a DECT scanner outweigh the benefits of DECT, which is still subject to research for a variety of applications. For stroke imaging, a DECT scanner has proven its potential and would strengthen diagnostics.

Third, the CTP studies in this thesis lack a validated approach to estimate infarct volumes using the non-linear model-based non-linear regression deconvolution (NLR) method. This means that absolute infarct volumes are not available and could not be compared with follow-up diffusion-weighted imaging even though the infarct volumes are an important measure in CTP imaging. This should be part of future investigations. 
Fourth, as mentioned earlier, in stroke imaging the term "time is brain" is of importance, and therefore the results of a CTP analysis need to be available to the radiologists within a few minutes. For now, the proposed filter techniques in this thesis do not meet that requirement (with processing times well over 30 minutes) and are therefore clinically not acceptable yet.

Finally, the CT scanners used in the current studies all have a maximum of $8 \mathrm{~cm}$ axial coverage (Jog Mode or $8 \mathrm{~cm}$ axial detector) for the CTP acquisition. This means that full brain coverage sometimes is not possible and can lead to false negatives for infarct detection. Consequently, full brain coverage is a requirement for CTP to approach the accuracy of DWI.

\section{Future perspectives}

One of the limitations mentioned is the speed of CTP image processing with the methods presented in this thesis. Advances in computational speed in CPU and GPU, but also new methods to accelerate bilateral filtering, which is an active field of research[170,171], can decrease processing times and make thin-slice perfusion scans using model-based CTP analysis and bilateral filtering clinically available. Image processing times might also be reduced using machine learning. Noise reduction using the guided bilateral filter proposed here may be used as the input dataset to train a network for CTP filtering.

As mentioned in the introduction of this thesis, the major drawback of CT imaging is the use of ionizing radiation. In recent years, use of automatic tube voltage or exposure control and use of iterative reconstruction have contributed to decreasing dose levels to the patient[172-174]. A promising option for reducing dose in stroke imaging is the use of more elaborate iterative reconstruction. Currently thin-slice CTP acquisitions at our institution are reconstructed with statistical (hybrid) iterative reconstruction $\left(\right.$ iDose $\left.^{4}\right)$, whereas it has been shown that model-based iterative reconstructions (MBIR) can reduce dose significantly $[64,175,176]$. This could contribute to a substantial dose reduction, but caution needs to be taken since it has been reported that there can be changes in image texture[177] and sometimes image artifacts that can affect the diagnosis and acquisition[175]. For stroke imaging the main limiting factor now with MBIR is the computational burden, i.e. reconstruction times are too high and therefore not realistic for thin-slice CTP at this time. Another method to reduce radiation dose associated with the CT stroke protocol is to replace CTP with so called multiphase CTA (mCTA). mCTA is a technique in which at three time points a CTA is acquired to assess collateral circulation[178]. This assessment is then used for treatment decision making[179,180]. 
There are, however, a number of problems associated with mCTA. MCTA does not allow calculation of core-penumbra volume mismatch which is an important parameter in the treatment decision and it is difficult to precisely time the three acquisition time-points in conjunction with the contrast concentration passage through the vasculature[181]. This last problem has led to the introduction of dynamic CTA (dCTA), in which more time points (usually $>15$ ) are used to allow complete tracking of the contrast bolus[181]. This approach is comparable to the dose reduction for CTP imaging as proposed in Chapter 8 , in which we increased the acquisition interval and still obtained reliable CTP results at a reduced dose. This approach seems preferable to dCTA, although a direct comparison still has to be made.

The fact that CTP is fast, low in cost and has few contraindications has made it an essential part of early assessment of suspected ischemic stroke patients[182-184], even though diffusion-weighted MRI does not involve radiation dose, whereas CTP does. The current acute stroke protocol in our institute has an effective dose of less than $10 \mathrm{mSv}$ for the patient, which is 3 to 4 times higher than what humans experience annually by natural radiation, but well below the 100 mSv a year at which an increase in cancer risk is clearly evident[183] (albeit still small). There are, however, large differences in radiation dose levels between institutes[183,185], which originates from differences in acquisition parameters and differences between vendors[103,186]. Moreover, differences in image processing packages result in significantly different perfusion maps, even for identical CTP acquisitions $[16,186]$. The differences in dose due to differences in acquisition parameters and vendors, and differences in image processing show that efforts need to be made to standardize and optimize CTP imaging acquisition, dose efficiency and image processing, or to develop a way to normalize CTP data between vendors and institutions. This will require more cooperation between vendors, but also more insight in the different algorithms so that CTP results are independent of scanner type or processing software. Currently, a joint effort of 17 Dutch stroke intervention centers is investigating the possibilities of CTP harmonization[187]. Standardization of CTP will make it a definite mainstay in the evaluation of acute stroke patients, and will increase quantitative accuracy.

Detection of small infarctions remains difficult, especially in the posterior circulation (i.e. cerebellum, midbrain and pons). In Figure 10.1, a patient from Chapter 9 with an infarction in the right cerebellum is shown. Owing to a large beam hardening artifact in the pons (mainly visible on the MTT and TTP maps) the infarct in the cerebellum is missed. The perfusion maps do not give enough diagnostic certainty for the radiologist to be able to discriminate between the artifact and the infarction. A possible solution for reducing these beam hardening artifacts is the use of DECT. In Chapter 4, we have shown that the posterior fossa artifact index is reduced by using $100 \mathrm{keV} \mathrm{VMI}$ images compared with conventional CT imaging. This result could also be applied to CT perfusion imaging; possibly reducing beam artifacts and with that increasing diagnostic certainty for the radiologist. 


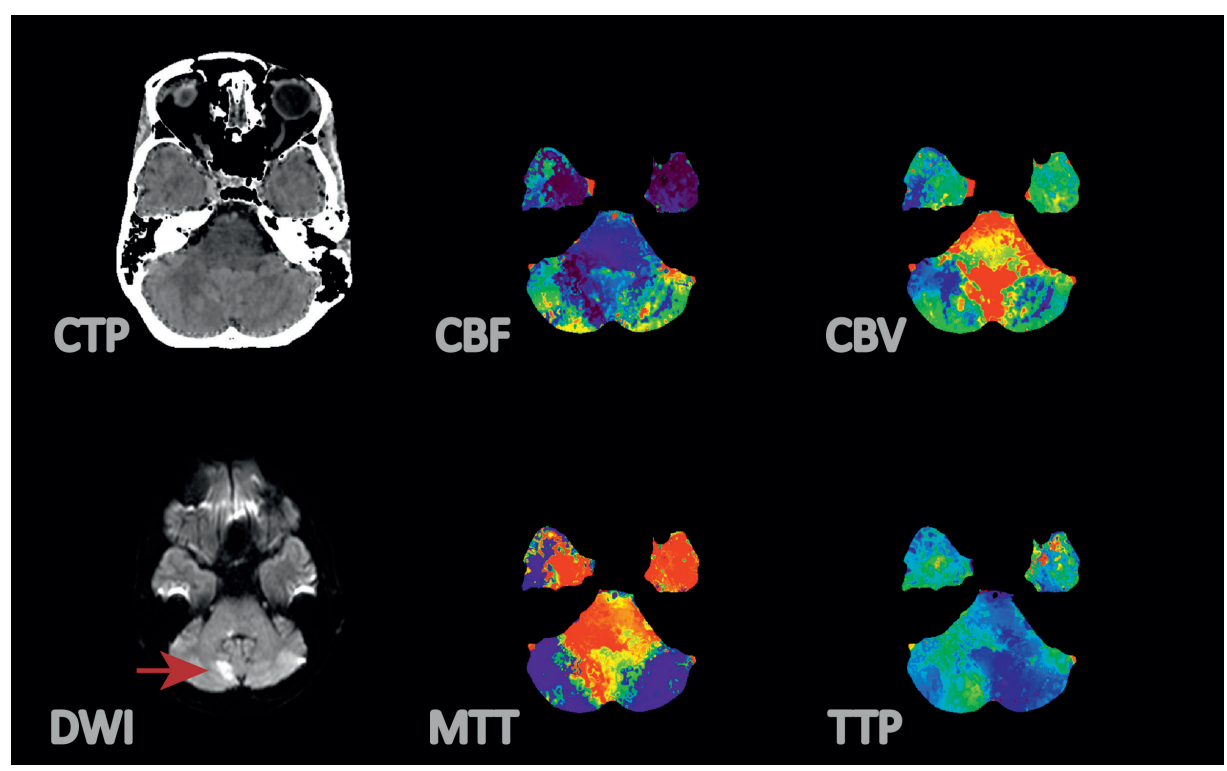

Figure 10.1 | Perfusion maps of a patient with an infarction in the right cerebellum (indicated with arrow on DWI), which was undetected by thin-slice CTP owing to a beam hardening artifact in the pons. The artifact results in overestimated MTT values, making discrimination between artifact and infarction very difficult.

DECT offered a variety of new applications, which can be used to increase diagnostic performance of CT in the acute stroke setting as we have shown in this thesis. However, there are other possibilities to implement DECT in neuroimaging, and especially in acute stroke imaging. We have shown that with vendor-provided DECT reconstructions infarct detection and delineation can be improved. It might, however, be possible to create new dedicated reconstructions using material decomposition, similar to edema maps[89,91,188], to improve visualization of infarctions. Furthermore, the PCA-guided bilateral filter presented in this thesis could be altered for use in a DECT CTP acquisition with the low- and high-energy information as input for the principal component analysis. In this way the amount of information is increased for the PCA components, which might improve contrast in the PCA-guided image and thereby reduce noise in the CT perfusion image. Another possibility would be to add additional guide images to the guided bilateral filter. These guide images could be generated with DECT derivatives such as virtual monoenergetic CT images. A low keV maximum intensity projection guide image could be used to identify vasculature, and a high keV (and thus low noise) mean guide image to identify brain structures. This approach would allow the two DECT-generated guide images to improve noise reduction. 
In this thesis we mainly focused on identifying infarction, but DECT could also be used to locate and characterize major causes of infarction. There have been previous studies showing the potential of DECT to detect thrombi in the heart[189] and characterize large artery atherosclerosis[190-192], which are considered primary causes of stroke. By scanning the heart, neck, and brain it becomes possible to detect these sources. The potential of scanning heart, neck, and brain in a single acute stroke evaluation has been shown by our group before, which also demonstrated the improved visualization of intracardiac thrombus using DECT[193] (Figure 10.2). The detection and characterization of the stroke sources can be used as risk stratification of patients to identify plaques that are vulnerable or thrombi that are about to break from the vessel wall. These at-risk patients could then receive treatment before a (recurrent) stroke occurs.

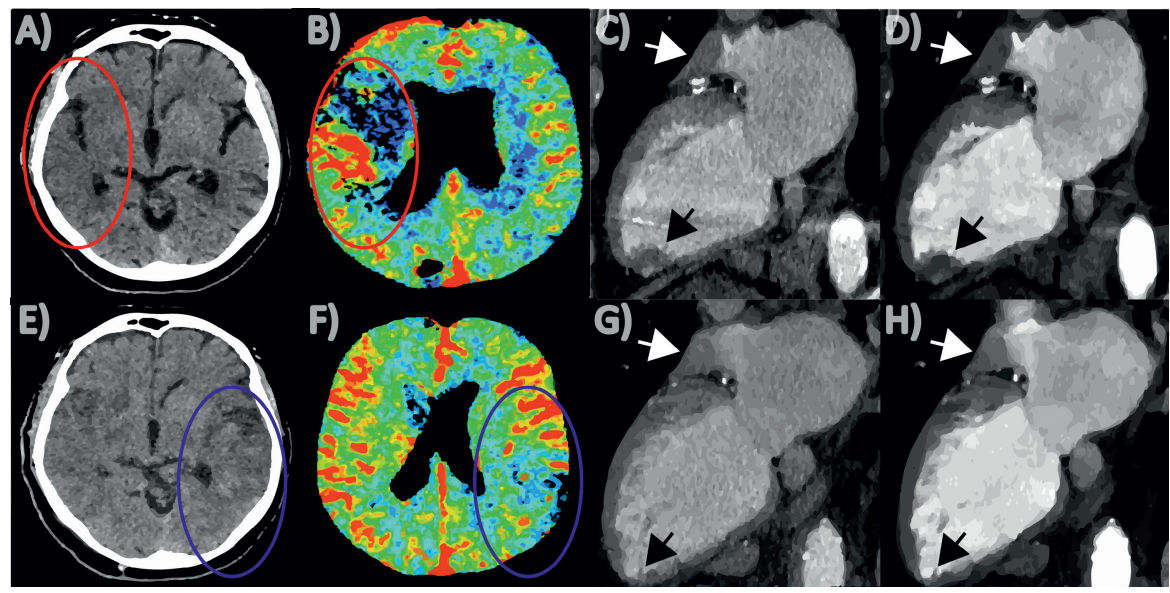

Figure 10.2 | Example of acute stroke evaluation covering the base of the heart up to the crown of the head. The top row is the patient at admission; the bottom row is the same patient with follow-up imaging after a second ischemic event. Shown are A) non-contrast CT with early signs of ischemic stroke (red oval) and B) CTP showing perfusion deficit in the territory of the right middle cerebral artery (red oval). Frames C) conventional and D) $40 \mathrm{keV}$ monoenergetic admission (TA show two thrombi, one in the left atrial appendage (white arrow) and one in the left ventricle (black arrow). On the follow-up E) non-contrast CT and F) CTP images, a new ischemic alteration is visible (blue oval). On follow-up G) conventional and H) $40 \mathrm{keV}$ monoenergetic CTA a diminution of the ventricular thrombus (black arrow) is visible, with the left atrial appendage thrombus (white arrow) unaltered. This suggests that the ventricular thrombus probably caused the recurrent brain infarction. Images with permission of Kauw et al.[193].

Another interesting application of DECT is X-ray attenuation decomposition (XAD). In $X A D$, the low- and high-energy attenuation datasets are visualized in a scatterplot. This scatterplot allows selection of tissues with specific attenuation properties, e.g. fat, soft tissue, iodine or calcium. In stroke imaging, identifying infarcted tissue attenuation properties on non-contrast CT may increase the accuracy of the initial assessment of the infarcted brain. XAD analysis can eventually be extended by adding dimensions to the scatterplot. In this way different tissues get more detailed attenuation profiles, which enhances tissue differentiation. 
In contrast with conventional or dual-energy CT, photon-counting CT (PCCT) uses an energy-integrating detector. This enables not just counting the number of incident photons, but also measuring the photon energies[194,195]. These photons are then categorized into energy bins, resulting in multiple images at different energy levels. Image noise levels will be lower because photon-counting detectors minimize electronic noise, enable optimal x-ray photon energy weighting, and provide high-resolution information that translates to lower noise in the final CT image[194,195]. PCCT even allows improved iodine contrast measurements for CTP[195,196] and might increase differentiation options using the CT images from the different energy bins between infarcted and healthy tissue for NCCT. PCCT also shows promising results in reducing the radiation dose[197,198]. Potentially, PCCT scanners can overcome some of the issues currently applicable to CT stroke imaging. There are, however, still challenges to be met such as reducing cross talk between detector elements, overcoming pile up of photon energies in detector elements, increasing the axial coverage of the detector, enlarging detector stability, and decreasing reconstruction time[194] before PCCT scanner become clinically available.

\section{Conclusion}

To conclude, this thesis has shown the potential of dual-energy CT in enhancing visualization and detection of infarctions on non-contrast CT and CT perfusion images. This can be done without increasing ionizing radiation dose and without sacrificing image quality compared with conventional CT. When Jog Mode scans are required to increase axial coverage, we have shown that the increase in acquisition interval still results in reliable CT perfusion results. To even further lower the number of missed infarcts, guided bilateral filter techniques for thin-slice CT perfusion may be used to increase the detectability of small-volume infarctions. 



\section{CHAPTER 11}

\section{BIBLIOGRAPHY}

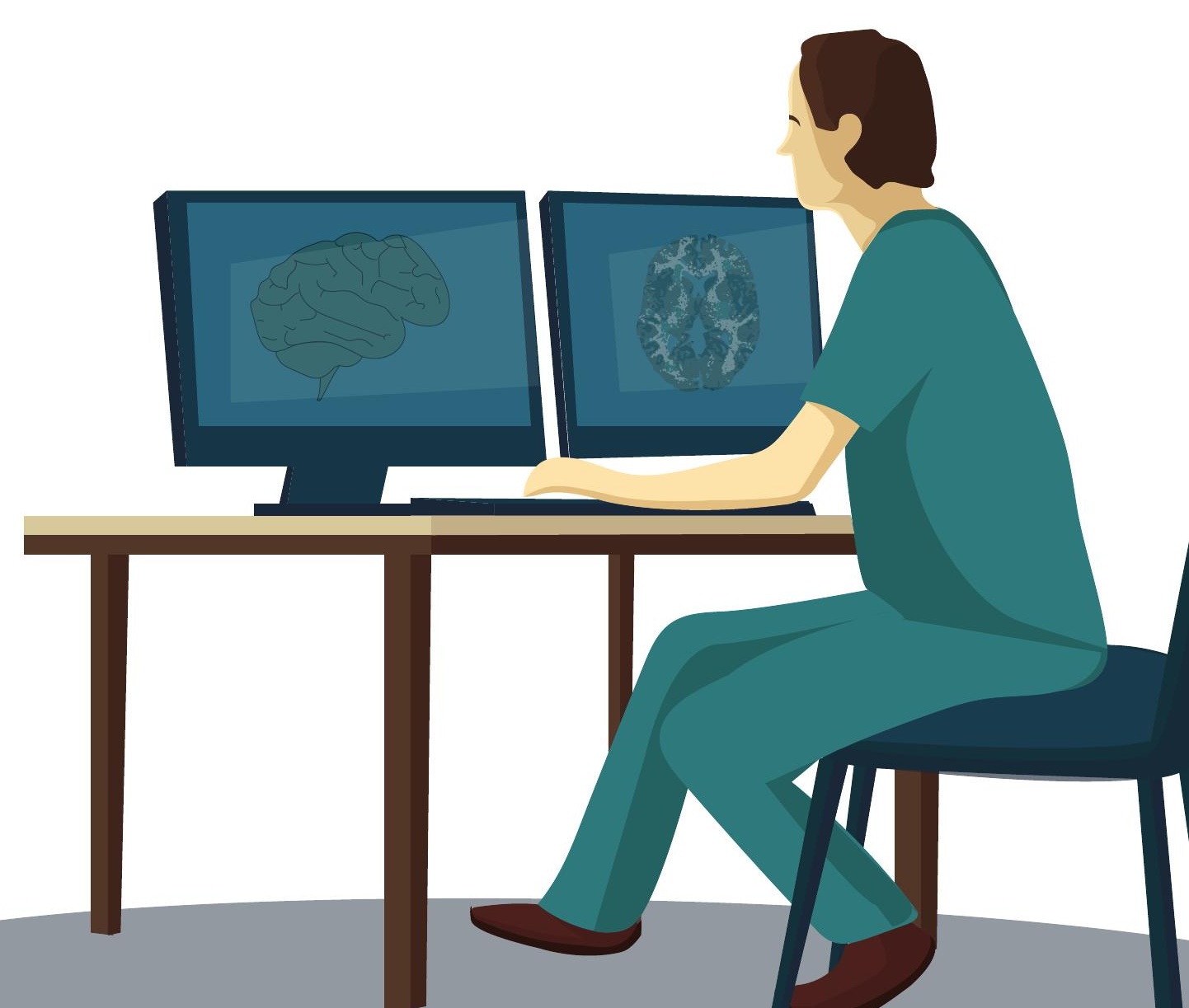


1. WHO (2017). Cardiovascular diseases (CVDs). https://www.who.int/news-room/fact-sheets/ detail/cardiovascular-diseases-(cvds). Accessed Oct. 25, 2019.

2. AR de Boer, ML Bots, I van Dis, I Vaartjes and FLJ Visseren (2018). Hart- en vaatziekten in Nederland 2018. Nederlandse Hartstichting, 1-136.

3. CJL Murray, T Vos, R Lozano, M Naghavi, AD Flaxman, C Michaud, et al. (2012). Disabilityadjusted life years (DALYs) for 291 diseases and injuries in 21 regions, 1990-2010: a systematic analysis for the Global Burden of Disease Study 2010. The Lancet, 380:2197-2223.

4. I van Dis, J Buddeke, I Vaartjes, FLJ Visseren and ML Bots (2015). Hart- en vaatziekten in Nederland 2015, cijfers over heden, verleden en toekomst. Nederlandse Hartstichting, 1-132.

5. GA Donnan, M Fisher, M Macleod and SM Davis (2008). Stroke. Lancet, 371:1612-23.

6. WJ Powers, CP Derdeyn, J Biller, CS Coffey, BL Hoh, EC Jauch, et al. (2015). 2015 American Heart Association/American Stroke Association Focused Update of the 2013 Guidelines for the Early Management of Patients With Acute Ischemic Stroke Regarding Endovascular Treatment. Stroke, 46:3020-3035.

7. W Hacke, M Kaste, E Bluhmki, M Brozman, A Dávalos, D Guidetti, et al. (2008). Thrombolysis with Alteplase 3 to 4.5 Hours after Acute Ischemic Stroke. New England Journal of Medicine, 359:1317-1329.

8. The IST-3 collaborative group (2012). The benefits and harms of intravenous thrombolysis with recombinant tissue plasminogen activator within $6 \mathrm{~h}$ of acute ischaemic stroke (the third international stroke trial [IST-3]): a randomised controlled trial. The Lancet, 379:2352-2363.

9. JL Saver, M Goyal, A van der Lugt, BK Menon, CBLM Majoie, DW Dippel, et al. (2016). Time to Treatment With Endovascular Thrombectomy and Outcomes From Ischemic Stroke: A Meta-analysis. JAMA, 316:1279.

10. WJ Powers, AA Rabinstein, T Ackerson, OM Adeoye, NC Bambakidis, K Becker, et al. (2019). Guidelines for the Early Management of Patients With Acute Ischemic Stroke: 2019 Update to the 2018 Guidelines for the Early Management of Acute Ischemic Stroke: A Guideline for Healthcare Professionals From the American Heart Association/American Stroke. Stroke, 50:344-418.

11. M Wintermark, M Luby, NM Bornstein, A Demchuk, J Fiehler, K Kudo, et al. (2015). International Survey of Acute Stroke Imaging Used to Make Revascularization Treatment Decisions. International Journal of Stroke, 10:759-762.

12. G Mair and JM Wardlaw (2014). Imaging of acute stroke prior to treatment: current practice and evolving techniques. The British Journal of Radiology, 87:20140216.

13. H Ma, BCV Campbell, MW Parsons, L Churilov, CR Levi, C Hsu, et al. (2019). Thrombolysis Guided by Perfusion Imaging up to 9 Hours after Onset of Stroke. New England Journal of Medicine, 380:1795-1803.

14. GW Albers, MP Marks, S Kemp, S Christensen, JP Tsai, S Ortega-Gutierrez, et al. (2018). Thrombectomy for Stroke at 6 to 16 Hours with Selection by Perfusion Imaging. New England Journal of Medicine, 378:708-718.

15. K Kudo, M Sasaki, K Ogasawara, S Terae, S Ehara and H Shirato (2009). Difference in Tracer Delay-induced Effect among Deconvolution Algorithms in CT Perfusion Analysis: Quantitative Evaluation with Digital Phantoms. Radiology, 251:241-249.

16. K Kudo, M Sasaki, K Yamada, S Momoshima, H Utsunomiya, H Shirato, et al. (2010). Differences in CT Perfusion Maps Generated by Different Commercial Software: Quantitative Analysis by Using Identical Source Data of Acute Stroke Patients. Radiology, 254:200-209.

17. E Bennink, J Oosterbroek, K Kudo, MA Viergever, BK Velthuis and HWAM de Jong (2016). Fast nonlinear regression method for CT brain perfusion analysis. Journal of Medical Imaging, 3:026003.

18. K Miles, M Hayball and A Dixon (1991). Colour perfusion imaging: a new application of computed tomography. The Lancet, 337:643-645.

19. A Srinivasan, M Goyal, F Al Azri and C Lum (2006). State-of-the-Art Imaging of Acute Stroke. RadioGraphics, 26:S75-S95. 
20. RC Rennert, AR Wali, JA Steinberg, DR Santiago-Dieppa, SE Olson, JS Pannell, et al. (2019). Epidemiology, Natural History, and Clinical Presentation of Large Vessel Ischemic Stroke. Neurosurgery, 85:S4-S8.

21. CH McCollough, S Leng, L Yu and JG Fletcher (2015). Dual- and Multi-Energy CT: Principles, Technical Approaches, and Clinical Applications. Radiology, 276:637-653.

22. DN Tran, M Straka, JE Roos, S Napel and D Fleischmann (2009). Dual-energy CT Discrimination of Iodine and Calcium. Academic Radiology, 16:160-171.

23. SR Pomerantz, S Kamalian, D Zhang, R Gupta, O Rapalino, D V. Sahani, et al. (2013). Virtual Monochromatic Reconstruction of Dual-Energy Unenhanced Head CT at 65-75 keV Maximizes Image Quality Compared with Conventional Polychromatic CT. Radiology, 266:318-325.

24. S Lam, R Gupta, M Levental, E Yu, HDD Curtin and R Forghani (2015). Optimal Virtual Monochromatic Images for Evaluation of Normal Tissues and Head and Neck Cancer Using Dual-Energy CT. American Journal of Neuroradiology, 36:1518-1524.

25. MPM Tijssen, PAM Hofman, AAR Stadler, W Van Zwam, R De Graaf, RJ Van Oostenbrugge, et al. (2014). The role of dual energy CT in differentiating between brain haemorrhage and contrast medium after mechanical revascularisation in acute ischaemic stroke. European Radiology, 24:834-840.

26. J Gariani, V Cuvinciuc, D Courvoisier, B Krauss, V Mendes Pereira, R Sztajzel, et al. (2016). Diagnosis of acute ischemia using dual energy CT after mechanical thrombectomy. Journal of Neurolnterventional Surgery, 8:996-1000.

27. TRC Johnson, B Krauß, M Sedlmair, M Grasruck, H Bruder, D Morhard, et al. (2007). Material differentiation by dual energy CT: Initial experience. European Radiology, 17:1510-1517.

28. MH Albrecht, TJ Vogl, SS Martin, JW Nance, TM Duguay, JL Wichmann, et al. (2019). Review of Clinical Applications for Virtual Monoenergetic Dual-Energy CT. Radiology, 293:260-271.

29. EC Lin (2010). Radiation Risk From Medical Imaging. Mayo Clinic Proceedings, 85:1142-1146.

30. RHH Wellenberg, MF Boomsma, JAC van Osch, A Vlassenbroek, J Milles, MA Edens, et al. (2017). Quantifying metal artefact reduction using virtual monochromatic dual-layer detector spectral CT imaging in unilateral and bilateral total hip prostheses. European Journal of Radiology, 88:61-70.

31. N Rassouli, H Chalian, P Rajiah, A Dhanantwari and L Landeras (2017). Assessment of 70-keV virtual monoenergetic spectral images in abdominal CT imaging: A comparison study to conventional polychromatic 120-kVp images. Abdominal Radiology, 42:2579-2586.

32. T Hickethier, J Byrtus, M Hauger, A-I luga, G Pahn, D Maintz, et al. (2018). Utilization of virtual mono-energetic images (MonoE) derived from a dual-layer spectral detector CT (SDCT) for the assessment of abdominal arteries in venous contrast phase scans. European Journal of Radiology, 99:28-33.

33. O Ozguner, A Dhanantwari, S Halliburton, G Wen, S Utrup and D Jordan (2018). Objective image characterization of a spectral CT scanner with dual-layer detector. Physics in Medicine \& Biology, 63:025027.

34. K Kalisz, N Rassouli, A Dhanantwari, D Jordan and P Rajiah (2018). Noise characteristics of virtual monoenergetic images from a novel detector-based spectral CT scanner. European Journal of Radiology, 98:118-125.

35. A Vlassenbroek (2011). Dual Layer CT. In: Dual Energy CT in Clinical Practice, Springer Berlin Heidelberg, 21-34.

36. LM Ho, TT Yoshizumi, LM Hurwitz, RC Nelson, D Marin, G Toncheva, et al. (2009). Dual Energy Versus Single Energy MDCT: Measurement of Radiation Dose Using Adult Abdominal Imaging Protocols. Academic Radiology, 16:1400-1407.

37. D Schick and J Pratap (2016). Radiation dose efficiency of dual-energy CT benchmarked against single-source, kilovoltage-optimized scans. The British Journal of Radiology, 89:20150486.

38. E McDonagh (2016). OpenREM Documentation. 1-135. 
39. DC Hoaglin, B Iglewicz and JW Tukey (1986). Performance of Some Resistant Rules for Outlier Labeling. Journal of the American Statistical Association, 81:991-999.

40. J Doerner, M Hauger, T Hickethier, J Byrtus, C Wybranski, NG Hokamp, et al. (2017). Image quality evaluation of dual-layer spectral detector CT of the chest and comparison with conventional CT imaging. European Journal of Radiology, 93:52-58.

41. M Hojjati, S Van Hedent, N Rassouli, C Tatsuoka, D Jordan, A Dhanantwari, et al. (2017). Quality of routine diagnostic abdominal images generated from a novel detector-based spectral CT scanner: a technical report on a phantom and clinical study. Abdominal Radiology, 42:2752-2759.

42. S Oda, T Nakaura, D Utsunomiya, Y Funama, N Taguchi, M Imuta, et al. (2017). Clinical potential of retrospective on-demand spectral analysis using dual-layer spectral detectorcomputed tomography in ischemia complicating small-bowel obstruction. Emergency Radiology, 24:431-434.

43. V Neuhaus, N Abdullayev, N Große Hokamp, G Pahn, C Kabbasch, A Mpotsaris, et al. (2017). Improvement of Image Quality in Unenhanced Dual-Layer CT of the Head Using Virtual Monoenergetic Images Compared With Polyenergetic Single-Energy CT. Investigative Radiology, 52:470-476.

44. RW van Hamersvelt, MJ Willemink, PA de Jong, J Milles, A Vlassenbroek, AMR Schilham, et al. (2017). Feasibility and accuracy of dual-layer spectral detector computed tomography for quantification of gadolinium: a phantom study. European Radiology, 27:3677-3686.

45. AP Sauter, FK Kopp, D Münzel, J Dangelmaier, M Renz, B Renger, et al. (2018). Accuracy of iodine quantification in dual-layer spectral CT: Influence of iterative reconstruction, patient habitus and tube parameters. European Journal of Radiology, 102:83-88.

46. Y Iyama, T Nakaura, A lyama, K Katahira and Y Yamashita (2018). The Usefulness of DualLayer Spectral Computed Tomography for Myelography: A Case Report and Review of the Literature. Case Reports in Orthopedics, 2018:1-4.

47. A Ghandour, A Sher, N Rassouli, A Dhanantwari and P Rajiah (2018). Evaluation of Virtual Monoenergetic Images on Pulmonary Vasculature Using the Dual-Layer Detector-Based Spectral Computed Tomography. Journal of Computer Assisted Tomography, 42:858-865.

48. CH Lee, JM Goo, HJ Ye, S-J Ye, CM Park, EJ Chun, et al. (2008). Radiation Dose Modulation Techniques in the Multidetector CT Era: From Basics to Practice. RadioGraphics, 28:1451-1459.

49. Fvan Ommen, E Bennink, A Vlassenbroek, JW Dankbaar, AMR Schilham, MA Viergever, et al. (2018). Image quality of conventional images of dual-layer SPECTRAL CT: A phantom study. Medical Physics, 45:3031-3042.

50. X-M Zhao, M Wang, R-Z Wu, E Dharaiya, F Feng, M-L Li, et al. (2018). Dual-layer spectral detector CT monoenergetic reconstruction improves image quality of non-contrast cerebral CT as compared with conventional single energy CT. European Journal of Radiology, 103:131-138.

51. RE Alvarez and A Macovski (1976). Energy-selective reconstructions in X-ray computerized tomography. Physics in Medicine and Biology, 21:733-44.

52. A Macovski, RE Alvarez, JLH Chan, JP Stonestrom and LM Zatz (1976). Energy dependent reconstruction in X-ray computerized tomography. Computers in Biology and Medicine, 6:325-336.

53. R Carmi, G Kafri, A Altman, L Goshen, D Planer and J Sosna (2010). Arterial doublecontrast dual-energy MDCT: in-vivo rabbit atherosclerosis with iodinated nanoparticles and gadolinium agents. In: Medical Imaging 2010: Biomedical Applications in Molecular, Structural, and Functional Imaging, SPIE, 435-443.

54. The Phantom Laboratory Inc. (2006). Catphan 500 and 600 Manual. 1-33.

55. JM Boone, JA Brink, S Edyvean, W Huda, W Leitz, CH McCollough, et al. (2012). Report 87. Journal of the ICRU, 12:1-154.

56. EL Nickoloff (1988). Measurement of the PSF for a CT scanner: appropriate wire diameter and pixel size. Physics in Medicine \& Biology, 33:149.

57. RW van Hamersvelt, AMR Schilham, K Engelke, AM den Harder, B de Keizer, HJ Verhaar, et al. (2017). Accuracy of bone mineral density quantification using dual-layer spectral detector CT: a phantom study. European Radiology, 27:4351-4359. 
58. T Hickethier, B Baeßler, JR Kroeger, J Doerner, G Pahn, D Maintz, et al. (2017). Monoenergetic reconstructions for imaging of coronary artery stents using spectral detector CT: In-vitro experience and comparison to conventional images. Journal of Cardiovascular Computed Tomography, 11:33-39.

59. O Ozguner, S Halliburton, A Dhanantwari, G Wen, S Utrup and D Jordan (2016). WE-FG207B-11: Objective Image Characterization of Spectral CT with a Dual-Layer Detector. Medical Physics, 43:3836-3836.

60. Philips Healthcare (2016). IQon Spectral CT Technical Reference Guide. 1-90.

61. LM Zatz and RE Alvarez (1977). An inaccuracy in computed tomography: the energy dependence of CT values. Radiology, 124:91-97.

62. A Löve, M-L Olsson, R Siemund, F Stålhammar, IM Björkman-Burtscher and M Söderberg (2013). Six iterative reconstruction algorithms in brain CT: a phantom study on image quality at different radiation dose levels. The British Journal of Radiology, 86:20130388.

63. D Mehta, R Thompson, T Morton, A Dhanantwari and E Shefer (2013). Iterative model reconstruction: simultaneously lowered computed tomography radiation dose and improved image quality. Medical Physics International Journal, 1:147-155.

64. MJ Willemink, RAP Takx, PA de Jong, RPJ Budde, R Bleys, M Das, et al. (2014). Computed tomography radiation dose reduction: Effect of different iterative reconstruction algorithms on image quality. Journal of Computer Assisted Tomography, 38:815-823.

65. FA Miéville, F Gudinchet, F Brunelle, FO Bochud and FR Verdun (2013). Iterative reconstruction methods in two different MDCT scanners: Physical metrics and 4-alternative forced-choice detectability experiments - A phantom approach. Physica Medica, 29:99-110.

66. MA Harris, J Huckle, D Anthony and P Charnock (2017). The Acceptability of Iterative Reconstruction Algorithms in Head CT: An Assessment of Sinogram Affirmed Iterative Reconstruction (SAFIRE) vs. Filtered Back Projection (FBP) Using Phantoms. Journal of Medical Imaging and Radiation Sciences, 48:259-269.

67. LW Goldman (2007). Principles of CT: Radiation Dose and Image Quality. Journal of Nuclear Medicine Technology, 35:213-225.

68. M Petersilka, H Bruder, B Krauss, K Stierstorfer and TG Flohr (2008). Technical principles of dual source CT. European Journal of Radiology, 68:362-368.

69. R Gupta, CM Phan, C Leidecker, TJ Brady, JA Hirsch, RG Nogueira, et al. (2010). Evaluation of Dual-Energy CT for Differentiating Intracerebral Hemorrhage from lodinated Contrast Material Staining. Radiology, 257:205-211.

70. L Ananthakrishnan, P Rajiah, R Ahn, N Rassouli, YXi, TC Soesbe, et al. (2017). Spectral detector CT-derived virtual non-contrast images: comparison of attenuation values with unenhanced CT. Abdominal Radiology, 42:702-709.

71. H Chandarana, AJ Megibow, BA Cohen, R Srinivasan, D Kim, C Leidecker, et al. (2011). Iodine quantification with dual-energy CT: Phantom study and preliminary experience with renal masses. American Journal of Roentgenology, 196:693-700.

72. KD Song, CK Kim, BK Park and B Kim (2011). Utility of iodine overlay technique and virtual unenhanced images for the characterization of renal masses by dual-energy CT. American Journal of Roentgenology, 197:1076-1082.

73. C Zhou, YE Zhao, S Luo, H Shi, L Li, L Zheng, et al. (2011). Monoenergetic Imaging of Dualenergy CT Reduces Artifacts from Implanted Metal Orthopedic Devices in Patients with Factures. Academic Radiology, 18:1252-1257.

74. Y Dong, AJ Shi, JL Wu, RX Wang, LF Sun, AL Liu, et al. (2016). Metal artifact reduction using virtual monochromatic images for patients with pedicle screws implants on CT. European Spine Journal, 25:1754-1763.

75. DC Jung, YT Oh, MD Kim and M Park (2012). Usefulness of the Virtual Monochromatic Image in Dual-Energy Spectral CT for Decreasing Renal Cyst Pseudoenhancement: A Phantom Study. American Journal of Roentgenology, 199:1316-1319. 
76. Y Yamada, M Jinzaki, Y Tanami, T Abe and S Kuribayashi (2012). Virtual Monochromatic Spectral Imaging for the Evaluation of Hypovascular Hepatic Metastases. Investigative Radiology, 47:292-298.

77. E Pessis, R Campagna, J-M Sverzut, F Bach, M Rodallec, H Guerini, et al. (2013). Virtual Monochromatic Spectral Imaging with Fast Kilovoltage Switching: Reduction of Metal Artifacts at CT. RadioGraphics, 33:573-583.

78. WD Hwang, M Mossa-Basha, JB Andre, DS Hippe, S Culbertson and Y Anzai (2016). Qualitative Comparison of Noncontrast Head Dual-Energy Computed Tomography Using Rapid Voltage Switching Technique and Conventional Computed Tomography. Journal of Computer Assisted Tomography, 40:320-325.

79. CA Potter and AD Sodickson (2016). Dual-Energy CT in Emergency Neuroimaging: Added Value and Novel Applications. RadioGraphics, 36:2186-2198.

80. J Park, YH Choi, JE Cheon, WS Kim, IO Kim, SY Pak, et al. (2017). Advanced virtual monochromatic reconstruction of dual-energy unenhanced brain computed tomography in children: comparison of image quality against standard mono-energetic images and conventional polychromatic computed tomography. Pediatric Radiology, 47:1648-1658.

81. C Rozeik, O Kotterer, J Preiss, M Schütz, W Dingler and HK Deininger (1991). Cranial CT Artifacts and Gantry Angulation. Journal of Computer Assisted Tomography, 15:381-386.

82. HR Hixson, C Leiva-Salinas, S Sumer, J Patrie, W Xin and M Wintermark (2016). Utilizing dual energy CT to improve CT diagnosis of posterior fossa ischemia. Journal of Neuroradiology, 43:346-352.

83. M Wintermark, PC Sanelli, GW Albers, J Bello, C Derdeyn, SW Hetts, et al. (2013). Imaging Recommendations for Acute Stroke and Transient Ischemic Attack Patients: A Joint Statement by the American Society of Neuroradiology, the American College of Radiology, and the Society of Neurolnterventional Surgery. American Journal of Neuroradiology, 34:117-127.

84. JM Wardlaw and O Mielke (2005). Early signs of brain infarction at CT: Observer reliability and outcome after thrombolytic treatment - Systematic review. Radiology, 235:444-453.

85. KW Muir, A Buchan, R von Kummer, J Rother and J-C Baron (2006). Imaging of acute stroke. The Lancet Neurology, 5:755-768.

86. JG Merino and S Warach (2010). Imaging of acute stroke. Nature Reviews Neurology, 6:560-571.

87. DN Wolman, BP Patel, M Wintermark and JJ Heit (2018). Dual-Energy Computed Tomography Applications in Neurointervention. Journal of Computer Assisted Tomography, 42:831-839.

88. L Yu, JA Christner, S Leng, J Wang, JG Fletcher and CH McCollough (2011). Virtual monochromatic imaging in dual-source dual-energy CT: Radiation dose and image quality. Medical Physics, 38:6371-6379.

89. K Taguchi, T Itoh, MK Fuld, E Fournie, O Lee and K Noguchi (2018). "X-Map 2.0" for Edema Signal Enhancement for Acute Ischemic Stroke Using Non-Contrast-Enhanced Dual-Energy Computed Tomography. Investigative Radiology, 53:432-439.

90. AE Grams, T Djurdjevic, R Rehwald, T Schiestl, F Dazinger, R Steiger, et al. (2018). Improved visualisation of early cerebral infarctions after endovascular stroke therapy using dualenergy computed tomography oedema maps. European Radiology, 28:4534-4541.

91. MF Mohammed, O Marais, A Min, D Ferguson, S Jalal, F Khosa, et al. (2018). Unenhanced Dual-Energy Computed Tomography. Investigative Radiology, 53:63-69.

92. PA Barber, AM Demchuk, J Zhang and AM Buchan (2000). Validity and reliability of a quantitative computed tomography score in predicting outcome of hyperacute stroke before thrombolytic therapy. The Lancet, 355:1670-1674.

93. V Puetz, PN Sylaja, SB Coutts, MD Hill, I Dzialowski, P Mueller, et al. (2008). Extent of Hypoattenuation on CT Angiography Source Images Predicts Functional Outcome in Patients With Basilar Artery Occlusion. Stroke, 39:2485-2490.

94. JE Park, YH Choi, JE Cheon, WS Kim, IO Kim, HS Cho, et al. (2017). Image quality and radiation dose of brain computed tomography in children: effects of decreasing tube voltage from 120 kVp to 80 kVp. Pediatric Radiology, 47:710-717. 
95. M Yoshida, T Nakaura, S Tanoue, S Takada, T Inoue, H Uetani, et al. (2019). Dual-Energy Computed Tomography for Evaluating Acute Brain Infarction of Middle Cerebral Artery Territories. Journal of Computer Assisted Tomography, 43:460-466.

96. WJ Powers, AA Rabinstein, T Ackerson, OM Adeoye, NC Bambakidis, K Becker, et al. (2018). 2018 Guidelines for the Early Management of Patients With Acute Ischemic Stroke: A Guideline for Healthcare Professionals From the American Heart Association/American Stroke Association. Stroke, 49:e46-e110.

97. CM Phan, AJ Yoo, JA Hirsch, RG Nogueira and R Gupta (2012). Differentiation of Hemorrhage from lodinated Contrast in Different Intracranial Compartments Using Dual-Energy Head CT. American Journal of Neuroradiology, 33:1088-1094.

98. MG Lansberg, M Straka, S Kemp, M Mlynash, LR Wechsler, TG Jovin, et al. (2012). MRI profile and response to endovascular reperfusion after stroke (DEFUSE 2): a prospective cohort study. The Lancet Neurology, 11:860-867.

99. RG Nogueira, AP Jadhav, DC Haussen, A Bonafe, RF Budzik, P Bhuva, et al. (2018). Thrombectomy 6 to 24 Hours after Stroke with a Mismatch between Deficit and Infarct. New England Journal of Medicine, 378:11-21.

100. MP Marks, MG Lansberg, M Mlynash, S Kemp, R McTaggart, G Zaharchuk, et al. (2014). Correlation of AOL recanalization, TIMI reperfusion and TICI reperfusion with infarct growth and clinical outcome. Journal of NeuroInterventional Surgery, 6:724-728.

101. GW Albers, M Goyal, R Jahan, A Bonafe, H-C Diener, El Levy, et al. (2016). Ischemic core and hypoperfusion volumes predict infarct size in SWIFT PRIME. Annals of Neurology, 79:76-89.

102. MP Marks, MG Lansberg, M Mlynash, S Kemp, RA McTaggart, G Zaharchuk, et al. (2014). Angiographic Outcome of Endovascular Stroke Therapy Correlated with MR Findings, Infarct Growth, and Clinical Outcome in the DEFUSE 2 Trial. International Journal of Stroke, 9:860-865.

103. JJ Heit and M Wintermark (2016). Perfusion Computed Tomography for the Evaluation of Acute Ischemic Stroke. Stroke, 47:1153-1158.

104. TY Wu, O Sobowale, R Hurford, G Sharma, S Christensen, N Yassi, et al. (2016). Software output from semi-automated planimetry can underestimate intracerebral haemorrhage and peri-haematomal oedema volumes by up to $41 \%$. Neuroradiology, 58:867-876.

105. D Giavarina (2015). Understanding Bland Altman analysis. Biochemia Medica, 25:141-151.

106. JM Bland and DG Altman (1999). Measuring agreement in method comparison studies. Statistical Methods in Medical Research, 8:135-160.

107. JL Saver, M Goyal, A Bonafe, HC Diener, El Levy, VM Pereira, et al. (2015). Stent-retriever thrombectomy after intravenous t-PA vs. t-PA alone in stroke. New England Journal of Medicine, 372:2285-2295.

108. BCV Campbell, PJ Mitchell, TJ Kleinig, HM Dewey, L Churilov, N Yassi, et al. (2015). Endovascular Therapy for Ischemic Stroke with Perfusion-Imaging Selection. New England Journal of Medicine, 372:1009-1018.

109. CW Cereda, S Christensen, BCV Campbell, NK Mishra, M Mlynash, C Levi, et al. (2016). A benchmarking tool to evaluate computer tomography perfusion infarct core predictions against a DWI standard. Journal of Cerebral Blood Flow \& Metabolism, 36:1780-1789.

110. B Farzin, R Fahed, F Guilbert, AY Poppe, N Daneault, AP Durocher, et al. (2016). Early CT changes in patients admitted for thrombectomy. Neurology, 87:249-256.

111. AJ Yoo, OO Zaidat, ZA Chaudhry, OA Berkhemer, RG González, M Goyal, et al. (2014). Impact of Pretreatment Noncontrast CT Alberta Stroke Program Early CT Score on Clinical Outcome After Intra-Arterial Stroke Therapy. Stroke, 45:746-751.

112. S Prakkamakul and AJ Yoo (2017). ASPECTS CT in Acute Ischemia. Topics in Magnetic Resonance Imaging, 26:103-112.

113. G Broocks, F Flottmann, A Scheibel, A Aigner, TD Faizy, U Hanning, et al. (2018). Quantitative lesion water uptake in acute stroke computed tomography is a predictor of malignant infarction. Stroke, 49:1906-1912.

114. K-A Hossmann (2008). Cerebral ischemia: Models, methods and outcomes. Neuropharmacology, 55:257-270. 
115. X Liu, L Yu, AN Primak and CH McCollough (2009). Quantitative imaging of element composition and mass fraction using dual-energy CT: Three-material decomposition. Medical Physics, 36:1602-1609.

116. R von Kummer and I Dzialowski (2017). Imaging of cerebral ischemic edema and neuronal death. Neuroradiology, 59:545-553.

117. J Minnerup, G Broocks, J Kalkoffen, S Langner, M Knauth, MN Psychogios, et al. (2016). Computed tomography-based quantification of lesion water uptake identifies patients within 4.5 hours of stroke onset: A multicenter observational study. Annals of Neurology, 80:924-934.

118. C Kobkitsuksakul, O Tritanon and V Suraratdecha (2018). Interobserver agreement between senior radiology resident, neuroradiology fellow, and experienced neuroradiologist in the rating of Alberta Stroke Program Early Computed Tomography Score (ASPECTS). Diagnostic and Interventional Radiology, 24:104-107.

119. BCV Campbell, CBLM Majoie, GW Albers, BK Menon, N Yassi, G Sharma, et al. (2019). Penumbral imaging and functional outcome in patients with anterior circulation ischaemic stroke treated with endovascular thrombectomy versus medical therapy: a meta-analysis of individual patient-level data. The Lancet Neurology, 18:46-55.

120. TE Mayer, GF Hamann, J Baranczyk, B Rosengarten, E Klotz, M Wiesmann, et al. (2000). Dynamic CT perfusion imaging of acute stroke. American Journal of Neuroradiology, 21:1441-1449.

121. M Wintermark, JP Thiran, P Maeder, P Schnyder and R Meuli (2001). Simultaneous measurement of regional cerebral blood flow by perfusion CT and stable xenon CT: A validation study. American Journal of Neuroradiology, 22:905-914.

122. M Wintermark, NJ Fischbein, WS Smith, NU Ko, M Quist and WP Dillon (2005). Accuracy of dynamic perfusion CT with deconvolution in detecting acute hemispheric stroke. American Journal of Neuroradiology, 26:104-112.

123. B Abels, E Klotz, BF Tomandl, SP Kloska and MM Lell (2010). Perfusion CT in acute ischemic stroke: A qualitative and quantitative comparison of deconvolution and maximum slope approach. American Journal of Neuroradiology, 31:1690-1698.

124. UP Fulwadhva, JR Wortman and AD Sodickson (2016). Use of Dual-Energy CT and lodine Maps in Evaluation of Bowel Disease. RadioGraphics, 36:393-406.

125. G Landry, B Reniers, PV Granton, B van Rooijen, L Beaulieu, JE Wildberger, et al. (2011). Extracting atomic numbers and electron densities from a dual source dual energy CT scanner: Experiments and a simulation model. Radiotherapy and Oncology, 100:375-379.

126. B Krauss, B Schmidt and TG Flohr (2011). Dual Source CT. In: Dual Energy CT in Clinical Practice, Springer Berlin Heidelberg, 11-20.

127. N Chandra and DA Langan (2011). Gemstone Detector: Dual Energy Imaging via Fast kVp Switching. In: Dual Energy CT in Clinical Practice, Springer Berlin Heidelberg, 35-41.

128. F van Ommen, HWAM de Jong, JW Dankbaar, E Bennink, T Leiner and AMR Schilham (2019). Dose of CT protocols acquired in clinical routine using a dual-layer detector CT scanner: A preliminary report. European Journal of Radiology, 112:65-71.

129. S Klein, M Staring, K Murphy, MA Viergever and J Pluim (2010). elastix: A Toolbox for IntensityBased Medical Image Registration. IEEE Transactions on Medical Imaging, 29:196-205.

130. HM Silvennoinen, LM Hamberg, L Valanne and GJ Hunter (2007). Increasing Contrast Agent Concentration Improves Enhancement in First-Pass CT Perfusion. American Journal of Neuroradiology, 28:1299-1303.

131. M Wintermark, WS Smith, NU Ko, M Quist, P Schnyder and WP Dillon (2004). Dynamic Perfusion CT: Optimizing the Temporal Resolution and Contrast Volume for Calculation of Perfusion CT Parameters in Stroke Patients. American Journal of Neuroradiology, 25:720-729.

132. JM Niesten, IC Van Der Schaaf, AJ Riordan, HWAM De Jong, AD Horsch, D Eijspaart, et al. (2014). Radiation dose reduction in cerebral CT perfusion imaging using iterative reconstruction. European Radiology, 24:484-493.

133. K-O Lövblad and AE Baird (2010). Computed tomography in acute ischemic stroke. Neuroradiology, 52:175-187. 
134. EM de Lucas, E Sánchez, A Gutiérrez, AG Mandly, E Ruiz, AF Flórez, et al. (2008). CT Protocol for Acute Stroke: Tips and Tricks for General Radiologists. RadioGraphics, 28:1673-1687.

135. BJ Emmer, M Rijkee, JM Niesten, MJH Wermer, BK Velthuis and MAA van Walderveen (2014). Whole brain CT perfusion in acute anterior circulation ischemia: coverage size matters. Neuroradiology, 56:1121-1126.

136. JM Biesbroek, JM Niesten, JW Dankbaar, GJ Biessels, BK Velthuis, JB Reitsma, et al. (2013). Diagnostic accuracy of CT perfusion imaging for detecting acute ischemic stroke: A systematic review and meta-analysis. Cerebrovascular Diseases, 35:493-501.

137. A Kämena, F Streitparth, C Grieser, L Lehmkuhl, B Jamil, K Wojtal, et al. (2007). Dynamic perfusion CT: Optimizing the temporal resolution for the calculation of perfusion CT parameters in stroke patients. European Journal of Radiology, 64:111-118.

138. M Wiesmann, S Berg, G Bohner, R Klingebiel, V Schöpf, BM Stoeckelhuber, et al. (2008). Dose reduction in dynamic perfusion CT of the brain: Effects of the scan frequency on measurements of cerebral blood flow, cerebral blood volume, and mean transit time. European Radiology, 18:2967-2974.

139. B Abels, E Klotz, BF Tomandl, JP Villablanca, SP Kloska and MM Lell (2011). CT perfusion in acute ischemic stroke: A comparison of 2-second and 1-second temporal resolution. American Journal of Neuroradiology, 32:1632-1639.

140. G Cao, W Chen, H Sun, X Guo, Y Yang, K Tang, et al. (2017). Whole-brain CT perfusion imaging using increased sampling intervals: A pilot study. Experimental and Therapeutic Medicine, 14:2643-2649.

141. O Wu, L Østergaard, RM Weisskoff, T Benner, BR Rosen and AG Sorensen (2003). Tracer arrival timing-insensitive technique for estimating flow in MR perfusion-weighted imaging using singular value decomposition with a block-circulant deconvolution matrix. Magnetic Resonance in Medicine, 50:164-174.

142. AJ Riordan, M Prokop, MA Viergever, JW Dankbaar, EJ Smit and HWAM de Jong (2011). Validation of CT brain perfusion methods using a realistic dynamic head phantom. Medical Physics, 38:3212-3221.

143. LR Dice (1945). Measures of the Amount of Ecologic Association Between Species. Ecology, 26:297-302.

144. JM Wardlaw (2005). What causes lacunar stroke? Journal of Neurology, Neurosurgery \& Psychiatry, 76:617-619.

145. L Lin, A Bivard and MW Parsons (2013). Perfusion Patterns of Ischemic Stroke on Computed Tomography Perfusion. Journal of Stroke, 15:164.

146. CLM Sudlow and CP Warlow (1997). Comparable Studies of the Incidence of Stroke and its Pathological Types. Stroke, 28:491-499.

147. S Sacco, C Marini, R Totaro, T Russo, D Cerone and A Carolei (2006). A population-based study of the incidence and prognosis of lacunar stroke. Neurology, 66:1335-1338.

148. DS Wack, KV. Snyder, KF Seals and AH Siddiqui (2014). Masked smoothing using separable kernels for CT perfusion images. BMC Medical Imaging, 14:28.

149. AM Mendrik, E Vonken, B van Ginneken, HWAM de Jong, A Riordan, T van Seeters, et al. (2011). TIPS bilateral noise reduction in 4D CT perfusion scans produces high-quality cerebral blood flow maps. Physics in Medicine and Biology, 56:3857-3872.

150. F Pisana, T Henzler, S Schönberg, E Klotz, B Schmidt and M Kachelrieß (2017). Noise reduction and functional maps image quality improvement in dynamic CT perfusion using a new k-means clustering guided bilateral filter (KMGB). Medical Physics, 44:3464-3482.

151. A Mendrik, E Vonken, JW Dankbaar, M Prokop and B van Ginneken (2010). Noise filtering in thin-slice 4D cerebral CT perfusion scans. In: Medical Imaging 2010: Image Processing, $76230 \mathrm{~N}$.

152. C Tomasi and R Manduchi (1998). Bilateral filtering for gray and color images. In: Sixth International Conference on Computer Vision (IEEE Cat. No.98CH36271), Narosa Publishing House, 839-846. 
153. B Aubert-Broche, AC Evans and L Collins (2006). A new improved version of the realistic digital brain phantom. Neurolmage, 32:138-145.

154. J Bredno, ME Olszewski and M Wintermark (2010). Simulation model for contrast agent dynamics in brain perfusion scans. Magnetic Resonance in Medicine, 64:280-290.

155. AJ Britten, M Crotty, H Kiremidjian, A Grundy and EJ Adam (2004). The addition of computer simulated noise to investigate radiation dose and image quality in images with spatial correlation of statistical noise: An example application to X-ray CT of the brain. British Journal of Radiology, 77:323-328.

156. BCV Campbell, S Christensen, CR Levi, PM Desmond, GA Donnan, SM Davis, et al. (2011). Cerebral Blood Flow Is the Optimal CT Perfusion Parameter for Assessing Infarct Core. Stroke, 42:3435-3440.

157. S Kamalian, S Kamalian, MB Maas, G V. Goldmacher, S Payabvash, A Akbar, et al. (2011). CT Cerebral Blood Flow Maps Optimally Correlate With Admission Diffusion-Weighted Imaging in Acute Stroke but Thresholds Vary by Postprocessing Platform. Stroke, 42:1923-1928.

158. F van Ommen, F Kauw, E Bennink, JJ Heit, DN Wolman, JW Dankbaar, et al. (2020). Image Quality of Virtual Monochromatic Reconstructions of Noncontrast CT on a Dual-Source CT Scanner in Adult Patients. Academic Radiology, in press.

159. D Morhard, C Fink, A Graser, MF Reiser, C Becker and TRC Johnson (2009). Cervical and Cranial Computed Tomographic Angiography With Automated Bone Removal. Investigative Radiology, 44:293-297.

160. C Thomas, A Korn, B Krauss, D Ketelsen, I Tsiflikas, A Reimann, et al. (2010). Automatic bone and plaque removal using dual energy $\mathrm{CT}$ for head and neck angiography: Feasibility and initial performance evaluation. European Journal of Radiology, 76:61-67.

161. K Uotani, Y Watanabe, M Higashi, T Nakazawa, AK Kono, Y Hori, et al. (2009). Dual-energy CT head bone and hard plaque removal for quantification of calcified carotid stenosis: Utility and comparison with digital subtraction angiography. European Radiology, 19:2060-2065.

162. Y Watanabe, K Uotani, T Nakazawa, M Higashi, N Yamada, Y Hori, et al. (2009). Dual-energy direct bone removal CT angiography for evaluation of intracranial aneurysm or stenosis: comparison with conventional digital subtraction angiography. European Radiology, 19:1019-1024.

163. L-J Zhang, S-Y Wu, CS Poon, Y-E Zhao, X Chai, C-S Zhou, et al. (2010). Automatic Bone Removal Dual-Energy CT Angiography for the Evaluation of Intracranial Aneurysms. Journal of Computer Assisted Tomography, 34:816-824.

164. L-J Zhang, S-Y Wu, J-B Niu, Z-L Zhang, HZ Wang, Y-E Zhao, et al. (2010). Dual-Energy CT Angiography in the Evaluation of Intracranial Aneurysms: Image Quality, Radiation Dose, and Comparison With 3D Rotational Digital Subtraction Angiography. American Journal of Roentgenology, 194:23-30.

165. S Bisdas, F Donnerstag, B Ahl, I Bohrer, K Weissenborn and H Becker (2004). Comparison of Perfusion Computed Tomography With Diffusion-Weighted Magnetic Resonance Imaging in Hyperacute Ischemic Stroke. Journal of Computer Assisted Tomography, 28:747-755.

166. M Wintermark, R Meuli, P Browaeys, M Reichhart, J Bogousslavsky, P Schnyder, et al. (2007). Comparison of CT perfusion and angiography and MRI in selecting stroke patients for acute treatment. Neurology, 68:694-697.

167. J Demeestere, C Garcia-Esperon, P Garcia-Bermejo, F Ombelet, P McElduff, A Bivard, et al. (2017). Evaluation of hyperacute infarct volume using ASPECTS and brain CT perfusion core volume. Neurology, 88:2248-2253.

168. MC Jacobsen, D Schellingerhout, CA Wood, EP Tamm, MC Godoy, J Sun, et al. (2018). Intermanufacturer Comparison of Dual-Energy CT Iodine Quantification and Monochromatic Attenuation: A Phantom Study. Radiology, 287:224-234.

169. T Sellerer, PB Noël, M Patino, A Parakh, S Ehn, S Zeiter, et al. (2018). Dual-energy CT: a phantom comparison of different platforms for abdominal imaging. European Radiology, 28:2745-2755.

170. S Paris and F Durand (2009). A Fast Approximation of the Bilateral Filter Using a Signal Processing Approach. International Journal of Computer Vision, 81:24-52. 
171. KN Chaudhury and SD Dabhade (2016). Fast and Provably Accurate Bilateral Filtering. IEEE Transactions on Image Processing, 25:2519-2528.

172. CH McCollough, AN Primak, N Braun, J Kofler, L Yu and J Christner (2009). Strategies for Reducing Radiation Dose in CT. Radiologic Clinics of North America, 47:27-40.

173. L Yu, X Liu, S Leng, JM Kofler, JC Ramirez-Giraldo, M Qu, et al. (2009). Radiation dose reduction in computed tomography: techniques and future perspective. Imaging in Medicine, 1:65-84.

174. EP Tamm, XJ Rong, DD Cody, RD Ernst, NE Fitzgerald and V Kundra (2011). Quality Initiatives: CT Radiation Dose Reduction: How to Implement Change without Sacrificing Diagnostic Quality. RadioGraphics, 31:1823-1832.

175. L Liu (2014). Model-based Iterative Reconstruction: A Promising Algorithm for Today's Computed Tomography Imaging. Journal of Medical Imaging and Radiation Sciences, 45:131-136.

176. MJ Willemink, T Leiner, PA de Jong, LM de Heer, RAJ Nievelstein, AMR Schilham, et al. (2013). Iterative reconstruction techniques for computed tomography part 2: initial results in dose reduction and image quality. European Radiology, 23:1632-1642.

177. S Richard, DB Husarik, G Yadava, SN Murphy and E Samei (2012). Towards task-based assessment of CT performance: System and object MTF across different reconstruction algorithms. Medical Physics, 39:4115-4122.

178. BK Menon, CD D’Esterre, EM Qazi, M Almekhlafi, L Hahn, AM Demchuk, et al. (2015). Multiphase CT Angiography: A New Tool for the Imaging Triage of Patients with Acute Ischemic Stroke. Radiology, 275:510-520.

179. M Goyal, AM Demchuk, BK Menon, M Eesa, JL Rempel, J Thornton, et al. (2015). Randomized Assessment of Rapid Endovascular Treatment of Ischemic Stroke. New England Journal of Medicine, 372:1019-1030.

180. Z Wang, J Xie, T-Y Tang, C-H Zeng, Y Zhang, Z Zhao, et al. (2020). Collateral Status at SinglePhase and Multiphase CT Angiography versus CT Perfusion for Outcome Prediction in Anterior Circulation Acute Ischemic Stroke. Radiology, 296:393-400.

181. H Tian, C Chen, C Garcia-Esperon, MW Parsons, L Lin, CR Levi, et al. (2019). Dynamic CT but Not Optimized Multiphase CT Angiography Accurately Identifies CT Perfusion Target Mismatch Ischemic Stroke Patients. Frontiers in Neurology, 10:1130.

182. YW Lui, ER Tang, AM Allmendinger and V Spektor (2010). Evaluation of CT perfusion in the setting of cerebral ischemia: Patterns and pitfalls. American Journal of Neuroradiology, 31:1552-1563.

183. R Smith-Bindman (2009). Radiation Dose Associated With Common Computed Tomography Examinations and the Associated Lifetime Attributable Risk of Cancer. Archives of Internal Medicine, 169:2078-2086.

184. MW Parsons (2008). Perfusion CT: Is it Clinically Useful? International Journal of Stroke, 3:41-50.

185. R Smith-Bindman, Y Wang, P Chu, R Chung, AJ Einstein, J Balcombe, et al. (2019). International variation in radiation dose for computed tomography examinations: prospective cohort study. BMJ, 364:k4931.

186. AA Konstas, G V. Goldmakher, TY Lee and MH Lev (2009). Theoretic basis and technical implementations of CT perfusion in acute ischemic stroke, Part 2: Technical implementations. American Journal of Neuroradiology, 30:885-892.

187. CLEOPATRA-investigators (2020). Cost Effectiveness of CT Perfusion for Patients suffering from Acute Ischemic Stroke. https://www.trialregister.nl/trial/7974. Accessed Jun. 17, 2020.

188. K Noguchi, T Itoh, N Naruto, S Takashima, K Tanaka and S Kuroda (2017). A Novel Imaging Technique (X-Map) to Identify Acute Ischemic Lesions Using Noncontrast Dual-Energy Computed Tomography. Journal of Stroke and Cerebrovascular Diseases, 26:34-41.

189. J Hur, YJ Kim, H-J Lee, JE Nam, YJ Hong, HY Kim, et al. (2012). Cardioembolic Stroke: DualEnergy Cardiac CT for Differentiation of Left Atrial Appendage Thrombus and Circulatory Stasis. Radiology, 263:688-695.

190. A Korn, B Bender, C Thomas, S Danz, M Fenchel, T Nägele, et al. (2011). Dual energy CTA of the carotid bifurcation: Advantage of plaque subtraction for assessment of grade of the stenosis and morphology. European Journal of Radiology, 80:120-125. 
191. DR Obaid, PA Calvert, D Gopalan, RA Parker, NEJ West, M Goddard, et al. (2014). Dualenergy computed tomography imaging to determine atherosclerotic plaque composition: A prospective study with tissue validation. Journal of Cardiovascular Computed Tomography, 8:230-237.

192. M Das, T Braunschweig, G Mühlenbruch, AH Mahnken, T Krings, S Langer, et al. (2009). Carotid Plaque Analysis: Comparison of Dual-Source Computed Tomography (CT) Findings and Histopathological Correlation. European Journal of Vascular and Endovascular Surgery, 38:14-19.

193. F Kauw, JW Dankbaar, J Habets, MJM Cramer, HWAM de Jong, BK Velthuis, et al. (2018). A Change of Heart: Yield of Cardiac Imaging in Acute Stroke Workup. Case Reports in Neurology, 10:118-123.

194. MJ Willemink, M Persson, A Pourmorteza, NJ Pelc and D Fleischmann (2018). Photon-counting CT: Technical Principles and Clinical Prospects. Radiology, 289:293-312.

195. S Leng, M Bruesewitz, S Tao, K Rajendran, AF Halaweish, NG Campeau, et al. (2019). Photoncounting Detector CT: System Design and Clinical Applications of an Emerging Technology. RadioGraphics, 39:729-743.

196. A Tao, R Huang, S Tao, GJ Michalak, CH McCollough and S Leng (2019). Dual-source photon counting detector CT with a tin filter: a phantom study on iodine quantification performance. Physics in Medicine \& Biology, 64:115019.

197. Z Yu, S Leng, Z Li, AF Halaweish, S Kappler, EL Ritman, et al. (2016). How Low Can We Go in Radiation Dose for the Data-Completion Scan on a Research Whole-Body Photon-Counting Computed Tomography System. Journal of Computer Assisted Tomography, 40:663-670.

198. K Rajendran, BA Voss, W Zhou, S Tao, DR DeLone, JI Lane, et al. (2020). Dose Reduction for Sinus and Temporal Bone Imaging Using Photon-Counting Detector CT With an Additional Tin Filter. Investigative Radiology, 55:91-100. 
Bibliography | Chapter 11 



\section{APPENDICES}

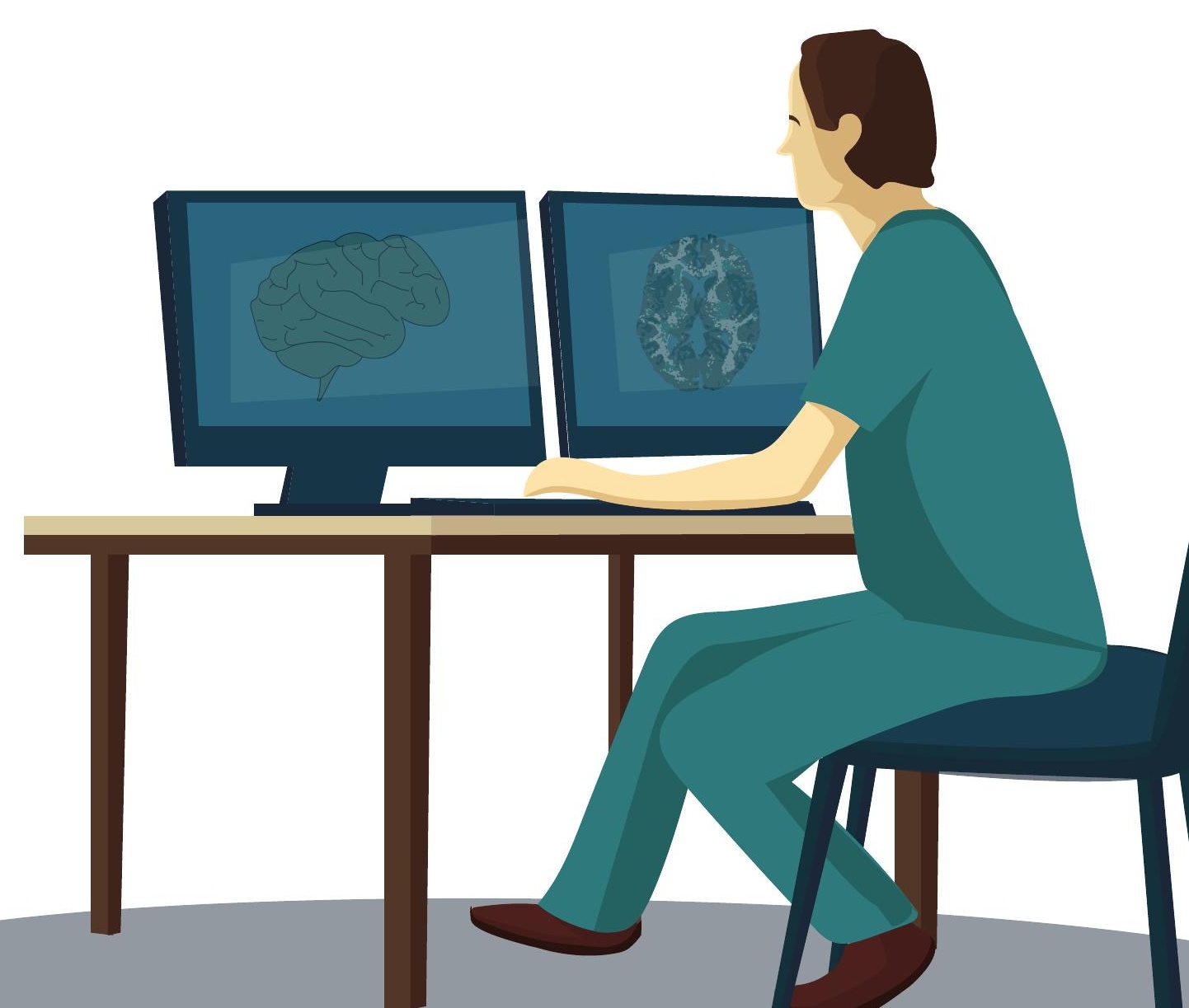




\section{Appendices}

\section{Nederlandse samenvatting}

Hart- en vaatziekten zijn wereldwijd de voornaamste doodsoorzaak. Het overgrote deel van deze sterfgevallen word veroorzaakt door een hartinfarct of een beroerte. Hierin is beroerte een verzamelnaam voor een ziektebeeld waarbij de bloedvoorziening van de hersenen (tijdelijk) is aangedaan. Er wordt onderscheid gemaakt tussen een herseninfarct en een hersenbloeding. Bij een herseninfarct sluit een bloedprop een bloedvat (tijdelijk) af, en bij een hersenbloeding is er een bloedvat in de hersenen opengebarsten. Dit proefschrift focust zich op herseninfarcten.

Er zijn grofweg twee behandelmogelijkheden voor een patiënt met een herseninfarct; er kan stolsel-oplossende medicatie worden toegediend of er wordt een zogenoemde trombectomie uitgevoerd. Bij een trombectomie wordt de bloedprop verwijderd via een katheter. Voor beide behandelingen geldt dat de behandeling binnen een bepaalde tijd na de eerste symptomen moet worden gestart, omdat na deze tijd de kans op schade groter is dan de kans op het gewenste effect. Daarom is een veelgehoorde term bij een herseninfarct "tijd is brein". Hiermee wordt bedoeld dat de diagnose en behandeling zo snel mogelijk na het ontstaan van de eerste symptomen moet zijn om zoveel mogelijk aangedaan hersenweefsel te redden. Dit is ook de reden waarom CT de voorkeur krijgt voor beeldvorming van beroertes. CT is namelijk snel, relatief goedkoop en vrijwel in elk ziekenhuis aanwezig. Het acute CT beroerte scanprotocol bestaat meestal uit een CT scan zonder contrastmiddel (NCCT), een CT perfusie (CTP) scan en een CT angiografie (CTA) scan.

Met de NCCT wordt het hele brein afgebeeld. De NCCT wordt gebruikt om te kijken of er een hersenbloeding is. Daarnaast wordt er alvast gekeken naar eerste tekenen van infarcering van de hersenen. Vervolgens wordt er een CTP scan gemaakt. Bij een CTP acquisitie wordt er jodiumhoudend contrastmiddel ingespoten. Door ongeveer een minuut lang elke één a twee seconden een nieuwe CT scan te maken van hetzelfde stuk brein kunnen we volgen hoe het contrastmiddel zich in de hersenen verspreid. Deze dynamische CT opname kunnen we dan gebruiken om de doorbloeding (perfusie) van de hersenen te bepalen. Hiermee wordt het mogelijk om van een herseninfarct te zeggen wat de infarct-kern (al gestorven hersenweefsel) is, welk omringend weefsel aangedaan is (penumbra), en welk weefsel nog gezond is. De ratio tussen het volume van infarct-kern en de penumbra is een belangrijke meetwaarde om patiënten te selecteren voor een bepaalde behandeling. Herseninfarct behandelingen zijn namelijk niet zonder risico, deze ratio wordt dus gebruikt om in te schatten of de risico's van de behandeling minder zwaar wegen dan het potentiele effect van de behandeling. Als laatste wordt een CTA gemaakt; dit is ook een contrast scan, maar hier wordt er maar op één tijdstip een CT scan gemaakt. Deze scan wordt gebruikt om het vaatstelsel van de patiënt in kaart te brengen. Hieruit kan worden opgemaakt waar de verstopping van het bloedvat zit, maar mogelijk ook wat de oorzaak van verstopping is. 
Een recente ontwikkeling op het gebied van CT beeldvorming is duale-energie CT (DECT). Bij een DECT scan wordt er niet van één spectrum van röntgenfotonen, maar van twee spectra met verschillende gemiddelde energie (een hoog en laag energetische spectrum) een beeld opgenomen. Om dit verschil duidelijk te maken, kan je een conventionele CT scan zien als een zwart/wit foto, terwijl met DECT een kleurenfoto wordt gemaakt. Hierdoor verkrijg je meer informatie over het afgebeelde orgaan. Het wordt bijvoorbeeld mogelijk om virtueel beelden op verschillende energie niveaus te reconstrueren, maar wordt het ook mogelijk om materialen te onderscheiden en te kwantificeren. Laag energetische virtuele beelden zijn gevoelig voor jodiumhoudend contrast middel, terwijl hoog energetische het hersenweefsel met minder ruis afbeelden. Deze techniek heeft dus de potentie om een herseninfarct op de NCCT beter te visualiseren of om bij een contrastmiddel houdende scan zoals de CTP scan, de CTP analyse te verbeteren.

Er zijn op dit moment een aantal klinisch beschikbare methodes om deze twee verschillende spectra op te nemen; snel schakelen tussen twee energieën ( $k V$ switchen), het gebruik van twee röntgenbuizen of een dubbellaags detector dat 1 spectrum kan scheiden in 2 spectra.

Naast de genoemde voordelen heeft CT ook een belangrijk nadeel. Een CT scanner maakt gebruik van röntgenstraling. Blootstelling aan röntgenstraling verhoogt het risico op kanker, en over het algemeen geldt; hoe meer straling, hoe hoger dit risico. Om deze risico's zo laag mogelijk te houden is er het "as low a reasonably achievable" (ALARA) principe. Volgens het ALARA principe moet elke CT scan met een zo laag als redelijkerwijs mogelijke stralingsdosis gemaakt worden. Dit betekent dat vooral bij de CTP scan, maar ook bij de NCCT veel ruis aanwezig is wat in verschillende mate effect heeft op de diagnostische kwaliteit van de scans.

\section{Proefschrift}

Dit proefschrift had als doel de mogelijkheden van hoge-resolutie CT perfusie en duale-energie CT voor het CT scan protocol voor acute beroerte te evalueren. We hebben vooral gekeken naar het verbeteren van de diagnostische kwaliteit voor herseninfarctdetectie door gebruik te maken van DECT, maar ook naar het gebruik van DECT bij CT perfusie. Daarnaast werd er ook gekeken of hoge-resolutie CTP de detectie van klein volume infarcten kan verbeteren.

Dit proefschrift bestaat uit 3 delen; in deel 1 is er vooral gefocust op de technische aspecten van DECT en is dit vergeleken met conventionele CT. In Hoofdstuk 2 werd de stralingsdosis die hoort bij een dubbellaags detector CT scanner (DLCT) bekeken en hoe deze zich verhoudt tot de stralingsdosis van een conventionele enkellaags detector CT scanner (SLCT). 


\section{Appendices}

De introductie van een dubbellaags detector zou namelijk een verhoging in de dosis kunnen betekenen ten opzichte van een enkellaags detector CT scanner. Wat we zagen was dat het gebruik van een DLCT geen verhoging liet zien van de stralingsdosis ten opzichte van de SLCT, maar dus wel de extra informatie bood van een duale-energie CT scanner. In Hoofdstuk $\mathbf{3}$ is de beeldkwaliteit van conventionele CT beelden van de DLCT vergeleken met die van de SLCT met behulp van fantoom scans. Net als met de dosis, kan de beeldkwaliteit ook beïnvloedt kunnen worden met de introductie van een dubbellaags detector. We vonden dat de beeldkwaliteit van de DLCT vergelijkbaar is met de SLCT bij middelmatig grote fantomen, en dat de beeldkwaliteit iets verminderde bij fantomen van meer dan 30 centimeter in diameter. Dit betekent dat de beeldkwaliteit van de CT scans van een patiënt met een gemiddeld formaat niet verminderd zal zijn, maar dat grote patiënten een iets verminderde beeldkwaliteit zullen hebben. Vervolgens hebben we gekeken naar de beeldkwaliteit van het hersenweefsel in virtueel monochromatische CT beelden zonder contrast in vergelijking met conventionele $\mathrm{CT}$ in Hoofdstuk 4. Wat we vonden was dat de optimale beeldkwaliteit van het hersenweefsel met virtueel monochromatische beelden wordt verbeterd ten opzichte van conventionele CT, maar ook dat de beeldkwaliteit sterk afhankelijk was van de gebruikte reconstructie kernel en afhankelijk was van de indicatie om de NCCT uit te voeren.

Deel 2 van het proefschrift ging over de toegevoegde waarde van duale-energie CT bij de diagnose van herseninfarcten. In Hoofdstuk $\mathbf{5}$ is er met een observerstudie gekeken naar de virtueel monochromatische beelden van de NCCT voor de detectie van herseninfarcten. In hoofdstuk 4, hebben we namelijk laten zien dat monochromatische beelden een verbeterd contrast-ruis verhouding hebben en beeld artefacten verminderen en dus misschien voor een verbeterde infarct detectie kunnen zorgen. Uit deze studie concludeerden we dat de virtueel monochromatische NCCT beelden bij 80 - 90 keV de hoogste nauwkeurigheid hebben voor de detectie en locatiebepaling van herseninfarcten. In Hoofdstuk $\mathbf{6}$ is er gekeken naar een andere van de dualeenergie NCCT afgeleide reconstructie: de virtuele herseninfarct scan. Dit type scan zorgt voor een verminderde grijs-witte stof differentiatie, maar makkt geinfarceerd weefsel een stuk duidelijker. Wat we vonden was dat met behulp van de virtuele herseninfarct scan de infarct-kern volumes en de herseninfarct locaties significant nauwkeuriger bepaald konden worden dan met conventionele CT. In Hoofdstuk 7 hebben we een observerstudie opgezet waarbij we de kwaliteit van perfusieparameter-afbeeldingen van virtueel monochromatische CT perfusie scans gemaakt met een dubbellaags detector CT scanner evalueerden en vergeleken met de huidige klinische standaard. Laag energetisch monochromatische beelden liggen dichterbij de zogenoemde "k-edge" van jodium en zouden dus mogelijk voor een verhoogd contrast in CTP kunnen zorgen. 
De beelden bevatten echter ook een stuk meer ruis dan conventionele CT beelden. We hebben bewezen dat de beeld- en visuele-kwaliteit van 50 keV CT perfusie scans een significante verbetering ten opzichte van de conventionele huidige klinische standaard opleverde.

Deel 3 van het proefschrift ging over de technische aspecten van CT perfusie bij de beeldvorming van herseninfarcten. In Hoofdstuk $\mathbf{8}$ keken we naar het effect van verschillende acquisitie-intervallen op de perfusieparameters en de infarct-volumes bij drie verschillende CT perfusie analyse methodes. Kortere acquisitie intervallen zorgen voor een nauwkeurigere monitoring van de contrastvloeistof, maar het is soms om technische redenen niet mogelijk om met korte intervallen te scannen en dan zijn er dus langere acquisitie intervallen nodig. We vonden dat aanpassen van de acquisitie intervallen kan resulteren in een effect op de perfusieparameters, maar dat we voor dit effect kunnen compenseren door de perfusie afbeeldingen te kalibreren. Hiermee blijft het mogelijk om gezond weefsel van infarctweefsel te onderscheiden. Daarnaast werd het infarct-volume wel beïnvloedt door het acquisitie interval, maar visuele inspectie van de volumes liet zien dat de infarct summary map weinig verschillen liet zien tussen acquisitie intervallen met 1 seconden tot en met 4 of 5 seconden. Als laatste, is er in Hoofdstuk 9 gekeken naar dunne coupe CT perfusie scans om de detectie van kleine herseninfarcten te evalueren. Het is namelijk gesuggereerd dat met dunne coupe CTP de detecteerbaarheid van kleine herseninfarcten verbeterd kan worden. Dunne coupe CTP wordt alleen gekenmerkt door hoge ruis niveaus en vereisen dus geavanceerde filters om de ruis te onderdrukken. Hiervoor hebben we twee bilateraal filters vergeleken: het TIPS bilateraal filter en een bilateraal filter begeleid door een afbeelding gecreëerd met hoofdcomponentenanalyse (PCA). We hebben laten zien dat met begeleid bilateraal filteren perfusie waardes nauwkeurig werden bepaald, met een hoog contrast. Al blijft het detecteren van kleine infarcten lastig, klein volume infarcten werden met hogere sensitiviteit gedetecteerd en met significant verbeterde diagnostische zekerheid en beeld kwaliteit met het begeleid bilateraal filter in vergelijking met het TIPS filter.

Concluderend hebben we in dit proefschrift de potentiële meerwaarde van dualeenergie CT aangetoond bij de visualisatie en detectie van herseninfarcten met zowel CT zonder contrast als bij CT perfusie. Dit kan worden bereikt zonder verhoging van de stralingsdosis, maar ook zonder verlies van beeldkwaliteit in vergelijking met conventionele CT. Daarnaast hebben we laten zien dat wanneer het axiale CT perfusie bereik vergroot moet worden en daarmee het acquisitie interval verlengt, dit nog steeds nauwkeurige en betrouwbare $\mathrm{CT}$ perfusie resultaten geeft. Bovendien is het met begeleide bilateraal filter methodes mogelijk om de detecteerbaarheid van klein volume infarcten te verbeteren. 


\section{Appendices}

\section{Acknowledgments}

En dan zijn we nu aangekomen bij het belangrijkste onderdeel van een proefschrift; het dankwoord. Ik denk dat er door de jaren heen een flink aantal mensen in meer of mindere mate hebben bijgedragen aan de totstandkoming van dit proefschrift: dank daarvoor. Ik wil er graag een aantal in het speciaal bedanken.

Allereerst wil ik mijn ouders bedanken voor hun onvoorwaardelijke steun tijdens al die jaren op school en tijdens de 4 jaar van mijn promotie. We hebben als gezin de laatste jaren flink wat meegemaakt, en daarom is dit proefschrift ook voor een groot gedeelte van en voor jullie! Ik hou van jullie. Mijn paranimfen en tevens mijn broertjes, Jelmer en Trystan. Ik vind het heel bijzonder dat we samen als broers de verdediging van dit proefschrift mogen meemaken. Ik ben een trotse (semi) grote broer en ik had dit nooit zonder jullie gekund.

Dan wil ik natuurlijk graag mijn promotie team bedanken. Hugo, om te beginnen, heel erg bedankt dat je mij de kans hebt gegeven om te promoveren, en het me vervolgens ook nog eens hebt laten afmaken. Ik heb veel van je geleerd, en heb heel erg prettig met je samengewerkt. De jaarlijkse barbecue en het groepsweekend waren heel erg leuk en gaan zeker gemist worden. Max, ook al ging je met pensioen toen ik nog niet zo heel lang bezig was, ik heb altijd het gevoel gehad dat mijn werk met veel zorg en nauwkeurigheid werd bekeken. Je input werd dan ook altijd zeer gewaardeerd. Daarnaast waren de gesprekken die we voerden ook altijd erg gezellig.

Mijn copromotoren Edwin en Jan Willem. Edwin, wat heb jij een engelen geduld. Ik denk dat ik de afgelopen 4 jaar zeker 10000 vragen aan je hebt gesteld, en je hebt ze allemaal met dezelfde duidelijkheid en hetzelfde enthousiasme beantwoord. Jou hulp, enthousiasme en je eigen proefschrift (lees: CT perfusie bijbel) hebben er zeker voor gezorgd dat mijn promotie heel erg soepel is verlopen: bedankt daarvoor. Jan Willem, door de jaren heen ben ik er achter gekomen dat je van lego houdt, een uitstekende muzieksmaak hebt, maar bovenal een scherpe en begenadigd wetenschapper bent. Ik kon altijd bij je terecht met alles wat er op dat moment speelde, en daar ben ik je erg Dankbaar voor (en ja, dat is een verwijzing naar je achternaam (:)).

De rest van de ENCLOSE groep: Frans jij hielp me altijd uit de brand als er weer eens wat ingetekend moest worden of als ik wat klinische gegevens nodig had. Daarnaast hebben we hele leuke tijden gehad in Stanford, Wenen en natuurlijk in Utrecht. Het was fijn met je samenwerken en ik denk dat ze er straks een hele goede neuroloog bij hebben. Birgitta; ik was niet een "officiële" promovendus van jou, maar zo voelde het vaak wel. Je had altijd goede en bruikbare input tijdens de meetings en de etentjes in Wenen ga ik echt nooit meer vergeten. 
Richard, ook al ben je in opleiding tot radioloog en zagen we je dus niet zo heel veel. De keren dat je er was, was je ook echt aanwezig. Ik denk dat jou lach 1 van de meest aanstekelijke is die ik ooit gehoord hebt. Daarnaast heb je ook nog de juiste humor: echt de perfecte combinatie. Als laatste Anneloes, ik denk dat ENCLOSE zonder jou hulp volledig was vast gelopen. Bedankt voor je inzet en hulp met de inclusies!

Leden van de beoordelingscommissie, prof. dr. G.J. Biessels, prof. dr. J. Hendrikse, prof. dr. R.M. Dijkhuizen, prof. dr. T. Leiner en prof. dr. C.B.L.M. Majoie, hartelijk dank voor het beoordelen van mijn proefschrift.

Mijn kamergenoten; Sandra wat kon jij die telefoon toch goed opnemen, dat hebben we de afgelopen jaren echt wel gemist. Daarnaast was jij ook altijd vroeg op werk, dus konden we altijd even een theetje drinken voor de drukte. En met de drukte bedoel ik jou Remco, man-man-man, wat kon jij slap lullen, maar wat heb ik er van genoten. Ik heb het de afgelopen tijd nog gemist ook! Ik hoop dat je het naar je zin hebt in Amerika, en ik kom zeker nog een keer langs om te zien wat dat Johns Hopkins nou allemaal wel niet voorstelt (bij dezen heb ik mezelf uitgenodigd). Martijn, de stille genieter en harde werker van onze kamer, je bent door je promotie heen gevlogen en tot de dag van vandaag weet ik nog steeds niet of het nou goed was als de lijntjes naast, boven of door elkaar heen liepen. Het blijft wel 1 van mijn grootste falen bij de klinisch fysica dat ik jou nooit aan de thee gekregen heb. Ik weet zeker dat het me ooit nog gaat lukken. Britt, zonder jou hadden onze planten het nooit overleefd, of naja minder lang overleefd. Ik vond het leuk om met een oud MNW-er op de kamer te zitten en ik weet zeker dat ik jou nog vaak ga tegen komen bij de klinische fysica. Als laatste Daan, eindelijk was daar iemand die ook iets met CT perfusie deed. De laatste tijd zaten we veelal met elkaar op de kamer en ik ben er achter dat jij (net als ik) wel van klagen houdt, dus dat waren altijd mooie gesprekken. Je droge humor was daar ook een welkome gast bij! Ik weet zeker dat jij een hele mooie promotie tegemoet gaat.

Dan de heren en dame van de klinische fysica; Arnold bedankt dat ik altijd voor alle $\mathrm{CT}$ gerelateerde vragen en problemen bij jou terecht kon. Bastiaan bedankt voor alle scouting tips en het organiseren van het jaarlijkse groepsweekend. Rob, topgozer, ik heb me zwaar vermaakt in de Tivoli en op Valhalla; moeten we zeker nog een keer overdoen. Dan Casper, wat ben jij een prachtvent. Ik heb nog nooit iemand zo goed zien dansen na een paar biertjes. Ik denk alleen dat je voorzichtiger moet zijn als je naar huis loopt. Bas en Marjolein, ook al hebben we elkaar amper gezien, jullie zijn een goede en leuke toevoeging voor de klinisch fysica groep. 


\section{Appendices}

Pascal en Frank, ik was blij dat er eindelijk mensen bij de groep kwamen die ook met enige regelmaat voetbal keken; eindelijk wat kwalitatief hoogstaande gesprekken! Martina, Achmed, Sander, Woutjan, Maryam, Leo, en Wilco bedankt voor de leuke en gezellige lunch en koffie gesprekken; het was een welkome afleiding.

Dan wil ik iedereen bedanken met wie ik over de afgelopen jaren op congres ben geweest: Mimmount, Bianca, Niels, Robbert, Carlo en Bas. Het was erg gezellig! Bas ik wil jou nog even in het speciaal bedanken, de RSNA was echt geniaal en de ISI borrels/etentjes waren zeker de moeite waard door jouw humor! Vervolgens wil ik iedereen bij ISI en de radiologie afdeling bedanken voor alle hulp, gezelligheid en leuke uitjes. Een speciaal bedankje voor Tineke van het technisch cluster: je staat altijd voor iedereen klaar en het feit dat je een aantal keer thee voor ons bestelde werd heel erg gewaardeerd $(-)$.

Next, I would like to thank Max for allowing me to come to Stanford. I learned a lot, and I want to thank you for always being as positive and supportive of everything I tried during my six month stay as you have been. Susan, without you I would not have been able to come to Stanford. Thank you for all your assistance during the application for visas, forms and all other paperwork. Jeremy, Guangming and Dylan you guys have been a great help in getting the research from Stanford published: thank you for all your help. To the other members of the neuroradiology group; Bin, Hui and Haijun, thank you for welcoming me with open arms. I enjoyed every moment of it. Lastly, I would like to say to Martin (\& Blaire), Martin (Koci), Vera, Valery (\& Luca), Dominco (\& Robyn), Virginia, Ayman (\& Nour), Dominika and Gijs thank you all for making my stay at Stanford as fun and productive as it has been. I miss you guys!

A special thank you to Jennifer, Jim, Denise and Reid Kleckner for allowing me to stay at the cottage, and for all those lovely dinners. I always felt very welcome and enjoyed every moment at the cottage, thank you!

Matthijs, Job, Floris, Douwe, Carmen, Michelle en Diederik; doordat we eigenlijk elke week wel chillen, naar een feestje gingen of naar een wedstrijd van ons aller Ajax keken ben ik door mijn promotie heen gevlogen. Deze avonden gaven mij de nodige afleiding en zorgde ervoor dat ik elke dag weer voor de volle 100\% aan mijn promotie kon werken. Bedankt dat jullie er zijn tijdens de leuke momenten, maar vooral ook tijdens de wat minder leuke momenten. Ik hoop dat we dit met $z$ 'n alle nog vele jaren vol kunnen houden!

Ultima pero no menos importante, quiero agradecer a Kassandra. Conocerte fue uno de las mejores momentos de ir a Stanford. Espero con mucha emoción nuestro futuro juntos. Te amo. 
Acknowledgments | B 


\section{Appendices}

\section{List of Publications}

\section{Publications}

GD van Praagh, NR van der Werf, J Wang, F van Ommen, D Fleischmann, MJW Greuter, T Leiner and MJ Willemink. Fully Automatic Quantification Method (FQM) of Coronary Calcium in an Anthropomorphic Phantom. Submitted.

DN Wolman, F van Ommen, E Tong, F Kauw, JW Dankbaar, E Bennink, HWAM de Jong, L Molvin, M Wintermark and JJ Heit. Dual-energy CT virtual ischemia maps improve estimation of core infarct size. Submitted.

F Kauw, F van Ommen, E Bennink, MJ Cramer, LJ Kappelle, RAP Takx, BK Velthuis, MA Viergever, HW van Es, WJ Schonewille, JM Coutinho, CBLM Majoie, H Marquering, HWAM de Jong and JW Dankbaar (2020). Early detection of small volume stroke and thromboembolic sources with computed tomography: Rationale and design of the ENCLOSE study. European Stroke Journal, in press.

F Kauw, JW Dankbaar, MW Blake, VY Ding, DB Boothroyd, F van Ommen, HWAM de Jong, LJ Kappelle, BK Velthuis, JJ Heit and M Wintermark (2020). Collateral status in ischemic stroke: a comparison of CT angiography, CT perfusion and digital subtraction angiography. Journal of Computer Assisted Tomography, in press.

F van Ommen, JW Dankbaar, G Zhu, DN Wolman, JJ Heit, F Kauw, E Bennink, HWAM de Jong and M Wintermark (2020). Virtual monochromatic dual-energy CT reconstructions improve detection of cerebral infarct in patients with suspicion of stroke. Neuroradiology, in press.

F van Ommen, F Kauw, E Bennink, JJ Heit, DN Wolman, JW Dankbaar, HWAM de Jong and M Wintermark (2020). Image quality of virtual monochromatic reconstructions of non-contrast CT on a dual-source CT scanner in adult patients. Academic Radiology, in press.

F van Ommen, E Bennink, JW Dankbaar, F Kauw and HWAM de Jong (2020). Improving the quality of cerebral perfusion maps with monoenergetic dual-energy computed tomography reconstructions. Journal of Computer Assisted Tomography, in press.

F Kauw, JJ Heit, BW Martin, F van Ommen, LJ Kappelle, BK Velthuis, HWAM de Jong, JW Dankbaar and M Wintermark (2020). Computed tomography perfusion data for acute ischemic stroke evaluation using rapid software: pitfalls of automated postprocessing. Journal of Computer Assisted Tomography, 44:75-77. 
F van Ommen, F Kauw, E Bennink, JW Dankbaar, MA Viergever and HWAM de Jong (2019). Effect of prolonged acquisition intervals for CT-perfusion analysis methods in patients with ischemic stroke. Medical Physics, 46:3156-3164.

F van Ommen, HWAM de Jong, JW Dankbaar, E Bennink, T Leiner and AMR Schilham (2019). Dose of CT protocols acquired in clinical routine using a dual-layer detector CT scanner: a preliminary report. European Journal of Radiology, 112:65-71.

F van Ommen, E Bennink, A Vlassenbroek, JW Dankbaar, AMR Schilham, MA Viergever and HWAM de Jong (2018). Image quality of conventional images of dual-layer SPECTRAL CT: a phantom study. Medical Physics, 45:3031-3042.

G Schooneveldt, HP Kok, E Balidemaj, ED Geijsen, F van Ommen, J Sijbrands, A Bakker, JJMCH de la Rosette, MCCM Hulshof, TM de Reijke and J Crezee (2016). Improving hyperthermia treatment planning for the pelvis by accurate fluid modeling. Medical Physics, 43:5442-5452.

\section{Conference proceedings}

F van Ommen, F Kauw, E Bennink, JW Dankbaar and HWAM de Jong. Effect of prolonged acquisition intervals on CT brain perfusion analyses. European Congress of Radiology, Vienna, 2019 (Oral presentation).

F van Ommen, HWAM de Jong, T Leiner, JW Dankbaar, E Bennink and A Schilham. Dose of CT protocols acquired in clinical routine using a dual-layer detector CT scanner. Radiological Society of North America Annual Meeting, Chicago, 2018 (Poster presentation).

F van Ommen, E Bennink, A Vlassenbroek, JW Dankbaar, AMR Schilham, MA Viergever and HWAM de Jong. Image quality of conventional images of dual-layer SPECTRAL CT: a phantom study. European Congress of Radiology, Vienna, 2018 (Oral presentation). 


\section{About the Author}

Fasco van Ommen was born on the $18^{\text {th }}$ of July 1992 in Hilversum. He went to secondary school at Laar \& Berg in Laren (NH), where he graduated the International Baccalaureate Middle Year's Program in 2008, and graduated VWO in 2011. In 2011, he started his bachelor's degree in Medische Natuurwetenschappen at the Vrije Universiteit in Amsterdam. He obtained his Bachelor of Science degree in 2014, and continued with a master degree in Medical Natural Sciences with specialization Medical Physics at the Vrije Universiteit. During this time, he gained research experience in the Department of Radiology and Nuclear Medicine and the Department of Radiation Oncology at the VU medical center (Amsterdam UMC-VUmc). He obtained his Master of Science degree in 2016. Fasco started his PhD at the University Medical Center Utrecht in 2016 under the supervision of prof. Hugo de Jong, prof. Max Viergever, dr. Edwin Bennink and dr. Jan Willem Dankbaar. In 2019, he went to the Stanford University Medical Center for an internship under the supervision of prof. Max Wintermark. The results of this work are described in this thesis. 



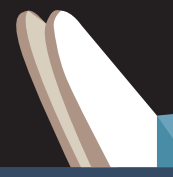

